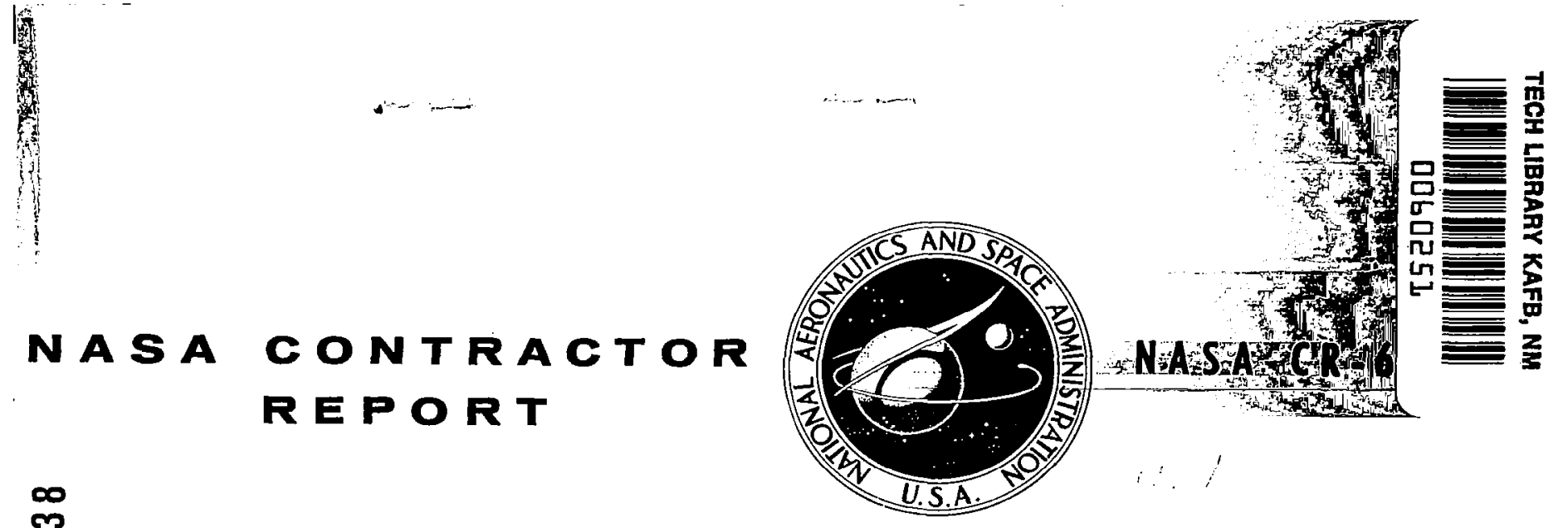

LOAN COPY: RETURN W AFWL (WLIL-2) KIRTLAND AFB, N NEX

\title{
RESEARCH ON REFURBISHABLE
} THERMOSTRUCTURAL PANELS FOR MANNED LIFTING ENTRY VEHICLES

by A. H. LaPorte

Prepared by

MARTIN COMPANY

Baltimore, Md.

for Langley Researcb Center

ATIONAL AERONAUTICS AND SPACE ADMINISTRATION - WASHINGTON, D. C. - NOVEMBER 1966 
NASA CR-638

RESEARCH ON REFURBISHABLE THERMOSTRUCTURAL PANELS FOR MANNED LIFTING ENTRY VEHICLES

By A. H. LaPorte

Distribution of this report is provided in the interest of information exchange. Responsibility for the contents resides in the author or organization that prepared it.

Prepared under Contract No. NAS $1-5253$ by MARTIN COMPANY Baltimore, Md.

for Langley Research Center

\section{NATIONAL AERONAUTICS AND SPACE ADMINISTRATION}

For sale by the Clearinghouse for Federal Scientific and Technical Information Springfield, Virginia 22151 - Price $\$ 3.75$ 


\section{TABLE OF CONTENTS}

Page

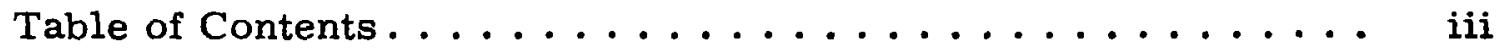

List of Illustrations ......................... . . . . .

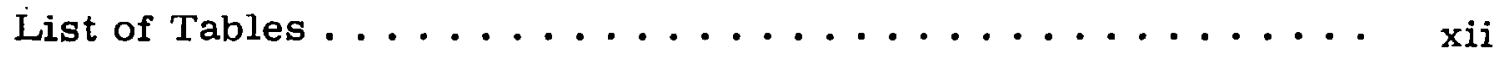

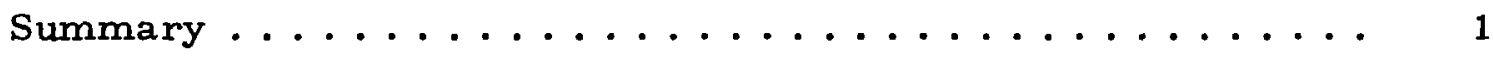

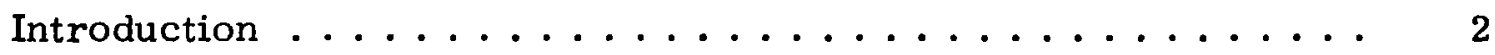

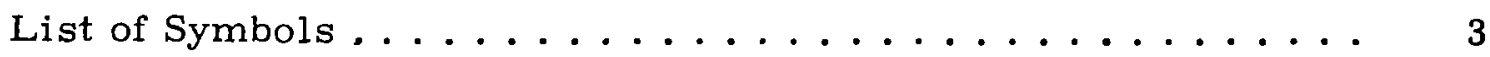

Preliminary Substrate Concept and Material Selection . . . . . 4

Discussion of Candidate Concepts and Materials ........ 4

Analysis of Candidate Thermostructural Panel Concepts . . . 14

Manufacturing and Quality Control Review of Panel

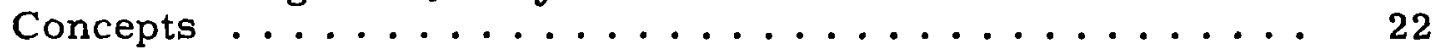

Material and Fabrication Cost Comparisons .......... 29

Concepts Comparison Chart................. 32

Selection of Thermostructural Panel Concepts ......... 33

Analysis and Optimization of Selected Thermostructural

Panel Concepts ...................... 36

Panel Size and Orientation Studies $\ldots \ldots \ldots . \ldots . \ldots 36$

Selection of Heat Shield Panel Sizes and Orientation . . . . 38

Joint Design Considerations . . . . . . . . . . . . . 40

Substrate Panel/Support Interaction and Flexibility

Requirements ..................... 45

Heat Shield Sizing and Thermal Analysis . . . . . . . . 47

Substrate Panel Optimization and Structural Analysis . . . . 57

Application of Design Data................ 63

Fabrication and Cost Data Comparisons. . . . . . . . 72 


\section{TABLE OF CONTENTS (continued)}

Page

Vehicle Heat Shield Weight Comparisons .......... 75

Selection of Optimum Thermostructural Panel Concepts . . . 76

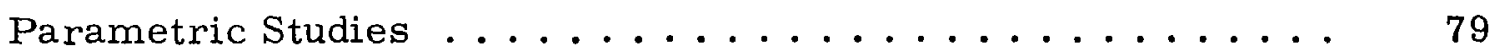

Variation of Environmental Factors ............ 79

Variation of Material Ablation and Heat Transfer

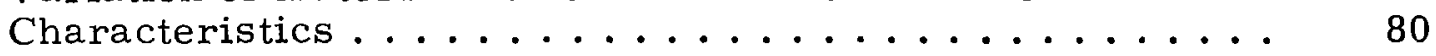

Variation of Ablator Allowable Strain ............ 80

Weight Comparisons of Various Lifting Entry Vehicle

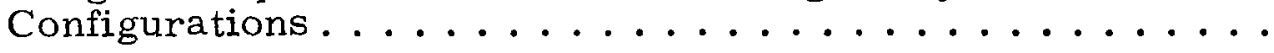

Design Development Problem Areas and Concept

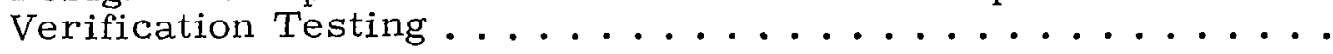

Design Development Problem Areas ............ 81

Concept Verification Testing ............... 84

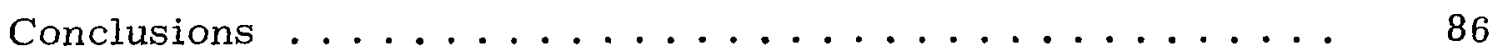

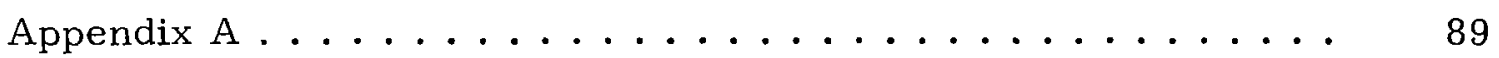

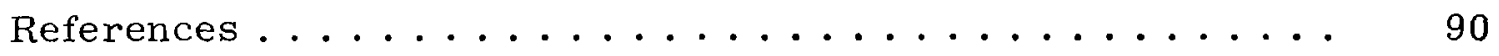




\section{LIST OF FIGURES}

Figure

Title

Page

1 Double Wall Ablative Heat Shield Concept . . . . .

2 Preliminary Substrate Panel Concepts ........

3 Candidate Metal Substrate Panel Materials

(E/ $\rho$ Versus Temperature)..............

4 Candidate Metal Substrate Panel Materials

$\left(\sigma_{\text {ult }} / \rho\right.$ Versus Temperature $)$........... 95

5 Candidate Metal Substrate Panel Materials

$\left(\sigma_{\mathrm{y}} / \rho\right.$ Versus Temperature $) \ldots . . . \ldots 96$

6 Candidate Plastic Laminate Substrate Panel

Materials (E Versus Temperature) .........

7 Candidate Plastic Laminate Substrate Panel

Materials ( $\sigma_{\text {ult }}$ Versus Temperature) ...... 98

8 Candidate Adhesive Bond Materials (Tensile

Shear Strength Versus Temperature)........

9 Candidate Insulation Materials ( $\rho \mathrm{k}$ Versus

Temperature) ..................... 100

10 Candidate Insulation Materials (Thermal

Diffusivity Versus Temperature)...........

11 Multilayer Microquartz Specific Heat Versus

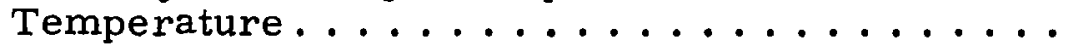

12 Multilayer Microquartz Thermal Conductivity

Versus Temperature at $8 \mathrm{~mm} \mathrm{Hg} \mathrm{.........}$

13 Low Density Nylon Phenolic Specific Heat for

Virgin and Char Material .............. 104

14 Low Density Nylon Phenolic Thermal

Conductivity for Virgin Material. . . . . . . . . 104

15 Low Density Nylon Phenolic Char Thermal

Conductivity ..................... 105

16 Instantaneous Coefficient of Thermal

Expansion for Low Density Nylon Phenolic . . . . 106 


\section{LIST OF FIGURES (continued)}

Figure

Title

Page

17 Low Density Nylon Phenolic Ultimate Strain Data. . . 107

18 NASA 602 Specific Heat for Virgin and Char

Material.................... 108

NASA 602 Thermal Conductivity ........... 109

20 NASA 602 Instantaneous Coefficient of Thermal

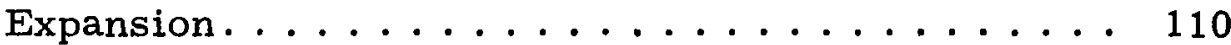

21 NASA 602 in Honeycomb Matrix Ultimate Tensile

Strain Versus Temperature .............. 111

22 Phenolic/Glass Laminate Thermal Conductivity. . . 112

23 Idealized Re-entry Heating Pulse .......... 113

24 Ablator Weight Versus Maximum Bondline

Temperature.................. 114

25 Thermal Stress Comparisons (uniform cold soak at $-110^{\circ}$ F or $\left.194.4^{\circ} \mathrm{K}\right) \ldots \ldots 115$

26 Honeycomb Substrate Panel Optimization

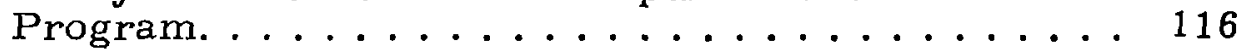

27 Single Skin Corrugation Substrate Panel

Optimization Program. ............. 117

28 Stainless Steel Honeycomb Panel Optimum

Weight Curves................. 118

29 Titanium Honeycomb Panel Optimum Weight

Curves.................. 119

30 Beryllium Honeycomb Panel Optimum Weight

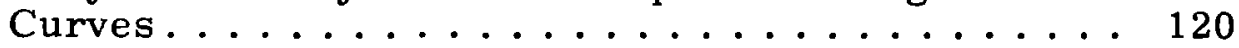

31 Phenolic/Glass Laminate Honeycomb Panel

Optimum Weight Curves ............. 121

32 Stainless Steel Single Skin Corrugation Panel

Optimum Weight Curves .............. 


\section{LIST OF FIGURES (continued)}

Figure

Title

Page

33 Titanium Single Skin Corrugation Panel Optimum

Weight Curves . . . . . . . . . . . . . . . 122

34 Beryllium Single Skin Corrugation Panel

Optimum Weight Curves ............... 123

35 Phenolic/Glass Laminate Single Skin Corrugation

Panel Optimum Weight Curves ............ 123

36 Stainless Steel Double Skin Corrugation Panel

Optimum Weight Curves ............... 124

$37 \quad$ Titanium Double Skin Corrugation Panel

Optimum Weight Curves .............. 125

38 Beryllium Double Skin Corrugation Panel

Optimum Weight Curves ............. 126

39 Phenolic/Glass Laminate Double Skin Corruga-

tion Panel Optimum Weight Curves . . . . . . . 127

40 Optimum Weight Curves for Honeycomb Panels

at Room Temperature... . . . . . . . . . . 128

41 Optimum Weight Curves for Single Skin

Corrugation Panels at Room Temperature . . . . . . 129

42 Optimum Weight Curves for Double Skin Corrugation Panels at Room Temperature ......... 130

43 Flexible Support Strength/Panel Size Interaction for a Steel Panel at $800^{\circ} \mathrm{F}\left(700^{\circ} \mathrm{K}\right)$ and Phenolic/Glass Support.............. 131

44 Refurbishable Ablator Heat Shield Panel Orientation (Top View) . . . . . . . . . . . . . 132

45 Refurbishable Ablator Heat Shield Panel

Orientation (Bottom View) . . . . . . . . . . . 133

46 RTV 560 Silicon Rubber Low Temperature

Ultimate Strain Data. . . . . . . . . . . . . 134 


\section{LIST OF FIGURES (continued)}

Figure

Title

Page

47 Thermal Stresses in Rigidly Supported Plastic

Laminate Substrate Panels ............ 135

48 Thermal Stresses in Rigidly Supported Stain-

less Steel Substrate Panels ............ 136

49 Heat Shield Design Curve (Nominal Trajectory,

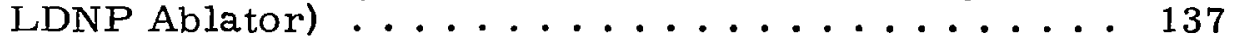

50 Heat Shield Design Curve (Nominal Trajectory,

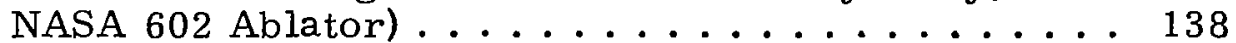

51 Heat Shield Design Curve (Overshoot Trajectory,

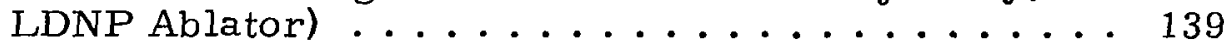

52 Heat Shield Design Curve (Overshoot Trajectory,

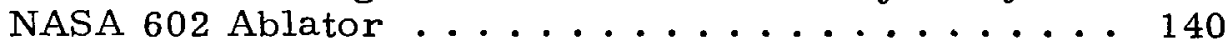

53 Comparison of Ablator Thickness Design Curves

on a Total Heat Basis ............... 141

54 Heat Shield Weight Curve (Nominal Re-entry

Trajectory).................. 142

55 Heat Shield Weight Curve (Overshoot Re-entry

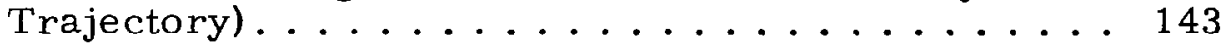

$56 \quad$ Critical Trajectories for Ablator Thickness

Determination (Low Density Nylon Phenolic) . . . . . 144

57 Critical Trajectories for Ablator Thickness

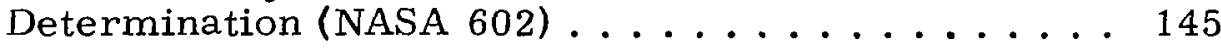

58 Comparison of Microquartz Thermal

Conductivity Values ................. 146

59 Pressure Dependence of Thermal Conductivity

of Microquartz/ Foil Insulation at Two Tem-

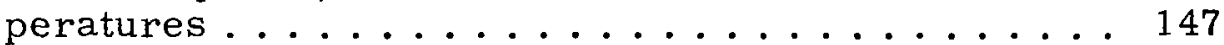

60 Integral Insulation/Support Concept Test

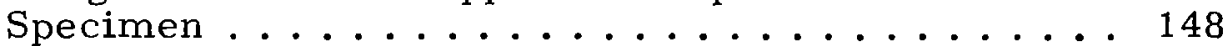




\section{LIST OF FIGURES (continued)}

Figure

Title

Page

61 Flexcore Honeycomb/Aluminum Foil Insulation

Optimization .................

62 Effective Thermal Conductivity Versus Temperature and Pressure for Flexcore Honeycomb with Aluminum Foil . . . . . . . . . . . . .

63 Pressure Dependence of Thermal Conductivity of Flexcore Honeycomb with Foil Insulation at Two

Temperatures ................

64 Insulation Conductivity/Thickness/Structure Temperature Interaction ............

Heat Short Problem Analytical Model .......

66 Maximum Structure Temperature at Touchdown with Heat Short Effect Considered . . . . . . . .

Effect of Support Spacing on Heat Short Effect. . .

68-81 Substrate Panel Design Constraints . . . . . . 156-169

82-95 Substrate Panel Design Optimization Curves . . . 170-183

96 Design Constraint Relationships for 17-7 PH

Steel Substrate with $1.50-$ In. $(3.81 \mathrm{~cm})$ NASA

602 Ablator, $70^{\circ} \mathrm{F}\left(294.4^{\circ} \mathrm{K}\right)$ Condition .......

97 Design Constraint Relationships for Phenolic/ Glass Substrate with 1:50-In. $(3.81 \mathrm{~cm})$ NASA 602 Ablator, $70^{\circ} \mathrm{F}\left(294.4^{\circ} \mathrm{K}\right)$ Condition .......

98 Design Constraint Relationships for Phenolic/ Glass Substrate with 1.50-In. $(3.81 \mathrm{~cm})$ LDNP Ablator, $70^{\circ} \mathrm{F}\left(294.4^{\circ} \mathrm{K}\right)$ Condition ..........

Optimum Design Moments for Substrate/Ablator Combinations, $70^{\circ} \mathrm{F}\left(294.4^{\circ} \mathrm{K}\right)$ Condition......

Panel Support Requirements for Optimum Moments, $70^{\circ} \mathrm{F}\left(294.4^{\circ} \mathrm{K}\right)$ Condition, 12-psi

$\left(62.74 \mathrm{kN} / \mathrm{m}^{2}\right)$ Pressure ............. 


\section{LIST OF FIGURES (continued)}

Figure

102 Critical Panel Moment Versus Support Span and Pressure (Aspect Ratio $=1.0$ ) .......

103 Critical Panel Moment Versus Support Span and Pressure (Aspect Ratio $=1.5$ ) .......

104 Attachment Weight Versus Load/Attachment (Rigid Support at Room Temperature) .......

Title

Page

190

191

192

Attachment Weight Versus Load/Attachment

(Flexible Support at Room Temperature) . . . . .

193

Flutter Boundary Criteria . . . . . . . . . . 194

HL-10 Flutter Envelope ............. 195

Curvature Correction Factor . . . . . . . . 196

Heat Shield Panel Locations. . . . . . . . . .

197

Heating Rate Ratio Envelopes for Panel Design

(Nominal Re-entry Trajectory) ..........

198

Heating Rate Ratio Envelopes for Panel Design

(Over shoot Re-entry Trajectory) .........

199

Leading Edge Panel Model for Structural Network Analysis . . . . . . . . . . . . . . . .

200

U1timate Panel Face Stresses (Leading Edge Substrate Panel, Maximum Load Factor Abort Con-

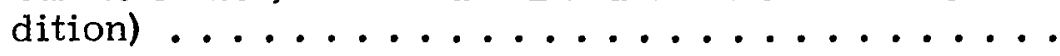

201

Critical Thermal Condition Stresses (Leading

Edge Substrate Panel)..............

202

Schematic Diagram of Fabrication Procedure,

Plastic Honeycomb Substrate (NASA 602 Ablator).

203

Schematic Diagram of Fabrication Procedure

(Plastic Honeycomb Substrate, LDNP Ablator) . .

204

Leading Edge Panel ..............

205 


\section{LIST OF FIGURES (continued)}

Figure

Title

Page

118

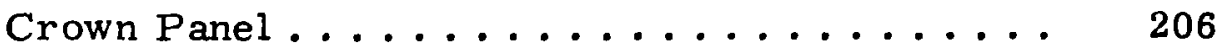

119

Alternate Designs for Attachment .......... 207

120

Effect of Variation in Heat Rate Factor on Heat

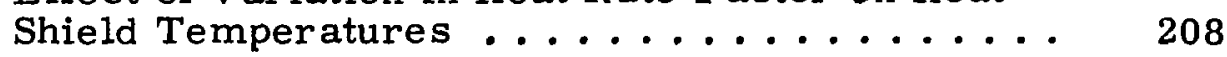

121 Effect of Variation in Heat Rate Factor on Heat

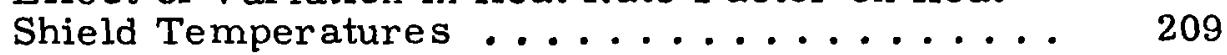

122 Effect of Variation in Vehicle W/S on Heat

Shield Temperatures ............... 210

123 Effect of Variation in Ablator Thermal Conductivity on Heat Shield Temperatures ...........

124 Effect of Variation in Ablator Thermal Conductivity on Heat Shield Temperature ...........

125 Effect of Variation in Ablator Density on Heat Shield Temperatures ..............

126 Effect of Variation in Ablator Density on Heat Shield Ternperatures ..............

127 Effect of Variation in Char Surface Recession Rates on Heat Shield Temperatures .........

128 Effect of Variation of Char Emissivity on Heat

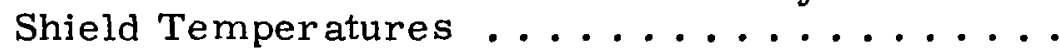

129 Effect of Variation of Transpiration Factor on Heat Shield Temperatures............ 


\section{LIST OF TAB LES}

Table

Title

Page

1 Preliminary Rating of Substrate Panel Concepts .....

2 Candidate Substrate Panel Materials . . . . . . . . .

3 Candidate Substrate Panel Materials after Preliminary Screening. . . . . . . . . . . . 9

4 Densities of Candidate Ablators . . . . . . . . . 12

5 Phenolic/Glass Laminate Thermal Properties ...... 14

6 Comparative Heat Shield Weights and Thicknesses.... 15

$7 \quad$ Material Properties for Cold Soak Thermal Stress Comparison ...................... 17

8 Substrate Panel Thickness Constraints for Thermal Stress Compatibility. . . . . . . . . . . . . 18

9 Minimum Gages for Candidate Substrate Pane1 Materials .....................

10 Substrate Panel Optimum Weight Comparisons at Room Temperature. . . . . . . . . . . . .

11 Summary of Fabrication Factors--Honeycomb

Substrate Panel ..................

$11 \mathrm{~b}$ Summary of Fabrication Factors--Skin-Corrugation Panels .......................

11c Summary of Fabrication Factors--Built-up Stiffened Skin Substrate Panel. . . . . . . . . . . 26

11d Summary of Fabrication Factors--Bead Stiffened 27

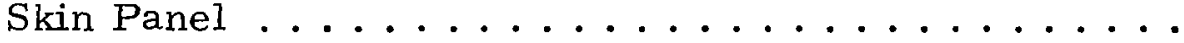

11e Summary of Fabrication Factors--Machined Waffle Skin Panel. ................ 28

12 Substrate Panel Material Cost Summary. . . . . . . . 30

13 Preliminary Cost Comparison Chart .......... 31 


\section{LIST OF TABLES (continued)}

Table

Title

Page

14 Concepts Comparison Chart. . . . . . . . . . . 34

15 Proposed Heat Shield Panel Breakdown . . . . . . . . 39

16 Joint Treatment Considerations . . . . . . . . . . 40

17 Minimum Joint Gap Sizes for a 4 x $6 \mathrm{ft}(1.219$

x $1.829 \mathrm{~m}$ ) (Orbital Cold Soak) . . . . . . . . . . 43

18 Joint Design Concepts . . . . . . . . . . . . . . . . 44

19 Effects of Support Spacing . . . . . . . . . . . 46

20 Substrate Panel/Vehicle Structure Interaction Loads

with a Rigid Support System . . . . . . . . . . . . 47

$21 \quad$ Critical $\dot{\mathrm{q}}_{\mathrm{L}} / \dot{\mathrm{q}}_{\mathrm{stag}}$ Ratios ................ 50

22 Comparison of Critical Heat Shield Temperatures for Undershoot Re-entry . . . . . . . . . . . . . 51

23 Comparison of Undershoot and Maximum Heating Rate Abort Trajectories ... . . . . . . . . . 52

24 Representative Optimum Moments for Panel Design

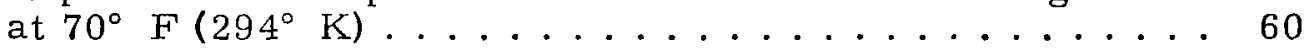

25 Comparison of Typical Panel Support Requirements

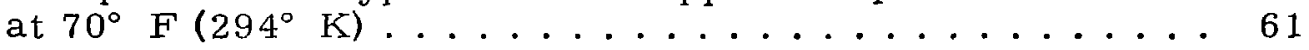

26 Critical Limit Design Pressures and Temperatures for Substrate Panel Design. . . . . . . . . . . 64

27 Ablator Thickness Ranges for Sample Panels .......66 65

28 Support Spacing Requirements for Various Panel Depths and Ablators . . . . . . . . . . . . 65

29 Comparative Support Distribution Densities . . . . . . 6 66

30 Attachment Ratio and Substrate Panel Weight Tradeoff . . 66

31 Comparison of Phenolic/Glass and Phenyl-Silane/ Glass Leading Edge Substrate Panels. . . . . . . . . . . 


\section{LIST OF TABLES (continued)}

Table

Title

Page

32 Comparison of Phenolic/Glass and Phenyl-Silane/

Glass Crown Substrate Panels . . . . . . . . . . . . 68

33 Cold Soak Temperature Distributions . . . . . . . . 71

34 Ablator Thermal Strain Comparisons ......... 71

35 Final Thermostructural Panel Cost Comparisons .... 74

36 Heat Shield System Weight Comparisons. . . . . . . 75

37 Comparison of Ablator Capabilities . . . . . . . . 76

38 Comparison of Substrate Panel Material Capabilities . . 78

39 Comparative Vehicle Heat Shield Weights........ 81

40 Element Test Breakdown for Composite Thermostructural Panel Design Verification .......... 85 


\title{
RESEARCH ON REFURBISHABLE THERMOSTRUCTURAL
}

\section{PANELS FOR MANNED LIFTING ENTRY VEHICLES}

\author{
By A. H. LaPorte
}

SUMMARY

Studies have been conducted on a group of candidate materials and structural concepts applicable to refurbishable composite ablative thermostructural heat shield panel configurations for manned lifting entry vehicles. Although some relatively advanced material concepts were briefly considered, only off-the-shelf materials with overall state-of-the-art capability were selected.

Practical design implications associated with the use of refurbishable heat shield panel concepts, such as panel size constraints, configuration of joints, substrate panel/support interactions, heat short effects associated with the panel support system, and fabrication and cost aspects, have been evaluated and integrated into the selection of a recommended thermostructural panel configuration.

Previously defined thermal and structural environmental parameters for a NASA Langley HL-10 lifting entry vehicle have been applied to the development of heat shield design curves and structural configurations. Critical design cases for thermal and structural analyses have been defined.

Structural optimization studies were performed on a group of substrate panel configurations in order to define optimum weights within a set of design constraints. Significant factors influencing the optimum substrate panel design are defined. The inter-relationship between required panel support spacing and ablator and substrate panel materials was evaluated and found to be significant. The panel flutter problem was examined and defined in terms of the HL-10 flight envelope parameters.

The concept of an integral insulation/support system was investigated and shown to provide potential design advantages with further development.

Total heat shield weights were calculated and compared for the two most promising systems on the HL-10 vehicle. Further comparisons were made of the total heat shield weights of the selected concept for the HL-10, M2-F2 and SV-5 configurations. 
The concept selected consisted of a NASA 602 elastomeric ablator supported by a honeycomb matrix which is bonded to a phenolic/glass laminate honeycomb substrate panel. The adhesive bond material is HT-424. The substrate panel is supported on the basic vehicle structure by a pattern of molded phenolic/glass laminate flanged cups. The gap between the substr ate panel and vehicle structure is filled with multilayer microquartz insulation material.

\section{INTRODUCTION}

In recent years, much study has been directed toward the lifting vehicle concept for manned near-earth missions such as logistic ferry applications. Economic and operational considerations indicate the desirability of designing a multimission capability into the se vehicles. Accordingly, either the vehicle components should be reusable with a minimum of refurbishment or, if economically feasible, they may be disposed of and replaced.

This study deals with a specific class of heat shield concepts that have been studied previously for application to multimission manned lifting entry vehicles (ref. 1). The double wall ablative heat shield (Figure 1) consists of an ablative material attached to a rigid substrate panel which in turn is supported on the basic vehicle structural shell by an attachment system. The space between the substrate panel and structural shell is filled with a low density insulation system. The basic program objective was to survey a broad spectrum of combinations of presently available material and structural components of a refurbishable composite thermostructural panel system. A preliminary screening study was performed in order to define the most promising concepts and materials. Subsequently, a detailed engineering analysis was performed on the selected configurations in order to develop comparative data as a basis for the final selection. Consideration was given, in the order of their importance, to the reliability, weight, and cost of each of the configurations.

This investigation was conducted under NASA-LRC Master Agreement Contract No. NAS 1-5253, Task Order Number 1, "Re search on Refurbishable Thermostructural Panels for Manned Lifting Entry Vehicles." The study was performed by the Martin Company, Baltimore Division. Dr. J. M. Hcdgcpcth was Program Manager and Mr. W. F. Barrett was the program liaison engineer. Mr. A. H. LaPorte was responsible for the technical direction of the program and was assisted by other members of the Martin Company engineering staff including Mr. R. E. Rieckmann and Mr. H. H. Hotchkiss. Other contributors 
to the program included Mr. J. Cincotta, F. Schor, B. Graham, F. Keefe, A. Ber'wizky, J. Bontya, F. Levinsky, and S. Kozlow.

Mr. C. M. Pittman of the Structures Research Division, Langley Research Center, Hampton, Virginia, was the technical representative for the project.

\section{LIST OF SYMBOLS}

\begin{tabular}{|c|c|}
\hline A & element cross-sectional area \\
\hline$a$ & lengthwise support spacing \\
\hline $\mathrm{b}$ & peripheral support spacing \\
\hline$d_{L}$ & longitudinal panel dimension \\
\hline $\mathrm{d}_{\mathrm{P}}$ & peripheral panel dimension \\
\hline$E$ & modulus of elasticity \\
\hline h & substrate panel depth \\
\hline $\mathrm{k}$ & thermal conductivity \\
\hline $\mathrm{M}$ & moment \\
\hline$M_{\infty}$ & free stream Mach No. \\
\hline $\mathrm{N}_{\mathrm{J} 1}$ & number of longitudinal joints \\
\hline $\mathrm{N}_{\mathrm{JP}}$ & number of peripheral joints \\
\hline $\mathrm{P}$ & panel lateral airload \\
\hline q & dynamic pressure \\
\hline$\dot{\mathrm{q}}$ & heating rate \\
\hline $\mathrm{R}$ & radius \\
\hline $\mathrm{T}$ & temperature \\
\hline $\mathrm{t}$ & thickness \\
\hline $\mathrm{t}_{\mathrm{J}}$ & joint gap width \\
\hline $\mathrm{x}_{\mathrm{P}}$ & periphery of vehicle section \\
\hline $\mathrm{X}_{1}$ & vehicle length \\
\hline$\alpha$ & coefficient of thermal expansion \\
\hline$\Delta \alpha$ & $\alpha$ (ablator) $-\alpha$ (substrate) \\
\hline
\end{tabular}




\section{NOMENCLATURE (continued)}

$\begin{array}{ll}\epsilon & \text { strain } \\ \mu & \text { Poisson's ratio } \\ \rho & \text { density } \\ \sigma & \text { stress }\end{array}$

Subscripts:

$\begin{array}{ll}\text { AB } & \text { ablator } \\ \text { cy } & \text { compression yield } \\ \mathrm{E} & \text { effective or equivalent } \\ \text { ins } & \text { insulation } \\ \mathrm{J} & \text { joint } \\ \mathrm{k} & \text { thermal conductivity } \\ \mathrm{L} & \text { local } \\ \text { min } & \text { minimum } \\ \text { max } & \text { maximum } \\ \mathrm{n} & \text { individual element } \\ \text { nom } & \text { nominal } \\ \mathrm{p} & \text { panel } \\ \mathrm{s} & \text { substrate } \\ \text { stag. } & \text { stagnation point } \\ \mathrm{t} & \text { thickness } \\ \text { ty } & \text { tensile yield } \\ \text { th } & \text { thermal } \\ \text { ult } & \text { ultimate } \\ \mathrm{y} & \text { yield }\end{array}$

PRELIMINARY SUBSTRATE CONCEPT AND MATERIAL SELECTION

Discussion of Candidate Concepts and Materials

This investigation was conducted in order to survey a broad spectrum of materials and structural concepts and, subsequently, to screen out the less desirable design combinations and arrive at a minimum number of thermostructural panel concepts for detailed optimization studies. 
In addition to a group of substrate panel structural concepts, the thermostructural panel was broken down into separate material components, and the most promising materials were selected from each group.

Substrate panel structural concepts. - The candidate substrate panel structural concepts are listed below and illustrated in Figure 2.

(1) Honeycomb panel

(2) Single and double skin corrugation panel

(3) Built-up stiffened skin panel

(4) Bead stiffened skin panel

(5) Machined waffle skin panel

A preliminary screening was conducted on the substrate concepts by comparing their relative merits with regard to reliability, structural efficiency, fabrication problems and reparability. A brief discussion of the concept comparisons in each of these areas follows:

Reliability: The panel concept(s) selected must be consistent with the requirement of high reliability. From a structural concept point of view (neglecting effects of materials) significant factors are: attachment techniques, degree of structural redundancy, and susceptibility to damage. Generally, all of the concepts appear competitive from a reliability point of view with the possible exception of the bead stiffened skin configuration. This concept offers a greater degree of damage susceptibility due to the possibility of a dent or crease in one of the thin skinned beads. Since the panel derives its rigidity from these beads and their spacing somewhat limits the degree of structural redundancy, its reliability rating should be somewhat less than that for the other concepts.

Structural efficiency: In considering the relative structural efficiencies, the type of loading the panel must carry was taken into consideration. The structural function of the substrate panel is to transmit local airloads to the basic vehicle structure, and therefore, the prime requirement is for flexural strength and rigidity as opposed to stability under an edge loading. Practical waffle and bead stiffened skin configurations can be shown efficient under edge loading conditions, but the need for a panel with moment carrying capability indicates the use of a double face panel (such as honeycomb) or a single face panel with deep or very closely spaced stiffeners. From a structural efficiency point of view, the waffle and bead stiffened configurations would appear inferior. 
Fabrication: A requirement of prime significance here is the need to fabricate the substrate panels to a compound contour. This requirement imposes some restrictions on the waffle, and skin-corrugation panels. Relatively speaking, the honeycomb panel, bead stiffened panel and built-up stiffened skin concepts offer fewer fabrication problems. A more detailed discussion of related fabrication problems is presented in the subsection on Manufacturing and Quality Control Review of Panel Concepts.

Reparability: The reparability of a panel is a function of the type of construction and extent of the damage. For a built-up stiffened skin panel, the damaged components may be readily removed and replaced, provided that the ablator has not yet been attached to the substrate. Similarly, in the absence of an ablative overlay, repair of the honeycomb, skin-corrugation, and bead stiffened panels would become increasingly difficult. Damage to a machined $\mathrm{rib}$ on the waffle configuration would pose a severe repair problem. The presence of ablator material on the substrate panels tends to equalize the relative reparability aspects of each of the panel concepts. Accordingly, for purposes of comparison, it was considered that no significant difference exists between the panels in terms of reparability.

In order to summarize the previous considerations in a convenient form, an evaluation chart has been generated which assigns a numerical rating to each concept in each category. Since not all of the factors have equal significance, a "significance factor" has been assigned to each one. The highest significance factor is 1.0 and is assigned to panel reliability. Significance factors have been selected for the other categories as follows:

Category

Reliability

Structural efficiency

Fabrication

Reparability
Significance factor

1.0

0.90

0.70

0.50

The basic numerical rating system is tabulated below:

$$
\begin{aligned}
& \text { Good - } 3 \\
& \text { Fair - } 2 \\
& \text { Poor - } 1
\end{aligned}
$$


The evaluation chart is shown in Table 1.

TABLE 1. PRELIMINARY RATING OF SUBSTRATE PANEL CONCEPTS

\begin{tabular}{|c|c|c|c|c|c|c|}
\hline Concept & Reliability & $\begin{array}{l}\text { Structural } \\
\text { efficiency }\end{array}$ & Fab. & Reparability & $\begin{array}{l}\text { Total } \\
\text { points }\end{array}$ & $\begin{array}{l}\text { \% of maximum } \\
\text { possible points }\end{array}$ \\
\hline Honeycomb & 3 & 2.7 & 2.1 & 1.0 & 8.8 & 94.5 \\
\hline $\begin{array}{l}\text { Skin- } \\
\text { corrugation }\end{array}$ & 3 & 2.7 & 1.4 & 1.0 & 8.1 & 87.0 \\
\hline $\begin{array}{l}\text { Built-up } \\
\text { stiffened } \\
\text { skin }\end{array}$ & 3 & 1.8 & 2.1 & 1.0 & 7.9 & 85.0 \\
\hline $\begin{array}{l}\text { Bead } \\
\text { stiffened } \\
\text { skin }\end{array}$ & 2 & 0.90 & 2.1 & 1.0 & 6.0 & 64.5 \\
\hline $\begin{array}{l}\text { Machined } \\
\text { waffle } \\
\text { skin }\end{array}$ & 3 & 1.8 & 1.4 & 1.0 & 7.2 & 77.5 \\
\hline
\end{tabular}

Based on the preliminary ratings established for each panel concept, the bead stiffened and waffle stiffened configurations were eliminated from further consideration. Preliminary consideration of built-up stiffened skin configurations shows that from a structural efficiency point of view this concept is best realized by a combination of small closely spaced stiffeners running in one direction and supported on widely spaced transverse stiffeners. This configuration in its ultimate form becomes analagous to a single skin corrugation panel supported on transverse stiffeners. The three most promising concepts then become:

(1) Honeycomb pane1

(2) Double skin corrugation panel

(3) Single skin corrugation panel

Prior to a detailed examination of these concepts it was necessary to select a group of promising materials for use in the thermostructural composite system.

Substrate panel materials. - The candidate substrate panel materials fall into two main categories: metals and plastic laminates. Specific materials investigated in each class are defined in Table 2. 
TABLE 2. CANDIDATE SUBSTRATE

PANEL MATERIALS

\begin{tabular}{|c|c|c|c|}
\hline \multirow[b]{2}{*}{ Metals } & \multicolumn{3}{|c|}{ Plastic laminates } \\
\hline & Resin designation & Reinforcement & Resin type \\
\hline $\begin{array}{l}17-7 \mathrm{PH} \mathrm{(TH} \\
1050) \text { stain- } \\
\text { less steel }\end{array}$ & CTL 91LD & $\begin{array}{l}181 \text { Weave } \mathrm{E} \\
\text { glass }\end{array}$ & Phenolic \\
\hline $\begin{array}{l}6 \mathrm{AL}-4 \mathrm{~V} \text { ti- } \\
\text { tanium }\end{array}$ & NARMCO 506 & $\begin{array}{l}181 \text { Weave } E \\
\text { glass }\end{array}$ & Phenolic \\
\hline $\begin{array}{l}\text { QMV cross } \\
\text { rolled beryl- } \\
\text { lium }\end{array}$ & CTL $37-9 x$ & $\begin{array}{l}181 \text { Weave } \mathrm{E} \\
\text { glass }\end{array}$ & $\begin{array}{l}\text { Phenyl- } \\
\text { silane }\end{array}$ \\
\hline $\begin{array}{l}2219-\mathrm{T} 87 \\
\text { aluminum }\end{array}$ & NARMCO 534 & $\begin{array}{l}181 \text { Weave } \mathrm{E} \\
\text { glass }\end{array}$ & $\begin{array}{l}\text { Phenyl- } \\
\text { silane }\end{array}$ \\
\hline \multirow[t]{3}{*}{ Lockalloy } & NARMCO 513 & $\begin{array}{l}181 \text { Weave } E \\
\text { glass }\end{array}$ & Silicone \\
\hline & DC 2106 & $\begin{array}{l}181 \text { Weave } E \\
\text { glass }\end{array}$ & Silicone \\
\hline & Imidite 1850 & $\begin{array}{l}1581 \text { Weave - } \\
994 \text { HTS glass }\end{array}$ & PBI \\
\hline
\end{tabular}

Comparative plots of $\mathrm{E} / \rho, \sigma_{\mathrm{ult}} / \rho$ and $\sigma_{\mathrm{y}} / \rho$ for the candidate metal substrate materials are shown in Figures 3 through 5 . Aluminum was eliminated from further consideration based on its inferior high-temperature performance. Lockalloy was also eliminated as a result of its inferior performance at elevated temperature, high cost (400-500 $\$ / 1 \mathrm{~b}, 882-1102 \$ / \mathrm{kg}$ ) and developmental status.

Of the reinforced plastic laminates (see figures 6 and 7 ), the silicone resin systems appear consistently inferior to the phenolic, phenyl-silane and PBI resin systems. From a stiffness standpoint, the Narmco 506 phenolic/glass, Narmco 534 and CTL 37-9x phenyl-silane/ glass and the Imidite 1850/glass systems each appear competitive across the expected operational temperature range. From the viewpoint of the strength properties of the laminates, the Imidite $1850 /$ glass laminate is superior to the other materials at all temperatures. Narmco $506 /$ glass displays good strength behavior up to $500^{\circ} \mathrm{F}\left(533^{\circ} \mathrm{K}\right)$ and then declines rapidly. The phenyl-silane/glass systems show excellent strength retention up to $1000^{\circ} \mathrm{F}\left(811^{\circ} \mathrm{K}\right)$, however, at room temperature 
their strength is only $60 \%$ of the Narmco 506 phenolic/glass system. Also included in Figure 7 are points defining strength properties of CTL-91LD resin and 181 weave "S" glass at room temperature and $500^{\circ} \mathrm{F}\left(533^{\circ} \mathrm{K}\right)$. An advantage is clearly indicated for " $\mathrm{S}$ " glass systems, and it is expected that their performance at higher temperatures will remain superior to the " $\mathrm{E}$ " glass reinforcements. The "S" glass is a relatively new development but is available on a production basis and is roughly equivalent to " $\mathrm{E}$ " glass on a cost basis. Sufficient material property data are not available to generate design allowable stress curves, and accordingly, for purposes of this study, "E" glass reinforcement has been considered.

Based on the above considerations, certain candidate materials were screened out of the study effort. A list of the remaining candidate substrate panel materials is contained in Table 3.

TABLE 3. CANDIDATE SUBSTRATE PANEL MATERIALS AFTER PRELIMINARY SCREENING

\begin{tabular}{|l||l|l|l|}
\hline \multicolumn{1}{|c||}{ Metals } & Resin designation & Reinforcement & Resin type \\
\hline $\begin{array}{l}17-7 \mathrm{PH} \text { (TH } \\
\text { 1050) stain- } \\
\text { less steel }\end{array}$ & NARMCO 506 & $\begin{array}{l}181 \text { Weave E } \\
\text { glass }\end{array}$ & Phenolic \\
$\begin{array}{l}\text { 6AL-4V ti- } \\
\text { tanium }\end{array}$ & CTL 37-9x & $\begin{array}{l}181 \text { Weave E } \\
\text { glass }\end{array}$ & $\begin{array}{l}\text { Phenyl- } \\
\text { silane }\end{array}$ \\
$\begin{array}{l}\text { QMV cross } \\
\text { rolled beryl- } \\
\text { lium }\end{array}$ & Imidite 1850 & $\begin{array}{l}1581 \text { Weave - } \\
994 \text { HTS glass }\end{array}$ & PBI \\
\hline
\end{tabular}

Adhesive bond materials. - Bonding materials may be used in the assembly of the substrate panels and for the attachment of the ablative material to the substrate. Selection of the bond material is critical since it may restrict the ablator back face temperature and can effect the thermal stresses generated in the composite panel. Since the permissible temperature at the ablator back face influences the heat shield weight, it is of prime importance to establish as high a temperature limit as the affected materials will permit. The candidate adhesive bond materials are listed below.

Adhesive bonds:

(1) $\mathrm{HT}-424$ 
(2) Narmco Imidite 850 (PBI)

(3) Epon 422

(4) Metlbond 302

(5) Metlbond 306

(6) Metlbond 329

(7) RTV silicon elastomers

Figure 8 shows the bond tensile shear strength versus temperature for the candidate adhesives. The Imidite 850 and HT-424 adhesives are clearly superior. Above $600^{\circ} \mathrm{F}\left(589^{\circ} \mathrm{K}\right)$ the Imidite 850 provides greater strength than the HT-424. Imidite 850 is a relatively new material, and, consequently, material performance data is not as widely confirmed as that for HT-424. Strengths for Imidite 850 quoted are based on a post cure cycle at $750^{\circ} \mathrm{F}\left(672^{\circ} \mathrm{K}\right)$ which is optional for stainless steel but must not be used for titanium. Lower post cure temperatures yield lower strength. Depending upon other materials used, the high cure temper atures could present other problems (degradation of plastic laminates, etc.). Based on the wealth of experience gained with the HT-424, it appears more favorable than the Imidite 850 from a reliability standpoint.

The ablator cold soak thermal stress problem can be partially relieved by incorporating a flexible bond between the ablator and substrate panel. RTV silicon rubber compounds are applicable here but are limited to $300^{\circ} \mathrm{F}\left(422^{\circ} \mathrm{K}\right)$ maximum bond temperatures, thereby imposing a severe weight penalty on the heat shield system.

The application of a mechanical attachment concept to the ablator/ substrate interface was briefly considered but discarded since such a scheme would require extensive development work which was beyond the scope of the program.

Insulation materials. - A group of promising insulation materials was surveyed; these materials are listed below:
(1) Microquartz
(2) Dynaquartz
(3) Q-felt
(4) Fiberfax Lo-Con
(5) Refrasil batt 

(6) Thermoflex
(7) Min-K 1301
(8) Santocel A
(9) Resistotherm

Figures 9 and 10 present data on comparative thermal diffusivity and $\rho \mathrm{k}$ values for the candidate materials. Multi-layer microquartz with aluminum foil radiation barriers was chosen as the insulation material. The basic material density is $6.21 \mathrm{lb} / \mathrm{ft}^{3}\left(99.48 \mathrm{~kg} / \mathrm{m}^{3}\right)$. The addition of five 0.0005 inch $(12.7 \mu \mathrm{m})$ aluminum foil layers per inch results in a composite density of $6.64 \mathrm{lb} / \mathrm{ft}^{3}\left(106.37 \mathrm{~kg} / \mathrm{m}^{3}\right)$. The upper temperature limit on the microquartz material for short exposure times is $3000^{\circ} \mathrm{F}\left(1922^{\circ} \mathrm{K}\right)$. The inclusion of the aluminum foil layers will reduce the maximum temperature to approximately $1000^{\circ} \mathrm{F}\left(811^{\circ} \mathrm{K}\right)$. Since the adhesive bond between the ablator and substrate and, in some cases, the substrate itself have lower temperature limits, this limiting insulation temperature imposes no constraints on the design. Thermal properties used for analysis of the microquartz insulation are presented in Figures 11 and 12 .

Fabrication problems associated with fitting this insulation material to complex shapes are minimal since the mat for $m$ of the insulation is very flexible.

Ablator materials. - Two candidate ablator materials were considered:

(1) NASA 602 elastomeric ablator

(2) Low density nylon phenolic (LDNP)

Property data of these materials are based on refs. 13 and 22 and are presented in Table 4 and Figures 13 through 21 . It should be noted here that both the thermochemical and mechanical properties of the se materials have been chosen as representative values. The elastomeric char density shown in Table 4 is associated with the inner portions of the char layer and does not account for char density buildup at the surface. Char density buildup at the surface is accounted for in the thermal analysis. 
TABLE 4. DENSITIES OF CANDIDATE ABLATORS

\begin{tabular}{|l|l|c|c|c|}
\hline \multirow{2}{*}{ Ablator } & \multicolumn{4}{|c|}{ Density } \\
\cline { 2 - 5 } & \multicolumn{3}{|c|}{ Virgin } & \multicolumn{2}{c|}{ Char } \\
\cline { 2 - 5 } & $1 \mathrm{~b} / \mathrm{ft}^{3}$ & $\mathrm{~kg} / \mathrm{m}^{3}$ & $1 \mathrm{~b} / \mathrm{ft}^{3}$ & $\mathrm{~kg} / \mathrm{m}^{3}$ \\
\hline LDNP & 36 & 576.7 & 15 & 240.3 \\
NASA 602 & 41.5 & 664.8 & 10 & 160.2 \\
\hline
\end{tabular}

From what is known of the silicone based elastomeric ablators, their applications should be restricted to heating rate ranges up to a maximum of 100 to $125 \mathrm{Btu} / \mathrm{ft}^{2}-\sec \left(1.135\right.$ to $\left.1.419 \mathrm{Mw} / \mathrm{m}^{2}\right)$ due to surface recession behavior. No data is available on the allowable heating rate ranges for LDNP, but test data indicates that in the heating rate range up to approximately $125 \mathrm{Btu} / \mathrm{ft}^{2}-\sec \left(1.419 \mathrm{Mw} / \mathrm{m}^{2}\right)$ the surface recession is somewhat higher than that for the elastomers. At higher heating rates, however, its behavior is superior to that of the elastomers. Insufficient data is available to develop a definition of the composite effects of enthalpy; pressure, shear, and heating rate on material performance which would make it possible to establish an oper ational envelope for the candidate ablator materials.

Significant design aspects related to the integration of each of the candidate ablators into a composite thermostructural panel system are discussed below.

Ablator material and process compatibility: The NASA 602 ablator can be applied to the substrate panel either with or without a honeycomb supporting matrix. Extensive experience has been acquired in injecting elastomeric ablators into honeycomb matrices which are bonded to substructures, and, though time consuming, no significant problems are associated with this technique. The application of an unsupported elastomeric ablator involves certain problems. It would first be necessary to premold the elastomeric ablator to the desired panel contours. Subsequently, attachment of the unsupported ablator to the substrate would require a compatible bond material. Adhe sive bond systems offering good strength retention at high temperatures, such as HT-424 and Imidite 850, provide poor bond strength with the silicone base elastomers and degrade the elastomer properties. An alternative consideration would be a silicone base adhesive such as RTV 560 elastomer, but practical experience with this material indicates a maximum bond temperature limit of $300^{\circ} \mathrm{F}\left(422^{\circ} \mathrm{K}\right)$. Furthermore, verification of the bond integrity would introduce quality control problems. An additional problem created by the omission of the honeycomb matrix is the resultant re- 
duction in char stability. It is possible that the addition of fillers such as quartz fibers could strengthen the char but the ablator attachment problem would still remain. Accordingly, the application of NASA 602 has been considered only with a honeycomb matrix. Flexible phenolic/ glass honeycomb matrices which are readily bonded to compound contoured surfaces have been developed. Open weave flexible matrices are currently being developed in order to provide a mechanical interlock between the filled ablator and matrix, reduce the matrix density and, in addition, reduce the thermal conductance of the ablator/matrix composite.

Because of the relatively brittle nature of LDNP, application of the LDNP ablator to the substrate panel with a flexible bond such as the RTV 560 silicone elastomer would appear to be advantageous. This would, in effect, structurally isolate the ablator from the substrate and, thereby, minimize the introduction of load induced stresses due to structural interaction and, in addition, tend to minimize the cold soak thermal stress problem. However, as mentioned previously, the low limiting temperature on the bond imposes a heat shield weight penalty. The remaining choice is a relatively rigid bond with a high temperature capability, such as HT-424. Limited experience in bonding LDNP with HT-424 resulted in severe shrinkage of the ablator at the required cure temperature $\left(350^{\circ} \mathrm{F}\right.$ or $450^{\circ} \mathrm{K}$ for $\left.1-1 / 2 \mathrm{hr}\right)$. It is apparent that extensive development work would be required in order to establish practical techniques for adhesive bonding LDNP to substrate panels.

Brief consideration was given to a mechanical attachment technique whereby a crushable honeycomb core material would be bonded to the substrate and, subsequently, the LDNP ablator would be molded integrally with the substrate panel, thereby resulting in a mechanical interlock. Prior development work has been conducted on this concept to a limited extent with phenolic/chopped glass as the ablator material. Further development work in this and related areas could evolve a practical ablator attachment scheme with an acceptable degree of reliability.

Geometry requirements: When the application to the HL-10 vehicle, or any of the current lifting body configurations is considered, the requirement for matching the ablator to a compound curved substrate panel must be satisfied. The flexible cores currently available are ideally suited to application in this area and much experience has been accumulated working with honeycomb supported elastomers on complex curved surfaces.

In the case of LDNP, it becomes obvious that, within the scope of current practical fabrication techniques, the ablator must be pre-molded to the desired contour and subsequently matched to the substrate panel 
in order to produce a reliable and homogeneous adhesive bond. It would be possible to use matching tools or, in some cases, to actually employ the substrate panel itself as a tool. However, the consideration of ablator shrinkage and differential thermal contraction during cure and the resultant panel warpage indicates potentially serious problems.

Reparability: The ablator panels will be susceptible to damage during handling and, therefore, a desirable feature for the ablative material would be its ease of repair.

The NASA 602 elastomeric ablator lends itself quite readily to on site repair. The damaged area can be cleaned out and new core and material added. Curing can be done at room temperature or by portable heat lamps.

The LDNP, being a pressure molding (100 - 200 psi or 689.5 $1379 \mathrm{kN} / \mathrm{m}^{2}$ ), is less amenable to field repair. Depending upon the extent of the damage, it might be possible to effect a repair by the use of plug type inserts or a castable version of the parent material. Some work has been done in the area of castable compounds of LDNP (ref.2); however, tests have shown the ablative performance of castable LDNP to be inferior to that of the pressure molded material.

\section{Analysis of Candidate Thermostructural Panel Concepts}

In the previous section, the more significant qualitative comparisons of the candidate concepts and materials have been defined. This section will discuss the results of the analysis which was conducted in order to establish some quantitative relationships among the various configurations that have passed the initial screening. For preliminary analytical studies involving the plastic laminate substrates, the phenolic/glass laminate thermal and mechanical properties were used. Thermal properties are shown in Figure 22 and Table 5. It was considered that this material was representative of the plastic laminate group.

TABLE 5. PHENOLIC/GLASS LAMINATE THERMAL PROPERTIES

\begin{tabular}{|c|c|c|c|c|}
\hline \multicolumn{2}{|c|}{ Density } & \multicolumn{2}{c|}{ Specific heat } & \multirow{2}{*}{ Emissivity } \\
\cline { 1 - 3 } $\mathrm{1b} / \mathrm{in}^{3}$ & $\mathrm{gm} / \mathrm{cm}^{3}$ & $\mathrm{Btu} / \mathrm{lb}^{-} \mathrm{K}$ & $\mathrm{J} / \mathrm{kg}^{-} \mathrm{K}$ & \\
\hline 0.06 & 1.661 & 0.28 & 1171.5 & 0.80 \\
\hline
\end{tabular}


Comparison of heat shield weights. - In order to evaluate the relative efficiencies of the NASA 602 elastomeric ablator and the LDNP ablator, an idealized re-entry heating pulse for a typical entry vehicle was established as a basis for analysis; it is defined in Figure 23. Total heat shield weights were calculated for a vehicle with an assumed reference length of $25 \mathrm{ft}(7.62 \mathrm{~m})$. The total wetted area of the vehicle was taken to be $727 \mathrm{ft}^{2}\left(67.54 \mathrm{~m}^{2}\right)$. In addition, an estimated average heating rate over the entire vehicle was established as $0.0875 \dot{\mathrm{q}}_{\mathrm{stag}}$ : The analytical model is defined below:

Ablator:

Substrate panel:

Insulation:

Structural backface:

Temperature constraints:
NASA 602 or LDNP

Phenolic/glass honeycomb face $\mathrm{t}=0.02$ in. $(0.508 \mathrm{~mm}) 5.5$ $\mathrm{lb} / \mathrm{ft}^{3}\left(88.1 \mathrm{~kg} / \mathrm{m}^{3}\right)$ Hexcel HRP core Total depth - $0.25 \mathrm{in.}$ (6. $35 \mathrm{~mm}$ )

$6.64 \mathrm{lb} / \mathrm{ft}^{3}\left(106.37 \mathrm{~kg} / \mathrm{m}^{3}\right)$ microquartz/aluminum foil

Aluminum 0.05 in. $(1.27 \mathrm{~mm})$ thickness

Maximum bond temperature $800^{\circ} \mathrm{F}\left(700^{\circ} \mathrm{K}\right)$ Maximum structural backface temperature $150^{\circ} \mathrm{F}\left(339^{\circ} \mathrm{K}\right)$

The analysis was performed on the Martin T-CAP III digital program, which is described in Appendix A. The resultant heat shield weights (ablator + insulation + substrate) are tabulated in Table 6.

TABLE 6. COMPARATIVE HEAT SHIELD WEIGHTS AND THICKNESSES

\begin{tabular}{|l|c|c|c|c|}
\hline \multirow{2}{*}{ Ablator } & \multicolumn{2}{|c|}{ Total heat shield weight } & \multicolumn{2}{c|}{ Composite thickness } \\
\cline { 2 - 5 } & $\mathrm{lb}$ & $\mathrm{kg}$ & in. & $\mathrm{mm}$ \\
\hline NASA 602 & 2465 & 1118 & 2.16 & 54.9 \\
LDNP & 2208 & 1002 & 2.15 & 54.6 \\
\hline
\end{tabular}


It can be seen that the LDNP heat shield system weight is $257 \mathrm{lb}$ $(116 \mathrm{~kg})$ lighter than the NASA 602 system. This amounts to a weight saving of approximately $10 \%$, which is significant.

Data generated in the heat shield weight comparisons were also used to develop a curve which defines the effect of bond temperature constraint on the required weights for both of the candidate ablators. Curves defining this relationship are presented in Figure 24. As the limiting bond temperature decreases, the increased ablator weight would be partially off set by a decrease in the required insulation weight.

Thermal stress considerations. - An analytical model was established as a basis for comparison of the relative performance of the various combinations of ablator and substrate materials in a typical orbital cold soak environment. The model is defined as follows:

(1) 1.25 in. ( $31.8 \mathrm{~mm}$ ) ablator material is bonded to a homogeneous substrate plate of thickness $\left(t_{s}\right)$

(2) Adhesive bond is rigid

(3) All materials are isotropic

(4) Composite panel is soaked at a uniform temperature of $-110^{\circ} \mathrm{F}\left(194^{\circ} \mathrm{K}\right)$

(5) Composite panel is free to expand but restrained against curvature

(6) Thermoelastic behavior prevails

(7) No thermal buckling occurs

The governing equation for biaxial thermal strain in the ablator becomes:

$$
\epsilon_{A B}=\frac{\Delta \alpha \Delta T}{1-\mu_{A B}}\left[\frac{E_{s} t_{s}}{E_{s} t_{s}+E_{A B} t_{A B}\left(\frac{1-\mu_{s}}{1-\mu_{A B}}\right)}\right]
$$

For the composite ablator panels under consideration, the evaluation of thermal stresses requires a consideration of fabrication processes unique to each of the configurations. The LDNP ablator is bonded and cured to the substrate panel at a temperature of $350^{\circ} \mathrm{F}\left(450^{\circ} \mathrm{K}\right)$ with H T-424 adhesive. Accordingly, the base temperature for thermal stress analysis is $350^{\circ} \mathrm{F}\left(450^{\circ} \mathrm{K}\right)$. In the case of the NASA 602 ablator, the honeycomb matrix is first bonded to the substrate and subsequently filled with the ablator material. The entire panel is then cured at $230^{\circ} \mathrm{F}\left(383^{\circ} \mathrm{K}\right)$ which becomes the base temperature for thermal stress. 
Table 7 summarizes the pertinent material properties used in the analysis. The thermal expansion coefficients for the ablator materials are averaged over the applicable temperature ranges.

\section{TABLE 7. MATERIAL PROPERTIES FOR COLD SOAK THERMAL STRESS COMPARISON}

\begin{tabular}{|c|c|c|c|c|c|c|c|c|}
\hline \multirow[b]{2}{*}{ Material } & \multicolumn{2}{|c|}{$\alpha \times 10^{6}$} & \multicolumn{2}{|c|}{$E \times 10^{-6}$} & \multirow[b]{2}{*}{$\mu$} & \multirow[b]{2}{*}{$\epsilon_{\text {ult }}$} & \multicolumn{2}{|c|}{$\Delta \mathbf{T}$} \\
\hline & in. $/$ in. ${ }^{\circ} \mathrm{F}$ & $\mathrm{cm} / \mathrm{cm}^{\circ} \mathrm{K}$ & psi & $\mathrm{kN} / \mathrm{m}^{2}$ & & & ${ }^{\circ} \mathrm{F}$ & ${ }^{\circ} \mathrm{K}$ \\
\hline LDNP & 25.6 & 46.1 & 0.145 & 1.0 & 0.25 & 0.0082 & -460 & -256 \\
\hline NASA 602 & 56.3 & 101.3 & 0.084 & 0.579 & 0.30 & 0.021 & -340 & -189 \\
\hline $\begin{array}{l}\text { Stainless } \\
\text { steel }\end{array}$ & 6.1 & 11.0 & 28.5 & 196.5 & 0.28 & & & \\
\hline Titanium & 4.8 & 8.6 & 16 & 110.3 & 0.33 & & & \\
\hline Beryllium & 6.0 & 10.8 & 43.5 & 299.9 & 0.03 & & & \\
\hline $\begin{array}{l}\text { Phenolic/ } \\
\text { glass }\end{array}$ & 4.9 & 8.8 & 4. 3 & 29.6 & 0.16 & & & \\
\hline
\end{tabular}

Results of the analysis are plotted in Figure 25. The curves show the thermal stresses in the ablator and substrate panels as a function of the substrate panel thickness. It can be seen that for a given substrate thickness the higher stresses occur in the NASA 602 ablator materials and substrate panels. It should also be noted that the allowable stress of the NASA 602 is higher than that for the LDNP. It will be recalled that the analytical model used here is based on elastic analysis. An inelastic thermal stress analysis conducted on both $a b-$ lators would yield results still more favorable to the NASA 602 elastomeric ablator due to its greater flexibility at low temperatures.

Based on the analysis, a set of substrate panel thickness constraints can be determined on the basis of material allowable stresses; they are presented in Table 8. 
TABLE 8. SUBSTRATE PANEL THICKNESS CONSTRAINTS FOR THERMAL STRESS COMPATIBILITY

\begin{tabular}{|c|c|c|c|c|c|c|c|c|}
\hline \multirow{3}{*}{$\begin{array}{l}\text { Substrate } \\
\text { material }\end{array}$} & \multicolumn{4}{|c|}{$\begin{array}{l}\text { Low density } \\
\text { nylon phenolic }\end{array}$} & \multicolumn{4}{|c|}{ NASA 602} \\
\hline & \multicolumn{2}{|c|}{$\operatorname{Max} t_{s}$} & \multicolumn{2}{|c|}{$\operatorname{Min} t_{s}$} & \multicolumn{2}{|c|}{$\operatorname{Max} t_{s}$} & \multicolumn{2}{|c|}{ Min $t_{s}$} \\
\hline & in. & $\mathrm{mm}$ & in. & $\mathrm{mm}_{1}$ & in. & $\mathrm{mm}$ & in. & $\mathrm{mm}$ \\
\hline $\begin{array}{l}\text { Stainless } \\
\text { steel }\end{array}$ & 0.013 & 0.330 & 0.004 & 0.102 & 0.024 & 0.610 & 0.009 & 0.229 \\
\hline Titanium & 0.017 & 0.432 & 0.004 & 0.102 & 0.035 & 0.889 & 0.009 & 0.229 \\
\hline Beryllium & $0.011 *$ & 0.279 & $0.023 *$ & 0.584 & $0.020 *$ & 0.508 & $0.030 *$ & 0.762 \\
\hline $\begin{array}{l}\text { Phenolic/ } \\
\text { glass }\end{array}$ & 0.084 & 0.213 & 0.001 & 0.025 & 0.154 & 3. 912 & 0.018 & 0.457 \\
\hline
\end{tabular}

*For $t_{s} \leqq 0.023$ in. $(0.584 \mathrm{~mm})$ beryllium fails, for $t_{s} \geq 0.011$ in. $(0.279 \mathrm{~mm})$ LDNP fails. For $t_{s} \leqq 0.030$ in. $(0.762 \mathrm{~mm})$ beryllium fails, for $t_{s} \geqq 0.020$ in. $(0.508 \mathrm{~mm}$ ) NASA 602 fails.

If the substrate thickness exceeds max $t_{s}$, a failure is induced in the ablator material. Conversely, a failure occurs in the substrate panel $\left(\sigma_{\text {cy }}=\sigma_{\text {th }}\right)$ if substrate thicknesses less than min $\mathrm{t}_{\mathrm{s}}$ are used. It is assumed that the bond strength is adequate. The use of a flexible bond material will increase the substrate thickness range.

Of the ablative materials considered, NASA 602 is less critical from a thermal stress standpoint than LDNP. The substrate materials may be listed in the order of preference regarding their thermal stress characteristics as follows:

(1) Phenolic/glass laminate

(2) Titanium

(3) Stainless steel

(4) Beryllium

The low stiffness of the plastic laminate substrate minimizes thermal stresses in the composite panel. Titanium and stainless steel are in the same class from a thermal stress point of view with titanium having a slight advantage. The beryllium substrate material is incompatible with either of the ablator materials for the case considered unless a flexible bond is employed. 
Comparison of substrate panel structural efficiencies. - The relative structural efficiencies of the honeycomb and single and double skin corrugation concepts in combination with the more promising substrate materials were determined for a specific analytical model as defined below:

(1) $24 \times 24$ in. $(0.61 \times 0.61 \mathrm{~m})$ flat panel

(2) Four post supports are symmetrically located, free to rotate, capable of load transfer normal and parallel to the panel surfaces. Supports are located at panel locations so as to provide minimal deflections and moments for each concept considered

(3) All edges are free

(4) Ablator is nonstructural

For all honeycomb configurations, a $5.5 \mathrm{lb} / \mathrm{ft}^{3}\left(88.1 \mathrm{~kg} / \mathrm{m}^{3}\right)$ phenolic/ glass core is assumed. The weight of the HT-424 adhesive bond is $0.14 \mathrm{lb} / \mathrm{ft}^{2}\left(0.683 \mathrm{~kg} / \mathrm{m}^{2}\right)$ per layer. Two bond layers are used in the honeycomb and double skin corrugation panel and one layer is used in the single skin corrugation panel.

Both room and elevated temperature conditions were considered. The panel loadings are defined for both cases as:

Case

Room temperature

Elevated temperature $\underline{\text { Panel loading }}$

4 psig $\left(27.56 \mathrm{kN} / \mathrm{m}^{2}\right)$

$2 \operatorname{psig}\left(13.78 \mathrm{kN} / \mathrm{m}^{2}\right)$

The optimum panel weight for each concept and material combination was determined within the following constraints:

(1) Maximum permissible panel deflection $=0.125 \mathrm{in}$. $(3.175 \mathrm{~mm}$ )

(2) Maximum permissible ablator strain for a 1.25 in. $(31.75 \mathrm{~mm})$ thick ablator $=0.7 \%$

(This limit was applied only to the room temperature case. For elevated temperatures, no ablator strain constraint was imposed. ) 
(3) The substrate stresses shall not exceed ultimate allowables for the substrate materials, and panel element stability shall be maintained.

(4) Optimum panel weights must be based on realistic material gages both from a fabrication and material availability standpoint. Minimum gages are listed in Table 9.

TABLE 9. MINIMUM GAGES FOR CANDIDATE SUBSTRATE PANEL MATERIALS

\begin{tabular}{|l|l|c|}
\hline \multirow{2}{*}{\multicolumn{1}{|c|}{ Material }} & \multicolumn{2}{c|}{ Minimum gage } \\
\cline { 2 - 3 } Stainless steel & 0.0015 & $\mathrm{~mm}$ \\
Titanium & 0.015 & 0.0381 \\
Beryllium & 0.01 & 0.381 \\
Phenolic/glass laminate & 0.02 & 0.254 \\
\end{tabular}

A 1620 computer program was developed in order to determine the optimum structural configurations for the honeycomb and skin-corrugation configurations. Logic diagrams for the honeycomb and single skin corrugation programs are shown in Figures 26 and 27. For the single skin corrugation, a transverse supporting beam was optimized at a maximum permissible depth of 0.75 in. $(19.0 \mathrm{~mm})$ in order to remain compatible with the expected insulation thicknesses. The double skin corrugation program was similar to that for the single skin version with the exceptions that no transverse supporting beams were considered, and the panel was assumed to have isotropic properties.

Figures 28 through 39 present the results of the optimization study for each concept and material combination. It is seen that, within the bounds imposed by the minimum gage constraint, the room temperature design case dictates the optimum panel weight. The stainless steel substrates are the only group whose optimum weight is not influenced by the minimum gage constraint. The titanium panel optimum weights are severely penalized by the minimum gage constraint, the penalty becoming greater respectively with the honeycomb, single skin corrugation, and double skin corrugation panels. In the case of the beryllium substrates, the honeycomb panel optimum weight is increased only slightly, but the excellent weight potential of this material is more noticeably affected in the single and double skin corrugation concepts by the minimum gage constraint. Similarly, the plastic laminate 
group, as represented by Narmco 506 phenolic/glass reinforcement is relatively insensitive to the minimum thickness constraint in the honeycomb panel configuration but suffers an increasing penalty as the concept is changed to single and then double skin corrugation.

For convenience of comparing the effect of substrate material on a given panel structural concept, Figures 40 through 42 are compilations of the optimum weight curves for the room temperature design case. Table 10 compares the optimum weights of each configuration and defines the applicable constraint for the room temperature case.

TABLE 10. SUBSTRATE PANEL OPTIMUM WEIGHT COMPARISONS AT ROOM TEMPERATURE

\begin{tabular}{|c|c|c|c|c|}
\hline \multirow[t]{2}{*}{ Concept } & \multirow[t]{2}{*}{ Material } & \multicolumn{2}{|c|}{ Optimum weight } & \multirow[t]{2}{*}{ Constraint } \\
\hline & & $\mathrm{lb} / \mathrm{ft}^{2}$ & $\mathrm{~kg} / \mathrm{m}^{2}$ & \\
\hline \multirow[t]{4}{*}{ Honeycomb } & Steel & 0.83 & 4.05 & Ablator strain \\
\hline & Titanium & 1.18 & 5.76 & Minimum gage \\
\hline & Beryllium & 0.63 & 3.07 & Minimum gage \\
\hline & $\begin{array}{l}\text { Plastic } \\
\text { laminate }\end{array}$ & 0.94 & 4.59 & Minimum gage \\
\hline \multirow[t]{4}{*}{$\begin{array}{l}\text { Single skin } \\
\text { corrugation }\end{array}$} & Steel & 0.88 & 4. 29 & $\begin{array}{l}\text { Ablator strain/ } \\
\text { skin stability }\end{array}$ \\
\hline & Titanium & 1.53 & 7.47 & Minimum gage \\
\hline & Beryllium & 0.58 & 2.83 & Minimum gage \\
\hline & $\begin{array}{l}\text { Plastic } \\
\text { laminate }\end{array}$ & 0.86 & 4. 20 & Minimum gage \\
\hline \multirow[t]{4}{*}{$\begin{array}{l}\text { Double skin } \\
\text { corrugation }\end{array}$} & Steel & 0.91 & 4. 44 & $\begin{array}{l}\text { Ablator strain } \\
\text { skin stability }\end{array}$ \\
\hline & Titanium & 2.06 & 10.05 & Minimum gage \\
\hline & Beryllium & 0.82 & 4. 00 & Minimum gage \\
\hline & $\begin{array}{l}\text { Plastic } \\
\text { laminate }\end{array}$ & 1.17 & 5.71 & Minimum gage \\
\hline
\end{tabular}


It can be seen that for steel and titanium substrate materials the lightest panel weight occurs in the honeycomb configuration. However, for beryllium and plastic laminates, the single skin corrugation panel offers a slight weight advantage. This crossover is the result of including the adhesive bond weights for each concept. When panel weights exclusive of bond layer weights are considered, the honeycomb configuration is superior for all materials. Adhesive bond weights add $0.28 \mathrm{lb} / \mathrm{ft}^{2}\left(1.37 \mathrm{~kg} / \mathrm{m}^{2}\right)$ to the honeycomb panel and $0.14 \mathrm{lb} / \mathrm{ft}^{2}$ $\left(0.68 \mathrm{~kg} / \mathrm{m}^{2}\right)$ to the single skin corrugation concept. Consideration of the panel geometry required for the plastic laminate single skin corrugation reveals the skin and corrugations to be 0.02 in. $(0.508 \mathrm{~mm})$ thick and the corrugation depth and pitch to be 0.625 in. $(15.9 \mathrm{~mm})$. As indicated in the following subsection, fabrication of this configuration is impractical. An additional structural consideration pertinent to the single skin corrugation panels is that for the optimum panel weight and material, the skin corrugation concept has 50\% greater axial stiffness than does the honeycomb panel. This results in an increased pickup of structural loads from the vehicle primary structure in the case of rigid panel supports and, therefore, increased problems with the strain sensitive ablators. For the same reason, ablator thermal stresses will be greater with skin corrugation panels at the location of supporting transverse beams. The use of single skin corrugation panels could impose more stringent requirements on local panel strength at point support locations relative to honeycomb panels.

\section{Manufacturing and Quality Control Review of Panel Concepts}

A review of the fabrication aspects of the thermostructural panel concepts indicates the best configuration to be the NASA 602 elastomeric ablator bonded to a plastic laminate honeycomb substrate panel.

Relative to the two ablator materials, consideration of the ablator to substrate interface problem and tooling costs indicates a clear advantage for the NASA 602 elastomeric ablator.

The honeycomb substrate selection was based on consideration of fabrication costs, degree of repeatability, tolerances, and general ease of fabrication.

Tables 11a through $11 \mathrm{e}$ summarize pertinent fabrication factors for the various substrate panel configurations considered. Limitations are based on the premise that the panels must conform to a compound curvature. Beryllium was not included in these comparisons because of its inherent difficulties relative to fabrication (forming, machining and handling). Practical applications of beryllium would be limited to flat or single degree of curvature honeycomb panels. 
It is seen that the use of titanium in any structural configuration entails forming problems (heated tools) and a more severe restriction on curvature than that which is associated with steel. The advantages of plastic laminates over the metallic substrate materials are clearly evident when considering mold line tolerances and curvature limitations.

The results of the structural optimization analysis indicate that the single skin corrugation and honeycomb substrate panels are competitive on a structural efficiency basis and that for the plastic laminate configurations, the single skin corrugation has a slight weight advantage. However, consideration of the plastic laminate corrugation fabrication problem indicates that, for the material thicknesses and geometry associated with the optimum panel weight, it is impractical to manufacture, (see Table 11b). An additional fabrication problem is related to the skin-corrugation configurations in that when bonding ablators or flex-core matrices to the substrate surface, bonding pressures will vary between the corrugation cresis and the unsupported face sheet areas between corrugation crests. This problem also applies in varying degrees to all the concepts except honeycomb. Development work would be required in this area to define the magnitude of the problem and generate a practical solution, such as temporary fillers for panel skin support.

On the basis of the considerations outlined in Tables $11 \mathrm{a}$ thru $11 \mathrm{e}$, the substrate configurations have been rated in order of preference from a fabrication point of view and are listed below in that order:

(1) Plastic laminate honeycomb

(2) Stainless steel honeycomb

(3) Plastic laminate bead stiffened skin

(4) Stainless steel bead stiffened skin

(5) Titanium honeycomb

(6) Titanium bead stiffened skin

(7) Stainless steel built-up stiffened skin

(8) Stainless steel skin-corrugation

(9) Plastic laminate built-up stiffened skin

(10) Titanium built-up stiffened skin

(11) Plastic laminate corrugation (in practical sizes)

(12) Titanium corrugation

(13) Stainless steel machined waffle skin

(14) Titanium machined waffle skin 
TABLE 11a. SUMMARY OF FABRICATION FACTORS - HONEYCOMB SUBSTRATE PANEL

\begin{tabular}{|c|c|c|c|c|c|c|c|c|}
\hline \multirow[t]{2}{*}{ Item } & \multicolumn{2}{|c|}{$\begin{array}{l}\text { Minimum } \\
\text { gage }\end{array}$} & \multicolumn{2}{|c|}{$\begin{array}{l}\text { *Curvature } \\
\text { restrictions }\end{array}$} & \multirow[t]{2}{*}{ Tooling } & \multirow[t]{2}{*}{$\begin{array}{l}\text { Tool heat- } \\
\text { ing method }\end{array}$} & \multirow[t]{2}{*}{ Tolerance } & \multirow[t]{2}{*}{ Remarks } \\
\hline & in. & $\mathrm{mm}$ & in. /in. & $\mathrm{m} / \mathrm{m}$ & & & & \\
\hline $\begin{array}{l}\text { Titanium } \\
\text { facings }\end{array}$ & $\begin{array}{l}0.020 \\
\text { Limited } \\
\text { forming }\end{array}$ & $\begin{array}{l}0.508 \\
y \text { stretch }\end{array}$ & $36 / 3$ & $0.91 / 0.076$ & $\begin{array}{l}\text { Steel } \\
\text { stretch } \\
\text { block }\end{array}$ & $\begin{array}{l}\text { Radiant } \\
\text { heat lamp }\end{array}$ & $\begin{array}{l}0.062-\text { in. }(1.57 \mathrm{~mm}) \\
\text { on mold line }\end{array}$ & $\begin{array}{l}\text { Development of spring } \\
\text { back could reduce } \\
\text { tolerance to } 0.02 \text { in. } \\
(0.508 \mathrm{~mm}) \text { (costly) }\end{array}$ \\
\hline $\begin{array}{l}\text { Steel } \\
\text { facings }\end{array}$ & $\begin{array}{l}0.020 \\
\text { Limited } \\
\text { forming }\end{array}$ & $\begin{array}{l}0.508 \\
\text { y stretch }\end{array}$ & $36 / 6$ & $0.91 / 0.152$ & $\begin{array}{l}\text { Plastic } \\
\text { stretch } \\
\text { block }\end{array}$ & $\begin{array}{l}\text { None } \\
\text { required }\end{array}$ & Same as above & Same as above \\
\hline $\begin{array}{l}\text { Plastic } \\
\text { laminate } \\
\text { facings }\end{array}$ & $\begin{array}{l}0.020 \\
\text { Limited } \\
\text { availabil } \\
\text { structurs }\end{array}$ & $\begin{array}{l}0.508 \\
\text { y material } \\
\text { ty and } \\
1 \text { design }\end{array}$ & No limit & & $\begin{array}{l}\text { Male } \\
\text { metal } \\
\text { block- } \\
\text { vacuum } \\
\text { bag }\end{array}$ & Autoclave & $\begin{array}{l}\text { Mold line } \\
\text { as good } \\
\text { as tool }\end{array}$ & $\begin{array}{l}\text { Accurate mold line } \\
\text { tolerance. Excellent } \\
\text { adaptability to com- } \\
\text { pound contours }\end{array}$ \\
\hline $\begin{array}{l}\text { Phenolic/ } \\
\text { glass } \\
\text { honeycomb } \\
\text { core }\end{array}$ & $\begin{array}{l}3 / 16-\mathrm{in} . \\
\text { cell } \\
5.5 \\
\mathrm{lb} / \mathrm{ft}^{3}\end{array}$ & $\begin{array}{l}4.76 \mathrm{~mm} \\
\text { cell } \\
88.11 \\
\mathrm{~kg} / \mathrm{m}^{3}\end{array}$ & $\begin{array}{l}18 \text {-in. } R \\
\text { across } \\
\text { ribbon } \\
36 \text {-in. } R \\
\text { along } \\
\text { ribbon }\end{array}$ & $\begin{array}{l}0.457-\mathrm{m} \\
\mathrm{R} \text { across } \\
\text { ribbon } \\
0.91-\mathrm{m} \\
\mathrm{R} \text { along } \\
\text { ribbon }\end{array}$ & $\begin{array}{l}\text { Metal } \\
\text { bond fix- } \\
\text { ture vac- } \\
\text { uum bag }\end{array}$ & Autoclave & $\begin{array}{l} \pm 0.015 \mathrm{in} .( \pm \\
0.38 \mathrm{~mm}) \text { on } \\
\text { thickness }\end{array}$ & \\
\hline
\end{tabular}

*Note: Curvature restrictions in Table 11 are shown as a ratio of the side dimension of a square panel to the maximum rise. 
TABLE 11b. SUMMARY OF FABRICATION FACTORS-SKIN CORRUGATION SUBSTRATE PANELS

\begin{tabular}{|c|c|c|c|c|c|c|c|c|}
\hline \multirow[t]{2}{*}{ Item } & \multicolumn{2}{|c|}{$\begin{array}{l}\text { Minimum } \\
\text { gage }\end{array}$} & \multicolumn{2}{|c|}{$\begin{array}{c}\text { Curvature } \\
\text { restrictions }\end{array}$} & \multirow[t]{2}{*}{ Tooling } & \multirow[t]{2}{*}{$\begin{array}{l}\text { Tool heating } \\
\text { method }\end{array}$} & \multirow[t]{2}{*}{ Tolerance } & \multirow[t]{2}{*}{ Remarks } \\
\hline & in. & $\mathrm{mm}$ & in. /in. & $\mathrm{m} / \mathrm{m}$ & & & & \\
\hline $\begin{array}{l}\text { Skin } \\
\text { sheets }\end{array}$ & -- & -- & -- & -- & - & - & -- & $\begin{array}{l}\text { See Table 11a--Honey- } \\
\text { comb panel facing notes } \\
\text { apply here }\end{array}$ \\
\hline $\begin{array}{l}\text { Titanium } \\
\text { corruga- } \\
\text { tion }\end{array}$ & $\begin{array}{l}0.02 \\
\text { Limited } \\
\text { forming }\end{array}$ & $\begin{array}{c}0.508 \\
\text { by stretch }\end{array}$ & $24 / 2$ & $\begin{array}{l}0.609 / \\
0.0508\end{array}$ & $\begin{array}{l}\text { Matched } \\
\text { metal } \\
\text { dies - } \\
\text { stress } \\
\text { relief } \\
\text { fixture }\end{array}$ & $\begin{array}{l}\text { Electrically } \\
\text { heated } 600^{\circ} \mathrm{F} \\
\left(589^{\circ} \mathrm{K}\right)\end{array}$ & $\begin{array}{l}0.032 \mathrm{in.} \\
(0.813 \mathrm{~mm}) \\
\text { on mold line }\end{array}$ & Very costly operation \\
\hline $\begin{array}{l}\text { Steel } \\
\text { corruga - } \\
\text { tion }\end{array}$ & $\begin{array}{l}0.02 \\
\text { Limited } \\
\text { forming }\end{array}$ & $\begin{array}{l}0.508 \\
\text { by stretch }\end{array}$ & $36 / 3$ & $\begin{array}{l}0.91 / \\
0.076\end{array}$ & $\begin{array}{l}\text { Matched } \\
\text { metal } \\
\text { dies }\end{array}$ & $\begin{array}{l}\text { None re- } \\
\text { quired }\end{array}$ & $\begin{array}{l}\text { Same as } \\
\text { above }\end{array}$ & \\
\hline $\begin{array}{l}\text { Plastic } \\
\text { laminate } \\
\text { corruga- } \\
\text { tion }\end{array}$ & $\begin{array}{l}0.02 \\
\text { Limited } \\
\text { availabl } \\
\text { structur }\end{array}$ & $\begin{array}{l}\quad 0.508 \\
\text { by material } \\
\text { e and } \\
\text { al design }\end{array}$ & No limit & & - & -- & -- & $\begin{array}{l}\text { Not practical in di- } \\
\text { mensional and geometric } \\
\text { range considered. Ma- } \\
\text { terial will tear at radii }\end{array}$ \\
\hline
\end{tabular}


TABLE 11c. SUMMARY OF FABRICATION FACTORS -BUILT - UP STIFFENED SKIN SUBSTRATE PANEL

\begin{tabular}{|c|c|c|c|c|c|c|c|c|}
\hline \multirow[b]{2}{*}{ Item } & \multicolumn{2}{|c|}{$\begin{array}{l}\text { Minimum } \\
\text { gage }\end{array}$} & \multicolumn{2}{|c|}{$\begin{array}{c}\text { Curvature } \\
\text { restrictions }\end{array}$} & \multirow[b]{2}{*}{ Tooling } & \multirow{2}{*}{$\begin{array}{l}\text { Tool heating } \\
\text { method }\end{array}$} & \multirow[b]{2}{*}{ Tolerance } & \multirow[b]{2}{*}{ Remarks } \\
\hline & in. & $\mathrm{mm}$ & in. / in. & $\mathrm{m} / \mathrm{m}$ & & & & \\
\hline $\begin{array}{l}\text { Titanium } \\
\text { skin }\end{array}$ & $\begin{array}{l}0.020 \\
\text { Limited }\end{array}$ & $\begin{array}{c}0.508 \\
\text { oy forming }\end{array}$ & $36 / 3$ & $0.91 / 0.076$ & $\begin{array}{l}\text { Steel } \\
\text { marform } \\
\text { die - } \\
\text { stress } \\
\text { relief } \\
\text { fixture }\end{array}$ & $\begin{array}{l}\text { Radiant } \\
\text { heat lamp }\end{array}$ & $\begin{array}{l}0.032 \text { in. }(0.813 \\
\text { mm) on mold } \\
\text { line }\end{array}$ & Minimum bend radii of $2 \mathrm{t}$ \\
\hline $\begin{array}{l}\text { Titanium } \\
\text { hat } \\
\text { sections }\end{array}$ & $\begin{array}{l}0.020 \\
\text { Limited }\end{array}$ & $\begin{array}{c}0.508 \\
\text { oy forming }\end{array}$ & -- & -- & $\begin{array}{l}\text { Standard } \\
\text { tools - } \\
\text { steel } \\
\text { stretch } \\
\text { blocks }\end{array}$ & $\begin{array}{l}\text { Resistance } \\
\text { heat }\end{array}$ & Same as above & -- \\
\hline $\begin{array}{l}\text { Steel } \\
\text { skin }\end{array}$ & $\begin{array}{l}0.020 \\
\text { Limited }\end{array}$ & $\begin{array}{l}0.508 \\
\text { by forming }\end{array}$ & $36 / 4$ & $0.91 / 0.102$ & $\begin{array}{l}\text { Steel } \\
\text { marform } \\
\text { die }\end{array}$ & $\begin{array}{l}\text { None re- } \\
\text { quired }\end{array}$ & Same as above & $\begin{array}{l}\text { Heat treat in retort or } \\
\text { hydrogen bell furnace }\end{array}$ \\
\hline $\begin{array}{l}\text { Steel } \\
\text { hat } \\
\text { sections }\end{array}$ & $\begin{array}{l}0.02 \\
\text { Limited }\end{array}$ & $\begin{array}{l}0.508 \\
\text { by forming }\end{array}$ & - & -- & $\begin{array}{l}\text { Standard } \\
\text { tools - } \\
\text { stretch } \\
\text { blocks }\end{array}$ & $\begin{array}{l}\text { None re- } \\
\text { quired }\end{array}$ & Same as above & Same as above \\
\hline $\begin{array}{l}\text { Plastic } \\
\text { laminate } \\
\text { skin }\end{array}$ & $\begin{array}{l}0.02 \\
\text { Limited } \\
\text { available } \\
\text { structur }\end{array}$ & $\begin{array}{l}0.508 \\
\text { oy material } \\
\text { and } \\
\text { in design }\end{array}$ & No limi & & $\begin{array}{l}\text { Female } \\
\text { metal } \\
\text { block - } \\
\text { vacuum } \\
\text { bag }\end{array}$ & Autoclave & $\begin{array}{l} \pm 0.004 \text { in. } \\
( \pm 0.102 \mathrm{~mm}) \\
\text { on thickness } \\
\text { mold line as } \\
\text { good as tool }\end{array}$ & $\begin{array}{l}\text { Accurate mold line } \\
\text { tolerance. Excellent } \\
\text { adaptability to compound } \\
\text { contours }\end{array}$ \\
\hline $\begin{array}{l}\text { Plastic } \\
\text { laminate } \\
\text { hat } \\
\text { sections }\end{array}$ & $\begin{array}{l}0.03 \\
\text { Limited } \\
\text { fabricab }\end{array}$ & $\begin{array}{l}b_{\text {by }} 0.762 \\
\text { lity }\end{array}$ & No limit & & $\begin{array}{l}\text { Female } \\
\text { metal } \\
\text { block - } \\
\text { vacuum } \\
\text { bag }\end{array}$ & Autoclave & Same as above & $\begin{array}{l}\text { Difficulties in bonding } \\
\text { for assembly }\end{array}$ \\
\hline
\end{tabular}


TABLE 11d. SUMMARY OF FABRICATION FACTORS - BEAD STIFFENED SKIN PANEL

\begin{tabular}{|c|c|c|c|c|c|c|c|c|}
\hline \multirow[b]{2}{*}{ Item } & \multicolumn{2}{|c|}{$\begin{array}{l}\text { Minimum } \\
\text { gage }\end{array}$} & \multicolumn{2}{|c|}{$\begin{array}{c}\text { Curvature } \\
\text { restrictions }\end{array}$} & \multirow[b]{2}{*}{ Tooling } & \multirow{2}{*}{$\begin{array}{c}\text { Tool heating } \\
\text { method }\end{array}$} & \multirow[b]{2}{*}{ Tolerance } & \multirow[b]{2}{*}{ Remarks } \\
\hline & in. & $\mathrm{cm}$ & in. / in. & $\mathrm{m} / \mathrm{m}$ & & & & \\
\hline $\begin{array}{l}\text { Skin } \\
\text { sheets }\end{array}$ & -- & -- & -- & - & - & -- & -- & $\begin{array}{l}\text { See Table 11a } \\
\text { honeycomb } \\
\text { panel facing } \\
\text { notes apply } \\
\text { here }\end{array}$ \\
\hline $\begin{array}{l}\text { Beaded } \\
\text { titanium } \\
\text { skin }\end{array}$ & $\begin{array}{l}0.020 \\
\text { Limited }\end{array}$ & $\begin{array}{c}0.508 \\
\text { by forming }\end{array}$ & $36 / 3$ & $0.91 / 0.076$ & $\begin{array}{l}\text { Steel } \\
\text { marform } \\
\text { die-stress } \\
\text { relief } \\
\text { fixture }\end{array}$ & $\begin{array}{l}\text { Radiant } \\
\text { heat lamp }\end{array}$ & $\begin{array}{l}0.032 \mathrm{in} . \\
\text { (0.813 mm) } \\
\text { on mold line }\end{array}$ & \\
\hline $\begin{array}{l}\text { Beaded } \\
\text { steel } \\
\text { skin }\end{array}$ & $\begin{array}{l}0.020 \\
\text { Limited }\end{array}$ & $\begin{array}{c}0.508 \\
\text { by forming }\end{array}$ & $36 / 4$ & $0.91 / 0.102$ & $\begin{array}{l}\text { Steel } \\
\text { marform } \\
\text { die }\end{array}$ & $\begin{array}{l}\text { None re- } \\
\text { quired }\end{array}$ & Same as above & $\begin{array}{l}\text { Heat treat in } \\
\text { retort or hy } \\
\text { drogen bell } \\
\text { furnace }\end{array}$ \\
\hline $\begin{array}{l}\text { Beaded } \\
\text { plastic } \\
\text { laminate }\end{array}$ & $\begin{array}{l}0.020 \\
\text { Limited } \\
\text { availabi1 } \\
\text { structur }\end{array}$ & $\begin{array}{l}0.508 \\
\text { by material } \\
\text { lity and } \\
\text { al design }\end{array}$ & No limit & -- & $\begin{array}{l}\text { Male } \\
\text { metal } \\
\text { block - } \\
\text { vacuum } \\
\text { bag }\end{array}$ & Autoclave & $\begin{array}{l} \pm 0.004 \text { in. } \\
( \pm 0.102 \mathrm{~mm}) \\
\text { on thickness } \\
\text { mold line } \\
\text { as good as } \\
\text { tool }\end{array}$ & $\begin{array}{l}\text { Accurate mold } \\
\text { line tolerance. } \\
\text { Excellent } \\
\text { adaptability to } \\
\text { compound con- } \\
\text { tours }\end{array}$ \\
\hline
\end{tabular}


TABLE 11e. SUMMARY OF FABRICATION FACTORS -MACHINED WAFFLE SKIN PANEL

\begin{tabular}{|c|c|c|c|c|c|c|c|c|}
\hline \multirow[b]{2}{*}{ Material } & \multicolumn{2}{|c|}{$\begin{array}{l}\text { Maximum } \\
\text { gage }\end{array}$} & \multicolumn{2}{|c|}{$\begin{array}{l}\text { Curvature } \\
\text { restrictions }\end{array}$} & \multirow[b]{2}{*}{ Tooling } & \multirow{2}{*}{$\begin{array}{c}\text { Tool heating } \\
\text { method }\end{array}$} & \multirow[b]{2}{*}{ Tolerance } & \multirow[b]{2}{*}{ Remarks } \\
\hline & in. & $\mathrm{mm}$ & in. / in. & $\mathrm{m} / \mathrm{m}$ & & & & \\
\hline Titanium & $\begin{array}{l}0.25 \\
\text { Lar } \\
\text { wou } \\
\text { deve }\end{array}$ & $\begin{array}{l}6.35 \\
\text { ages } \\
\text { quire } \\
\text { lent }\end{array}$ & $18 / 3$ & $0.455 / 0.076$ & $\begin{array}{l}\text { Drop } \\
\text { hammer- } \\
\text { vacuum } \\
\text { plate for } \\
\text { machin- } \\
\text { ing }\end{array}$ & $\begin{array}{l}\text { Radiant } \\
\text { heat lamp }\end{array}$ & $\begin{array}{l}0.062 \mathrm{in} . \\
(1.57 \mathrm{~mm}) \\
\text { on mold line }\end{array}$ & $\begin{array}{l}\text { Contour before } \\
\text { machining. } \\
\text { Development of } \\
\text { springback } \\
\text { could cut mold } \\
\text { line tolerance } \\
\text { in half. Machine } \\
\text { or chem-mill } \\
\text { after contouring. } \\
\text { Expensive }\end{array}$ \\
\hline Steel & $\begin{array}{r}0.50 \\
\text { Lar } \\
\text { wou } \\
\text { dev }\end{array}$ & $\begin{array}{l}12.70 \\
\text { ages } \\
\text { quire } \\
\text { gent }\end{array}$ & $18 / 3$ & $0.455 / 0.076$ & $\begin{array}{l}\text { Drop } \\
\text { hammer - } \\
\text { vacuum } \\
\text { plate for } \\
\text { machin- } \\
\text { ing }\end{array}$ & $\begin{array}{l}\text { None re- } \\
\text { quired }\end{array}$ & Same as above & Same as above \\
\hline
\end{tabular}


The use of a heat shield panel concept in lieu of covering an entire vehicle greatly simplifies the inspection and quality control aspects. Radiographic inspection for voids can be readily employed on the plastic laminate substrate/ablator composite. However, the depth of the voids would be difficult to define with current state of the art techniques. Evaluation of the integrity of the honeycomb supporting matrix bond to the substrate panel is somewhat questionable. Low frequency ultrasonics appear promising but need further development. Research is currently being conducted on this problem on related programs. The use of plastic laminates implies the need for additional nondestructive testing of the laminates for voids prior to assembly. In-process controls (material shelf life, autoclave temperatures, curing cycles) must also be maintained.

Prior experience has indicated a problem area related to the warpage of composite panels during and after fabrication post-cure cycles. It is expected this problem will be more pronounced as panel sizes increase and that development work will be required in order to generate a solution. This problem will require resolution by optimizing the sequence of bonding operations and directional properties of the materials to minimize distortion.

\section{Material and Fabrication Cost Comparisons}

Cost comparison data have been generated relative to the candidate heat shield concepts and materials. The information is presented in Tables 12 and 13 .

Table 12 contains the basic material costs for the candidate substrate materials. These costs are based on direct vendor quotations and apply to $500-1 b(226.8-\mathrm{kg})$ lots or greater for the metals and 1000 linear yards $(914.4 \mathrm{~m})$, or greater for the laminating materials unless otherwise noted.

Table 13 defines preliminary estimates of the panel costs per unit area for materials and fabrication. These numbers are based on a model of 10 identical panels of $6 \mathrm{ft}^{2}\left(0.557 \mathrm{~m}^{2}\right)$ area each. The 10identical-panel model was chosen on the assumption that 8 vehicles would be used with a requirement of 2 spare panels. Note that the costs do not include engineering or installation. The costs quoted are for a composite of tooling, fabrication and quality control labor as well as tooling and fabrication materials. Overhead, general and administrative burden and fee are also included. 
TABLE 12. SUBSTRATE PANEL MATERIAL COST SUMMARY

\begin{tabular}{|c|c|c|c|c|c|c|c|c|}
\hline \multirow[b]{2}{*}{ Material } & \multicolumn{2}{|c|}{ Finished thickness } & \multirow[b]{2}{*}{ Sheet size } & \multirow{2}{*}{$\begin{array}{l}\text { Cost } \\
\text { \$/ unit }\end{array}$} & \multicolumn{2}{|c|}{ Costiarea } & \multicolumn{2}{|c|}{ Cost/weight } \\
\hline & in. & $\mathrm{mm}$ & & & $\$ / \mathrm{ft}^{2}$ & $\$ / \mathrm{m}^{2}$ & $\$ / 1 b$ & $\$ / \mathrm{kg}$ \\
\hline $\begin{array}{l}\text { Titanium } \\
(6 A 1-4 V)\end{array}$ & $\begin{array}{l}0.015 \\
0.020 \\
0.030\end{array}$ & $\begin{array}{l}0.381 \\
0.508 \\
0.762\end{array}$ & $\begin{array}{l}36 \times 96 \text { in. } \\
0.914 \times 2.44 \mathrm{~m}\end{array}$ & $\begin{array}{l}306 \\
205 \\
242\end{array}$ & $\begin{array}{r}12.75 \\
8.55 \\
10.01\end{array}$ & $\begin{array}{r}137.24 \\
92.00 \\
107.71\end{array}$ & $\begin{array}{l}36.90 \\
18.50 \\
14.60\end{array}$ & $\begin{array}{l}81.36 \\
40.79 \\
32.19\end{array}$ \\
\hline $\begin{array}{l}\text { Beryllium } \\
\text { (QMV cross } \\
\text { rolled } \\
\text { sheet) }\end{array}$ & $\begin{array}{l}0.01 \\
0.02 \\
0.03\end{array}$ & $\begin{array}{l}0.254 \\
0.508 \\
0.762\end{array}$ & $\begin{array}{l}36 \times 96 \text { in. } \\
0.914 \times 2.44 \mathrm{~m}\end{array}$ & $\begin{array}{l}3308 \\
2734 \\
2978\end{array}$ & $\begin{array}{l}138.00 \\
114.00 \\
124.00\end{array}$ & $\begin{array}{l}1484.88 \\
1226.64 \\
1334.24\end{array}$ & $\begin{array}{r}1430.00 \\
589.00 \\
427.00\end{array}$ & $\begin{array}{r}3153.15 \\
1298.75 \\
941.54\end{array}$ \\
\hline \multirow{2}{*}{$\begin{array}{l}\text { Stainless } \\
\text { steel (17-7 } \\
\text { PH TH 1050) }\end{array}$} & 0.01 & 0.254 & $\begin{array}{l}36 \times 164 \text { in } \\
0.914 \times 4.17 \mathrm{~m}\end{array}$ & 25.70 & 0.63 & 6.78 & 1.50 & 3.31 \\
\hline & $\begin{array}{l}0.02 \\
0.03\end{array}$ & $\begin{array}{l}0.508 \\
0.762\end{array}$ & $\begin{array}{l}44 \times 164 \text { in } \\
1.12 \times 4.17 \mathrm{~m}\end{array}$ & $\begin{array}{l}50.10 \\
70.40\end{array}$ & $\begin{array}{l}1.00 \\
1.41\end{array}$ & $\begin{array}{l}10.76 \\
15.17\end{array}$ & $\begin{array}{l}1.20 \\
1.12\end{array}$ & $\begin{array}{l}2.65 \\
2.47\end{array}$ \\
\hline $\begin{array}{l}\text { Narmeo } 506 \\
\text { prepreg "E" } \\
\text { glass }\end{array}$ & $\begin{array}{l}0.028 \\
0.042\end{array}$ & $\begin{array}{l}0.711 \\
1.067\end{array}$ & $\begin{array}{l}50 \text { in. }(1.27 \mathrm{~m}) \\
\text { wide rolls } \\
1000 \text { yd } 914.4 \mathrm{~m}\end{array}$ & $2420 / \mathrm{rl}$ & $\begin{array}{l}0.45 \\
0.67\end{array}$ & $\begin{array}{l}4.84 \\
7.21\end{array}$ & $\begin{array}{l}1.79 \\
1.79\end{array}$ & $\begin{array}{l}3.95 \\
3.95\end{array}$ \\
\hline $\begin{array}{l}\text { Narmco } \\
\text { Imidite } \\
1850\end{array}$ & $\begin{array}{l}0.02 \\
0.04\end{array}$ & $\begin{array}{l}0.508 \\
1.02\end{array}$ & $\begin{array}{l}18 \text { in. }(0.457 \mathrm{~m}) \\
\text { wide rolis } \\
50 \text { yd } \\
45.7 \mathrm{~m}\end{array}$ & $1250 / \mathrm{rl}$ & $\begin{array}{l}16.65 \\
33.30\end{array}$ & $\begin{array}{l}179.15 \\
358.31\end{array}$ & $\begin{array}{l}93.50 \\
93.50\end{array}$ & $\begin{array}{l}206.17 \\
206.17\end{array}$ \\
\hline
\end{tabular}


TABLE 13. PRELIMINARY COST COMPARISON CHART

\begin{tabular}{|c|c|c|c|c|c|c|}
\hline \multirow[b]{3}{*}{ Face } & \multicolumn{6}{|c|}{ Substrate } \\
\hline & \multicolumn{3}{|c|}{ Honeycomb } & \multicolumn{3}{|c|}{ Skin corrugation } \\
\hline & Plastic & Stainless steel & Titanium & Plastic & Stainless steel & Titanium \\
\hline Core & Plastic & Plastic & Plastic & Plastic & Stainless steel & Titanium \\
\hline \multicolumn{7}{|l|}{$\begin{array}{l}\text { Type of } \\
\text { ablator }\end{array}$} \\
\hline $\begin{array}{l}\text { NASA } 602 \\
\text { elastomer }\end{array}$ & & & & & & \\
\hline$\$$ per sq ft & 631 & 552 & 713 & 1,059 & 913 & 1,601 \\
\hline $\begin{array}{l}\$ \text { per sq } \\
\text { meter }\end{array}$ & 6790 & 5940 & 7672 & 11,395 & 9824 & 17,227 \\
\hline LDNP & & & & & & \\
\hline$\$$ per sq ft & 1,074 & 998 & 1,166 & 1,509 & 1,215 & 1,917 \\
\hline $\begin{array}{l}\$ \text { per sq } \\
\text { meter }\end{array}$ & 11,556 & 10,738 & 12,546 & 16,237 & 13,073 & 20,627 \\
\hline
\end{tabular}

NOTE: Ablator thicknesses are $1 \mathrm{in.}(2.54 \mathrm{~cm})$ for both the NASA 602 and LDNP. 


\section{Concepts Comparison Chart}

In order to compile the results of the screening investigation and present a comparison of the more promising concepts and materials, a concepts comparison chart which assigns a rating to each configuration in the pertinent design categories has been generated. As was done in the initial screening, the system is set up on the basis of a numerical rating for each design category which is then multiplied by a significance factor. 'The rating system is reviewed here for convenience. Note that a finer scale has been used and that a design category has been added for thermal stress compatibility.

\begin{tabular}{|l|c|}
\hline \multicolumn{1}{|c|}{ Category } & $\begin{array}{c}\text { Significance } \\
\text { Factor }\end{array}$ \\
\hline Reliability & 1.0 \\
Structural efficiency (substrate) & 0.90 \\
Thermal stress (ablator and substrate) & 0.80 \\
Fabrication and cost & 0.70 \\
Reparability & 0.50 \\
\hline
\end{tabular}

The basic rating scale is:

\begin{tabular}{|l|l|}
\hline Rating & $\begin{array}{c}\text { Numerical } \\
\text { Value }\end{array}$ \\
\hline Good & 3 \\
Good/fair & $2-1 / 2$ \\
Fair & 2 \\
Fair/poor & $1-1 / 2$ \\
Poor & 1 \\
\hline
\end{tabular}

Since a quantitative comparison of the relative structural efficiencies of the candidate panels is available (Table 10), a more exacting rating system was used here. It consisted of assigning a numerical value of 3 to the lightest panel and a numerical value of 1 to the heaviest panel, which was a titanium double skin corrugation substrate. Intermediate numerical values were selected on the basis of a linear interpolation along an optimum weight versus numerical value plot. A similar system was used in the fabrication and cost category based on the previously discussed cost and fabrication factors.

Since their performance and design aspects are generally comparable, the single and double skin corrugation panels were grouped together. The structural efficiency ratings of the single skin corrugation 
panels were consistently better than those for the double skin-corrugation configurations and were taken as representative of the skincorrugation group.

The concepts comparison chart appears in Table 14. Note that the highest overall rating is for the elastomeric ablator on a plastic laminate honeycomb substrate with a total of $96.2 \%$ of the maximum pos sible points. The elastomeric ablator with a plastic laminate skin corrugation substrate ranks second with a $93.7 \%$ total, but it must be borne in mind that the fabrication and cost rating of 1.7 assigned to this configuration assumes that it is practical to manufacture whereas, in reality, the dimensions associated with the optimum structural weight of this configuration render it impractical from a fabrication point of view.

\section{Selection of Thermostructural Panel Concepts}

Ablator materials. - Investigation of the two candidate ablator materials reveals the NASA 602 elastomeric material to offer advantages over the LDNP when considering reliability, fabrication and cost, reparability, and thermal stress compatibility. However, a heat shield weight advantage is indicated with the use of the LDNP ablator material. In order to evaluate the significance of this weight advantage in more detail, both of the ablator materials were retained for further analysis.

Substrate panel concepts. - Generally speaking, the honeycomb and single skin corrugation concepts are competitive in terms of structural efficiency. In the plastic laminate group, a weight advantage of 0.08 $\mathrm{lb} / \mathrm{ft}^{2}(0.39 \mathrm{~kg} / \mathrm{m})$ is indicated for the single skin corrugation. How ever, the fabrication of structurally optimum single skin corrugation panels of plastic laminate is not practical. For the metallic candidate materials, fabrication problems are more severe for the skin corrugation concepts, and only the beryllium shows a weight advantage over the honeycomb. Accordingly, only the honeycomb concept was selected for further analysis.

Substrate panel materials. - Stainless steel, titanium, beryllium and plastic laminates are discussed in the following paragraphs.

Stainless steel: The use of stainless steel as a substrate panel material has several attractive aspects. From a material performance point of view it offers high reliability. Its application involves relatively low material and fabrication costs and, hence, permits consideration of disposability rather than refurbishment. In addition, studies have shown that it offers good structural efficiency over a wide temperature range. 
TABLE 14. CONCEPTS COMPARISON CHART

\begin{tabular}{|c|c|c|c|c|c|c|c|c|c|}
\hline Concept & Ablator & $\begin{array}{l}\text { Substrate } \\
\text { material }\end{array}$ & $\begin{array}{l}\text { Relia - } \\
\text { bility }\end{array}$ & $\begin{array}{l}\text { Structural } \\
\text { efficiency }\end{array}$ & $\begin{array}{l}\text { Thermal } \\
\text { stress }\end{array}$ & $\begin{array}{l}\text { Fabrication } \\
\text { and cost }\end{array}$ & $\begin{array}{l}\text { Repar- } \\
\text { ability }\end{array}$ & $\begin{array}{l}\text { Total } \\
\text { points }\end{array}$ & $\begin{array}{l}\% \text { of maximum } \\
\text { possible points }\end{array}$ \\
\hline \multirow{8}{*}{$\begin{array}{l}\text { Honeycomb } \\
\text { (fiberglas } \\
\text { core) }\end{array}$} & \multirow[t]{4}{*}{ NASA 602} & Steel & 3 & 2.4 & 2 & 2.0 & 1.5 & 10.9 & 93.2 \\
\hline & & Titanium & 3 & 1.97 & 2 & 1.9 & 1.5 & 10.37 & 88.6 \\
\hline & & Beryllium & 2 & 2.64 & 0.80 & 1.5 & 0.80 & 7.74 & 66.2 \\
\hline & & $\begin{array}{l}\text { Plastic } \\
\text { laminate }\end{array}$ & 3 & 2.26 & 2.4 & 2.1 & 1.5 & 11.26 & 96.2 \\
\hline & \multirow[t]{4}{*}{ LDNP } & Steel & 2.5 & 2.4 & 1.6 & 1.3 & 1.3 & 9.1 & 77.8 \\
\hline & & Titanium & 2.5 & 1.97 & 1.6 & 1.2 & 1.3 & 8.57 & 73.2 \\
\hline & & Beryllium & 1.5 & 2. 64 & 0.80 & 0.80 & 0.50 & 6.24 & 53.3 \\
\hline & & $\begin{array}{l}\text { Plastic } \\
\text { laminate }\end{array}$ & 2.5 & 2. 26 & 2 & 1. 4 & 1. 3 & 9.46 & 80.9 \\
\hline \multirow{8}{*}{$\begin{array}{l}\text { Skin and } \\
\text { corruga- } \\
\text { tion }\end{array}$} & \multirow[t]{4}{*}{ NASA 602} & Steel & 3 & 2.34 & 2 & 1.8 & 1.5 & 10.64 & 90.9 \\
\hline & & Titanium & 3 & 1.54 & 2 & 1.6 & 1. 5 & 9.64 & 82.4 \\
\hline & & Beryllium & 2 & 2.7 & 0.80 & 1.4 & 0.80 & 7.70 & 65.8 \\
\hline & & $\begin{array}{l}\text { Plastic } \\
\text { laminate }\end{array}$ & 3 & 2.36 & 2.4 & 1.7 & 1.5 & 10.96 & 93.7 \\
\hline & \multirow[t]{4}{*}{ LDNP } & Steel & 2.5 & 2.34 & 1.6 & 1.1 & 1.3 & 8. 84 & 75.6 \\
\hline & & Titanium & 2.5 & 1.54 & 1.6 & 0.90 & 1.3 & 7.84 & 67 \\
\hline & & Beryllium & 1.5 & 2.7 & 0.80 & 0.70 & 0.50 & 6.2 & 53 \\
\hline & & $\begin{array}{l}\text { Plastic } \\
\text { laminate }\end{array}$ & 2.5 & 2.36 & 2 & 1.0 & 1.3 & 9.16 & 78.3 \\
\hline
\end{tabular}


The structural efficiency is not compromised by minimum gage limitations. It should be noted, however, that gages less than 0.01 in. $(0.254 \mathrm{~mm})$ are available only in $15.5 \mathrm{in} .(0.394 \mathrm{~m})$ wide coils, the reby requiring splicing in order to attain the required panel sizes. Based on these considerations, stainless steel was retained for further study.

Titanium: Titanium material costs are considerably higher than those for stainless steel. However, if favorable design aspects could be attained from the use of titanium, the material cost would not be prohibitive, though somewhat detrimental to the disposability feature. The use of titanium also introduces fabrication problems in that it must be hot formed. Analysis reveals that the structural efficiency of titanium is severely penalized by a minimum available gage of 0.015 in. $(0.381 \mathrm{~mm})$. The titanium industry is currently developing lower gage capability on a production basis, but no practical results are foreseen in the near future. Without the minimum gage constraint, titanium would be competitive with stainless steel on a structural basis. In view of these limitations, titanium was eliminated from further consideration.

Beryllium: From a structural efficiency standpoint beryllium is outstanding. Based on preliminary analysis, it appears that a beryllium substrate would create serious thermal stress problems. The cost, fabrication and quality control aspects of beryllium are significant drawbacks. It is obvious that the use of a beryllium substrate would have to be based on a requirement for re-use and the attendant refurbishment, inspection and reliability problems. The weight advantage to be gained through the use of beryllium does not outweigh the reliability, cost and fabrication drawbacks associated with this material and further consideration of beryllium was discontinued.

Plastic laminates: In terms of structural efficiency, the laminates are competitive with stajnless steel although somewhat more sensitive to the minimum gage constraint than stainless. The fabrication and cost characteristics are very favorable. The desirable feature of disposability after each mission is also satisfied. The Imidite 1850 laminate shows the need for further development and, although promising, was eliminated from further consideration. Both the phenolic/ glass and phenyl-silane/glass laminates were retained for more detailed investigation.

Adhesive bond. - On the basis of its high temperature capability and, based on its extensive use in the past, high reliability, $\mathrm{HT}-424$ was selected as the adhesive bond system for application to the thermostructural composite. 
Insulation material. - Based on its superior insulation properties and amenability to fabrication over complex surfaces, the insulation material chosen for incorporation into the heat shield system was multilayer microquartz with aluminum foil radiation barriers.

The material and panel concepts selected for detailed investigation are summarized below:

Ablators:

Adhesive bond:

Insulation:

Substrate panel materials:

Substrate panel concept:
NASA 602 elastomer in honeycomb matrix

Low density nylon phenolic

HT -424

Multilayer microquartz with aluminum foil inserts

17-7 PH TH 1050 stainless steel Phenolic resin/E glass laminate Phenyl-silane resin/E glass laminate

Honeycomb panels

\section{ANALYSIS AND OPTIMIZATION OF SELECTED THERMOSTRUCTURAL PANEL CONCEPTS}

In order to develop an insight into the practical engineering implications related to the application of the selected thermostructural panel concepts to a lifting entry vehicle, the NASA Langley HL-10 was considered as the design configuration. Pertinent environmental data have been previously generated under a related task and are defined in ref. 3. Detailed optimization studies were based on the ref. 3 environments.

\section{Panel Size and Orientation Studies}

In establishing the panel overall dimensions (length and width), several factors are significant:

(1) Vehicle size and geometry

(2) Fabricability

(3) Influence of panel attachment method 
(4) Joint material and size limitations

(5) Replacement

(6) Handling requirements

The general philosophy was to make the panels as large as possible within the constraints listed above. A discussion of each factor follows:

Vehicle size and geometry. - The overall vehicle size and contours must be considered regarding their effect on panel size and orientation. Examination of the HL-10 vehicle indicates a basic approach of long, narrow panels running longitudinally along the vehicle with possibly 6 or 8 longitudinal joints and a minimum number of circumferential joints. In areas of relatively sharp curvature, the panel size could be limited by fabrication or purely geometric constraints.

Fabricability. - Panel sizes can be influenced by tooling and other facilities size limitations. For the thin gages expected in the stainless steel substrates $(<0.010 \mathrm{in}$. or $<0.254 \mathrm{~mm})$, the material is available only in 15.5 in. $(0.394 \mathrm{~m})$ wide coils. Obviously to construct a panel of any size it is necessary to splice the narrow strips together. The problem is further compounded by the need to stretch form the sheet to the desired contour. The stretch forming process requires 6 in. $(0.154 \mathrm{~m})$ of material for gripping and an additional $1 \mathrm{in.}(0.0254 \mathrm{~m})$ for trim for a total of 7 in. $(0.178 \mathrm{~m})$ of material on each edge of the sheet. Provided that a practical fabrication technique could be developed, this problem would offer no limit to the panel dimensions. Considering the plastic laminate group, the glass cloth is available on rolls in widths up to 50 in. $(1.27 \mathrm{~m})$, indicating that a panel of up to $4 \mathrm{ft}(1.22 \mathrm{~m})$ in width and any length could be fabricated without need for splicing.

Influence of panel attachment methods. - The two basic modes of panel attachment considered are rigid and flexible. The use of a rigid panel support imposes no constraint on the panel's overall dimensions for panel sizes considered herein. However, the design of a flexible panel support system is predicated in part on the required panel growth the support must permit. Obviously, as the panel size increases the required deflection of the panel support increases. If the panel becomes too large, it can impose a flexibility requirement on the support that makes its design impractical. A stainless steel honeycomb substrate panel requires attacnment with a flexible support system in order to preclude critical thermal stresses and severe structural interaction between the basic vehicle structure and the substrate panel due to differential thermal expansion and load induced deformation. An analysis was developed which relates the thermal deformation of the substrate panel to the flexural strength of a typical support concept and which subsequently defines the maximum permissible panel size in order to preclude a flexural failure of the support. Specifically, the analytical model is defined as follows: 
(1) Stainless steel honeycomb substrate panel is at $800^{\circ} \mathrm{F}\left(700^{\circ} \mathrm{K}\right)$

(2) Phenolic/glass laminate panel support is formed to a channel section for flexibility. (Plastic laminate support selected in order to minimize heat shorts). Support strength is evaluated at $800^{\circ} \mathrm{F}\left(700^{\circ} \mathrm{K}\right)$.

(3) Critical support is located at a corner of the panel and aligned with a principal expansion axis.

(4) Basic vehicle structure is rigid and at room temperature.

(5) The panel is not exposed to any lateral airloads.

The results of the analysis and a sample solution are shown in Figure 43. It is seen that for the case cited, and for practical dimensions (support height $\equiv$ insulation thickness $=1.25$ in. $(31.75 \mathrm{~mm})$, support thickness $=0.04$ in. $(1.02 \mathrm{~mm})$ and a panel aspect ratio of 1.5$)$, the maximum permissible panel dimensions are $20 \times 30$ in. $(0.508 \times 0.762 \mathrm{~m})$. It is apparent that the use of flexible supports can significantly influence the panel size selection.

Joint material limitations. - This problem is analogous to the problem of panel attachment influence on panel size in that the panel size can attain such magnitude as to penalize the design of a sealed joint configuration. The material chosen for the joint sealer has a significant influence on the permissible panel size. The subsection on Joint Design Considerations discusses joint design factors and also develops an analysis for interrelating the panel size with joint material and dimensions. Based on this analysis, it was concluded that for the joint material chosen, the design of the joint had no significant effect on panel size limitations.

Replacement. - Heat shield panels, if damaged to the extent that they cannot be repaired, would require removal and subsequent replacement. From an operational point of view, the advantage here is obviously with a small panel.

Handling requirement. - Handling, shipping, and storage problems increase with panel size. Due to the relative flexibility of large panels, it may be necessary to attach them to a supporting rig prior to mounting on the vehicle. Panel dimensions up to $6 \mathrm{ft}(1.829 \mathrm{~m})$ appear reasonable.

\section{Selection of Heat Shield Panel Sizes and Orientation}

Based on the above considerations, a heat shield panel distribution has been developed for an HL-10 vehicle of $26.67 \mathrm{ft}(8.128 \mathrm{~m})$ reference length. In this case, it was assumed that the substrate panels were plastic laminate honeycomb mounted to the structure on rigid supports. The panel size and orientation would change for a stainless steel substrate 
on flexible supports. The panel breakdown is shown in Figures 44 and 45 and defined quantitatively in Table 15 . It is considered that the fins will be separate removable "panels" in themselves. Likewise, the elevons and nose cap will be separate self-contained removable heat shield systems integral with their related structural components. The elevon cove and aft end of the vehicle could be protected by removable ablative panels but due to the relatively low heat rates in these areas, the panel configurations will probably not conform to those of prime interest in this study. The maximum panel size is $4 \times 6 \mathrm{ft}(1.219 \times 1.829 \mathrm{~m})$ and occurs on the upper surface of the vehicle.

TABLE 15. PROPOSED HEAT SHIELD PANEL BREAKDOWN

\begin{tabular}{|c|c|c|}
\hline Item & $\begin{array}{l}\text { Number } \\
\text { of panels }\end{array}$ & Remarks \\
\hline Upper body & 18 & $\begin{array}{l}\text { Maximum panel size }=4 \times 6 \mathrm{ft} \\
(1.219 \times 1.829 \mathrm{~m})\end{array}$ \\
\hline Leading edge & 10 & \\
\hline Bottom & 11 & \\
\hline Tunnel fairing & 3 & \\
\hline Fins & 3 & $\begin{array}{l}\text { Heat shield and structure removed } \\
\text { as a unit }\end{array}$ \\
\hline Elevons & 2 & $\begin{array}{l}\text { Heat shield and structure removed } \\
\text { as a unit }\end{array}$ \\
\hline Elevon cove & 6 & $\begin{array}{l}\text { Low heating areas. Possibly a } \\
\text { sprayable ablator on fixed vehicle }\end{array}$ \\
\hline Aft end & 3 & $\begin{array}{l}\text { structure or removable panels. } \\
\text { If panels are used, numbers at } \\
\text { left apply. }\end{array}$ \\
\hline Nose cap & 1 & $\begin{array}{l}\text { One piece molded high density } \\
\text { ablator }\end{array}$ \\
\hline
\end{tabular}

Total refurbishable or replaceable heat shield components (including aft end and elevon cove) $=57$

Total number of thermostructural heat shield panels (upper body, leading edge, bottom, and tunnel fairing) $=42$. 


\section{Joint Design Considerations}

The following factors must be considered in examining a panel joint design:
(1) Reliability
(2) Reparability
(3) Effects on panel removability
(4) Compatibility with design environment
(5) Treatment of gaps
(6) Constraints on panel size
(7) Effects on panel orientation

A consideration of prime importance is the treatment of the gaps between the panels. Table 16 compares the advantages and disadvantages of sealed joint gaps versus open joint gaps.

TABLE 16. JOINT TREATMENT CONSIDERATIONS

\begin{tabular}{|c|c|}
\hline Open gaps & Sealed gaps \\
\hline $\begin{array}{l}\text { (1) Loss of surface smoothness } \\
\text { and attendant possibility of local }\end{array}$ & \multirow{6}{*}{$\begin{array}{l}\text { (1) Continuous smooth aerody- } \\
\text { namic surface } \\
\text { (2) Consistent with thermal protec- } \\
\text { tion requirements } \\
\text { (3) Pressure seal } \\
\text { (4) Moisture seal } \\
\text { (5) Must break joint and replace } \\
\text { to remove panel }\end{array}$} \\
\hline $\begin{array}{l}\text { hot spots } \\
\text { (2) Possibility of heat leaks to }\end{array}$ & \\
\hline & \\
\hline $\begin{array}{l}\text { (3) Possibility of pressure build- } \\
\text { up on back of panel }\end{array}$ & \\
\hline $\begin{array}{l}\text { (4) Penetration of moisture during } \\
\text { prelaunch }\end{array}$ & \\
\hline (5) Easier to replace panel & \\
\hline
\end{tabular}

From the above factors, the use of sealed joint gaps is seen to be consistent with the requirement of high reliability; the joint design philosophy was based on the concept of a sealed gap.

The joint material must have sufficient strength and elasticity to accommodate the relative growth or shrinkage between adjacent panels due to temperature changes and structural interaction. The more 
promising materials are RTV 560, RTV 580 and NASA 602 ablator. The RTV materials are characterized by extremely low brittle points (about $-150^{\circ} \mathrm{F}$ or $172^{\circ} \mathrm{K}$ ) and show superior elongation characteristics over the NASA 602 elastomeric ablator in the low temperature range (see Figure 46).

The most severe environmental factor affecting joint strength is orbital cold soak. The joint must grow to accommodate thermal shrinkage of the ablator panel. In addition, in the near vacuum environment during orbit, the pressure structure of the vehicle expands and tends to open the panel joints even further. The effect of pressure growth was evaluated as follows:

Assumptions:

(1) Vehicle pressure structure is 2219-T87 aluminum working at $80 \% \sigma_{\text {ty }}$

$$
\begin{aligned}
& \sigma_{\mathrm{ty}}=50,000 \mathrm{psi}\left(344.7 \frac{\mathrm{MN}}{\mathrm{m}^{2}}\right) \\
& \mathrm{E}=10.5 \times 10^{6} \mathrm{psi}\left(72.39 \frac{\mathrm{GN}}{\mathrm{m}^{2}}\right) \\
& \epsilon=\frac{50,000 \times 0.80}{10.5 \times 10^{6}}=0.0038
\end{aligned}
$$

(2) $\mathrm{X}_{\mathrm{P}}=$ periphery of vehicle section (for analysis of longitudinal joints)

$\mathrm{X}_{1} \quad=$ length of vehicle (for analysis of peripheral joints)

$\mathrm{N}_{\mathrm{J}_{\mathrm{L}}}=$ number of longitudinal joints

$\mathrm{N}_{\mathrm{J}_{\mathrm{P}}}=$ number of peripheral joints

$\epsilon_{\mathrm{J}}=$ strain in joint material

$\mathrm{t}_{\mathrm{J}}=$ joint gap width 


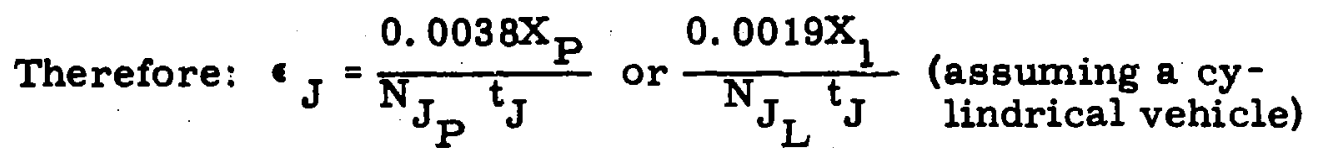

The strain induced in the joint material by thermal deformation is defined by:

$$
\mathrm{J}=e_{\mathrm{p}}\left[-\frac{\mathrm{d}}{\mathrm{t}_{\mathrm{J}}}+\mu_{\mathrm{J}}\right]-\frac{\alpha_{\mathrm{J}} \Delta \mathrm{T}_{\mathrm{J}}}{1-\mu_{\mathrm{J}}}
$$

where:

$$
\begin{aligned}
& d_{L} \text { or } d_{P}=\begin{array}{l}
\text { panel side dimension (longitudinal } d \text { for analysis of } \\
\text { peripheral joints and peripheral } d \text { for analysis of } \\
\text { longitudinal joints) }
\end{array} \\
& \quad=\text { panel strain (- } \epsilon_{p} \text { for shrinkage) } \\
& \quad=\text { Poisson's ratio of joint material } \\
& \mu_{J} \quad=\text { coefficient of thermal expansion for joint material } \\
& \alpha_{J} \quad=\text { temperature change of joint material }\left(-\Delta T_{J}\right. \text { for cold soak) } \\
& \Delta T_{J} \quad
\end{aligned}
$$

Combining the thermal and structural interaction strains yields:

$$
\epsilon_{J}=c_{p}\left[-\frac{d_{P}}{t_{J}}+\mu_{J}\right]-\frac{\alpha_{J} \Delta T_{J}}{1-\mu_{J}}+\frac{0.0038 X_{P}}{N_{J_{P}} t_{J}} \quad \text { (for longi - } \text { tudinal joints) }
$$

A similar equation applies for the analysis of peripheral joints. These equations can be used to establish the constraints imposed by the joint material and gap width on the panel size. Since the ablators have much higher expansion coefficients than the substrate panel materials, a limit condition can be established if we say the ablator thermal contraction is unrestrained. For an orbital cold soak condition with the ablator at $-71^{\circ} \mathrm{F}\left(216^{\circ} \mathrm{K}\right)$, as defined in ref. 3 , an analysis was conducted based on the following analytical model (based on panel sizes and orientations previously defined): 
(1) $\mathrm{X}_{\mathrm{P}}=308 \mathrm{in} .(7.823 \mathrm{~m})$ at station 152

$$
\begin{aligned}
& \mathrm{x}_{1}=320 \mathrm{in} .(8.128 \mathrm{~m}) \\
& \mathrm{N}_{J_{P}}=8 \\
& \mathrm{~N}_{J_{L}}=5 \\
& d_{P}=48 \mathrm{in} \cdot(1.219 \mathrm{~m}) \\
& d_{L}=72 \mathrm{in} .(1.829 \mathrm{~m})
\end{aligned}
$$

(2) RTV 560 ultimate strain $=\epsilon_{\mathrm{J}}=128 \%$

$$
\begin{aligned}
& \mu_{\mathrm{J}}=0.40 \\
& \alpha_{\mathrm{J}} \cong 100 \times 10^{-6} \mathrm{in.} / \mathrm{in} .-^{\circ} \mathrm{F}\left(180 \times 10^{-6} \mathrm{~cm} / \mathrm{cm}^{-\circ} \mathrm{K}\right)
\end{aligned}
$$

(3) NASA $602 \epsilon_{p}=-0.00975$

$$
\text { LDNP } \epsilon_{p}=-0.00339
$$

The intent of the analysis was to define the joint gap dimensions required for the panel sizes and orientations selected and hence determine if the required gap sizes were prohibitive (i.e., does joint design constrain panel size below the dimensions selected?). The calculated required joint gap dimensions are shown in Table 17 . It should be noted that the se are minimum permissible values for the panel considered. A smaller joint gap would result in failure of the joint material.

TABLE 17. MINIMUM JOINT GAP SIZES FOR A 4 X 6 FT $(1.219 \times 1.829 \mathrm{~m})$ PANEL (OR BITAL COLD SOAK)

\begin{tabular}{|c|c|c|c|c|}
\hline \multirow{2}{*}{ Ablator Material } & \multicolumn{2}{|c|}{$\begin{array}{c}\text { Peripheral } \\
\text { joint gap }\end{array}$} & \multicolumn{2}{c|}{$\begin{array}{c}\text { Longitudinal } \\
\text { joint gap }\end{array}$} \\
\cline { 2 - 5 } & in. & $\mathrm{mm}$ & in. & $\mathrm{mm}$ \\
\hline NASA 602 & 0.655 & 16.6 & 0.488 & 12.39 \\
LDNP & 0.291 & 7.39 & 0.246 & 6.25 \\
\hline
\end{tabular}

The minimum permissible gap size decreases with decreasing panel sizes. Elevated temperature conditions should be less critical in terms of joint strength since the joint material is compressed. However, the elevated temperature case could impose a panel size constraint based on the compressibility limit of the joint material. Insufficient material performance data are available for an analytical treatment of this problem. 
The calculated joint gap sizes appear reasonable (approximately $1 \%$ of panel dimension) for the extreme case considered. It may be concluded based on this analysis that for a joint material of RTV 560 silicon elastomer, the interrelationship between joint dimension and panel size is not critical. It should be noted, however, that the analysis does not consider the strength of the bond between the joint sealer and the ablative material. It would be necessary to conduct an element test program to establish the integrity of the joint design and verify the analysis.

Table 18 presents a group of panel joint concepts and lists their relative advantages and disadvantages. From the standpoint of design simplicity and overall functional capability, configuration $\mathbf{C}$ appears most promising.

TABLE 18. JOINT DESIGN CONCEPTS

\begin{tabular}{|c|c|c|}
\hline Configuration & Advantages & Disadvantages \\
\hline A & $\begin{array}{l}\text { 1. Long heat flow } \\
\text { path } \\
2 \text {. Mechanical inter- } \\
\text { lock } \\
3 \text {. Individual panels } \\
\text { can be removed } \\
4 \text {. Large surface } \\
\text { contact with ablator } \\
5 \text {. Easy to break } \\
\text { joint }\end{array}$ & $\begin{array}{l}\text { 1. Requires skewed } \\
\text { edges on all ablator } \\
\text { panels } \\
\text { 2. Edges are suscep- } \\
\text { tible to damage }\end{array}$ \\
\hline B & $\begin{array}{l}\text { 1. Long heat flow } \\
\text { path } \\
2 \text {. Mechanical inter- } \\
\text { lock } \\
3 \text {. Large surface } \\
\text { contact with ablator }\end{array}$ & $\begin{array}{l}\text { 1. Requires intricate } \\
\text { shaping of edge mem- } \\
\text { bers } \\
2 . \text { Individual panels } \\
\text { cannot be removed } \\
\text { 3. Edges are suscep- } \\
\text { tible to damage } \\
4 \text {. Difficult to break } \\
\text { joint for panel removal }\end{array}$ \\
\hline & $\begin{array}{l}\text { 1. Mechanical inter- } \\
\text { lock } \\
2 \text {. Individual panels } \\
\text { can be removed } \\
\text { 3. Easy to break } \\
\text { joint for panel re- } \\
\text { moval }\end{array}$ & $\begin{array}{l}\text { 1. Short heat flow } \\
\text { path } \\
\text { 2. Small surface } \\
\text { contact with ablator }\end{array}$ \\
\hline
\end{tabular}


TABLE 18. concluded

\begin{tabular}{|c|c|c|}
\hline Configuration & Advantages & Disadvantages \\
\hline D & $\begin{array}{l}\text { 1. Mechanical inter- } \\
\text { lock } \\
\text { 2. Long heat flow } \\
\text { path } \\
\text { 3. Large surface } \\
\text { contact with ablator }\end{array}$ & $\begin{array}{l}\text { 1. Individual panels } \\
\text { cannot be removed } \\
2 . \text { Requires intricate } \\
\text { shaping of edge mem- } \\
\text { bers } \\
3 \text {. Edges are ex- } \\
\text { tremely susceptible } \\
\text { to damage } \\
\text { 4. Difficult to break } \\
\text { joint for panel re- } \\
\text { moval }\end{array}$ \\
\hline$\xi$ & $\begin{array}{l}\text { 1. Mechanical inter- } \\
\text { lock } \\
2 \text {. Individual panels } \\
\text { can be removed } \\
\text { 3. Large surface } \\
\text { contact with ablator }\end{array}$ & $\begin{array}{l}\text { 1. Short heat flow } \\
\text { path } \\
2 . \text { Although joint is } \\
\text { easy to break for } \\
\text { panel removal, it is } \\
\text { extremely difficult to } \\
\text { clean out and replace } \\
3 \text {. Requires intricate } \\
\text { shaping of panel edges } \\
4 \text {. Panel edges are } \\
\text { extremely susceptible } \\
\text { to damage }\end{array}$ \\
\hline
\end{tabular}

Substrate Panel/Support Interaction and Flexibility Requirements

Panel supports should be located in such a manner as to minimize moments and deflections in the substrate panel. A comparison of the problems associated with support spacing studies is outlined in Table 19. 
TABLE 19. EFFECTS OF SUPPORT SPACING

\begin{tabular}{|c|c|c|}
\hline & Large support spacing & Small support spacing \\
\hline (1) & $\begin{array}{l}\text { Panel point loadings increase } \\
\text { with possible need for local } \\
\text { beefup of panel }\end{array}$ & $\begin{array}{l}\text { (1) Panel point loadings decrease; } \\
\text { more nearly uniform intro- } \\
\text { duction of load into panel re- } \\
\text { sults }\end{array}$ \\
\hline (2) & $\begin{array}{l}\text { Panel moments and deflec- } \\
\text { tions increase }\end{array}$ & $\begin{array}{l}\text { (2) Panel moments and deflec- } \\
\text { tions decrease }\end{array}$ \\
\hline (3) & Reliability is decreased & Reliability is increased \\
\hline (4) & $\begin{array}{l}\text { Panel and support weights } \\
\text { increase }\end{array}$ & $\begin{array}{l}\text { Panel and support weights } \\
\text { decrease }\end{array}$ \\
\hline (5) & $\begin{array}{l}\text { There are fewer interface } \\
\text { points with pressure struc- } \\
\text { ture and substrate, there- } \\
\text { fore, fewer matching } \\
\text { tolerance problems }\end{array}$ & $\begin{array}{l}\text { (5) Matching problem at pres - } \\
\text { sure shell/substrate inter- } \\
\text { face increases } \\
\text { (6) Heat short problem increases }\end{array}$ \\
\hline (6) & $\begin{array}{l}\text { Heat short problem is } \\
\text { minimized } \\
\text { Advantages to turnaround } \\
\text { and refurbishabllity result }\end{array}$ & $\begin{array}{l}\text { Increased turnaround time } \\
\text { and refurbishability problems } \\
\text { result }\end{array}$ \\
\hline
\end{tabular}

Panel support concepts for all configurations must have load carrying capability between the panel and the pressure structure along the panel's principal load axis. The supports may be omnidirectionally rigid or provide for thermal expansion of the panel while providing load capability as required.

The low elastic moduli that are characteristic of the plastic laminates indicate that the use of omnidirectionally rigid panel supports is possible without creating severe thermal stresses and critical interaction loads between the substrate panel and vehicle structure. The high interaction loads and thermal stresses associated with a rigidly supported stainless steel substrate panel indicate the need for a flexible support system. An analysis evaluating the effect of omnidirectionally rigid supports on the panel thermal stresses and interaction loads was conducted based on the following assumptions.

(1) Thermoelastic behavior

(2) Panel is stable

(3) Thermal expansion is completely restrained.

Figures 47 and 48 compare the substrate panel thermal and allowable material stresses for both the plastic laminates and stainless steel 
substrates. Both materials generate thermal stresses below the critical value up to the limiting substrate panel design temperature of $800^{\circ} \mathrm{F}$ $\left(700^{\circ} \mathrm{K}\right)$. The stainless steel appears more marginal than the plastic laminates, generating $83 \%$ of the allowable stress at $800^{\circ} \mathrm{F}\left(700^{\circ} \mathrm{K}\right)$. Further, the curve indicates a thermal stress failure at $875^{\circ} \mathrm{F}\left(742^{\circ} \mathrm{K}\right)$ for the stainless steel substrate. A comparison of the interaction loads generated between the substrate panel and vehicle structure was developed. Based on a structural thickness of 0.01 in. $(0.254 \mathrm{~mm})$ for stainless steel [two $0.005 \mathrm{in.}(0.127 \mathrm{~mm})$ panel faces], and 0.04 in. $(1.016 \mathrm{~mm})$ for the plastic laminates $[$ two $0.02 \mathrm{in} .(0.508 \mathrm{~mm})$ panel faces] the interaction loads developed at each support point (assuming a support spacing of $10 \mathrm{in}$. or $0.254 \mathrm{~m}$ ) are shown in Table 20 .

TABLE 20. SUBSTRATE PANEL/VEHICLE STRUCTURE INTERACTION LOADS WITH A RIGID SUPPORT SYSTEM

\begin{tabular}{|l|c|c|}
\hline Substrate panel material & \multicolumn{2}{|c|}{$\begin{array}{c}\text { Maximum } \\
\text { interaction load from R. T. to } 800^{\circ} \mathrm{F} \\
\left(700^{\circ} \mathrm{K}\right)\end{array}$} \\
\hline Stainless steel & $1 \mathrm{~b}$ & $\mathrm{~kg}$ \\
Plastic laminate & 11,100 & 5040 \\
\hline
\end{tabular}

Based on the analysis to determine interaction loads, a problem is indicated for both plastic laminate and stainless steel. However, consideration of the tolerances required in such a system in order to meet assembly requirements and relief due to panel bowing will reduce the se values significantly. The subject of panel supports is treated in more detail in ref. 14.

\section{Heat Shield Sizing and Thermal Analysis}

Environmental definition and thermal analysis criteria, -Thermal analyses of both the NASA 602 and LDNP ablator materials were conducted on the basis of application to a NASA Langley HL-10 vehicle of $26.67 \mathrm{ft}(8.128 \mathrm{~m})$ reference lenglh. Reference 3 defines the flight mission profiles upon which the heat shield analysis is based. The entry flight trajectories include a nominal, overshoot, and undershoot heating environment. Subsequent to developing the heat shield thickness requirements, compatibility of the design was checked against the ascent, abort and orbital environments defined in ref. 3.

In order to generate ablator and insulation thickness design curves, an analytical model and a set of thermal design criteria were established and are defined below: 
Analytical model: The analysis was based on one dimensional heat flow through a representative section of a thermostructural heat shield panel. For convenience of definition, the heat shield model is broken down into its separate components:

(1) Ablator: Variable thickness to suit design constraints. Both NASA 602 and LDNP were considered.

(2) Substrate Panel: Phenolic/glass honeycomb panel with 0.02 in. $(0.508 \mathrm{~mm})$ faces, $5.5 \mathrm{lb} / \mathrm{ft}^{3}\left(88.11 \mathrm{~kg} / \mathrm{m}^{3}\right)$ core. Total panel depth $=0.375$ in. $(9.53 \mathrm{~mm})$.

(3) Insulation: Multi-layer microquartz with aluminum foil inserts. $6.64 \mathrm{lb} / \mathrm{ft}^{3}\left(106.37 \mathrm{~kg} / \mathrm{m}^{3}\right)$ density. Variable thickness to suit design constraints.

(4) Vehicle Structure: Aluminum, $0.050 \mathrm{in.}(1.27 \mathrm{~mm})$ thickness. For purposes of design curve development, no heat losses from the aluminum structure were considered.

Thermal design criteria: The thermal design criteria are as follows.

(1) Safety Factors: For development of the heat shield design curves, no factors were applied to heating rates or thicknesses.

(2) Temperature Constraints: The maximum permissible bond line temperature is $800^{\circ} \mathrm{F}\left(700^{\circ} \mathrm{K}\right)$. The vehicle structare maximum temperature is $150^{\circ} \mathrm{F}\left(339^{\circ} \mathrm{K}\right)$ at or before touchdown. After touchdown the temperature is not permitted to exceed $400^{\circ} \mathrm{F}\left(478^{\circ} \mathrm{K}\right)$.

(3) Heat Shield Temperature Distribution at De-Orbit: Based on the orbital thermal environment defined in ref. 3 , it is possible for the surface of the ablator to attain a maximum temperature of $282^{\circ} \mathrm{F}\left(412^{\circ} \mathrm{K}\right)$. For purposes of heat shield design curve development, the temperature distribution in the heat shield at the initiation of re-entry consists of $282^{\circ} \mathrm{F}$ $\left(412^{\circ} \mathrm{K}\right)$ through the depth of the ablator to the ablator/substrate interface. From this point the temperature decreases linearly through the substrate panel and insulation to a value of $70^{\circ} \mathrm{F}\left(294^{\circ} \mathrm{K}\right)$ at the aluminum structure.

(4) Heat Shield Temperature Distribution at Launch: The temperature distribution in the heat shield at launch is taken to be $125^{\circ} \mathrm{F}\left(325^{\circ} \mathrm{K}\right)$ constant through the ablator depth to the 
ablator/substrate panel interface. From this point the temperature decreases linearly through the substrate panel and insulation to a value of $70^{\circ} \mathrm{F}\left(294^{\circ} \mathrm{K}\right)$ at the aluminum structure.

Development of heat shield design curves, - Data upon which the heat shield design curves are based were calculated on the Martin T-CAP III digital program. Results are contained in Figures 49 through 52. Ablator and insulation thickness requirements are shown as a function of percentage of stagnation point heating, thereby permitting application of these data to any point on the vehicle. Note that design curves are presented for both the nominal (total cold wall heat input at stagnation point $=110,500 \mathrm{Btu} / \mathrm{ft}^{2}$ or $1.255 \mathrm{GJ} / \mathrm{m}^{2}$ for $\left(\frac{\mathrm{L}}{\mathrm{D}}\right)_{\max }$ ) and the overshoot $\left(69,500 \mathrm{Btu} / \mathrm{ft}^{2}\right.$ or $789.29 \mathrm{MJ} / \mathrm{m}^{2}$ for $\left(\mathrm{C}_{\mathrm{L}}\right) \underset{\max }{\text { ) } \mathrm{re}^{-}}$ entry cases and for both of the candidate ablator materials. Insulation requirements are defined for maximum structure temperatures at or before touchdown of $200^{\circ} \mathrm{F}$ and $250^{\circ} \mathrm{F}\left(376^{\circ} \mathrm{K}\right.$ and $\left.394^{\circ} \mathrm{K}\right)$ in addition to the $150^{\circ} \mathrm{F}\left(339^{\circ} \mathrm{K}\right)$ requirement. For this temperature and insulation thickness range, the ablator thickness curve is unaffected by variations in structural temperature levels due to the "infinite slab" effect of the insulation. In essence, for the range considered here, the ablator and insulation thickness requirements are decoupled in the sense that the maximum permissible bondline temperature dictates the ablator thickness, and the insulation thickness is dependent upon the permissible structural temperature. The design curves, however, are based on an analysis in which the interaction between ablator and insulation thicknesses was considered.

Interpretation of heat shield design curves.- The ablator thickness requirements as a function of absolute values of total cold wall stagnation point heat input are plotted in Figure 53 for both the nominal and overshoot trajectories and both the NASA 602 and LDNP ablators. Note the similarity between the curves for the same material when exposed to different re-entry environments. Note also that the LDNP thickness curve crosses over the NASA 602 curve at the higher total heat levels for the nominal trajectory. This is a manifestation of the higher surface recession rates of the LDNP in this heating rate range.

Figures 54 and 55 define the ablator and insulation weight requirements for the cases considered.

On the basis of the heating rate distributions defined in ref, 3 , the heat shield design curve data was applied to the HL-10 vehicle. It was found that the overshoot trajectory dictated the insulation thicknesses on all portions of the vehicle. The ablator thickness requirements were found to be dependent upon either the nominal or overshoot trajectory depending on the vehicle location under consideration. Figures 56 and 57 define the critical trajectory boundaries for establishing ablator 
thicknesses. The higher angle of attack associated with the overshoot re-entry results in higher $\dot{\mathrm{q}}_{L} / \dot{\mathrm{q}}_{\text {stag. }}$ ratios over the lower portion of the vehicle. Comparing the distributions between the LDNP and NASA 602 heat shields shows the effect of material on the critical trajectory boundaries. In this case the significant factor is the greater surface recession sensitivity of the LDNP.

By designing the heat shield as a composite of more than one critical flight trajectory, an interesting situation is created. For example, for a point on the vehicle where the ablator thickness is dictated by the nominal trajectory, and (as at all points in this case) the insulation thickness is based on the overshoot trajectory, a nominal re-entry flight would result in attaining the limiting bondline temperature, but the peak structure temperature would fall below the prescribed limit. For the same vehicle location exposed to an overshoot re-entry, both the peak bondline and structure temperatures would fall below their maximum limit. Obviously, the use of a design philosophy such as this will significantly influence the establishment of heat shield safety factor criteria.

An estimate was made of the critical $\dot{\mathrm{q}}_{\mathrm{L}} / \dot{\mathrm{q}}_{\text {stag. }}$ ratio below which no ablative heat shield would be required. The analysis was based on determining the heating rate associated with a radiation equilibrium temperature of $800^{\circ} \mathrm{F}\left(700^{\circ} \mathrm{K}\right)$ and subsequently determining what percentage of the maximum stagnation point heating rate for the nominal, overshoot, and undershoot trajectories was required in order to attain this value. The resulting ratio is the critical $\dot{\mathrm{q}}_{\mathrm{L}} / \dot{\mathrm{q}}_{\text {stag. }}$ value. Table 21 presents the critical ratios.

TABLE 21. CRITICAL $\dot{\mathrm{q}}_{L} / \dot{\mathrm{q}}_{\text {stag. }}$ RATIOS

\begin{tabular}{|l|c|}
\hline Trajectory & Critical $\dot{\mathrm{q}}_{\mathrm{L}} / \dot{\mathrm{q}}_{\text {stag. }}$ \\
\hline Nominal & 0.01 \\
Overshoot & 0.0164 \\
Undershoot & 0.0035 \\
\hline
\end{tabular}

Since the heat shield must be designed for the entire re-entry flight corridor, the critical ratio becomes 0.0035 , which indicates that for heat shield systems incorporating bonded substrate panels (temperature limit $800^{\circ} \mathrm{F}$ or $700^{\circ} \mathrm{K}$ ) such as those considered in this study, ablative protection will be required at all vehicle locations. 
Compatibility of heat shield with mission thermal design envelope. The heat shield design must be capable of exposure to the thermal environment associated with all phases of its operational mission envelope while maintaining its structural integrity and retaining critical areas within their prescribed temperature constraints.

A representative heat shield point was subjected to the undershoot trajectory heating environment in order to determine the effect on critical heat shield temperatures. The point chosen was at vehicle station 19.2 (see Figure 44) since this represents the most severe thermal environment to which any of the panel concepts would be subjected. The maximum $\dot{\mathrm{q}}_{\mathrm{L}} / \dot{\mathrm{q}}_{\text {stag. }}$ at this station is 0.565 . Since the undershoot re-entry is characterized by high heating rates and short exposure times, the LDNP ablator was analyzed because of its predicted higher surface recession rates and, hence, greater sensitivity to the high heating rates associated with the undershoot re-entry. For the point considered, the LDNP thickness is dictated by the nominal re-entry as $1.465 \mathrm{in} .(37.21 \mathrm{~mm})$ and the insulation thickness as required by the overshoot re-entry is $1.34 \mathrm{in} .(34.04 \mathrm{~mm})$. Note that for the overshoot re-entry the $\dot{\mathrm{q}}_{\mathrm{L}} / \dot{\mathrm{q}}_{\text {stag. }}$ at the design point is 0.70 , whereas for both the undershoot and nominal, the ratio is 0.565 . By use of the same analytical model as for the design curves and the previously defined thicknesses, the heat shield configuration was exposed to the undershoot re-entry thermal environment. The resulting maximum bondline and structure temperatures are shown in Table 22 .

TABLE 22. COMPARISON OF CRITICAL HEAT SHIELD TEMPERATURES FOR UNDERSHOOT RE-ENTRY

\begin{tabular}{|l|c|c|c|c|}
\hline \multirow{2}{*}{ Item } & \multicolumn{2}{|c|}{ Upper limit } & \multicolumn{3}{|c|}{ Actual value } \\
\cline { 2 - 5 } & ${ }^{\circ} \mathrm{F}$ & ${ }^{\circ} \mathrm{K}$ & ${ }^{\circ} \mathrm{F}$ & ${ }^{\circ} \mathrm{K}$ \\
\hline Maximum bondline temperature & 800 & 700 & 435 & 497 \\
Maximum structure temperature & 150 & 339 & 90 & 306 \\
\hline
\end{tabular}

The maximum calculated surface recession is 0.58 in. $(14.73 \mathrm{~mm})$. Based on this analysis, the undershoot trajectory does not appear critical for heat shield design.

Reference 3 defines a maximum heating rate abort trajectory during vehicle boost. Examination of the significant heating parameters reveals the thermal environment to be less critical than that for the undershoot re-entry case. A comparison of the significant thermal environment parameters associated with the undershoot and maximum heating rate abort cases is shown in Table 23. 
TABLE 23. COMPARISON OF UNDERSHOOT AND MAXIMUM HEATING RATE ABORT TRAJECTORIES

\begin{tabular}{|l|c|c|c|c|c|}
\hline \multirow{2}{*}{ Trajectory } & \multicolumn{2}{|c|}{$\begin{array}{c}\text { Peak stagnation } \\
\text { heating rate }\end{array}$} & \multicolumn{2}{c|}{$\begin{array}{c}\text { Maximum } \\
\text { stagnation } \\
\text { heat input }\end{array}$} & $\begin{array}{c}\text { Time from } \\
\text { start of } \\
\text { heating } \\
\text { to touchdown, } \\
\text { sec }\end{array}$ \\
\cline { 2 - 6 } & $\mathrm{Btu} / \mathrm{ft}^{2}-\mathrm{sec}$ & $\mathrm{Mw} / \mathrm{m}^{2}$ & $\mathrm{Btu} / \mathrm{ft}^{2}$ & $\mathrm{MJ} / \mathrm{m}^{2}$ & $\begin{array}{c}\text { touch } \\
\begin{array}{l}\text { Undershoot } \\
\text { re-entry }\end{array}\end{array}$ \\
$\begin{array}{l}\text { Maximum } \\
\text { heating } \\
\text { rate abort }\end{array}$ & 104 & 3.087 & 51,500 & 584.9 & 1340 \\
\hline
\end{tabular}

During boost to orbit, the unshrouded vehicle experiences heating of a moderate nature. Of prime concern here is that no degradation of the ablative heat shield is experienced during ascent to the extent that the heat shield's ability to sustain re-entry heating is compromised. An analysis of a typical heat shield was conducted based on the ascent trajectory defined in reference 3 . In order to define the worst pos sible case, the stagnation point thermal environment was imposed on a NASA 602 ablator of 1.63 in. $(41.40 \mathrm{~mm})$ thickness. The insulation thickness was 1.29 in. $(32.77 \mathrm{~mm})$. T-CAP III analysis revealed that the ascent heating environment pyrolyzes $2.3 \%$ by weight of the ablator. Since this degradation occurred at the stagnation point, the problem of ascent heating degrading the heat shield is, in this case, not critical.

Another potential problem associated with ascent heating is that elevated temperatures cause the loss of ablator strain capability which could result in subsequent failure during orbital cold soak.

Insulation optimization studies. - Tests were conducted on the Martin guarded hot plate facility in order to verify the microquartz insulation effective thermal conductivity values used in the analysis for required heat shield thicknesses. The test specimen consisted of 7 layers of microquartz fiber mats with 0.005 in. $(0.127 \mathrm{~mm})$ alumi num foils between each layer. An additional foil layer was added at the top and bottom of the stack. The testing procedure conformed to ASTM-C-177-63. Tests were run at a number of atmospheric pressures and two temperature levels. In one case the outside face of the specimen was held at $800^{\circ} \mathrm{F}\left(700^{\circ} \mathrm{K}\right)$ and the inside face at $80^{\circ} \mathrm{F}$ $\left(300^{\circ} \mathrm{K}\right)$. In the second case, the outside specimen face was held at $300^{\circ} \mathrm{F}\left(422^{\circ} \mathrm{K}\right)$ and the inside face at $80^{\circ} \mathrm{F}\left(300^{\circ} \mathrm{K}\right)$. Resultant effective thermal conductivity values were taken to be at the average temperature of $440^{\circ} \mathrm{F}\left(500^{\circ} \mathrm{K}\right)$ for the first case and $190^{\circ} \mathrm{F}\left(361^{\circ} \mathrm{K}\right)$ 
for the second. Test results are shown in Figure 58. For comparative purposes the values used for the heat shield design curve analysis are also presented. Note that the value used for analysis is based on a pressure of $0.0105 \mathrm{~atm}$. This was taken to be a representative value over the entire re-entry run and is equivalent to the ambient pressure at $100,000-\mathrm{ft}(304.8 \mathrm{~km})$ altitude. The test values are significantly greater than those used for design. Reference 5 indicates that the thermal conductivity of low density fiber insulations is extremely sensitive to mechanical pressure. A mechanical pressure existed on the test specimen due to the installation setup and was calculated to be $0.052 \mathrm{psi}\left(358.53 \mathrm{~N} / \mathrm{m}^{2}\right)$. Additional tests were run on the same specimen with the foil radiation barriers removed. The results are also shown in Figure 58 and reveal that for the temperature range of interest in this study $\left(70^{\circ}\right.$ to $800^{\circ} \mathrm{F}$ or $294^{\circ}$ to $\left.700^{\circ} \mathrm{K}\right)$, the aluminum foil inserts can be removed from the insulation system with virtually no increase in the thermal conductivity. Although at temperatures of $800^{\circ} \mathrm{F}\left(700^{\circ} \mathrm{K}\right)$ radiant heat transfer would normally contribute some amount to the effective thermal conductivity, it is indicated that, in this case, the microquartz remains opaque to the wavelengths associated with radiant heat transfer at these temperature levels. Figure 59 presents the atmospheric pressure dependence of the microquartz insulation thermal conductivity. The apparent sensitivity to atmospheric pressure indicates the need to establish a practical pressure criteria on which to base the thermal conductivity for detail heat shield sizing analysis (or buy a weight penalty by assuming 1 -atm values).

A design concept was investigated whereby the thermal insulation and panel support functions were integrated into a single component. The integral insulation/support system consists of a composite of alternating layers of phenolic/glass flexible honeycomb core and aluminum foil (see Figure 60). The use of such a system would provide continuous support to the heat shield substrate panel under an external airload. A pattern of post type supports would penetrate the insulation/ support system at discrete locations in order to tie the ablator/substrate panel to the vehicle structure. However, since the post supports would be relieved of the critical requirement of carrying panel overpressures to the vehicle structure, the amount of post supports could be greatly decreased since their spacing would now be dictated by the less critical requirements imposed by negative panel pressures and flutter criteria. The continuous support concept also minimizes the introduction of relatively high local point loadings into the vehicle structure, thereby reducing the need for the addition of structural "hard points" to the basic structural shell and the attendant weight penalty. Since bonding the flexible core/foil elements together to form a composite would destroy the ability of the foils to act as radiation barriers, an alternate approach to assembling the system was selected; it con- 
sists of stacking the individual components to the desired depth and enclosing the composite aystem in a coarse mesh glass cloth bag prior to installation on the vehicle.

Analysis of the concept was conducted in order to establish the optimum number of foils. Results are shown in Figure 61 for the case of 1-atm pressure (heat conducted through air) and a complete vacuum. Note that the foil gage is assumed to be 0.005 in. $(0.127 \mathrm{~mm})$. Thinner foils could be used but the danger of tearing the foils on the sharp honeycomb cell edges would be increased. The basic honeycomb core density is $2.5 \mathrm{lb} / \mathrm{ft}^{3}\left(40.05 \mathrm{~kg} / \mathrm{m}^{3}\right)$.

The calculated density of a $1.25 \mathrm{in} .(31.75 \mathrm{~mm})$ thick honeycomb/ foil composite with five foil layers is $5.96 \mathrm{lb} / \mathrm{ft}^{3}\left(95.48 \mathrm{~kg} / \mathrm{m}^{3}\right)$ which is competitive with the density of the multilayer microquartz (with no foils) at $6.21 \mathrm{lb} / \mathrm{ft}^{3}\left(99.48 \mathrm{~kg} / \mathrm{m}^{3}\right)$. Considering the 1 -atm case, the composite optimizes at three foil layers for a net density of $4.58 \mathrm{lb} /$ $\mathrm{ft}^{3}\left(73.37 \mathrm{~kg} / \mathrm{m}^{3}\right)$.

Tests were conducted on the Martin guarded hot plate facility in order to evaluate the heat transfer characteristics of the integral insulation/support concept. The specimen consisted of five 0.25 in. $(6.35 \mathrm{~mm})$ thick layers of flexible core and four layers of 0.005 in. $(0.127 \mathrm{~mm})$ aluminum foil. An additional foil layer was added to the top and bottom foils of the specimen. The total specimen weight was $0.305 \mathrm{lb}(0.138 \mathrm{~kg})$ for the 9 in. $(0.229 \mathrm{~m})$ diameter disc. This converts to a specimen density of $6.65 \mathrm{lb} / \mathrm{ft}^{3}\left(106.53 \mathrm{~kg} / \mathrm{m}^{3}\right)$. As in the microquartz tests, runs were made at two basic temperature levels such that the average temperatures in the specimens were $190^{\circ} \mathrm{F}$ and $440^{\circ} \mathrm{F}\left(361^{\circ} \mathrm{K}\right.$ and $\left.500^{\circ} \mathrm{K}\right)$. Tests were also conducted at various pressure levels to determine the sensitivity of the system to pressure. The test results are shown in Figure 62. For purposes of comparison, the test values for the microquartz insulation are also shown. It is seen that for the 1-atm case, the effective thermal conductivity of the honeycomb/foil concept is between 20 to $40 \%$ higher than the microquartz values. A 7\% difference in the relative densities of the systems tested exists and is in favor of the microquartz. A single test point was run on a 1.25 in. $(31.75 \mathrm{~mm}$ ) thick flexible core specimen with no radiation barriers and is also shown in Figure 62. It indicates that a significant reduction in the effective conductivity is realized through the use of the foil layers. Figure 63 defines the pressure sensitivity of the honeycomb/foil concept effective conductivity for the two average temperatures. Note that this concept is much less sensitive to atmospheric pressure changes than is the microquartz. 
In order to evaluate the design implications of the se thermal conductivity test data, a parametric study was performed to establish the interaction between insulation thickness, thermal conductivity, and peak structure temperature at touchdown. Since the overshoot re-entry trajectory dictates the required insulation thicknesses, it was chosen as a basis for the analysis. The maximum $\dot{\mathrm{q}}_{\mathrm{L}} / \dot{\mathrm{q}}_{\text {stag. }}$ ratio associated with the overshoot trajectory for vehicle areas wherein the panel concepts would be applied is 0.70 (ref. 3). For this study, the ablator chosen was LDNP since previous analysis indicates that for a given design point the bondline temperature peaks at $800^{\circ} \mathrm{F}\left(700^{\circ} \mathrm{K}\right)$, but earlier in time for the LDNP system than for the NASA 602 system, thereby providing a longer time period to touchdown for the structure to receive heat. The nominal thicknesses chosen for the analysis are taken from Figure 51 and are:

$$
\begin{aligned}
& \text { LDNP thickness }=1.20 \mathrm{in} .(30.48 \mathrm{~mm}) \\
& \text { Insulation thickncss }=1.34 \mathrm{in} .(34.04 \mathrm{~mm}) .
\end{aligned}
$$

A series of computer runs was conducted on the T-CAP III program by applying various correction factors to the nominal insulation thickness (1.34 in. or $34.04 \mathrm{~mm}$ ) and the nominal insulation thermal conductivity upon which the heat shield design curves were based. The results are shown in Figure 64. The carpet plot format of Figure 64a is useful for interpolation. Interpretation of Figure 64 with regard to the indicated increase in the microquartz thermal conductivity above the design values was performed as follows:

(1) From Figure 58 at $440^{\circ} \mathrm{F}\left(500^{\circ} \mathrm{K}\right)$ and $0.0105 \mathrm{~atm}$, test data indicates a $\mathrm{k} / \mathrm{k}_{\mathrm{nom}}=1.70$.

(2) From Figure 64, for $\mathrm{k} / \mathrm{k}_{\text {nom }}=1.70$, insulation thickness factor $=1.41$.

(3) Estimated average insulation thickness for the nominal conductivity $\left(\mathrm{k} / \mathrm{k}_{\mathrm{nom}}=1.0\right)$ is $1.4 \mathrm{in} .(35.56 \mathrm{~mm})$, based on Figure 52.

(4) Therefore, average thickness required for the test data conductivity $=1.98 \mathrm{in} .(50.29 \mathrm{~mm})$.

(5) The increased insulation thickness adds $0.30 \mathrm{lb} / \mathrm{ft}^{2}(1.464$ $\mathrm{kg} / \mathrm{m}^{2}$ ) to the insulation weight.

(6) For a $26.67 \mathrm{ft}(8.128 \mathrm{~m}) \mathrm{HL}-10$ vehicle, the total wetted surface area is approximately $829 \mathrm{ft}^{2}\left(77.01 \mathrm{~m}^{2}\right)$. Accord- 
ingly, the increase in heat shield weight due to the change in conductivity can be estimated as $248 \mathrm{lb}(112.5 \mathrm{~kg})$.

A similar application of Figure 64 to the te st data for the integral insulation/support concept indicates the use of this concept would impose a heat shield weight penalty of $0.76 \mathrm{lb} / \mathrm{ft}^{2}\left(3.71 \mathrm{~kg} / \mathrm{m}^{2}\right)$ or $630 \mathrm{lb}$ $(285.77 \mathrm{~kg})$ on the vehicle. In addition, the use of the honeycomb foil concept would increase the required insulation thickness by $67 \%$.

For purposes of this study, the microquartz thermal conductivity values, upon which the design curves are based, were retained. It is apparent however that the design of an insulation system for an operational heat shield configuration must be based on adequately validated data relating its temperature and atmospheric pressure sensitivity. Furthermore, the indicated mechanical pressure sensitivity of the quartz fiber insulation characteristics implies that a gap should be allowed between the substrate panel and insulation at installation, in order to preclude compression of the insulation by the panels under airload.

The integral insulation/support concept in its present form imposes a severe weight penalty on the system. Sufficient information is not available, nor was it within the scope of this program, to evaluate associated tradeoffs such as savings in vehicle structural weight, reduced number of attachments, and potential weight reduction in the substrate panel. Further development work on the concept is indicated in order to more thoroughly define its potential and, if feasible, generate a practical piece of hardware.

Analysis of panel support heat short problem. - One of the problems associated with the thermal design of a multipost supported thermostructural heat shield panel is the extent to which the insulating characteristics of the system are compromised by the presence of a series of panel support members penetrating the basic insulation system. Significant parameters influencing the heat short problem are support configuration and material, support spacing, and mass distribution in the basic vehicle structure. With regard to this last parameter, consideration of practical structural design techniques indicates that at each panel support/vehicle structure interface, some type of backup structure must be provided in order to redistribute the induced concentrated load. The requirement becomes more severe as the support spacing increases and, hence, the load per support increases. The backup structure may be in the form of frames, stringers, intercostals, etc., some of which may be required primarily to carry overall loads and happen to be properly oriented for purposes of panel support. A realistic appraisal of the heat short problem must account for this parameter since it can significantly reduce the heat short effect.

The analytical model for heat short thermal analysis is defined below and in Figure 65. 
(1) Support spacing $=7$ in. $(0.178 \mathrm{~m})$ constant.

(2) The mass of the aluminum backup structure "hard point" is variable. Equivalent cross sectional area of the backup member is based on its mass and the support spacing.

(3) The panel support is assumed to be a cup-type with dimensions shown in Figure 65. Both phenolic/glass laminate and stainless steel supports were analyzed.

(4) The overshoot re-entry trajectory and the same materials and thicknesses as used in the insulation optimization parametric study were used here. A two-dimensional heat flow analysis was conducted on the Martin FB-127 multidimensional heat flow program. The appropriate bond-line temperature time history as determined from T-CAP III analysis was used as the thermal forcing function.

Results of the heat short study are presented in Figure 66. Note that the phenolic/glass support creates a negligible heat short problem. 'This is due to the fact that although the support transfers more heat than the insulation, the rate at which the heat is introduced into the structure is sufficiently low to permit the high conductivity aluminum structure to diffuse it throughout its entire mass. This is borne out by the fact that the analysis results indicate no thermal gradient in a radial direction from the support. It is seen that for the steel support a substantial heat short problem is created. The effect of considering the backup structure is quite significant in reducing the peak temperatures. For the most severe case of a steel support with no backup structure, the maximum temperature is seen to be $227^{\circ} \mathrm{F}\left(382^{\circ} \mathrm{K}\right)$. The design implications of the heat short problem can be evaluated by going back to Figure 64 . From Figure 64 , it can be shown that an additional $0.67 \mathrm{in} .(17.02 \mathrm{~mm})$ of microquartz insulation is needed in order to reduce the peak temperature to $150^{\circ} \mathrm{F}\left(339^{\circ} \mathrm{K}\right)$. This is equivalent to a weight penalty of $0.347 \mathrm{lb} / \mathrm{ft}^{2}\left(1.693 \mathrm{~kg} / \mathrm{m}^{2}\right)$ or $287 \mathrm{lb}(130.2 \mathrm{~kg})$ per vehicle. Obviously, this penalty can be minimized by considering the mass of the backup structure.

Additional analyses were conducted based on varying the support spacing. Results are presented in Figure 67 for the case of no backup structure heat sink effects. The temperatures are shown at the panel support/structure interface.

\section{Substrate Panel Optimization and Structural Analysis}

Structural optimization studies. - Detailed structural optimization studies were performed on the substrate/ablator combinations selected 
in the initial screening study. The 1620 computer program used in the preliminary structural optimization study was modified where neces sary to conform to the following constraints:

(1) Maximum permissible ablator strains must not be exceeded (Figures 17 and 21).

(2) Allowable substrate material strength and stability limits must not be exceeded.

(3) Since aerodynamic smoothness requirements for lifting entry vehicles are not clearly defined, an arbitrary deflection criterion was applied. The maximum permissible deflection between supports was set at $0.02 \times$ support spacing. (Results of the study reveal that this criterion did not influence the panel requirements.)

(4) Minimum permissible gages for honeycomb panel faces were set at $0.020 \mathrm{in} .(0.508 \mathrm{~mm})$ for the plastic laminates and 0.005 in. $(0.127 \mathrm{~mm})$ for stainless steel. The stainless steel minimum gage constraint was increased above the 0.0015 in. $(0.0381 \mathrm{~mm})$ value used in the preliminary structural optimization in order to account for stretch forming limitations. For the relatively sharp radii associated with certain portions of the vehicle, the minimum gage constraint for stainless steel is optimistic.

(5) The minimum permissible depth of the honeycomb core was set at 0.1875 in. $(4.76 \mathrm{~mm})$ based on manufacturing capability.

Optimum substrate dimensions and weights were determined for substrate panel temperatures of $70^{\circ}, 300^{\circ}$, and $800^{\circ} \mathrm{F}\left(294^{\circ}, 422^{\circ}\right.$ and $\left.700^{\circ} \mathrm{K}\right)$. Honeycomb panel configurations with phenolic/glass, (Narmco 506 resin), phenyl-silane/glass (CTL 37-9x resin) and 177PH (TH1050) stainless steel were considered. Further, each substrate configuration was optimized for the room temperature case for LDNP and NASA 602 ablator thicknesses of $0.5,1.0$ and $1.5 \mathrm{in}$. (12.7, 25.4 and $38.1 \mathrm{~mm}$ ).

Typical results of the structural optimization study for the 0.5- and 1.5-in. (12.7 and $38.1 \mathrm{~mm}$ ) ablator thicknesses are presented in Figures 68 through 95. The optimization curves appear in two formats. One set defines the interrelationship between required panel face thickness, panel depth, and the critical design moment in the panel. Presenting the data in this form enables the critical constraint on panel optimization to be identified. For example, in Figure 68, the design boundaries imposed by the manufacturing restraint (minimum panel depth) and minimum face gage define the design "corridor" within which the panel may be optimized. Within these limits, it is seen that, in this case, the constraint on optimum panel design is the permissible ablator strain. The second 
format defines the required panel weight in terms of panel depth and critical design moment. Note that the substrate weights shown in the curves are exclusive of the adhesive bond weight. A unit weight of 0.42 $\mathrm{lb} / \mathrm{ft}^{2}\left(2.05 \mathrm{~kg} / \mathrm{m}^{2}\right)$ should be added to the plotted weights in order to account for three layers of $\mathrm{HT}^{-424}$ adhe sive. Here again, the manufacturing and minimum gage constraints define the design corridor, and it is seen that the optimum panel weight is attained at the intersection of the two corridor boundaries. For optimum design then, the critical moment in the panel is that which intersects the juncture of the corridor boundaries. For a given panel pressure loading, the critical moment in the panel is dependent upon the panel support spacing. Hence, the optimum panel design dictates the design moment which in turn dictates the support spacing. In order to establish a true optimum, weight tradeoffs between the panel and support weights should be considered. Information presented in the following subsection, Panel Support Spacing Requirements, relative to support weight factors indicates that no significant savings in weight can be gained by trading off between panel and support weights since practical support configurations, even in dense patterns, contribute only approximately $0.051 \mathrm{~b} / \mathrm{ft}^{2}\left(0.244 \mathrm{~kg} / \mathrm{m}^{2}\right)$ to the system weight. There may be specific design cascs, however, where a weight trade off could be significant. Operational considerations could to some extent influence the selection of an optimum panel design in the sense that although a given configuration may provide a minimum structural weight, the number of attachments required to realize that weight (at optimum panel design moment) could be much greater than the number required for a configuration which weighs a bit more. Therefore, from a standpoint of turnaround time and general refurbishability, or access to interior compartments, the slightly heavier design might be more desirable. An additional factor to be considered in establishing the support spacing is the location of existing hard points on the vehicle structure. Obviously, the vehicle structural design and heat shield support requirements must be closely coordinated in order to arrive at a satisfactory compromise.

Figures 96 through 99 illustrate the influence of changing substrate and ablator materials on the critical design constraints and optimum design moments. Note that changing the substrate material from stainless steel to phenolic/glass laminate eliminates intercell buckling as the critical constraint, and ablator strength becomes critical for design at the optimum weight. For the phenolic/glass laminate substrate, a change from NASA 602 to LDNP ablator makes ablator strength critical over a wider range of panel depth and weight. Figure 99 superimposes the design corridors for the three candidate substrate materials. Inspection reveals that, although the optimum weights are fairly comparable, the phenolic/glass substrate is lighter. 
Another yardstick by which to evaluate the relative capabilities of the substrate panel materials is the critical moment at the optimum design point. As noted previously, as the optimum design moment increases, the required number of supports decreases. Table 24 and Figure 100 present comparisons of optimum design moments for phenolic/ glass and stainless steel. For the room temperature case, the phenolic/ glass and phenyl-silane/glass optimum moments are nearly identical.

\section{TABLE 24. REPRESENTATIVE OPTIMUM MOMENTS FOR PANEL DESIGN AT $70^{\circ} \mathrm{F}\left(294^{\circ} \mathrm{K}\right)$}

\begin{tabular}{|c|c|c|c|c|c|c|}
\hline \multirow{5}{*}{$\begin{array}{c}\text { Substrate } \\
\text { panel } \\
\text { material }\end{array}$} & \multirow{4}{*}{\multicolumn{2}{|c|}{$\begin{array}{l}\text { Ablator } \\
\text { thickness }\end{array}$}} & \multicolumn{4}{|c|}{ Optimum moments } \\
\hline & & & \multicolumn{4}{|c|}{ Ablator material } \\
\hline & & & \multicolumn{2}{|c|}{ LDNP } & \multicolumn{2}{|c|}{ NASA 602} \\
\hline & & & \multirow{2}{*}{$\frac{\text { in. }-1 b}{\text { in. }}$} & \multirow{2}{*}{$\frac{\mathrm{cm}-\mathrm{N}}{\mathrm{cm}}$} & \multirow{2}{*}{$\frac{\text { in. }-1 b}{\text { in. }}$} & \multirow{2}{*}{$\frac{\mathrm{cm}-\mathrm{N}}{\mathrm{cm}}$} \\
\hline & in. & $\mathrm{mm}$ & & & & \\
\hline $\begin{array}{l}\text { 17-7PH } \\
\text { (TH1050) }\end{array}$ & $\begin{array}{l}0.50 \\
1.50\end{array}$ & $\begin{array}{l}12.7 \\
19.05\end{array}$ & $\begin{array}{l}46 \\
27\end{array}$ & $\begin{array}{l}205 \\
120.1\end{array}$ & $\begin{array}{l}46 \\
46\end{array}$ & $\begin{array}{l}204.6 \\
204.6\end{array}$ \\
\hline $\begin{array}{l}\text { Phenolic/ } \\
\text { glass laminate }\end{array}$ & $\begin{array}{l}0.50 \\
1.50\end{array}$ & $\begin{array}{l}12.7 \\
19.05\end{array}$ & $\begin{array}{l}60 \\
22\end{array}$ & $\begin{array}{r}266.9 \\
97.9\end{array}$ & $\begin{array}{r}210 \\
84\end{array}$ & $\begin{array}{l}934.1 \\
373.6\end{array}$ \\
\hline
\end{tabular}

Note that the highest optimum design moments are for the phenolic/ glass honeycomb substrate with the NASA 602 ablator. Generally, the optimum design moments are higher for the phenolic/glass substrates as opposed to steel. The exception occurs when comparing the substrates with the LDNP thicknesses of $0.84 \mathrm{in} .(2.13 \mathrm{~cm})$ or greater. The phenolic/glass substrate has a slightly lower optimum design moment due to the low allowable strain of the LDNP. To interpret the se optimum design moments in terms of panel support requirements, the curves of Figure 102 were employed (see following paragraphs, Panel Support Spacing Requirements). For a support spacing aspect ratio of 1.0 and a panel design overpressure of $12 \mathrm{psi}\left(82.74 \mathrm{kN} / \mathrm{m}^{2}\right)$, the $\mathrm{re}^{-}$ quired support spacings were determined for the optimum design moments as defined in Table 24 and Figure 100. The support requirements are defined in Table 25 and Figure 101. 
TABLE 25. COMPARISON OF TYPICAL PANEL SUPPORT REQUIREMENTS AT $70^{\circ} \mathrm{F}\left(294^{\circ} \mathrm{K}\right)$

\begin{tabular}{|c|c|c|c|c|c|c|}
\hline \multirow{4}{*}{$\begin{array}{c}\text { Substrate } \\
\text { panel } \\
\text { material }\end{array}$} & & & \multicolumn{4}{|c|}{ Support requirements } \\
\hline & \multirow{2}{*}{\multicolumn{2}{|c|}{$\begin{array}{l}\text { Ablator } \\
\text { thickness }\end{array}$}} & \multicolumn{4}{|c|}{ Ablator material } \\
\hline & & & \multicolumn{2}{|c|}{ LDNP } & \multicolumn{2}{|c|}{ NASA 602} \\
\hline & in. & $\mathrm{mm}$ & per $\mathrm{ft}^{2}$ & per $\mathrm{m}^{2}$ & per $\mathrm{ft}^{2}$ & per $\mathrm{m}^{2}$ \\
\hline $\begin{array}{l}17-7 \mathrm{PH} \\
(\mathrm{TH} 1050)\end{array}$ & $\begin{array}{l}0.50 \\
1.50\end{array}$ & $\begin{array}{l}12.7 \\
19.05\end{array}$ & $\begin{array}{l}7.72 \\
13.7\end{array}$ & $\begin{array}{r}83.10 \\
147.41\end{array}$ & $\begin{array}{l}7.96 \\
7.96\end{array}$ & $\begin{array}{l}85.65 \\
85.65\end{array}$ \\
\hline $\begin{array}{l}\text { Phenolic/glass } \\
\text { laminate }\end{array}$ & $\begin{array}{l}0.50 \\
1.50\end{array}$ & $\begin{array}{l}12.7 \\
19.05\end{array}$ & $\begin{array}{l}6.15 \\
16.2\end{array}$ & $\begin{array}{r}66.17 \\
174.0\end{array}$ & $\begin{array}{l}1.77 \\
4.37\end{array}$ & $\begin{array}{l}19.05 \\
47.02\end{array}$ \\
\hline
\end{tabular}

It is significant to note that for the case of the NASA 602 ablator, the number of supports required for the phenolic/glass substrate is significantly fewer than the number of supports required by the steel substrate, depending upon ablator thickness.

Panel support spacing requirements. - The relationship between support spacing, panel design overpressure and panel design moment was generated by the method of Timoshenko (ref. 6). For this particular case it was assumed that the support reacted the panel load over a circular area, the radius of which is $0.05 \mathrm{x}$ support spacing. Curves defining the relationship are presented in Figures 102 and 103 for two support spacing aspect ratios.

In order to gain a quantitative evaluation of the significance of the panel supports in terms of weight, a detail stress analysis was conducted on two post-type support designs, consisting of a flanged cup configuration and a hat section member. The results are prescnted in Figures 104 and 105. The design case was a $19.45 \mathrm{ft}^{2}\left(1.81 \mathrm{~m}^{2}\right)$ panel at room temperature exposed to design overpressures of 2 and 6 psi $\left(13.79\right.$ and $\left.41.37 \mathrm{kN} / \mathrm{m}^{2}\right)$. The results are shown in terms of total attachment weight versus attachment load and indicate that, except at very low attachment loads (large number of attachments), the attachments comprise a small percentage of the total system weight.

Attachment of the substrate panel to the basic vehicle shell by means of any practical point support system would necessitate penetration and, subsequently, a structural discontinuity in the panel. In the case of a honeycomb panel, the panel support itself could be designed to provide local reinforcement to the panel inner face by bonding the cup flange directly to the penetrated area. The outer face, which would be more critical under a design overpressure, would 
require the addition of a local doubler or, possibly, the ablator itself might provide sufficient reinforcement for the critical design pressures, which are normally associated with launch abort situations wherein most of the ablator would be at or near room temperature. Local discontinuity problems may or may not be significant depending upon the materials, geometry and types of loading involved. Standard analytical methods are available for evaluating stress concentration problems and should be supported by element tests.

Panel flutter considerations. - Flutter design criteria for multipost supported substrate panel/ablator composites were established based upon available experimental data.

Experimental results for the flutter of flat rectangular plates are defined in ref. 8 and are represented in Figure 106 for use as a flutter boundary criteria.

In considering post supported panels, the panel boundaries were taken to be that portion of the panel bounded by a set of four supports of streamwise spacing (a) being the equivalent length and spanwise spacing (b) the equivalent panel width. This panel breakdown was based on the assumption that the most critical mode of flutter will be the simply supported modes bordered by the posts (line of spanwise posts being a node line). As indicated in ref. 9 for many streamwise bays and infinite width the number of bays is not important, with the single bay solution being the most critical flutter solution when damping is included.

Based on these assumptions, a curve of the minimum equivalent panel thickness required to preclude flutter is given in Figure 107. These curves were determined from an envelope of maximum dynamic pressure at various Mach numbers for the HL-10 trajectories in reference 3. The trajectories considered in establishing the flutter design envelope were: Nominal Ascent, Maximum Heat Rate Abort, Maximum Dynamic Pressure Abort, Maximum Load Factor Abort, and Nominal, Overshoot, and Undershoot Re-entry. It is seen that a maximum value of flutter parameter occurs at Mach 1.5 and is due to the Maximum Dynamic Pressure Abort Trajectory. Panel designs should be based on this maximum flutter parameter in order to assure compatibility with all of the operational mission requirements.

A method for including the effects of panel curvature in the flutter design criteria is to utilize the previously defined boundary criteria with an adjusted equivalent panel thickness. This thickness would correspond to a flat panel thickness required to give the same critical buckling stress as that of the curved panel. Based on data in ref. 10 the curvature correction factored was established and is presented in Figure 108. 
Another factor influencing the flutter characteristics of panels is pressure differentials across the panel. Independent experimental investigations have shown that small amounts of differential pressure can significantly increase the flutter dynamic pressure. For example, test results of Titan I test panels (ref. 11) show that a differential pressure of $0.2 \mathrm{psi}\left(137.9 \mathrm{daN} / \mathrm{m}^{2}\right)$ is sufficient to preclude flutter for the design trajectory. Similar results are shown in ref. 12.

The inclusion of stiffness and structural damping in the panels afforded by the ablator material and the probability of differential pressure across the panels tend to mitigate the flutter problem. Based on these factors, the defined flutter boundary criteria should be conservative.

\section{Application of Design Data}

The design data defined in the previous subsections were applied to selected panel areas of the HL-10 vehicle in order to evaluate the composite thermostructural panel design requirements for a typical manned lifting entry vehicle as represented by the NASA Langley HL-10 configuration.

Panel identification and environmental definition.- Based on the heat shield panel orientation define $\bar{d}$ in the subsection on vehicle size and geometry under Analysis and Optimization of Selected Thermostructural Panel Concepts, three panels were selected as being representative of the design extremes that would be encountered on the HL-10 configuration and are defined in Figure 109. The leading edge panel was selected since it is exposed to the most severe load and thermal environment of any panel on the vehicle. In addition, this panel has one of the highest degrees of curvature. The crown panel (upper surface) is the largest panel on the vehicle and is exposed to the low order thermal and load environments that are typical of the vehicle upper surface. The bottom panel is representative of the average design requirements for panels of this type. Most of the analysis and design application studies were performed on the leading edge and crown panels since they represent the extreme design cases.

Based on the heating rate distributions defined in ref. 3, a heating $r$ ate ratio envelope can be defined for any panel. Figures 110 and 111 present the applicable envelopes for each of the sample panels for the nominal and overshoot re-entry cases. These data can be used to define the heat shield thickness range associated with each panel.

Based on ref. 3 pressure distribution data and supplementary NASA Langley wind tunnel test data, the flight environment spectrum 
of the HL-10 vehicle was surveyed and a set of critical design pressures for the sample panels were defined. The pressures were based on the assumption that the insulation space between the substrate panel and the vehicle structure is vented to a point on the vehicle upper surface toward the aft end. Further, it was assumed that the pressure coefficient at the vent location was -0.40 and that no venting lag existed. The resulting design pressures are defined in Table 26.

TABLE 26. CRITICAL LIMIT DESIGN PRESSURES AND TEMPERATURES FOR SUBSTRATE PANEL DESIGN

\begin{tabular}{|c|c|c|c|c|c|}
\hline \multirow[b]{2}{*}{ Panel } & \multicolumn{2}{|c|}{$\begin{array}{c}\text { Limit } \\
\text { pressure }\end{array}$} & \multicolumn{2}{|c|}{$\begin{array}{c}\text { Maximum } \\
\text { substrate } \\
\text { temperature }\end{array}$} & \multirow[b]{2}{*}{ Condition } \\
\hline & psi & $\mathrm{kN} / \mathrm{m}^{2}$ & ${ }^{\circ} \mathrm{F}$ & ${ }^{\circ} \mathrm{K}$ & \\
\hline $\begin{array}{l}\text { Leading } \\
\text { edge }\end{array}$ & $\begin{array}{l}8.5 \\
6.64 \\
2.60 \\
\end{array}$ & $\begin{array}{l}58.61 \\
\\
45.78 \\
17.93 \\
\end{array}$ & $\begin{array}{l}125 \\
280 \\
800 \\
\end{array}$ & $\begin{array}{l}325 \\
411 \\
700 \\
\end{array}$ & $\begin{array}{l}\text { Maximum load factor } \\
\text { abort } \\
\text { Undershoot re-entry } \\
\text { Nominal re-entry } \\
\end{array}$ \\
\hline Bottom & $\begin{array}{l}5.85 \\
4.64 \\
1.82\end{array}$ & $\begin{array}{l}40.33 \\
31.99 \\
12.54\end{array}$ & $\begin{array}{l}125 \\
280 \\
800\end{array}$ & $\begin{array}{l}325 \\
411 \\
700\end{array}$ & $\begin{array}{l}\text { Maximum load factor } \\
\text { abort } \\
\text { Undershoot re-entry } \\
\text { Nominal re-entry }\end{array}$ \\
\hline Crown & $\begin{array}{r}1.15 \\
0.44 \\
0.23 \\
-0.44\end{array}$ & $\begin{array}{r}7.93 \\
3.03 \\
1.59 \\
-3.03\end{array}$ & $\begin{array}{l}280 \\
800 \\
620\end{array}$ & $\begin{array}{l}325 \\
411 \\
700 \\
600\end{array}$ & $\begin{array}{l}\text { Maximum dynamic pres- } \\
\text { sure abort } \\
\text { Undershoot re-entry } \\
\text { Nominal re-entry } \\
\text { Overshoot re-entry }\end{array}$ \\
\hline
\end{tabular}

For purposes of structural analysis, the limit pressures shown in Table 25 were applied in combination with a safety factor of 1.50.

Panel design comparisons based on optimization studies.- For the three sample panels, the results of the structural optimization study were applied in order to evaluate the interrelationship between honeycomb substrate panel depth and support spacing. It was assumed that the substrate panel material was phenolic/glass laminate. The maximum and minimum ablator thicknesses on each panel were determined from the heating rate ratio envelopes and the heat shield design curves and are defined in Table 27. 
TABLE 27. ABLATOR THICKNESS RANGES FOR SAMPLE PANELS

\begin{tabular}{|c|c|c|c|c|c|c|c|c|c|c|}
\hline \multirow[b]{3}{*}{ Item } & \multicolumn{4}{|c|}{ Leading edge panel } & \multicolumn{4}{|c|}{ Bottom panel } & \multirow{2}{*}{\multicolumn{2}{|c|}{$\begin{array}{l}\text { Crown } \\
\text { panel } \\
\text { All points }\end{array}$}} \\
\hline & \multicolumn{2}{|c|}{ Sta 19.2} & \multicolumn{2}{|c|}{ Sta 80} & \multicolumn{2}{|c|}{ Sta 80} & \multicolumn{2}{|c|}{ Sta 152} & & \\
\hline & in. & $\mathrm{cm}$ & in. & $\mathrm{cm}$ & in. & $\mathrm{cm}$ & in. & $\mathrm{cm}$ & in. & $\mathrm{cm}$ \\
\hline (NASA 602) & 1.39 & 3.53 & 1.14 & 2.90 & 1.07 & 2.72 & 0.95 & 2.41 & 0.36 & 0.91 \\
\hline${ }^{t} \max$ & & & & & & & & & & \\
\hline (NASA 602) & 0.96 & 2.44 & 0.89 & 2.26 & 0.96 & 2.44 & 0.74 & 1.88 & 0.36 & 0.91 \\
\hline$\left({ }_{\text {LDNP }}^{t_{\min }} \mathrm{t}_{\max }\right.$ & 1. 47 & 3.73 & 1.06 & 2.69 & 0.98 & 2.49 & 0.81 & 2. 06 & 0.34 & 0.86 \\
\hline$(\mathrm{LDNP}) \mathrm{t}_{\min }$ & 0.86 & 2.18 & 0.79 & 2.00 & 0.82 & 2.08 & 0.62 & 1.57 & 0.34 & 0.86 \\
\hline
\end{tabular}

The ablator thicknesses in Table 27 were used as a basis for selecting which substrate optimization curve to use for the design cases wherein ablator strain was a factor. Based on a support spacing aspect ratio of 1.5 , the required support spacings for panel depths of 0.2275 in. $(5.78 \mathrm{~mm}), 0.25$ in. $(6.35 \mathrm{~mm})$ and 0.375 in. $(9.52 \mathrm{~mm})$ were determined. All of the design cases presented in Table 26 were surveyed, and it was determined that the leading edge and bottom panel designs were critical for the maximum load factor abort case, and the crown panel was critical for the maximum dynamic pressure abort case. Typical results are shown in Table 28 for the point of maximum ablator thickness in each phenolic/glass substrate panel. Note that the required support spacings are for the critical panel design case and the value shown is the short dimension (assumed aspect ratio $=1.50$ ).

TABLE 28. SUPPORT SPACING REQUIREMENTS FOR VARIOUS PANEL DEPTHS AND ABLATORS

\begin{tabular}{|c|c|c|c|c|c|c|c|c|c|c|c|c|c|}
\hline \multirow{2}{*}{\multicolumn{2}{|c|}{$\begin{array}{c}\text { Substrate } \\
\text { panel depth }\end{array}$}} & \multicolumn{4}{|c|}{ Leading edge panel } & \multicolumn{4}{|c|}{ Bottom panel } & \multicolumn{4}{|c|}{ Crown panel } \\
\hline & & \multicolumn{2}{|c|}{ LDNP } & \multicolumn{2}{|c|}{ NASA 602} & \multicolumn{2}{|c|}{ LDNP } & \multicolumn{2}{|c|}{ NASA 602} & \multicolumn{2}{|c|}{ LDNP } & \multicolumn{2}{|c|}{ NASA 602} \\
\hline in. & $\mathrm{mm}$ & in. & $\mathrm{cm}$ & in. & $\mathrm{cm}$ & in. & crn & in. & $\mathbf{c m}$ & in. & $\mathrm{cm}$ & in. & $\mathrm{cm}$ \\
\hline 0.2275 & 5.78 & 2.08 & 5. 28 & 4. 16 & 10.57 & 3. 13 & 7.95 & 5.96 & 15.14 & 9.44 & 23.98 & 18. 50 & 46.99 \\
\hline 0.25 & 6.35 & 2.30 & 5.84 & 4.55 & 11.56 & 3. 30 & 8. 38 & 6.68 & 16.97 & 10.14 & 25.76 & 19.90 & 50.55 \\
\hline 0.375 & 9.52 & 3.31 & 8. 41 & 6.66 & 16.92 & 4. 83 & 12.27 & 9.59 & 24.36 & 14.23 & 36. 14 & 28. 50 & 72.39 \\
\hline
\end{tabular}


As would be expected, the required support spacing increases with increasing panel depth. By increasing the panel depth from 0.25 in. $(6.35 \mathrm{~mm})$ to $0.375 \mathrm{in} .(9.52 \mathrm{~mm})$, the support spacing can be increased by approximately $45 \%$. Table 28 also shows that for any given panel depth, the support spacing required for NASA 602 system is approximately twice as great as the requirement for the LDNP system. The significance of this relationship is more meaningful when expressed in terms of the required number of attachments per unit area as shown in Table 29.

\section{TABLE 29. COMPARATIVE SUPPORT DISTRIBUTION DENSITIES}

\begin{tabular}{|c|c|c|c|c|c|c|c|c|c|c|c|c|c|}
\hline \multirow{3}{*}{\multicolumn{2}{|c|}{$\begin{array}{c}\text { Substrate } \\
\text { panel depth }\end{array}$}} & \multicolumn{4}{|c|}{ Leading edge panel } & \multicolumn{4}{|c|}{ Bottom panel } & \multicolumn{4}{|c|}{ Crown panel } \\
\hline & & \multicolumn{2}{|c|}{ LDNP } & \multicolumn{2}{|c|}{ NASA 602} & \multicolumn{2}{|c|}{ LDNP } & \multicolumn{2}{|c|}{ NASA 602} & \multicolumn{2}{|c|}{ LDNP } & \multicolumn{2}{|c|}{ NASA 602} \\
\hline & & $\begin{array}{l}\text { per } \\
\mathrm{ft}^{2}\end{array}$ & $\begin{array}{l}\text { per } \\
\mathrm{m}^{2}\end{array}$ & $\begin{array}{l}\text { per } \\
\mathrm{ft}^{2}\end{array}$ & $\begin{array}{l}\text { per } \\
\mathrm{m}^{2}\end{array}$ & $\begin{array}{l}\text { per } \\
\mathrm{ft}^{2}\end{array}$ & $\begin{array}{l}\text { per } \\
\mathrm{m}^{2}\end{array}$ & $\begin{array}{l}\text { per } \\
\mathrm{ft}^{2}\end{array}$ & $\begin{array}{l}\text { per } \\
\mathrm{m}^{2}\end{array}$ & $\begin{array}{l}\text { per } \\
\mathrm{ft}^{2}\end{array}$ & $\begin{array}{l}\text { per } \\
\mathrm{m}^{2}\end{array}$ & $\begin{array}{c}\text { per } \\
\mathrm{ft}^{2}\end{array}$ & $\begin{array}{l}\text { per } \\
\mathrm{m}^{2}\end{array}$ \\
\hline 0.2275 & 5.78 & 22.2 & 238.9 & 5.55 & 59.7 & 9.8 & 105.4 & 2. 70 & 29.1 & 1.08 & 11.6 & 0.28 & 3.0 \\
\hline 0.25 & 6.35 & 18.1 & 194.8 & 4.63 & 49.8 & 8.75 & 94.2 & 2.15 & 23.1 & 0.93 & 10.0 & 0.24 & 2.6 \\
\hline 0.375 & 0.62 & 8. 75 & 04.2 & 2.16 & 23. 2 & 4. 11 & 44.2 & 1.03 & 11.1 & 0.47 & 5.1 & 0.12 & 1.3 \\
\hline
\end{tabular}

It is seen that by increasing the panel depth from 0.25 in. $(6.35 \mathrm{~mm})$ to $0.375 \mathrm{in} .(9.52 \mathrm{~mm})$, the support requirements are cut in half. Furthermore, for a given panel depth, changing the ablator material from NASA 602 to LDNP increased the required number of supports by a ratio of $4 / 1$. A tradeoff study was performed to evaluate the substrate weight penalty imposed on the LDNP system by reducing the number of supports to equal that of the NASA 602 system. Two panel locations were examined for their respective critical design cases; the results are presented in Table 30 . It was assumed that for the case of the 4/1 support requirement ratio, the substrate panel depth was 0.375 in. $(9.52 \mathrm{~mm})$ (phenolic/glass laminate). The required increase in panel depth and weight for the LDNP system with reduced attachment spacing was then determined.

\section{TABLE 30. ATTACHMENT RATIO AND SUBSTRATE} PANEL WEIGHT TRADEOFF

\begin{tabular}{|l|c|c|c|c|c|c|c|c|}
\hline \multirow{4}{*}{$\begin{array}{l}\text { Ablator } \\
\text { system }\end{array}$} & \multicolumn{3}{|c|}{$\begin{array}{c}\text { Leading edge panel--Sta 19.2 } \\
\text { Attachment ratio }\end{array}$} & \multicolumn{3}{c|}{$\begin{array}{c}\text { Crown panel--all points } \\
\text { Attachment ratio }\end{array}$} \\
\cline { 2 - 9 } & \multicolumn{2}{|c|}{$4 / 1$} & \multicolumn{2}{|c|}{$1 / 1$} & \multicolumn{2}{|c|}{$4 / 1$} & \multicolumn{2}{|c|}{$1 / 1$} \\
\hline LDNP & 0.94 & 4.59 & 1.13 & 5.51 & 0.94 & 4.59 & 1.18 & 5.76 \\
NASA 602 & 0.94 & 4.59 & 0.94 & 4.59 & 0.94 & 4.59 & 0.94 & 4.59 \\
\hline
\end{tabular}


It is seen that for an LDNP system with the same number of attachments as the NASA 602 system, the LDNP heat shield substrate panel weight is approximately $0.2 \mathrm{lb} / \mathrm{ft}^{2}\left(0.98 \mathrm{~kg} / \mathrm{m}^{2}\right)$ heavier than that for the NASA 602 system. Based on the total vehicle wetted area, the weight penalty is $165 \mathrm{lb}(74.8 \mathrm{~kg})$ per vehicle.

Based on Figure 104, the contribution of the panel supports to the overall system weight was evaluated for a 0.375 in. $(9.52 \mathrm{~mm})$ phenolic/ glass substrate panel with NASA 602 ablator. The appropriate support requirements in Table 29 were used for the leading edge and crown panels. Results show that for the leading edge panel the support unit weight is $0.08 \mathrm{lb} / \mathrm{ft}^{2}\left(0.39 \mathrm{~kg} / \mathrm{m}^{2}\right)$ and for the crown panel, the weight is $0.0065 \mathrm{lb} / \mathrm{ft}^{2}\left(0.032 \mathrm{~kg} / \mathrm{m}^{2}\right)$, indicating that, since a major portion of the vehicle surface area is in relatively low pressure loading regions, the support weight contribution to the total vehicle weight is very small.

A comparison of the phenolic/glass and phenyl-silane/glass laminate substrate panels was performed using the structural optimization curves and design pressures previously defined. It will be recalled (see Figure 7) that the phenyl-silane/glass system retained higher strength properties at elevated temperatures when compared with the phenolic/glass systems. However, a comparison of the two at room temperature shows the phenolic/glass system to be superior. Typical comparisons of the two materials are shown in Tables 31 and 32 ; they are for the case of a $0.375 \mathrm{in.}(9.52 \mathrm{~mm})$ leading edge and crown substrate panel and a NASA 602 ablator. The problem was approached in terms of the required support spacing as a function of substrate panel material and design condition.

TABLE 31. COMPARISON OF PHENOLIC/GLASS AND PHENYLSILANE/GLASS LEADING EDGE SUBSTRATE PANELS

\begin{tabular}{|c|c|c|c|c|c|c|c|}
\hline \multirow[b]{2}{*}{ Material } & \multicolumn{2}{|c|}{$\begin{array}{c}\text { Tem- } \\
\text { perature }\end{array}$} & \multicolumn{2}{|c|}{$\begin{array}{l}\text { Ultimate } \\
\text { design } \\
\text { pressure } \\
\end{array}$} & \multicolumn{2}{|c|}{$\begin{array}{c}\text { Support } \\
\text { spacing } \\
\text { requirements }\end{array}$} & \multirow[b]{2}{*}{ Condition } \\
\hline & ${ }^{\circ} \mathrm{F}$ & ${ }^{\circ} \mathrm{K}$ & psi & $\mathrm{kN} / \mathrm{m}^{2}$ & in. & $\mathrm{m}$ & \\
\hline $\begin{array}{l}\text { Phenolic/glass } \\
\text { Phenyl-silane/ } \\
\text { glass }\end{array}$ & $\begin{array}{l}125 \\
125\end{array}$ & $\begin{array}{l}325 \\
325\end{array}$ & $\begin{array}{l}12.75 \\
12.75\end{array}$ & $\begin{array}{l}87.91 \\
87.91\end{array}$ & $\begin{array}{l}6.66 \\
6.34\end{array}$ & $\begin{array}{l}0.169 \\
0.161\end{array}$ & $\begin{array}{l}\text { Max. load } \\
\text { factor abort }\end{array}$ \\
\hline $\begin{array}{l}\text { Phenolic/glass } \\
\text { Phenyl-silane/ } \\
\text { glass }\end{array}$ & $\begin{array}{l}800 \\
800\end{array}$ & $\begin{array}{l}700 \\
700\end{array}$ & $\begin{array}{l}3.9 \\
3.9\end{array}$ & $\begin{array}{l}26.89 \\
26.89\end{array}$ & $\begin{array}{l}9.47 \\
14.0\end{array}$ & $\begin{array}{l}0.241 \\
0.356\end{array}$ & $\begin{array}{l}\text { Nominal } \\
\text { re-entry }\end{array}$ \\
\hline
\end{tabular}


TABLE 32. COMPARISON OF PHENOLIC/GLASS AND PHENYL-SILANE/GLASS CROWN SUBSTRATE PANELS

\begin{tabular}{|c|c|c|c|c|c|c|c|}
\hline \multirow[b]{2}{*}{ Material } & \multicolumn{2}{|c|}{$\begin{array}{c}\text { Tem- } \\
\text { perature }\end{array}$} & \multicolumn{2}{|c|}{$\begin{array}{l}\text { Ultimate } \\
\text { design } \\
\text { pressure }\end{array}$} & \multicolumn{2}{|c|}{$\begin{array}{c}\text { Support } \\
\text { spacing } \\
\text { requirements }\end{array}$} & \multirow[b]{2}{*}{ Condition } \\
\hline & ${ }^{\circ} \mathrm{F}$ & ${ }^{\circ} \mathrm{K}$ & psi & $\mathrm{kN} / \mathrm{m}^{2}$ & in. & $\mathrm{m}$ & \\
\hline $\begin{array}{l}\text { Phenolic/glass } \\
\text { Phenyl-silane/ } \\
\text { glass }\end{array}$ & $\begin{array}{l}125 \\
125\end{array}$ & $\begin{array}{l}325 \\
325\end{array}$ & $\begin{array}{l}1.725 \\
1.725\end{array}$ & $\begin{array}{l}11.89 \\
11.89\end{array}$ & $\begin{array}{l}25.9 \\
21.3\end{array}$ & $\begin{array}{l}0.658 \\
0.541\end{array}$ & $\begin{array}{l}\text { Max. dynamic } \\
\text { pressure abort }\end{array}$ \\
\hline $\begin{array}{l}\text { Phenolic/glass } \\
\text { Phenyl-silane/ } \\
\text { glass }\end{array}$ & $\begin{array}{l}800 \\
800\end{array}$ & $\begin{array}{l}700 \\
700\end{array}$ & $\begin{array}{l}0.346 \\
0.346\end{array}$ & $\begin{array}{l}2.39 \\
2.39\end{array}$ & $\begin{array}{l}31.8 \\
47.1\end{array}$ & $\begin{array}{l}0.808 \\
1.20\end{array}$ & $\begin{array}{l}\text { Nominal } \\
\text { re-entry }\end{array}$ \\
\hline
\end{tabular}

The abort case is seen to dictate the required spacing for the cases considered. For the leading edge panel, the phenolic/glass system permits a slightly greater support spacing than the phenyl-silane system. In this case, the design is ablator strain critical; therefore, the significant difference in the material strengths at this temperature does not affect the design to a great extent. However, for the case of the crown panel, the low ablator thickness eliminates ablator strain as a design constraint and the material strength becomes critical. Table 32 shows a significant difference in attachment spacing with the advantage toward the phenolic/glass system. The phenyl-silane glass system requires $50 \%$ more attachments per unit area than the phenolic/glass substrate panel. The se studies were based on a support aspect ratio of 1.5 . If the loading environment were more severe at the higher temperatures, an advantage might be shown for the phenyl-silane/glass system. However, on the basis of the predicted environment, the phenolic/glass substrate is the more efficient configuration.

Network structural analysis. - In order to establish typical stress fields in a post supported substrate panel under lateral pressure and temperature gradients, an existing digital program which employs a matrix-force method for analysis of complex structural components was employed. The program is defined in ref. 7. The leading edge panel was chosen for analysis and two critical cases were considered:

(1) Maximum load factor abort, ultimate design pressure = 12. $75 \mathrm{psi}\left(87.91 \mathrm{kN} / \mathrm{m}^{2}\right)$

(2) Nominal re-entry trajectory at time $=1800 \mathrm{sec}$ (critical for substrate panel thermal stresses).

The leading edge panel was broken down into the network defined in Figure 112. Not shown for reasons of clarity but included in the analysis was an additional network representing the aluminum structure. 
The substrate panel/aluminum structure interactions were accounted for by assigning appropriate elastic constants to the panel supports, which were assumed to be rigid type as opposed to flexible. The substrate panel was considered to be a $0.375 \mathrm{in.}(9.52 \mathrm{~mm})$ deep phenolic/ glass laminate honeycomb configuration with 0.02 in. $(0.504 \mathrm{~mm})$ faces. Three rows of five supports each comprise the support pattern. Peripheral support spacing was $8.35 \mathrm{in.}(0.212 \mathrm{~m})$ and the longitudinal support spacing was $14 \mathrm{in}$. $(0.356 \mathrm{~m})$. The standoff distance between the panel and aluminum structure was $1.3 \mathrm{in.}(3.3 \mathrm{~cm})$. For the thermal stress case, the temperature difference between the outer and inner faces was $65^{\circ} \mathrm{F}\left(36^{\circ} \mathrm{K}\right)$, with the outer face at $715^{\circ} \mathrm{F}\left(651^{\circ} \mathrm{K}\right)$ at the forward end of the panel and $40^{\circ} \mathrm{F}\left(22.2^{\circ} \mathrm{K}\right)$ with the outer face at $790^{\circ} \mathrm{F}\left(694^{\circ} \mathrm{K}\right)$ at the aft end. Furthermore, the temperature gradient along the length of the panel spanned $75^{\circ} \mathrm{F}\left(41.7^{\circ} \mathrm{K}\right)$ on the outer face and $100^{\circ} \mathrm{F}\left(55.6^{\circ} \mathrm{K}\right)$ on the inner face. In both cases, the ablator was considered nonstructural.

Results of the analysis are presented in Figures 113 and 114. Analysis revealed that for the critical airload case, no thermal stresses exist in the substrate. For the critical thermal stress case, the concurrent load induced stresses are less than 10\% of the thermal stresses. As would be expected, the presence of the post type supports exerts a significant influence on the panel thermal stress distributions. The effect becomes more pronounced at support points further away from the center of the panel, indicating that for rigid supports, a panel size constraint also exists and is a function of the panel and support strength at temperature. It is also indicated that this constraint is not as severe as that for the case of flexible supports and steel panels, since the maximum permissible size for the steel panels has been shown to be approximately half as large as the phenolic/glass panel (page 37).

Compatibility with flutter envelope.-Based on the flutter envelope presented in Figure 107 , it was determined that flutter requirements impose no constraints upon the panel design. The critical flutter design case is the maximum dynamic pressure abort trajectory. For a 0.375 in. $(9.52 \mathrm{~mm})$ phenolic/glass substrate panel and a support spacing aspect ratio of 1.5 , a maximum permissible support spacing (streamwise) of $50.3 \mathrm{in.}(1.28 \mathrm{~m})$ is allowed in order to preclude panel flutter. For a $0.25 \mathrm{in.}(6.35 \mathrm{~mm})$ phenolic/glass substrate pane1, the critical spacing is $37.6 \mathrm{in.}(0.955 \mathrm{~mm})$. Since the spacing requirements for the panel pressure loading cases are more stringent and the effects of panel curvature and ablator dampening are not considered, it is concluded that panel flutter requirements are not critical for design.

Thermal stresses. - Representative calculations of thermal stresses in the ablator, substrate panel, and aluminum structure were made for the three conditions defined below:

(1) Room temperature thermal stresses induced by bonding and curing composite panels at elevated temperatures 
(2) Orbital cold soak

(3) Undershoot re-entry after orbital cold soak.

The maximum ablator thickness, as required on the leading edge panel, and the minimum thickness, as required on the crown panel, were analyzed for both ablators in conjunction with a 0.375 in. (9.52 $\mathrm{mm}$ ) depth phenolic/glass honeycomb substrate panel with 0.02 in. $(0.508 \mathrm{~mm})$ faces and a stainless steel substrate with 0.005 in. (0.127 mm) faces and phenolic/glass core. The ablator thicknesses are defined in Table 27. The analysis was based on the following assumptions:

(1) Residual stresses at room temperature were caused by the difference in coefficients of thermal expansion between the ablator and substrate and the change in temperature from the bonding or cure temperature to room temperature. (Bond temperature for LDNP $=350^{\circ} \mathrm{F}$ or $450^{\circ} \mathrm{K}$ and cure temperature for NASA $602=230^{\circ} \mathrm{F}$ or $383^{\circ} \mathrm{K}$.) Material shrinkage during cure was not accounted for.

(2) It was assumed that the composite panel remained flat with no slippage at the ablator/substrate interface and no slippage occurred between the honeycomb panel faces.

The re-entry and cold soak problems were analyzed with flexible supports in conjunction with the steel honeycomb panels and with both rigid and flexible supports in the phenolic/glass panel cases. In the case of the rigid supports, it was considered that the 0.05 in $(1.27 \mathrm{~mm})$ aluminum skin acted integrally with the substrate panel and ablator. For the flexible support problems, the aluminum structure was omitted from the analysis. The thermal stress analysis was based on the following relationships:

$$
\sigma_{\text {th }_{n}}=\left(\frac{\mathrm{E}}{1-\mu}\right)_{\mathrm{n}}\left[\epsilon-\alpha_{\mathrm{n}} \Delta \mathrm{T}_{\mathrm{n}}\right]
$$

where

$$
\epsilon=\frac{\Sigma\left(\frac{E A}{1-\mu}\right)_{n} \alpha_{n} \Delta T_{n}}{\Sigma\left(\frac{E A}{1-\mu}\right)_{n}}
$$

The subscript $(n)$ pertains to individual elements comprising the composite system. Ablator mechanical properties are based on Figures 17 and 21 and ref. 13. The orbital thermal environment presented in ref. 3 was used as a basis for determining the pertinent orbital cold soak temperature distributions. Analysis revealed that the cold soak temperature distributions at steady state were the same for both ablator systems and depended only on the material thicknesses. Table 33 shows significant temperatures used in the analysis. 
TABLE 33. COLD SOAK TEMPERATURE DISTRIBUTIONS

\begin{tabular}{|l|c|c|c|c|c|c|}
\hline \multirow{2}{*}{ Panel } & \multicolumn{2}{|c|}{$\begin{array}{c}\text { Ablator } \\
\text { surface }\end{array}$} & \multicolumn{2}{|c|}{$\begin{array}{c}\text { Cold soak te mperature } \\
\text { outer face }\end{array}$} & \multicolumn{2}{c|}{$\begin{array}{l}\text { Substrate } \\
\text { inner face }\end{array}$} \\
\cline { 2 - 7 } & ${ }^{\circ} \mathrm{F}$ & ${ }^{\circ} \mathrm{F}$ & ${ }^{\circ} \mathrm{F}$ & ${ }^{\circ} \mathrm{K}$ & ${ }^{\circ} \mathrm{F}$ & ${ }^{\circ} \mathrm{K}$ \\
\hline $\begin{array}{l}\text { Leading } \\
\text { edge } \\
\text { Crown }\end{array}$ & -72 & 215 & -57 & 224 & -27 & 241 \\
& -80 & 211 & -75 & 214 & -51 & 227 \\
\hline
\end{tabular}

Analysis of the undershoot re-entry after orbital cold soak revealed this condition created less critical thermal stresses than those associated with orbital cold soak. No safety factors were used in the thermal stress analysis. Table 34 summarizes the calculated thermal strains for the room temperature residual and orbital cold soak cases combined.

TABLE 34. ABLATOR THERMAL STRAIN COMPARISONS

\begin{tabular}{|c|c|c|c|c|}
\hline \multirow[b]{2}{*}{$\begin{array}{l}\text { Substrate / } \\
\text { support }\end{array}$} & \multirow[b]{2}{*}{$\begin{array}{l}\text { Ablator / } \\
\text { panel }\end{array}$} & \multicolumn{2}{|c|}{$\begin{array}{l}\text { Thermal strain } \\
\text { at ablator surface }\end{array}$} & \multirow[b]{2}{*}{$\begin{array}{l}\text { Failure } \\
\text { indicatec }\end{array}$} \\
\hline & & $\begin{array}{l}\text { Calculated } \\
\text { strain }\end{array}$ & $\begin{array}{l}\text { Allow able } \\
\text { strain }\end{array}$ & \\
\hline \multirow{4}{*}{$\begin{array}{l}\text { Stainless } \\
\text { steel/ } \\
\text { flexible }\end{array}$} & LDNP/crown & 0.01011 & 0.009 & Yes \\
\hline & $\begin{array}{l}\text { LDNP/leading } \\
\text { edge }\end{array}$ & 0.00735 & 0.009 & No \\
\hline & $\begin{array}{l}\text { NASA } 602 / \\
\text { crown }\end{array}$ & 0.02245 & 0.031 & No \\
\hline & $\begin{array}{l}\text { NASA } 602 / \\
\text { leading edge }\end{array}$ & 0.02106 & 0.031 & No \\
\hline \multirow{4}{*}{$\begin{array}{l}\text { Plastic } \\
\text { laminate / } \\
\text { flexible }\end{array}$} & LDNP/crown & 0.00958 & 0.009 & Yes \\
\hline & $\begin{array}{l}\text { LDNP / leading } \\
\text { edge }\end{array}$ & 0.00592 & 0.009 & No \\
\hline & $\begin{array}{l}\text { NASA } 602 / \\
\text { crown }\end{array}$ & 0.02217 & 0.031 & No \\
\hline & $\begin{array}{l}\text { NASA } 602 / \\
\text { leading edge }\end{array}$ & 0.02003 & 0.031 & No \\
\hline \multirow{4}{*}{$\begin{array}{l}\text { Plastic } \\
\text { laminate / } \\
\text { rigid }\end{array}$} & LDNP/crown & 0.01089 & 0.009 & Yes \\
\hline & $\begin{array}{l}\text { LDNP/leading } \\
\text { edge }\end{array}$ & 0.00747 & 0.009 & No \\
\hline & $\begin{array}{l}\text { NASA } 602 / \\
\text { crown }\end{array}$ & 0.02369 & 0.031 & No \\
\hline & $\begin{array}{l}\text { NASA } 602 / \\
\text { leading edge }\end{array}$ & 0.02285 & 0.031 & No \\
\hline
\end{tabular}


Although the NASA 602 has a higher coefficient of thermal expansion than the LDNP, its stress-strain relationships as indicated in ref. 13 are more favorable. In the case of either material, the nonlinearity of the stress-strain curve prevents the actual thermal stress from being as high as calculated by use of the linear elastic equations cited above. With a complete set of reliable stress-strain curves, a strain analysis could be conducted which would more accurately predict the thermal stresses and strains. The values shown in Table 34 are only as good as the material property data upon which they are based and indicate that the orbital cold soak compatibility problem is best resolved by element testing.

\section{Fabrication and Cost Data Comparisons}

Fabrication problems and processes. - Fabrication processes for the NASA 602 and LDNP ablator materials and phenolic/glass honeycomb substrate panels are defined in Figures 115 and 116 . The use of a steel face substrate panel would require additional steps for splicing, forming, trimming and heat treating the face sheets prior to adhesive bonding the panel assembly. An additional set of tools would be required for the stretch forming operation.

With regard to the stretch forming of the steel faces, the use of a $0.005-i n .(0.127 \mathrm{~mm})$ thick sheet would require development work for curvatures characteristic of most of the vehicle surface. For the curvatures associated with the leading edge panel the minimum acceptable gage would be $0.010 \mathrm{in} .(0.254 \mathrm{~mm})$. In addition, the selection of a sheet splicing method would be influenced by the forming requirements. Accordingly, it can be seen that the selection of 0.005 in. $(0.127 \mathrm{~mm})$ minimum gage for the stainless steel structural optimization studies is somewhat optimistic.

The nature of honeycomb panel fabrication methods is such that close tolerance assemblies can be turned out with a high degree of repeatability. Analysis has shown the overall heat shield performance to be insensitive to moderate changes in the substrate panel depth. Of more significance is the final thickness of the ablator material. Once the LDNP molding tools are built (and material shrinkage is accounted for), the LDNP ablator slabs can be turned out with a high degree of repeatability, and thickness toler ances will be as good as the basic tool will permit. To achieve final thicknesses with the NASA 602 ablator, a surface trimming process is required subsequent to installation in the honeycomb supporting matrix. The final thickness of the elastomeric ablator, then, is dependent upon the accuracy with which it can be determined by available quality control techniques. Experience with such methods as eddy current and needle probe devices indicates that the ablator thickness can be measured within $\pm 2 \%$. 
It would be expected that, due to the nature of the material and the mode of installation, relatively large thickness toler ances would be realized for the microquartz insulation system. The only significant effect on the heat shield performance would be a change in the structure temperature since calculations have shown the bondline temperature to be relatively insensitive to insulation thickness changes. The best cure for this problem would be the imposition of rigid quality control standards during installation and the inclusion of the insulation toler ance problem in the development of heat shield safety factors.

Other fabrication problems related to the thermostructural panel concepts are discussed in related sections of this report and are briefly reviewed below.

(1) Warpage of the composite panel in any combination of ablators and substrate materials will be a significant problem, particularly in the panel sizes proposed herein. It is possible that development work in this area (which is a definite requirement) would even show this problem to be a panel size constraint factor. The warpage could be removed by tying the panel to the vehicle structure by a multipost support system, but this only aggravates the residual stress problem that contributed to the warpage in the first place.

(2) The significant drawback to the LDNP from a fabrication point of view is the matching of the ablator/substrate interface. Quality control, fabrication, cost, and, ultimately, reliability are areas that would be penalized by the use of this material within current state-ofthe-art techniques. One possible solution to this problem could be the development of a castable LDNP and honeycomb matrix composite similar to that employed with the elastomeric systems.

(3) Current application techniques for the elastomeric ablators rely on filling each cell of the supporting matrix individually. The method, though time consuming, is effective in minimizing voids and assuring the best possible bond to the cell walls. Future development efforts could result in more efficient application techniques for this material, such as filling complete panels by vacuum bag techniques.

Fabrication cost breakdown. - A detailed appraisal of the cost factors associated with the candidate thermostructural panel concepts was performed. The cost analysis was based on the same model as was used for the preliminary cost comparisons (see Material and Fabrication Cost Comparisons). Overheads, general and administrative burden and fee were included in the cost analysis. Table 35 presents the final cost comparison data. These cost figures are slightly higher than the preliminary values quoted in Table 13 and reflect a more detailed costing of tooling requirements, particularly for the steel stretch forming. 
TABLE 35. THERMOSTRUCTURAL PANEL COST COMPARISONS

\begin{tabular}{|c|c|c|c|c|c|}
\hline \multirow{2}{*}{$\begin{array}{c}\text { Ablator } \\
\text { material }\end{array}$} & \multirow[b]{2}{*}{ Item } & \multicolumn{2}{|c|}{$\begin{array}{c}\text { Plastic laminate } \\
\text { honeycomb substrate }\end{array}$} & \multicolumn{2}{|c|}{$\begin{array}{c}\text { Steel face/plastic } \\
\text { laminate core } \\
\text { honeycomb substrate }\end{array}$} \\
\hline & & $\$ / \mathrm{ft}^{2}$ & $\$ / \mathrm{m}^{2}$ & $\$ / \mathrm{ft}^{2}$ & $\$ / \mathrm{m}^{2}$ \\
\hline \multirow[t]{8}{*}{ NASA 602} & $\begin{array}{l}\text { Manufacturing } \\
\text { labor }\end{array}$ & 208 & 2,238 & 196 & 2,109 \\
\hline & $\begin{array}{l}\text { Tooling } \\
\text { labor }\end{array}$ & 304 & 3,271 & 353 & 3,798 \\
\hline & $\begin{array}{l}\text { Quality } \\
\text { control }\end{array}$ & 78 & 839 & 83 & 893 \\
\hline & $\begin{array}{l}\text { Total } \\
\text { labor }\end{array}$ & 590 & 6,348 & 632 & 6,800 \\
\hline & $\begin{array}{l}\text { Manufacturing } \\
\text { material }\end{array}$ & 98 & 1,054 & 99 & 1,065 \\
\hline & $\begin{array}{l}\text { Tool } \\
\text { material }\end{array}$ & 22 & 237 & 33 & 355 \\
\hline & $\begin{array}{l}\text { Total } \\
\text { material }\end{array}$ & 120 & 1,291 & 132 & 1,420 \\
\hline & $\begin{array}{l}\text { Gr and } \\
\text { total }\end{array}$ & 710 & 7,639 & 764 & 8,220 \\
\hline \multirow{8}{*}{$\begin{array}{l}\text { Low } \\
\text { density } \\
\text { nylon } \\
\text { phenolic }\end{array}$} & $\begin{array}{l}\text { Manufacturing } \\
\text { labor }\end{array}$ & 155 & 1,668 & 142 & 1,528 \\
\hline & $\begin{array}{l}\text { Tooling } \\
\text { labor }\end{array}$ & 677 & 7,285 & 724 & 7,790 \\
\hline & $\begin{array}{l}\text { Quality } \\
\text { control }\end{array}$ & 119 & 1,280 & 121 & 1,302 \\
\hline & $\begin{array}{l}\text { Total } \\
\text { labor }\end{array}$ & 951 & 10,233 & 987 & 10,620 \\
\hline & $\begin{array}{l}\text { Manufacturing } \\
\text { material }\end{array}$ & 122 & 1,313 & 127 & 1,367 \\
\hline & $\begin{array}{l}\text { Tool } \\
\text { material }\end{array}$ & 65 & 699 & 68 & 732 \\
\hline & $\begin{array}{l}\text { Total } \\
\text { material }\end{array}$ & 187 & 2,012 & 195 & 2,099 \\
\hline & $\begin{array}{l}\text { Grand } \\
\text { total }\end{array}$ & 1138 & 12,245 & 1182 & 12,719 \\
\hline
\end{tabular}


Note that a small cost difference exists between the steel and plastic laminate substrate configurations. However, by changing from a NASA 602 ablator to LDNP, the cost is increased by approximately $60 \%$. In terms of the total vehicle, the costs can be estimated based on the wetted area of $829 \mathrm{ft}^{2}\left(77.01 \mathrm{~m}^{2}\right)$. For a phenolic/glass laminate substrate panel, the total heat shield system would cost $\$ 588,000$ per vehicle with the NASA 602 ablator and $\$ 940,000$ per vehicle for the LDNP ablator. The total cost differential is $\$ 352,000$ per vehicle in favor of the NASA 602 elastomeric ablator.

\section{Vehicle Heat Shield Weight Comparisons}

Heat shield weight analysis program and safety factor criteria. An existing 1620 computer program was utilized in order to establish the relative total heat shield weights between the NASA 602 and LDNP systems. Basically, the program functions by breaking the vehicle surface down into a group of finite areas, assigning a stagnation point heating rate ratio to each area, referring to an appropriate heat shield weight design curve and subsequently integrating the weights over the complete vehicle. An option is provided for the inclusion of safety factors on heating and material thickness.

The total heat shield system weights were computed for a 26.67-ft $(8.128-\mathrm{m})$ reference length $\mathrm{HL}-10$ lifting entry vehicle configuration with no canopy. For purposes of analysis, it was assumed that the substrate structure was an all phenolic/glass laminate honeycomb panel of $0.375 \mathrm{in} .(9.52 \mathrm{~mm})$ depth and $0.02 \mathrm{in} .(0.508 \mathrm{~mm})$ faces. Heat shield design safety factors employed here are based on ref. 15. For windward vehicle surfaces, a factor of 1.15 was applied to the heating rates. The corresponding factor for leeward surfaces was 1.50. A factor of 1.10 was applied to all calculated ablator and insulation thicknesses. Runs were performed both with and without safety factors in order to determine the influence of safety factors on heat shield weights. It should also be noted that the heat shield weights are composites of either the overshoot or nominal trajectory, whichever is design critical at any location.

Heat shield weight comparisons.- Table 36 presents the heat shield weight comparisons.

TABLE 36. HEAT SHIELD SYSTEM WEIGHT COMPARISONS

\begin{tabular}{|c|c|c|c|c|c|c|c|c|c|}
\hline \multirow{2}{*}{$\begin{array}{l}\text { Ablator } \\
\text { system }\end{array}$} & \multicolumn{2}{|c|}{$\begin{array}{c}\text { Insulation } \\
\text { weight }\end{array}$} & \multicolumn{2}{|c|}{$\begin{array}{c}\text { Ablator } \\
\text { weight }\end{array}$} & \multicolumn{2}{|c|}{$\begin{array}{l}\text { Substrate } \\
\text { weight }\end{array}$} & \multicolumn{2}{|c|}{$\begin{array}{c}\text { Total } \\
\text { weight }\end{array}$} & \multirow{2}{*}{$\begin{array}{l}\text { Safety } \\
\text { factors }\end{array}$} \\
\hline & lb & $\mathrm{kg}$ & lb & $\mathrm{kg}$ & $1 b$ & $\mathrm{~kg}$ & $1 \mathrm{~b}$ & $\mathrm{~kg}$ & \\
\hline NASA 602 & 754.8 & 342.4 & 1752 & 794.7 & 812.4 & 368.5 & 3319.2 & 1505.6 & Yes \\
\hline LDNP & 778.7 & 353.2 & 1418.2 & 643.3 & 854 & 387.4 & 3050.9 & 1383.9 & Yes \\
\hline NASA 602 & 686.2 & 311.3 & 1343.3 & 609.3 & 812.4 & 368.5 & 2841.9 & 1289.0 & No \\
\hline LDNP & 707.9 & 321.1 & 1086.1 & 492.7 & 854 & 387.4 & 2648 & 1201.1 & No \\
\hline
\end{tabular}


The LDNP heat shield system is $268.3 \mathrm{lb}(121.7 \mathrm{~kg})$ lighter than the NASA 602 heat shield, which represents a weight saving of $8 \%$. It should be noted that these weights are based on the substrate panel being designed at its optimum weight for the panel depth of 0.375 in. $(9.52 \mathrm{~mm})$. As was previously shown in the section on Application of Design Data, this means that the LDNP system requires approximately four times as many supports as the NASA 602 system. The relative substrate panel weights in Table 36 include the weight effect of the different support requirements. This means then, that the $8 \%$ weight advantage of the LDNP system can be realized only if the greater number of supports is used. Data presented in the section on Application of Design Data also show that for the same number of supports, the weight of the LDNP system substrate panel is $165 \mathrm{lb}(74.8 \mathrm{~kg})$ greater than that of the NASA 602 system substrate. This boosts the weight of the LDNP heat shield system to $3174.3 \mathrm{lb}(1439.9 \mathrm{~kg})$ which corresponds to a weight saving with the LDNP system of $145 \mathrm{lb}(65.8 \mathrm{~kg})$ or $4.4 \%$.

The use of the heat shield safety factors employed in this study increases the NASA 602 system weight by $477 \mathrm{lb}(216.4 \mathrm{~kg})$ or $17 \%$ and the LDNP system weight by $403 \mathrm{lb}(182.8 \mathrm{~kg})$ or $15 \%$.

It may be concluded on the basis of this analysis that the LDNP system offers a weight advantage.

\section{Selection of Optimum Thermostructural Panel Concept}

Based on the studies and comparisons generated in this investigation, a recommendation can be made for an optimum refurbishable composite thermostructural panel concept for manned lifting entry vehicle application.

Ablator materials. - Table 37 summarizes the significant comparisons between the NASA 602 and LDNP systems.

TABLE 37. COMPARISON OF ABLATOR CAPABILITIES

\begin{tabular}{|l|l|l|l|}
\hline \multicolumn{1}{|c|}{ Factor } & NASA 602 & LDNP & \multicolumn{1}{c|}{ Remarks } \\
\hline $\begin{array}{l}\text { Total heat } \\
\text { shield } \\
\text { weight }\end{array}$ & $3319 \mathrm{lb}$ & $3051 \mathrm{lb}$ & $\begin{array}{l}\text { LDNP system re- } \\
\text { quires four times } \\
\text { number of NASA } 602\end{array}$ \\
& $\begin{array}{l}1384 \mathrm{~kg} \\
\text { system supports }\end{array}$ & $\begin{array}{l}\text { Same number of } \\
\text { supports for each } \\
\text { system. 145 Ib } \\
\text { (66 kg) weight } \\
\text { saving with LDNP }\end{array}$ \\
\hline
\end{tabular}


TABLE 37. - Concluded

\begin{tabular}{|c|c|c|c|}
\hline Factor & NASA 602 & LDNP & Remarks \\
\hline Cost & $\begin{array}{l}\$ 588,000 \text { per } \\
\text { vehicle }\end{array}$ & $\begin{array}{l}\$ 940,000 \text { per } \\
\text { vehicle }\end{array}$ & $\begin{array}{l}\$ 352,000 \text { per ve- } \\
\text { hicle cost saving } \\
\text { with NASA } 602 \\
\text { system }\end{array}$ \\
\hline Fabrication & $\begin{array}{l}\text { Ablator injected } \\
\text { into individual } \\
\text { cells }\end{array}$ & $\begin{array}{l}\text { Critical ablator / } \\
\text { substrate interface } \\
\text { problem. Develop- } \\
\text { ment of fabrication } \\
\text { techniques required }\end{array}$ & $\begin{array}{l}\text { Fewer fabrication } \\
\text { problems with } \\
\text { NASA } 602 \text {. Com- } \\
\text { posite panel warp- } \\
\text { age in large sizes } \\
\text { is a problem with } \\
\text { both ablators. } \\
\text { Less severe with } \\
\text { NASA } 602\end{array}$ \\
\hline $\begin{array}{l}\text { Thermal } \\
\text { stress }\end{array}$ & $\begin{array}{l}\text { Elastomers per- } \\
\text { form well in cold } \\
\text { soak environment }\end{array}$ & $\begin{array}{l}\text { Analysis indicates } \\
\text { LDNP is marginal } \\
\text { for cold soak }\end{array}$ & $\begin{array}{l}\text { Thermal stress } \\
\text { problem less severe } \\
\text { with NASA } 602\end{array}$ \\
\hline Reliability & $\begin{array}{l}\text { Attachment tech- } \\
\text { nique assures } \\
\text { more reliable } \\
\text { bond }\end{array}$ & $\begin{array}{l}\text { More susceptible } \\
\text { to damage. Assur- } \\
\text { ance of bond integ- } \\
\text { rity less certain }\end{array}$ & $\begin{array}{l}\text { NASA } 602 \text { is more } \\
\text { reliable }\end{array}$ \\
\hline Reparability & $\begin{array}{l}\text { Ease of field } \\
\text { repair }\end{array}$ & $\begin{array}{l}\text { Requircs plugs or } \\
\text { development of a } \\
\text { castable material } \\
\text { for repair }\end{array}$ & $\begin{array}{l}\text { Fewer repair prob- } \\
\text { lems with NASA } 602\end{array}$ \\
\hline
\end{tabular}

Based on its overall design applicability (performance, fabrication, and cost), the NASA 602 elastomeric ablator material was selected. The only penalty incurred by this selection is with regard to total heat shield weight, and it is of a relatively small magnitude.

Substrate panel materials. - Table 38 summarizes the pertinent considerations relative to the selection of a substrate panel material. 
TABLE 38. COMPARISON OF SUBSTRATE PANEL MATERIAL CAPABILITIES

\begin{tabular}{|c|c|}
\hline Substrate Panel & Remarks \\
\hline $\begin{array}{l}\text { Phenolic/glass face } \\
\text { and core }\end{array}$ & $\begin{array}{l}\text { (1) Most efficient in terms of thermal and } \\
\text { structural design } \\
\text { (2) } 4 \mathrm{ft}(1.219 \mathrm{~m}) \text { width size constraint with } \\
\text { no splices } \\
\text { (3) No serious fabrication problems }\end{array}$ \\
\hline $\begin{array}{l}\text { Phenyl-silane/glass } \\
\text { face and core }\end{array}$ & $\begin{array}{l}\text { (1) Since low temperature design case is crit- } \\
\text { ical, phenyl-silane/glass is inferior to } \\
\text { phenolic/glass } \\
\text { (2) Slightly higher thermal conductivity than } \\
\text { phenolic/glass }\end{array}$ \\
\hline $\begin{array}{l}\text { Steel face and } \\
\text { phenolic/glass core }\end{array}$ & $\begin{array}{l}\text { (1) Heavier structurally than plastic laminates } \\
\text { (2) Slightly more costly than plastics } \\
\text { (3) Fabrication problems constrain thickness } \\
\text { that can be used. Must be spliced to make } \\
\text { large panels } \\
\text { (4) Requires flexible supports which significantly } \\
\text { (5) Requires more supports than phenolic/glass } \\
\text { panels for optimum weight }\end{array}$ \\
\hline $\begin{array}{l}\text { Plastic laminate or } \\
\text { steel faces with } \\
\text { steel core }\end{array}$ & $\begin{array}{l}\text { (1) Higher thermal conductivity } \\
\text { (2) Lightest core with same cell size as phenolic/ } \\
\text { glass core weighs } 12.8 \mathrm{lb} / \mathrm{ft}^{3}\left(205.1 \mathrm{~kg} / \mathrm{m}^{3}\right) \\
\text { as compared to } 5.5 \mathrm{lb} / \mathrm{ft}^{3}\left(88.1 \frac{\mathrm{kg}}{3}\right) \text { for } \\
\text { plastic laminate. Larger cell sizes in- } \\
\text { crease intercell face stability problem } \\
\text { (3) No structural advantages since panel deflec- } \\
\text { tion is not critical }\end{array}$ \\
\hline
\end{tabular}

The advantage is obviously with the phenolic/glass honeycomb substrate panel configuration since it can be used without compromising the substrate panel performance in any area.

Adhesive bond.- The advantages of selecting HT-424 as the adhesive bond material are:

(1) Excellent high temperature performance

(2) Practical fabrication and cure requirements

(3) High reliability (based on extensive prior usage) 
Insulation. - As a result of comparisons (see Discussion of Candidate Concepts and Materials) and the insulation optimization studies, the insulation material selected was multilayer microquartz. Tests have shown that for the operational temper ature range considered no radiation shields will be required. Due to its hygroscopic nature, the insulation should be applied to the vehicle in sealed moistureproof plastic film bags.

Panel support configuration.- Problems associated with the integration of panel support concepts into the heat shield system have been briefly outlined in various sections of this report. Based on the se considerations and the results of a related study which dealt specifically with this problem (see ref. 14), the panel support concept selected consists of a molded phenolic/glass flanged cup which is bonded to the lower face of the substrate panel.

Structural layout drawings. - Layout drawings have been gener ated for the leading edge and crown panels. Dimensions and materials are based on the design data gener ated during this study. The substrate panel depth selected was $0.375 \mathrm{in} .(9.52 \mathrm{~mm})$ and is considered to be representative of an optimum substrate panel depth. Selection of a true optimum would, of necessity, be predicated upon a consideration of volumetric efficiency/total heat shield thickness and weight interrelationships, which was beyond the scope of this particular task. An additional factor would be the support spacing/panel depth tradeoff, which has been defined herein. The layout drawings are presented in Figures 117 through 119.

\section{PARAMETRIC STUDIES}

An investigation was conducted in order to determine the effect of variations in environmental factors and material properties on the predicted performance of the composite thermostructural panel system.

\section{Variation of Environmental Factors}

Variations in the local heating rate ratio of $\pm 15 \%$ were imposed upon a set of nominal heat shield thicknesses for both the LDNP and NASA 602 ablator systems. The basic trajectory used for these analyses was the overshoot since it offered the broadest range of heating rate ratios (0.70 to 0.04$)$ for the vehicle areas of interest. Thicknesses were based on the design curves for over shoot re-entry at heating rate ratios of 0.70 and 0.04 . The effect on the peak bondline and structure temperatures was determined by analysis on the T-CAP-III program. The results are shown in Figures 120 and 121. Note that the LDNP displays a much greater sensitivity to changes in local heating rate. 
The effect of variations in vehicle $\mathrm{W} / \mathrm{S}$ on the critical heat shield temperatures was determined for a $\pm 20 \%$ factor on the nominal vehicle $\mathrm{W} / \mathrm{S}$ of 50 . It can be shown that a percentage change in vehicle $\mathrm{W} / \mathrm{s}$ can be directly related to a change in local heating rate ratio. In this case, for $a \pm 20 \% \mathrm{~W} / \mathrm{S}$ change, the respective factors on heating rate ratio are 1.08 and 0.911 . Results are shown in Figure 122 for the case of a NASA 602 ablator system and a nominal re-entry. The nominal re-entry was investigated in this case since the effects of variation in heating rate ratio have been previously examined for the overshoot trajectory. Hence, the effects of W/S change can be evaluated in Figures 120, 121 and 122. The nominal heating rate ratios are 0.565 and 0.04 . It is seen that for the case considered, relatively large changes in vehicle $\mathrm{W} / \mathrm{S}$ do not significantly alter the maximum bondline temperatures.

\section{Variation of Material Ablation and Heat Transfer Characteristics}

The effect on critical peak temperatures of variations in a number of significant material thermal properties was evaluated. The nominal heat shield thicknesses used for the study were based on the overshoot re-entry heat shield design curves. The procedure was to maintain all parameters at their nominal values except the one being investigated. In some areas, this practice is open to question, as in the case of variable density where it would be expected that the thermal conductivity of the material would also undergo a change. The parameters studied are listed below:

(1) Ablator thermal conductivity

(2) Ablator density

(3) Char surface recession rates (LDNP only)

(4) Transpiration factor

(5) Char emissivity

Results of the parametric analysis are shown in Figures 123 through 129.

The influence of thermal conductivity and thickness changes in the microquartz insulation system were previously evaluated and are defined in Figure 64.

\section{Variation of Ablator Allowable Strain}

The effect of variations in the allowable ablator strain of $\pm 20 \%$ on the required substrate panel weight were evaluated and found to be negligible for both the LDNP and NASA 602 ablator materials. The changes in allowable ablator strain were compensated for by varying the panel depth. Since this merely requires changing the thickness of 
the honeycomb core, the weight change, as would be expected, was extremely small $( \pm 2 \%)$. The condition checked in this analysis was the critical load case on the leading edge panel which was the maximum load factor abort trajectory.

\section{Weight Comparisons of Various Lifting Entry Vehicle Configurations}

The heat shield design data generated in this investigation were applied to the HL-10, M2-F2 and SV-5 lifting body configurations in an attempt to obtain comparative vehicle heat shield weight data. The basic vehicle sizes for both the HL-10 and M2-F2 vehicles were taken as defined in ref. 16. The SV-5 vehicle size was developed to provide the mission capability on a comparable basis with the HL-10 and M2-F2. All vehicle heat shields were sized to account for crossover effects between the overshoot and nominal trajectories. The trajectories are defined in ref. 3 . Table 39 compares the pertinent vehicle dimensions and total heat shield weights for a NASA 602 system.

TABLE 39. COMPAR ATIVE VEHICLE

HEAT SHIELD WEIGHTS

\begin{tabular}{|c|c|c|c|c|c|c|c|c|}
\hline \multirow[b]{2}{*}{ Vehicle } & \multicolumn{2}{|c|}{$\begin{array}{l}\text { Reference } \\
\text { length }\end{array}$} & \multicolumn{2}{|c|}{ Wetted area } & \multicolumn{2}{|c|}{$\begin{array}{l}\text { Total heat } \\
\text { shield weight }\end{array}$} & \multicolumn{2}{|c|}{ Unit weight } \\
\hline & $\mathrm{ft}$ & $\mathrm{m}$ & $\mathrm{ft}^{2}$ & $\mathrm{~m}^{2}$ & $1 b$ & $\mathrm{~kg}$ & $\mathrm{lb} / \mathrm{ft}^{2}$ & $\mathrm{~kg} / \mathrm{m}^{2}$ \\
\hline $\mathrm{HL}-10$ & 26.67 & 8.128 & 829 & 77.01 & 3319 & 1505 & 4.00 & 19.52 \\
\hline $\mathrm{M} 2-\mathrm{F} 2$ & 29.25 & 8.915 & 1183 & 109.9 & 4306 & 1953 & 3.64 & 17.76 \\
\hline $\mathrm{SV}-5$ & 26.11 & 7.958 & 838 & 77.85 & 3416 & 1549 & 4.08 & 19.91 \\
\hline
\end{tabular}

The heat shield weights are based on the safety factors defined under Heat Shield Weight Comparisons. It should be noted that the heat $r$ ate distributions for the M2-F2 vehicle are based on the best available data, but they are considered less reliable than the corresponding values for the HL-10 and SV-5.

\section{DESIGN DEVELOPMENT PROBLEM AREAS AND CONCEPT VERIFICATION TESTING}

\section{Design Development Problem Areas}

Before a reliable thermostructural composite panel design can achieve an operational status, several problem areas must be explored and resolved. In the course of this investigation, significant problem areas were uncovered and will be defined in this section. 
Material property determination. - Thermochemical, thermal and mechanical property prediction for ablative materials is extremely difficult due to the nature of the materials and their behavior at elevated temperature. Available data display large variations and, in general, are quite inconsistent. The solution to the problem lies in three major areas: testing for material properties, design philosophy and quality control. The need for extensive material testing, based on a rigidly standardized set of test procedures (methods, instrumentation, facilities), is clearly indicated, coupled with statistical data reduction techniques. Specimens should be fabricated under conditions that are most nearly identical with those associated with full-scale production components. Application of the se data to the design analysis will require the consideration of material property confidence levels and their influence on the overall heat shield and structure safety factor philosophy. Subsequent to the design phase, the natural follow-on would be the development and imposition of rigid quality control standards during fabrication. A similar problem is associated with the quartz fiber insulation systems in that a firmer definition of their sensitivity to mechanical and atmospheric pressure variations will be required.

Joint design.- The development of a reliable joint design is basic to the overall thermostructural panel design integrity. Preliminary analyses conducted in this investigation indicate that the joint design is not critical. However, the analyses did not account for the strength of the bond between the joint material and the ablator and substrate or its thermal performance. Limited testing of joint configurations on related programs indicates that no serious problems exist in the se areas. Verification testing would be required, however, to assure the compatibility of specific joint geometries and materials with predicted environmental factors.

Adhesive bond integrity.- Analytical techniques for predicting adhesive bond stresses are currently being studied but are not sufficiently reliable for design analysis applications. It would be necessary to evaluate the adhesive bond integrity under cold soak and elevated temperature conditions by appropriate test techniques.

Influence of re-entry corridor on heat shield sizing.- Investigations conducted herein indicate that more than one re-entry trajectory can influence heat shield sizing. The problem here is to determine the best design philosophy relative to safety factor application for heat shield design. Obviously, if the heat shield is sized for a nominal re-entry case only, one set of safety factors would apply. On the other hand, designing the heat shield for the critical trajectory unique to each specific vehicle location would have a significant influence on the safety factor criteria. The latter approach appears more desirable.

Volumetric efficiency/heat shield thickness tradeoff.- Heat shield design can be influenced by the internal volume requirements for specific vehicles and missions depending upon the design philosophy 
applied. The most significant inter action occurs if the heat shield thickness must be built inward from the aerodynamic contours of the vehicle. Since volumetric efficiency of lifting entry vehicles is a critical factor, it would appear that a study of the tradeoffs associated between volumetric capacity, heat shield thickness and aerodynamic performance would be of interest. Possibly a compromise could be arrived at whereby the aerodynamic contours could be expanded and at the same time maintain their proper proportions. The degree of tradeoff here would vary depending upon the specific vehicle in question.

Warpage of composite panels. - The warpage of composite panels as a result of bonding and curing temperature cycles is indicated as a problem requiring fabrication development effort. This problem could be particularly significant in the large panel sizes considered in this investigation.

Effects of nonadiabatic structure. - The heat shield sizing studies were based on the assumption that the aluminum structure of the vehicle experienced no heat losses to the interior of the vehicle. Studies of the effect of cooling the aluminum structure with existing vehicle thermal control systems or auxiliary systems could show a significant net weight and heat shield thickness reduction due to reduced insulation requirements.

Repair techniques. - Work has been performed on related programs to develop heat shield repair and patching techniques for elastomeric ablator materials. Some repaired specimens have been exposed to plasma arc testing with good results. The operational use of elastomeric ablators will require further development efforts in this area in order to establish standard repair techniques. The amenability of the elastomeric ablators to repair in a space environment should also be evaluated.

Thermostructural panel optimization. - Further optimization studies can be applied to the thermostructural panel concept selected in this investigation. Areas of potential improvement are listed below.

1) Ablator. - Lower density elastomeric ablators are available and should be investigated relative to their potential for improving ablative efficiency. In addition, the supporting matrix for the ablator can be improved through the continued development of open mesh resin/glass systems which provide reduced density, reduced thermal conductivity and a mechanical interlock between the filled ablator and matrix.

2) Substrate. - The minimum gage of the phenolic/glass laminates could be further reduced by investigating other glass cloth systems. This would involve strength/weight comparisons. Lighter weight cores could be developed, although the overall weight saving here would be quite small. Higher permissible substrate material temperatures would produce a more efficient heat shield. 
3) Adhesive bond. - Thinner and lighter weight HT-424 adhesive films are available although at a cost in strength. This is a worthwhile area for improvement since the weight of the three bond layers presently comprises approximately $50 \%$ of the total substrate panel weight. Higher permissible bond temperatures would result in a more efficient heat shield.

4) Insulation. - Further investigation of the integral insulation/support concept could result in the development of a more efficient concept in terms of the overall design. The potential advantages of such a system are listed below.

(a) Reduction of basic vehicle structural weight. (Fewer hard points for support point load backup.)

(b) Reduction of substrate panel weight.

(c) Large reduction in number of post supports to attach panel to basic vehicle structure and, therefore, fewer attachments to be removed for refurbishment or repair and fewer penetrations of the ablator surface.

(d) Honeycomb core may be readily contoured to provide exact insulation thickness requirements at any location. This cannot be done as precisely with the quartz fiber insulations for obvious reasons.

The honeycomb/aluminum foil concept can be improved to some extent by employing an open weave flexible core configuration.

\section{Concept Verification Testing}

The development of a verification test program and philosophy must be performed within the framework of constraints defined by test objectives, instrumentation requirements and capabilities, test facility availability and capacity, availability of existing related data, and program costs.

Experimental development and verification of a composite thermostructural panel concept requires the examination of the compatibility of the various system components with the critical environmental factors. Table 40 defines a group of significant test objectives for panels of this type. 


\section{TABLE 40. ELEMENT TEST BREAKDOWN FOR COMPOSITE THERMOSTRUCTURAL PANEL DESIGN VERIFICATION}

\begin{tabular}{|c|c|c|}
\hline Test objective & Type of test & $\begin{array}{l}\text { Applicable test } \\
\text { facilities }\end{array}$ \\
\hline $\begin{array}{l}\text { Thermal performance } \\
\text { of composite panel }\end{array}$ & Thermal & Plasma arc, hot gas \\
\hline $\begin{array}{l}\text { Evaluation of panel } \\
\text { support heat short } \\
\text { problem }\end{array}$ & Thermal & $\begin{array}{l}\text { Plasma arc, hot gas } \\
\text { radiant heat }\end{array}$ \\
\hline $\begin{array}{l}\text { Joint design } \\
\text { verification }\end{array}$ & $\begin{array}{l}\text { Thermal and } \\
\text { structural }\end{array}$ & $\begin{array}{l}\text { Plasma arc, hot gas } \\
\text { space chamber, standard } \\
\text { loading machines }\end{array}$ \\
\hline $\begin{array}{l}\text { Thermal stress com- } \\
\text { patibility of com- } \\
\text { posite panel }\end{array}$ & Structural & Space chamber \\
\hline $\begin{array}{l}\text { Structural integrity } \\
\text { of composite panel } \\
\text { at room temperature }\end{array}$ & Structural & $\begin{array}{l}\text { Static test (loading pads or } \\
\text { pressure differential) }\end{array}$ \\
\hline $\begin{array}{l}\text { Structural integrity } \\
\text { of substrate panel } \\
\text { at elevated tem- } \\
\text { perature }\end{array}$ & Structural & $\begin{array}{l}\text { Static test (loading pads or } \\
\text { pressure differential), } \\
\text { radiant heat }\end{array}$ \\
\hline $\begin{array}{l}\text { Strength determina- } \\
\text { tion of panel supports }\end{array}$ & Structural & $\begin{array}{l}\text { Standard elevated tempera- } \\
\text { ture loading machines }\end{array}$ \\
\hline $\begin{array}{l}\text { Compatibility of } \\
\text { composite panel with } \\
\text { vibration and flutter } \\
\text { environment }\end{array}$ & Structural & $\begin{array}{l}\text { Dynamic shaker, acoustic } \\
\text { horns, wind tunnel }\end{array}$ \\
\hline
\end{tabular}

In general, test specimens should be as near in size to their full-scale counterparts as possible in order to minimize the scaling problem. Furthermore, the use of large-scale test panels will also afford a realistic evaluation of the problems associated with fabrication and quality control of large thermostructural composite panels.

Since the requirements associated with thermal scaling are not compatible with those for structural scaling, meaningful data can be obtained only from tests conducted on specimens proportioned to satisfy either thermal or structural test objectives and subsequently tested to those specific conditions. A constraint on the scaling capability exists as a result of the structural optimization studies conducted 
on the honeycomb substrate panel. It was found that the optimum panel weight was coincident with the minimum available thickness for the plastic laminate face materials. Accordingly, since for precise structural scaling all dimensions must be kept proportional to the fullscale measurements, if scaling is imposed on the panel, it will not be possible to maintain proper dimensional relationships. Exclusive of this problem, the minimum practical panel depth from a fabrication capability standpoint is $0.1875 \mathrm{in} .(4.76 \mathrm{~mm})$. Based on a recommended full-scale panel depth of $0.375 \mathrm{in} .(9.52 \mathrm{~mm})$, the minimum permissible scaling ratio would be 0.50 . It may be concluded that scaling to evaluate structural adequacy of the panels would be impractical due to the minimal dimensions of the full-scale configuration. Studies could be conducted to determine the feasibility of testing panels of reduced length and width but with the same thicknesses as the full scale by simulating the appropriate edge boundary conditions.

The size and capacity of existing test facilities such as plasma arc, hot gas, and space chambers must be considered in establishing the size of the test panels.

In conclusion, it would appear that the most meaningful test program for design verification of the thermostructural panel concept would be one in which a series of element tests were conducted on largeor full-scale specimens tailored to a set of specific test objectives such as those in Table 40 .

\section{CONCLUSIONS}

(1) Studies have been conducted relative to the selection of an optimum composite refurbishable thermostructural panel for application to manned lifting entry vehicles. The configuration selected consists of the following components:

(a) NASA 602 elastomeric silicone ablator in a flexible phenolic/ glass honeycomb matrix

(b) Phenolic/glass laminate honeycomb core and faces substrate panel

(c) HT-424 adhe sive bond

(d) Phenolic/glass molded cup-type rigid panel supports

(e) Multilayer microquartz insulation. 
Although low density nylon phenolic ablator offers a slight system weight advantage, it is inferior to the NASA 602 system in terms of reliability, cost, fabricability and cold soak thermal stress compatibility.

The use of a stainless steel honeycomb substrate panel would require more supports than the phenolic/glass honeycomb substrate panel in order to realize its minimum weight potential. Furthermore, the stainless steel panel sizes would be limited to approximately half the size of the phenolic/glass panels. Potentially severe fabrication problems are indicated for compound curved steel panels in the thickness ranges associated with optimum weight. In terms of weight and cost, the stainless steel honeycomb substrate is slightly inferior to the phenolic/ glass substrate honeycomb panel.

(2) Heat shield design studies conducted for a nominal re-entry and re-entry corridor extremes indicate that critical trajectories for heat shield design will vary over the vehicle surface.

(3) The influence of the mode of panel support was found to be a significant constraint on the allowable panel size for the case of steel substrate panels on flexible supports.

(4) The heat short effect attributed to the panel supports was found to be negligible for plastic laminate supports but quite severe for steel supports. The effect of considering the thermal inertia of the backup structure was found to substantially reduce heat short effects.

(5) An integral insulation/support concept was generated and tested. Results show the need for improved insulation capability in order to benefit from the concept's other favorable design features.

(6) A parametric study interrelating insulation thermal conductivity, thickness and peak aluminum structure temperature was performed and resulted in a design curve that, although based on a specific design case, is generally useful for estimating interaction of these parameters in related problems.

(7) Structural optimization studies were conducted on the substrate panel concepts and materials and, in addition to providing data useful in the selection of an optimum concept, resulted in the generation of a set of design curves for application to similar problems. Significant constraints on the substrate panel design were found to be: permissible ablator strain, substrate material strength, honeycomb face stability, minimum material gage and minimum fabricable panel depth.

(8) Based on the available data, it was determined that panel flutter requirements were not critical for design. 
(9) Parametric studies were conducted to evaluate the effects on critical heat shield temperatures of variations in environmental factors and material properties. Significant temperature changes were in response to variations in ablator density, thermal conductivity, transpiration factor, char emissivity and surface recession characteristics and occurred most noticeably at the critical bondline between the ablator and substrate panel. The aluminum vehicle structure temperature was generally insensitive to the se changes but was found to be strongly dependent upon the insulation thermal conductivity and thickness. Conversely, the bondline temperature was found to be generally insensitive to changes in the insulation thermal conductivity and thickness.

(10) The problem of verification testing of the thermostructural panel concept has been examined and recommendations for a test approach have been made. It is indicated, based on the material gages and panel depths recommended for the full-scale substrate panel design, that scaling models for test would be impractical.

Martin Company

Baltimore, Maryland 21203

March 21; 1966 


\section{APPENDIX A}

\section{DESCRIPTION OF MARTIN T-CAP-III THERMO-CHEMICAL ABLATION PROGRAM}

The T-CAP-III digital analysis program considers the ablative layer to consist of a char layer, pyrolysis zone, and virgin ablator material, each of which are characterized by a density profile. Where applicable, a melt layer is superimposed on the char. For general application, the capability of handling an arbitrary composite arrangement of ablator, insulator and internal structure is included. The surface boundary conditions include arbitrary heating functions versus time based on convection and radiant heating histories, heat input due to char combustion, heat blocked by mass addition to the boundary layer, radiative cooling from the hot char surface, and heat absorbed due to vaporization in the melt layer. A program option is also available to describe the surface boundary condition as a temperature-time history. Surface recession resulting from char oxidation or melt layer flow and/or vaporization is computed with a special option available to read in the surface recession rate as a function of heat input rate or surface temperature. The nonablator material properties input data are considered either in the equation form as power series functions of temperature or in curve form as arbitrary functions of temperature. The ablator thermal properties input data are considered in curve form as functions of both temperature and density. The rate of change of the ablative layer density profile and the resulting pyrolysis products mass flow are calculated utilizing reaction kinetics of the ablator with nth order reaction data based on thermogravimetric analysis. 


\section{REFERENCES}

1. Newell, J.: A Study of Thermo-Structural Design Concepts for Lifting Entry Vehicles. NASA CR-240, 1965.

2. Swann, R.; Brewer, W.; and Clark, R.: Effect of Composition and Density on the Ablative Performance of Phenolic Nylon. (Paper presented at VIII National Meeting of the SAMPE.) 1965.

3. Wing, L. D.; and Eilertson, W. H.: Research Studies and Analysis to Define Manned Lifting Entry Flight Environment. NASA CR-639, 1966.

4. Lightweight Thermal Protection System Development. ASD-TDR63-596, Vol. II, 1963.

5. Glaser, P. E. ; et al: Multilayer Insulation. Mech. Eng., Vol. 87, No. 8, August 1965, pp 23-27.

6. Timoshenko, S.: Theory of Plates and Shells. Second Ed., McGrawHill Book Co., Inc., 1959.

7. Kaufman, S. ; and Hall, D. B.: A Digital Program for Static and Dynamic Analysis of Complex Structures. Martin Co. RM-136, 1963.

8. Kordes, E. P.; Tuovila, W.; and Guy, L.: Flutter Research on Skin Panels. NASA TND-451, 1960.

9. Dowell, E.: The Flutter of Multi Bay Panels at High Supersonic Speeds. ASRL-TR 112-1, 1963.

10. Handbook of Structural Stability. Part III, NASA TN 3783, 1957.

11. Flutter Characteristics of Stage I Between Tank Panels on the Titan Lot B Missile. WDDD-M-SR-58-46, 1958.

12. Experimental Studies of Flutter of Buckled Rectangular Panels at Mach Numbers from 1.2 to 3.0 Including Effects of Pressure Differential and of Panel Width-Length Ratio. NASA-TND-833, 1961.

13. Wilson, R. G.: Thermophysical Properties of Six Charring Ablators for $140^{\circ}$ to $700^{\circ} \mathrm{K}$ and Two Chars from $800^{\circ} \mathrm{K}$ to $3000^{\circ} \mathrm{K}$. NASA TN D-2991, 1965. 
14. Rieckmann, R. E.: Research Study to Provide Concepts of Panel Attachment Mechanisms Suitable for Refurbishable Panel Application. NASA CR-640, 1966.

15. Haire, E. T.: Reliability Analysis \& Safety Factor Studies, Structure and Heat Shield, 48 inch SV-5. Martin Co. Rept. CR-90, 1964.

16. Final Report: A Lifting Re-entry Horizontal Landing Type Logistic Spacecraft (Confidential) Contract NAS 9-1689, The Boeing Company, Rept. D2-22921, 1964.

17. Gynn, G. M. ; et al: Elevated and Room Temperature Properties of Conolon 506 Plastic-Glass Fabric Laminate. WADC TR 57-574, 1957.

18. Boller, K. H.: Strength Properties of Reinforced Plastic Laminates at Elevated Temperatures. ASD TR 61-482, 1961.

19. Dolan, C. M.: Study for Development of Elastomeric Thermal Shield Materials. NASA CR-186, 1965.

20. Chapman, A. J.: Effect of Weight, Density, and Heat Load on Thermal Shielding Performance of Phenolic Nylon. NASA TN D-2196, 1964.

21. Lightweight Thermal Protection System Development. AFMLTR-65-26, Vol. II, 1965.

22. NASA RFP A-9758 (FG-17), Exhibit A, February 23, 1965. 


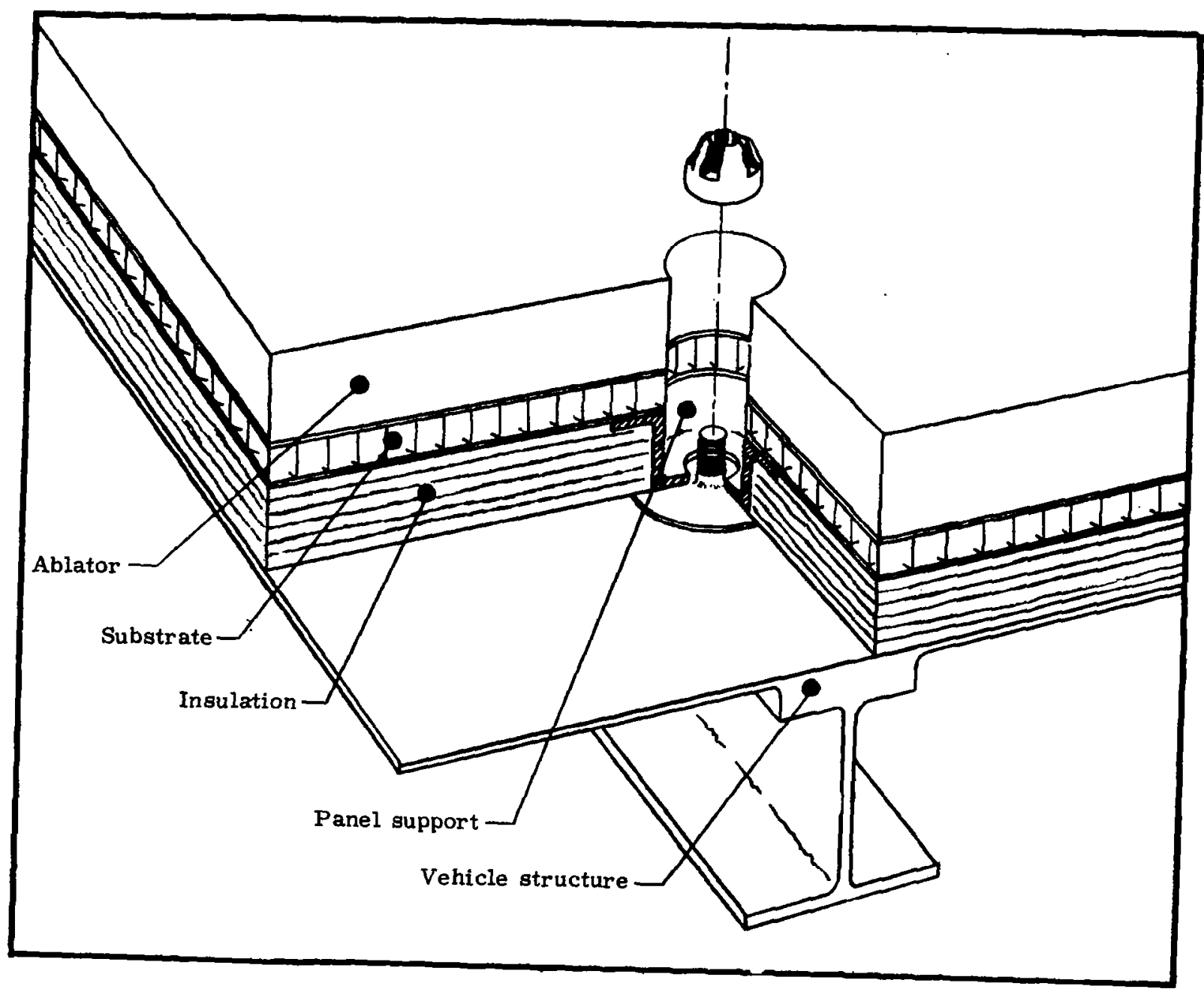

Figure 1, Double Wall Ablative Heat Shield Concept 

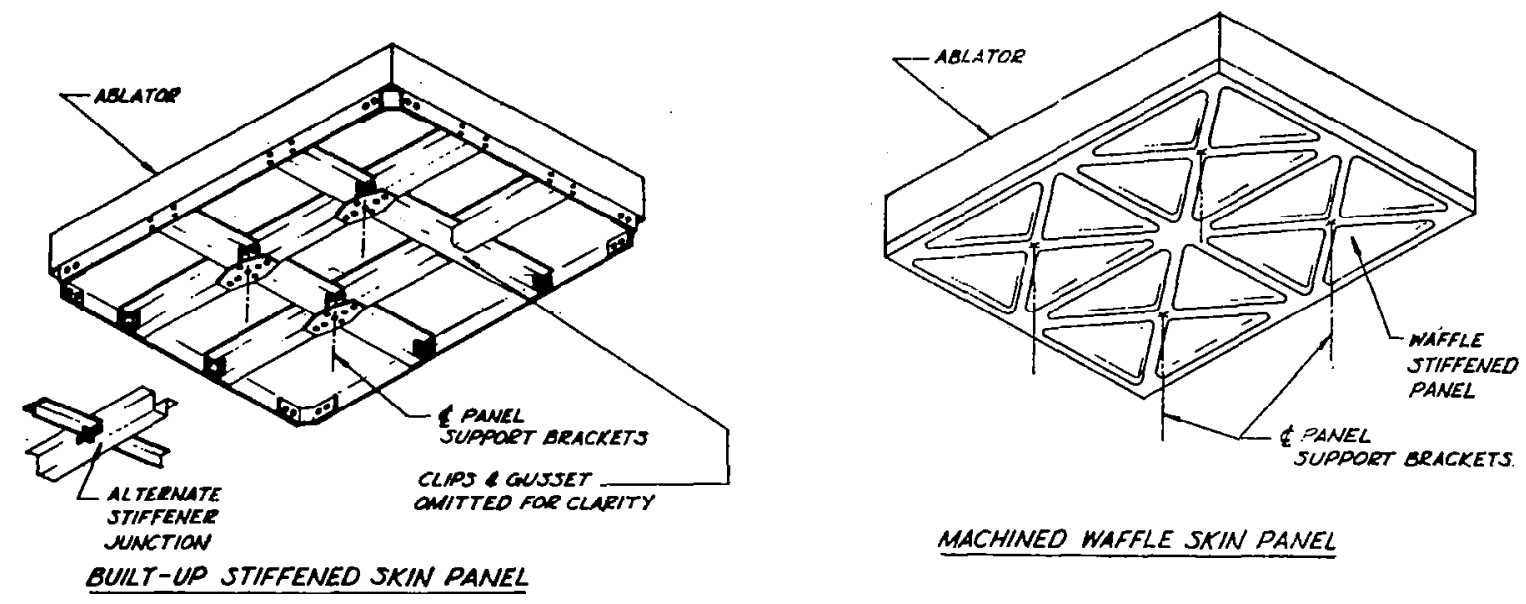

MACHINEO WAFFLE SKIN PANEL

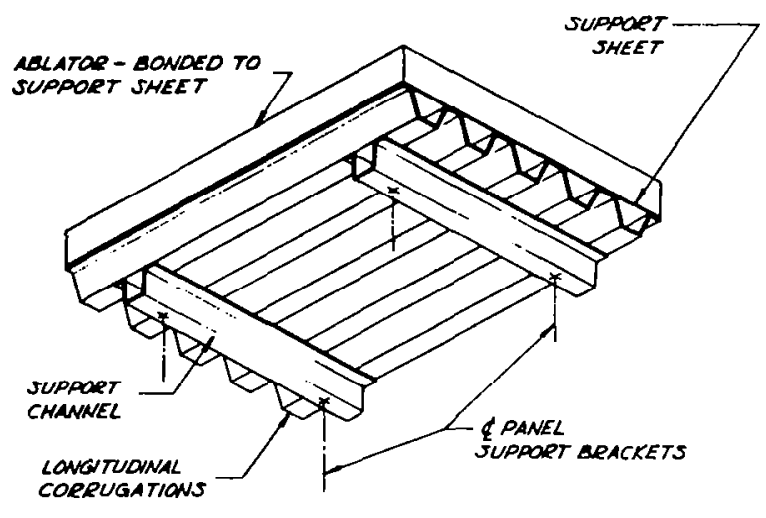

SKIN-CORRUGATION PANEL

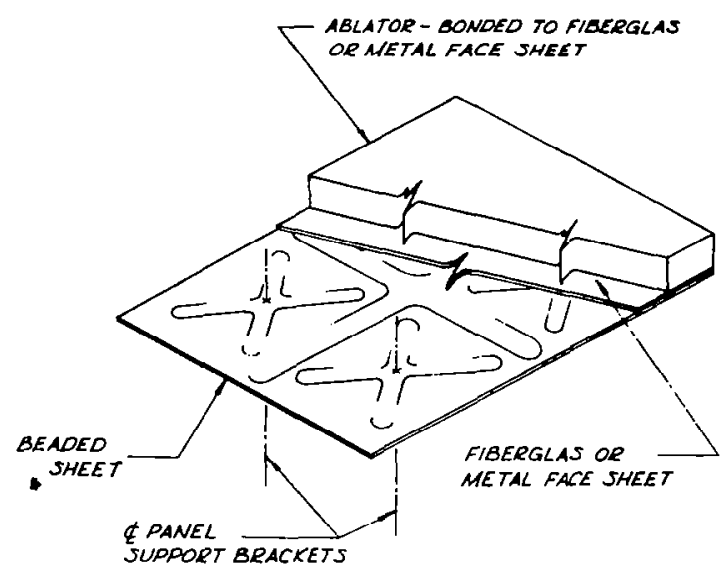

BEAD STIFFENED SKIN PANEL

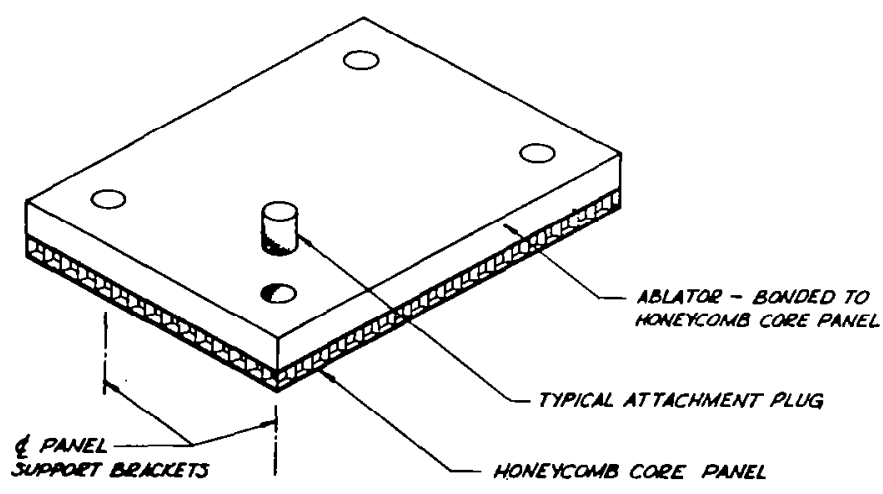

HONEYCOMB PANEL

FIGURE 2. PRELIMINARY SUBSTRATE PANEL CONCEPTS 


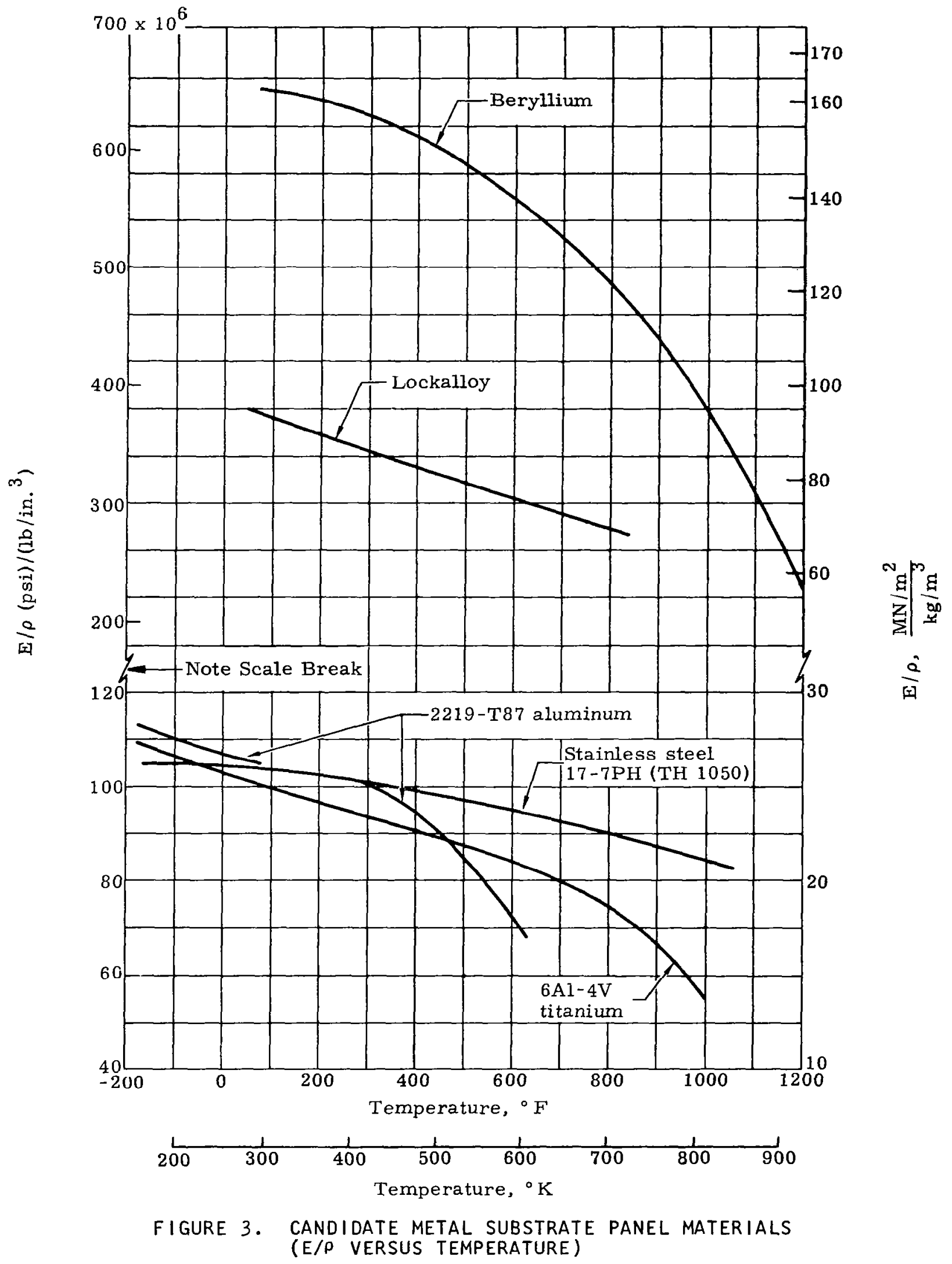




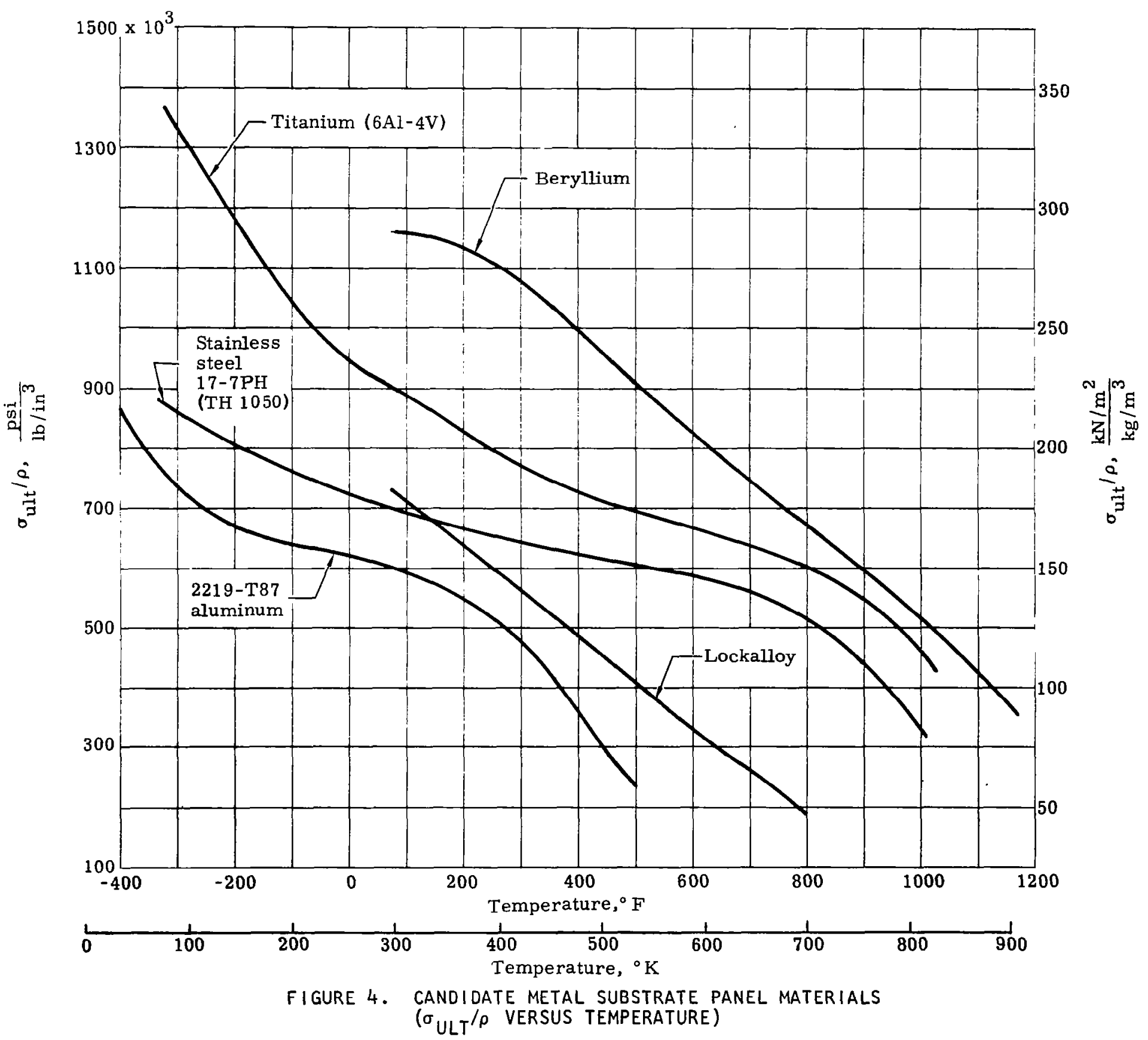




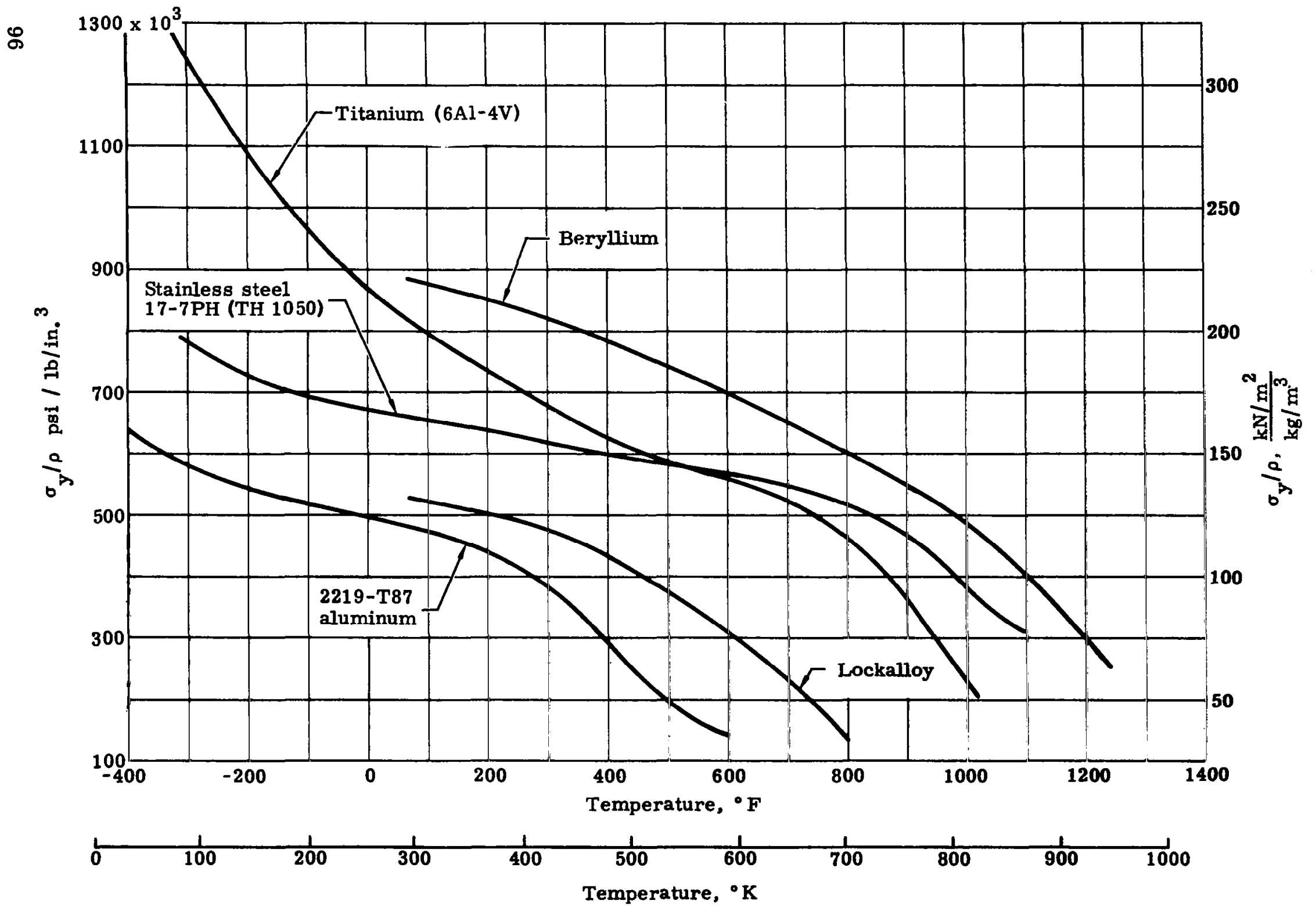

FIGURE 5. CANDIDATE METAL SUBStrate PANEL Materials

( $\sigma_{y} / \rho$ VERSUS TEMPERATURE) 


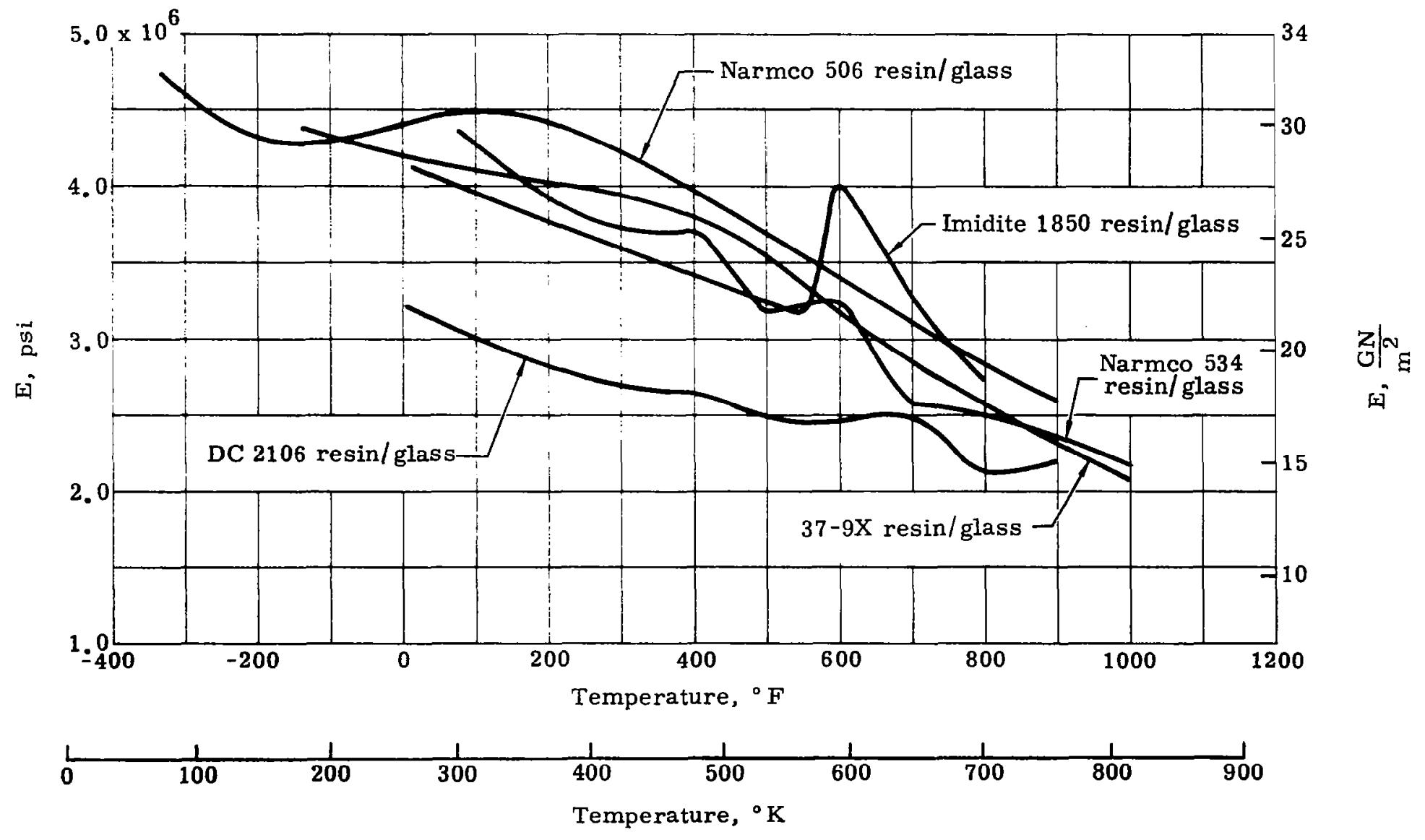

FIGURE 6. CANDIDATE PLASTIC LAMINATE SUBSTRATE PANEL MATERIALS (E VERSUS TEMPERATURE) 


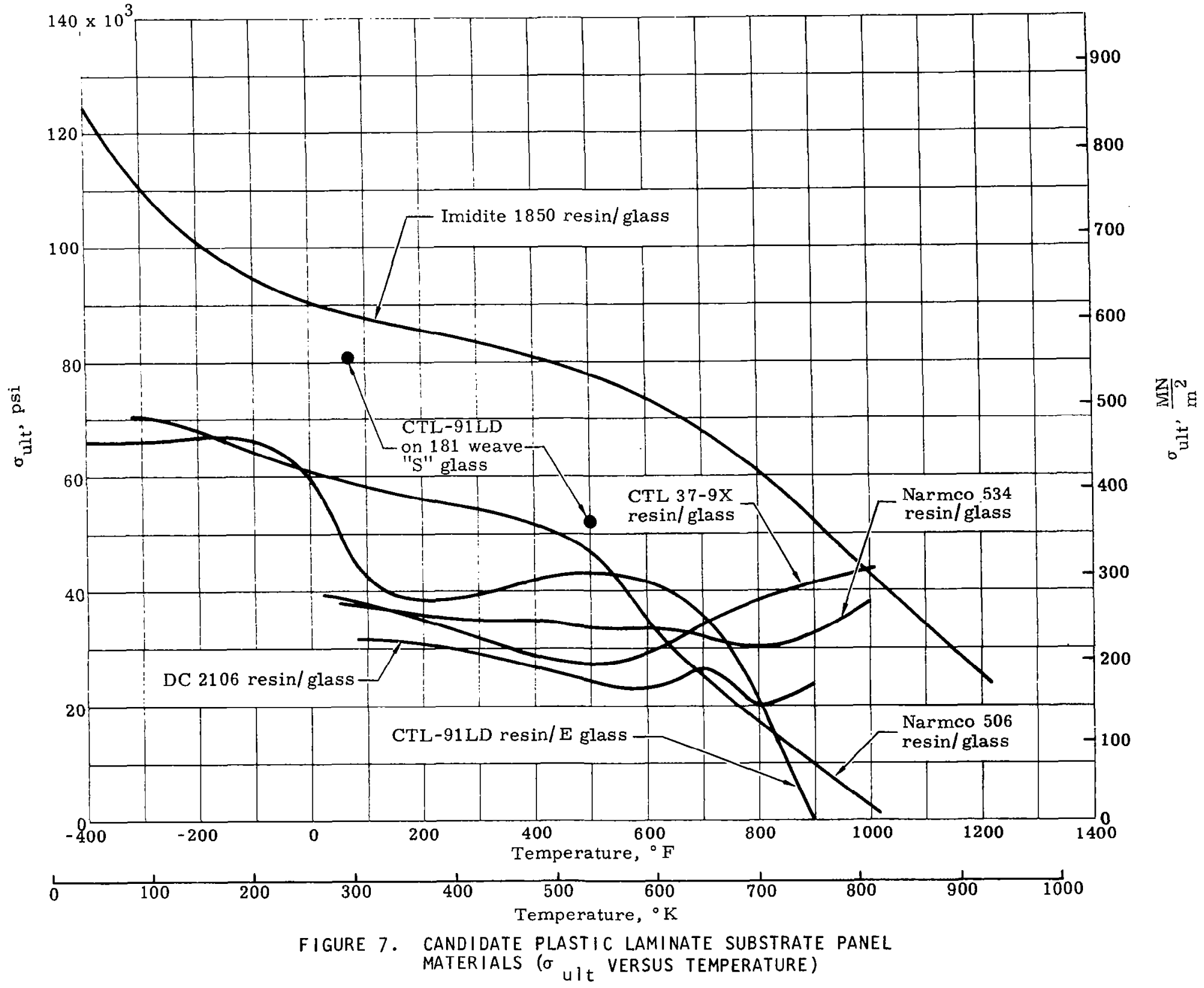



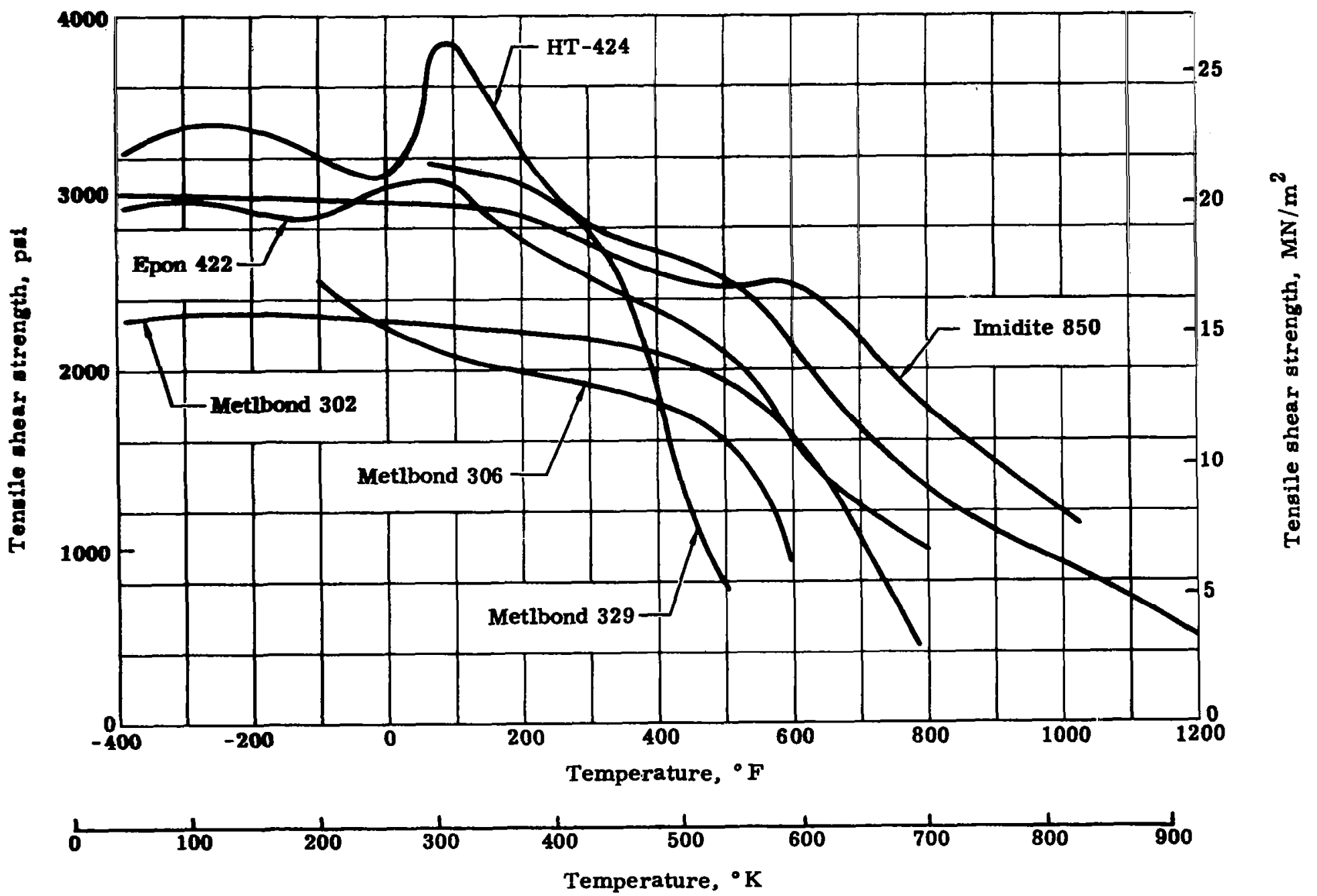

FIGURE 8. CANDIDATE ADHESIVE BOND MATERIALS (TENSILE 


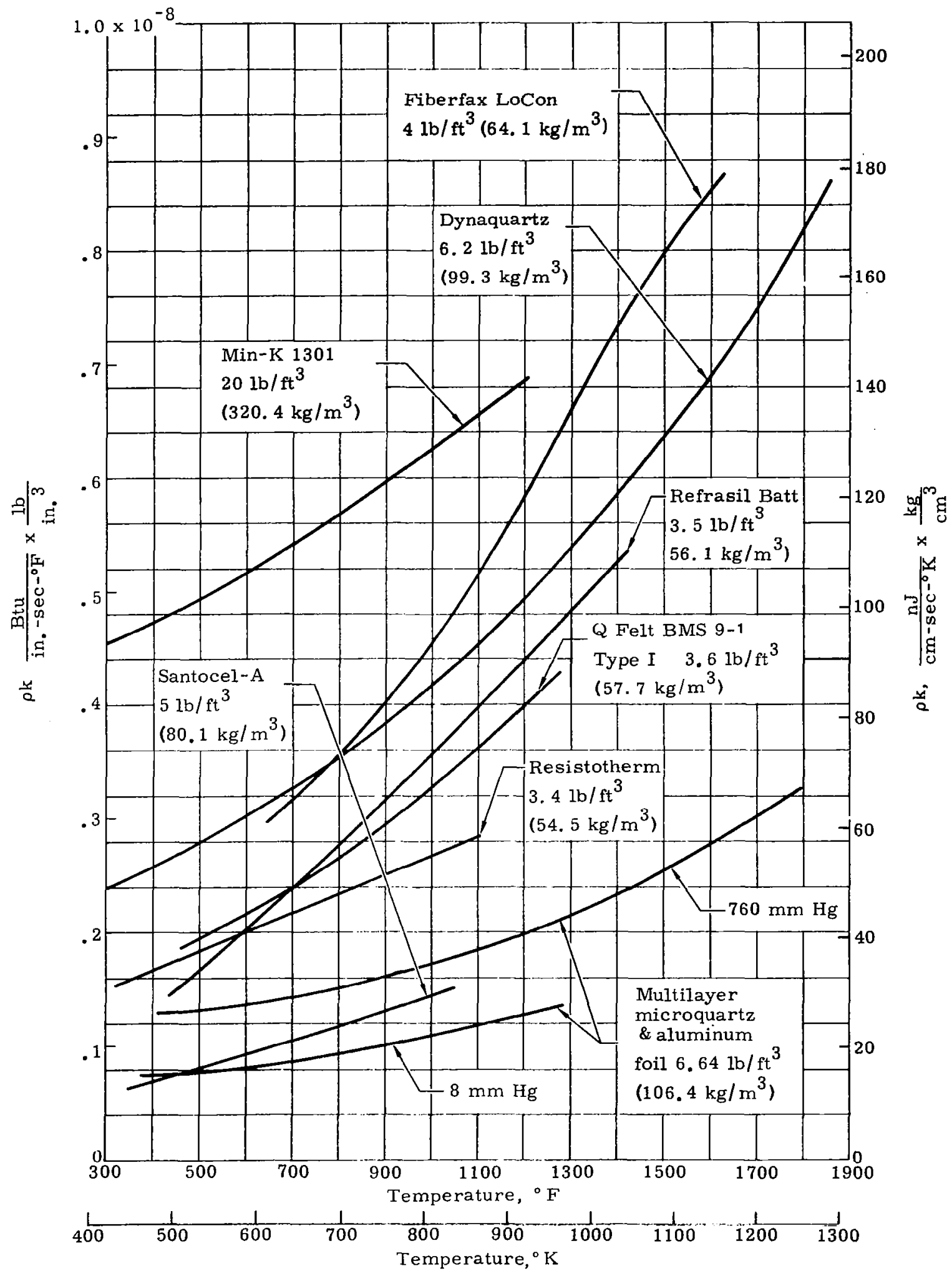

FIGURE 9. CANDIDATE INSULATION MATERIALS ( $\rho_{\mathrm{K}}$ VERSUS TEMPERATURE) (Refs. 4 and 21) 


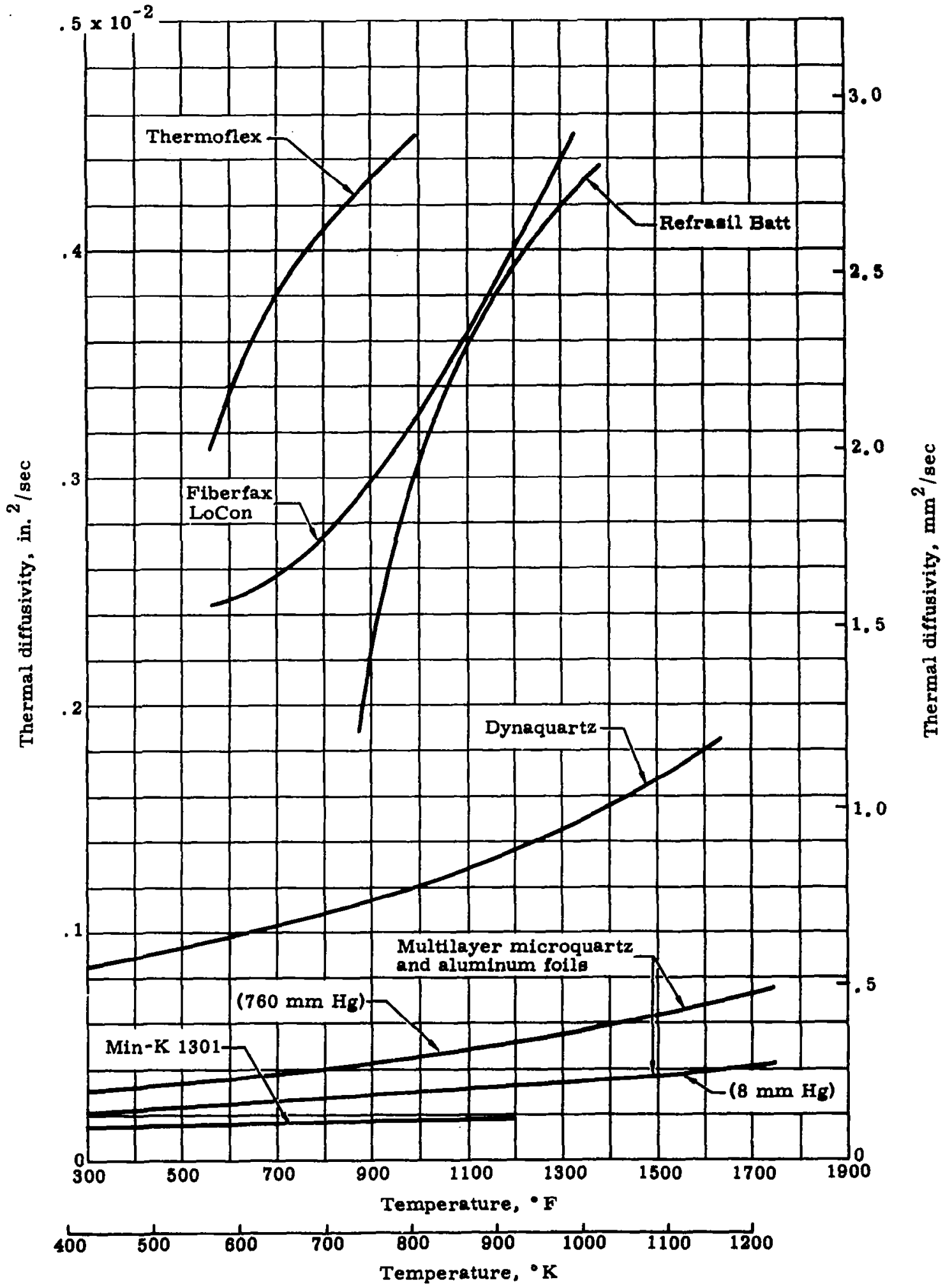

FIGURE 10. CANDIDATE INSULATION MATERIALS (THERMAL DIFFUSIVITY VERSUS TEMPERATURE) (Refs. 4 and 2I) 


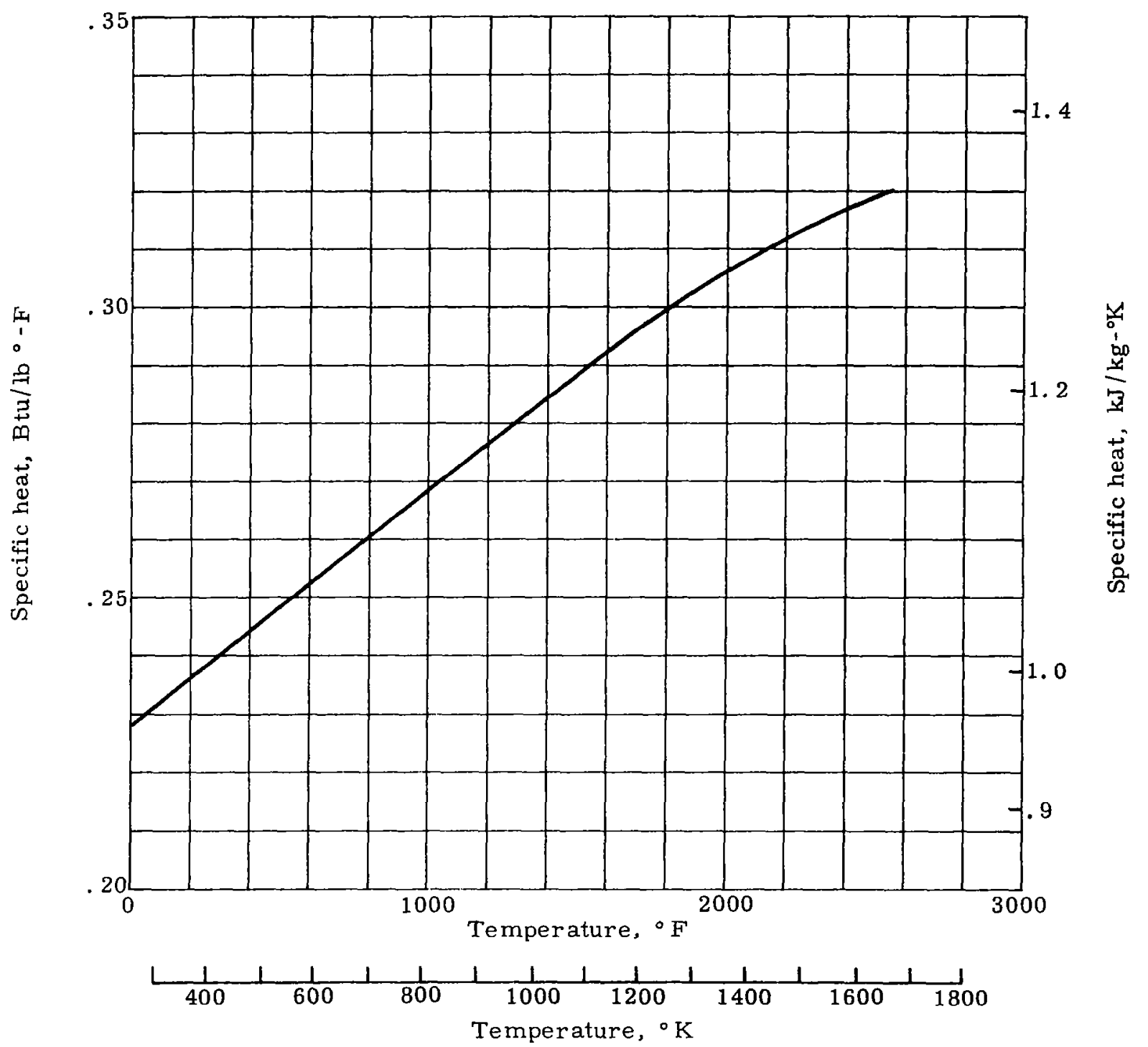

FIGURE II. MULTILAYER MICROQUARTZ SPECIFIC HEAT VERSUS TEMPERATURE (Ref. 4) 


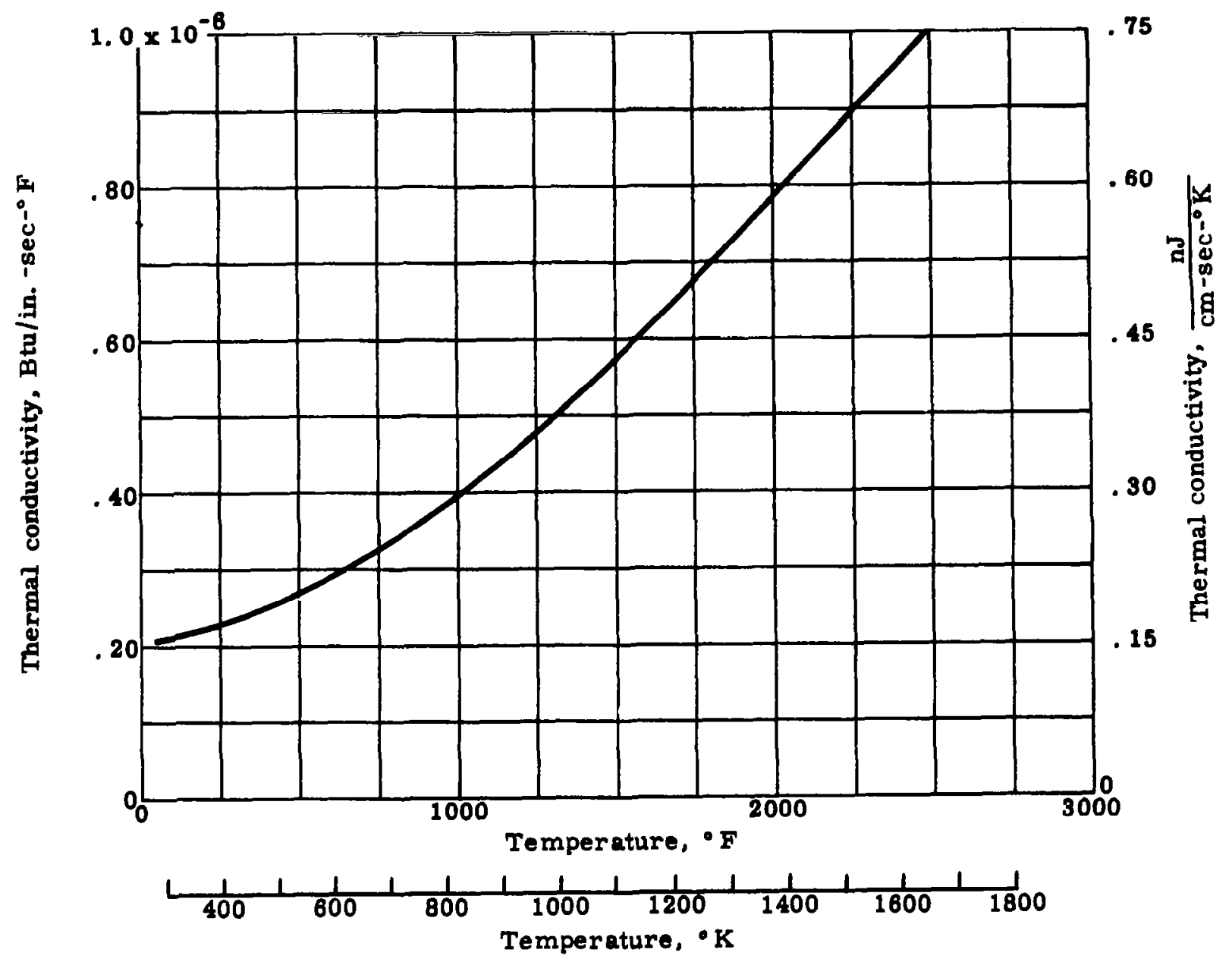

FIGURE 12. MULTILAYER MICROQUARTZ THERMAL CONDUCTIVITY VERSUS TEMPERATURE AT $8 \mathrm{~mm} \mathrm{Hg}$ (Ref. 4) 


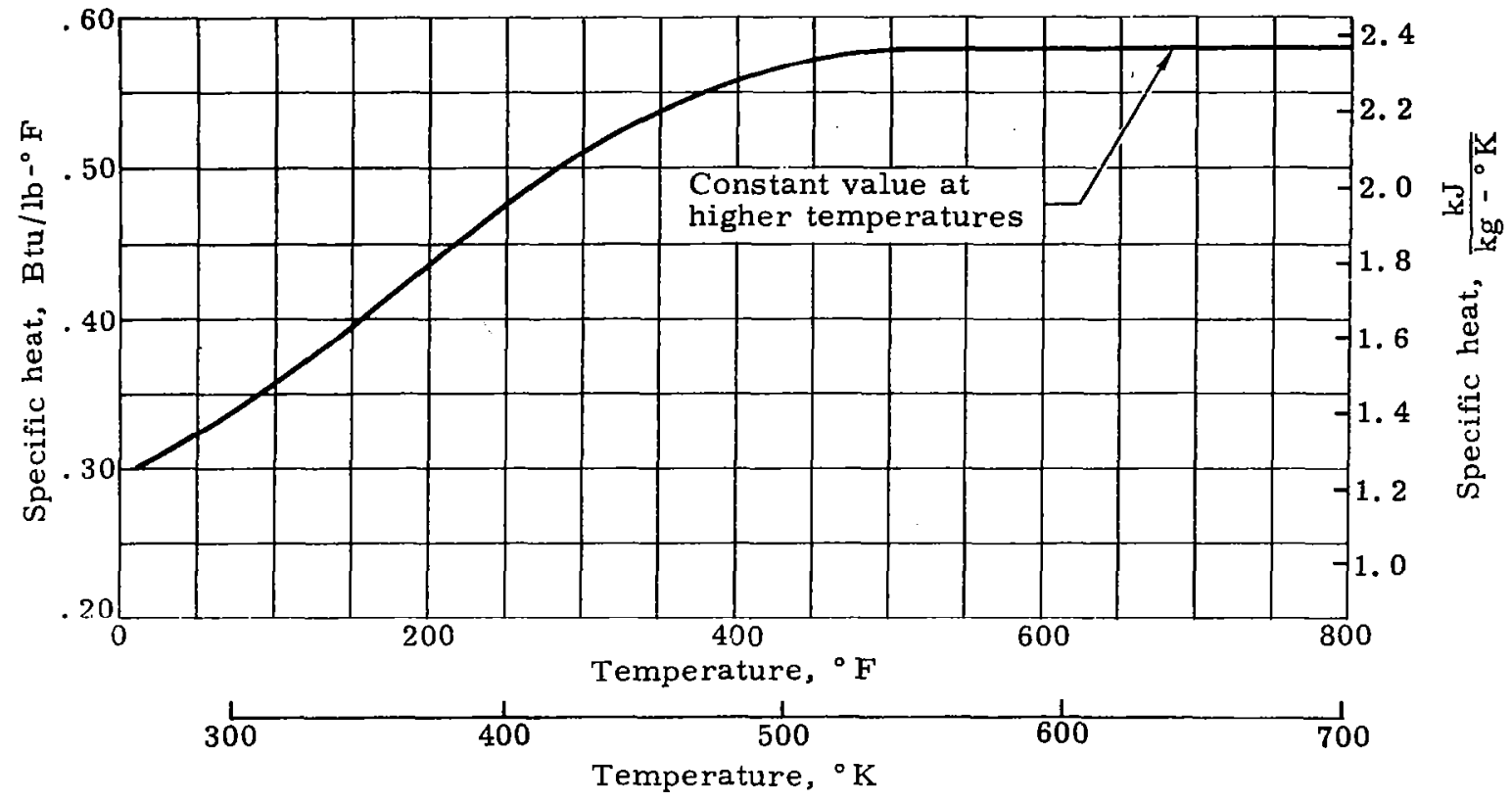

FIGURE 13. LOW DENSITY NYLON PHENOLIC SPECIFIC HEAT FOR VIRGIN AND CHAR MATERIAL

(Ref. 13)

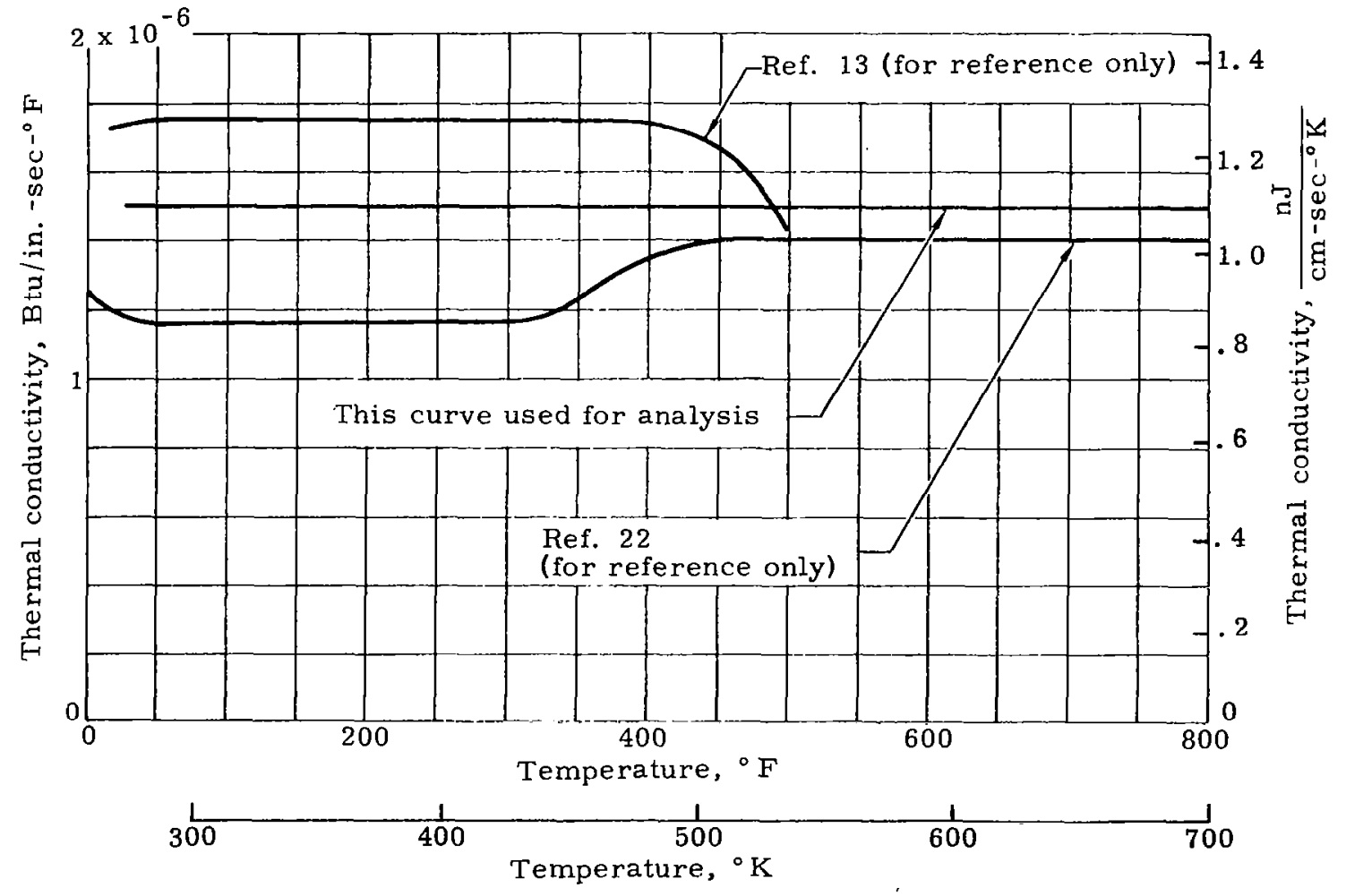

FIGURE 14. LOW DENSITY NYLON PHENOLIC THERMAL CONDUCTIVITY FOR VIRGIN MATERIAL 


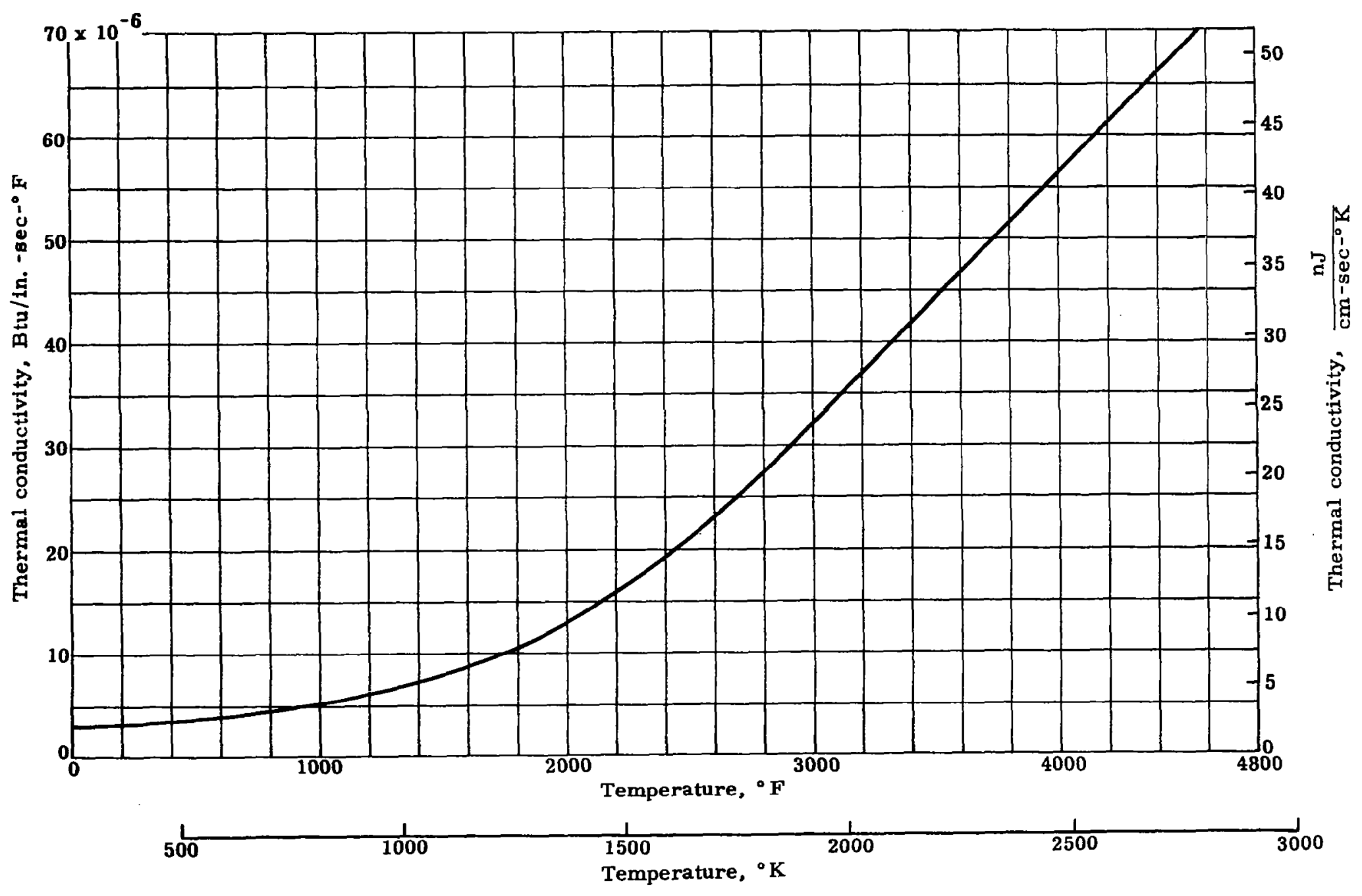

FIGURE 15. LOW DENSITY NYLON PHENOLIC CHAR THERMAL CONDUCTIVITY (Ref. 22) 
客

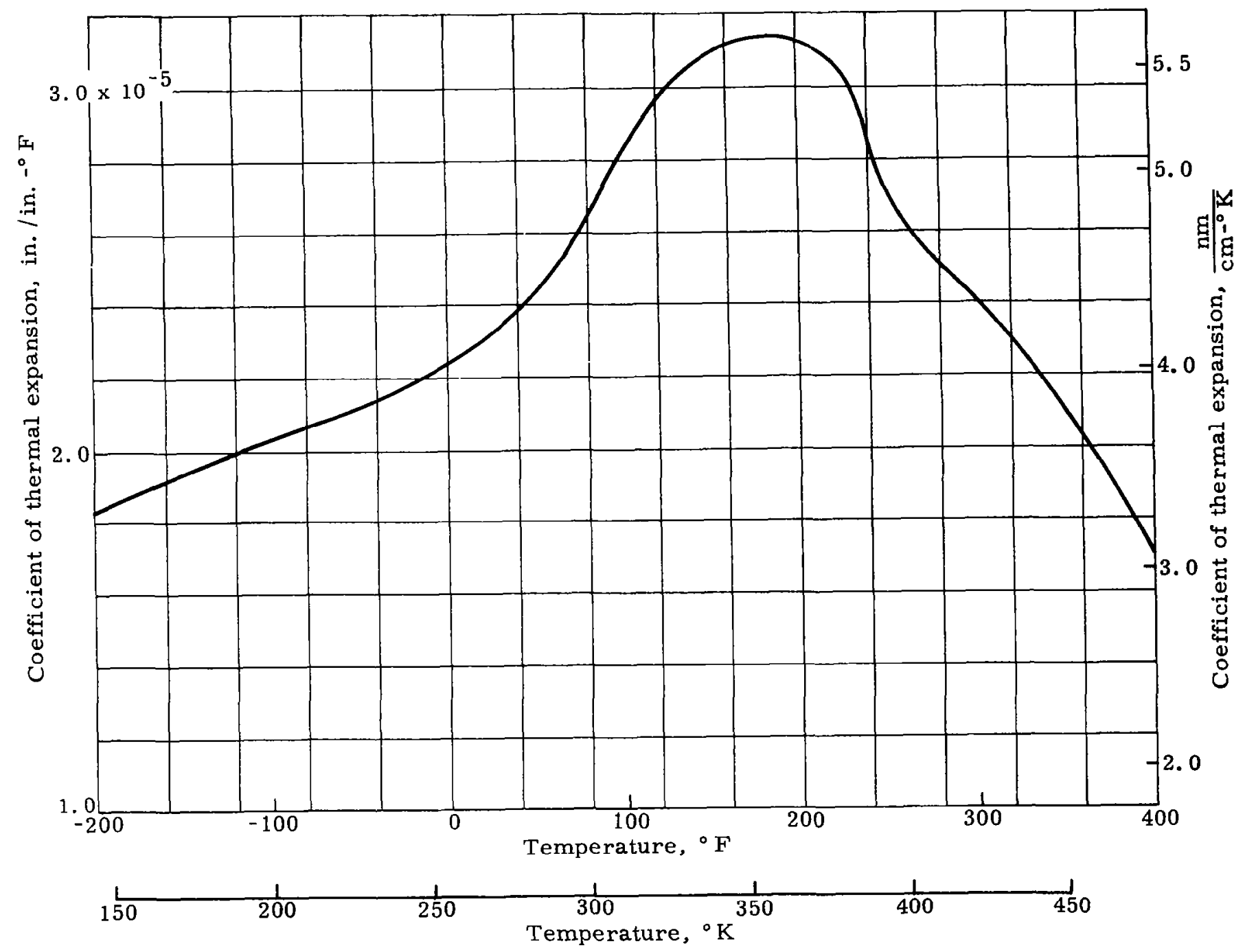

FIGURE 16. INSTANTANEOUS COEFFICIENT OF THERMAL EXPANSION FOR LOW DENSITY NYLON PHENOLIC (Ref. 13) 


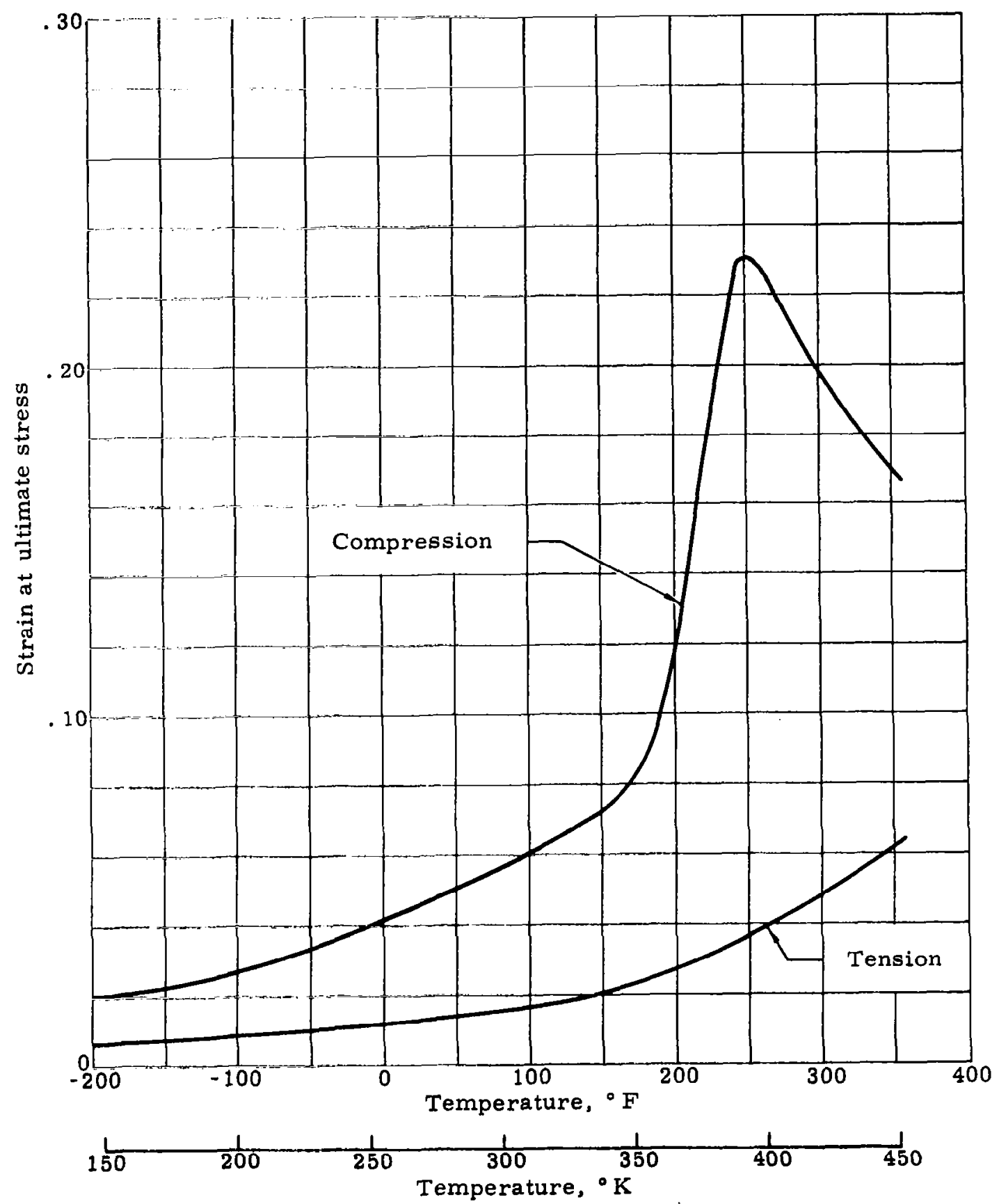

FIGURE 17. LOW DENSITY NYLON PHENOLIC ULTIMATE StRAIN DATA (Ref. 13) 


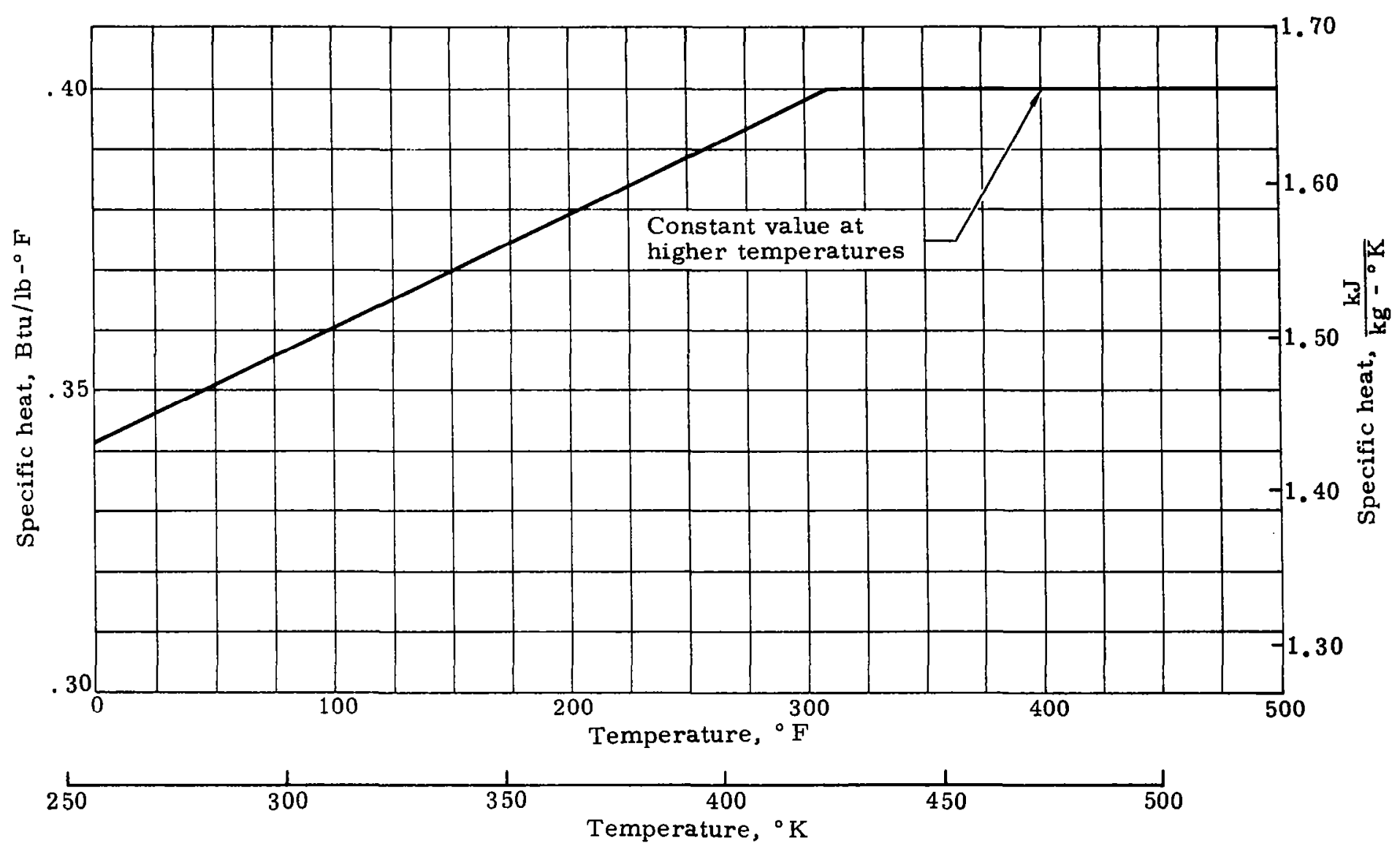

FIGURE 18. NASA 602 SPECIFIC HEAT FOR VIRGIN AND CHAR MATERIAL (Ref. 13) 


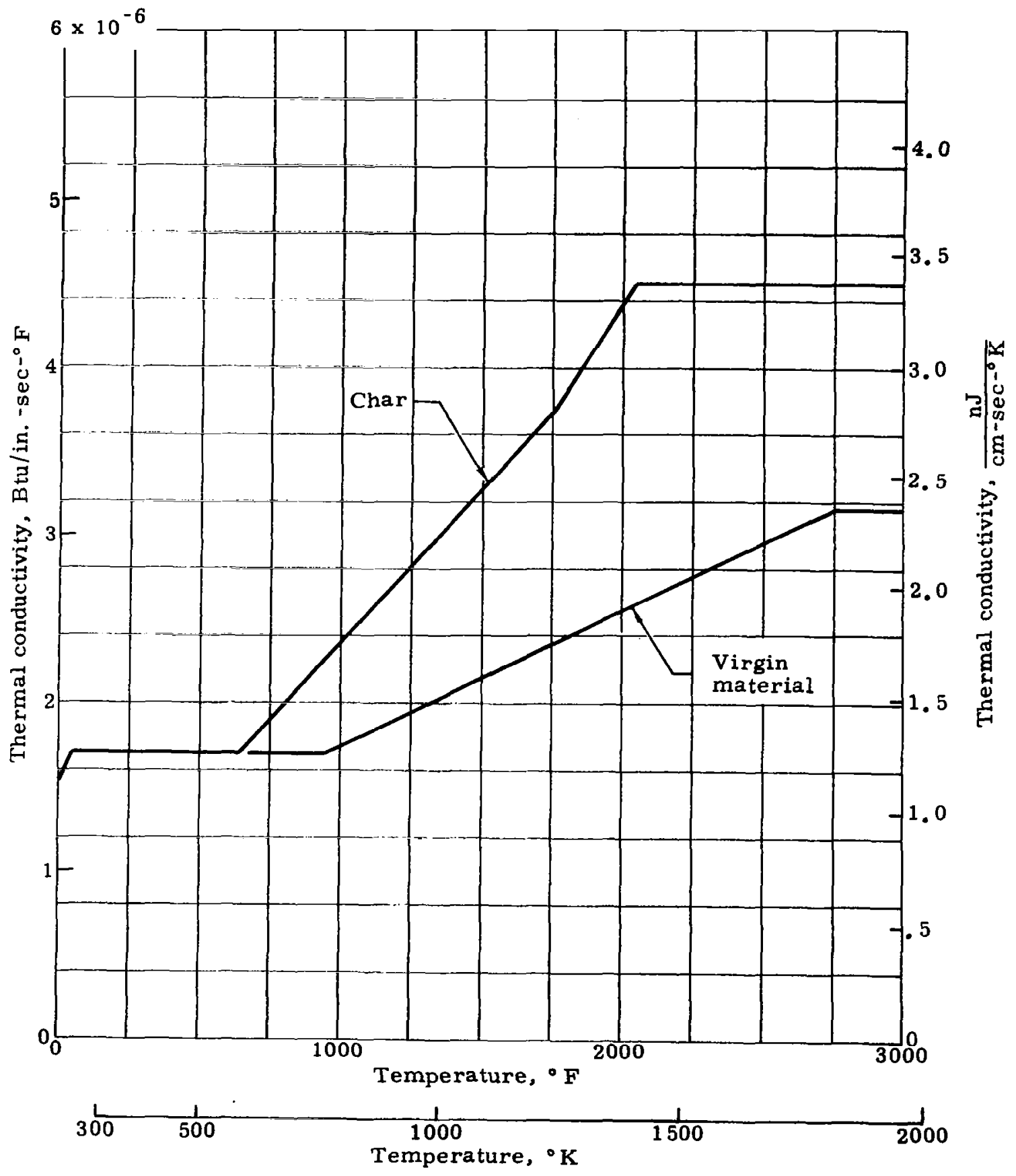

FIGURE 19. NASA 602 THERMAL CONDUCTIVITY 
5

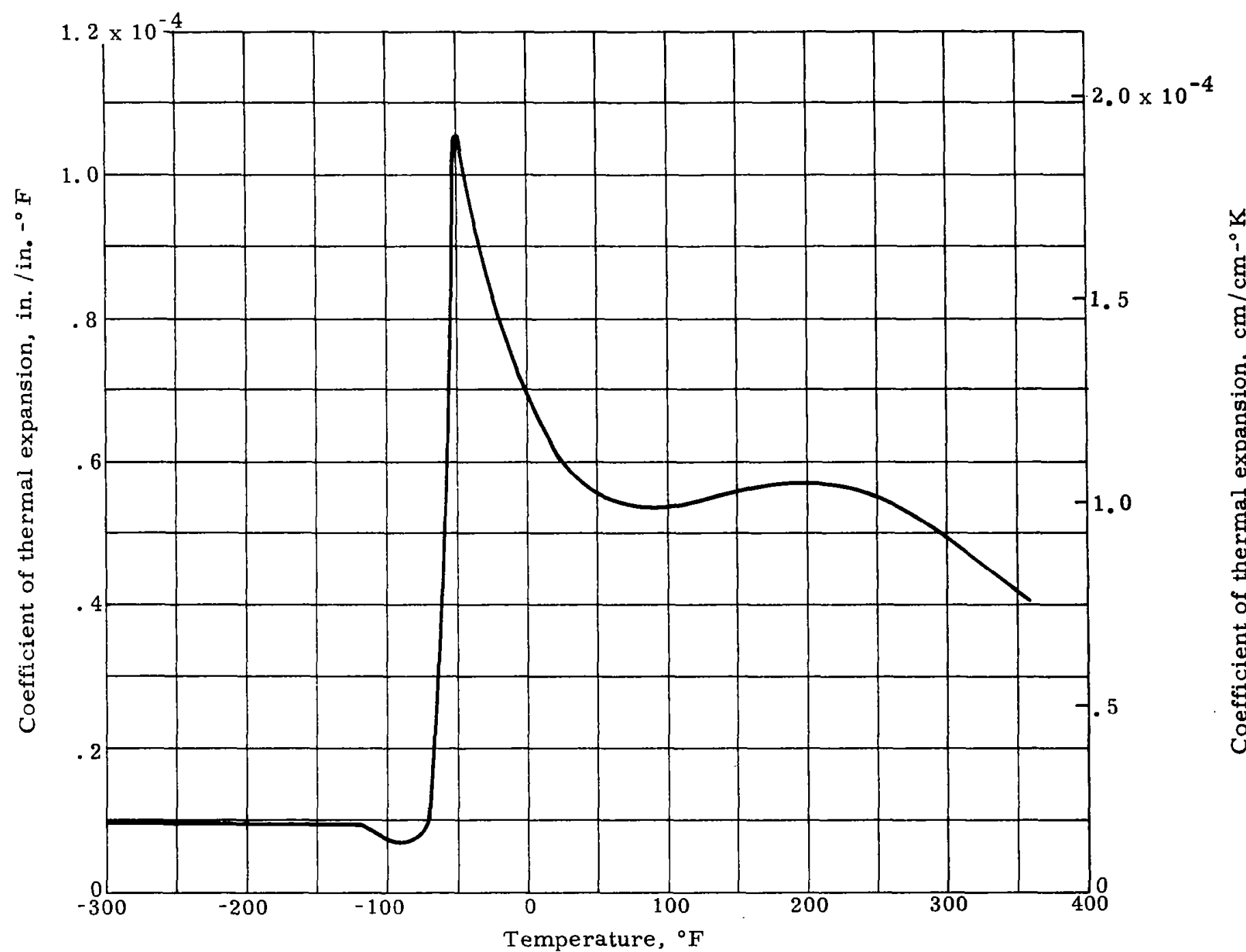

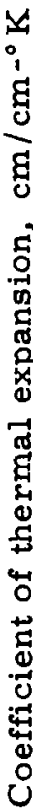

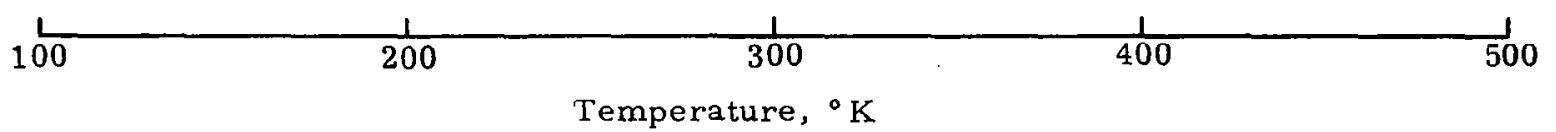

FIGURE 20. NASA 602 INSTANTANEOUS COEFFICIENT OF THERMAL EXPANSION (REF. 19) 

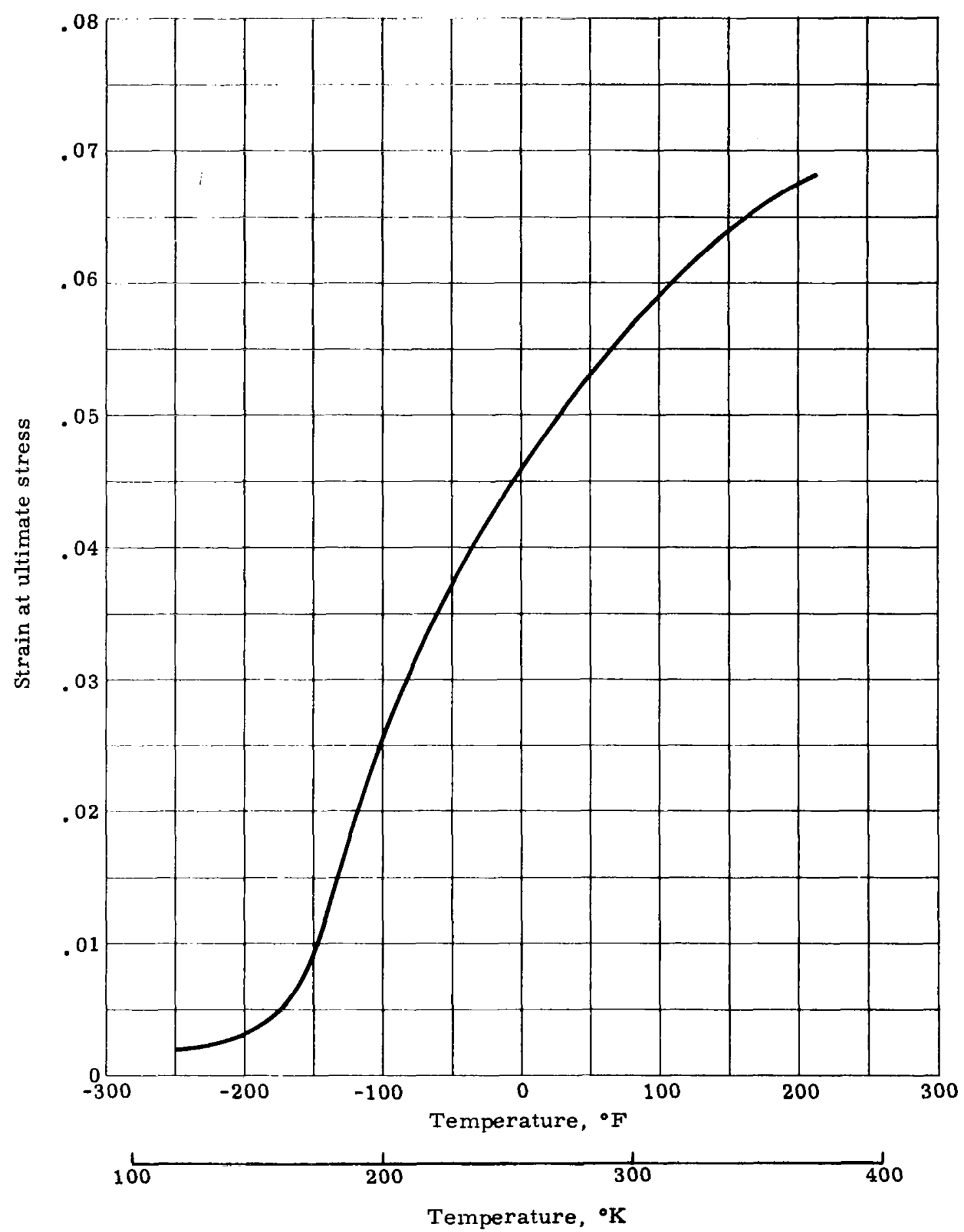

FIGURE 21. NASA 602 IN HONEYCOMB MATRIX ULTIMATE TENSILE STRAIN VERSUS TEMPERATURE (REF. 13) 
$\stackrel{\mathbb{N}}{\mathbb{N}}$

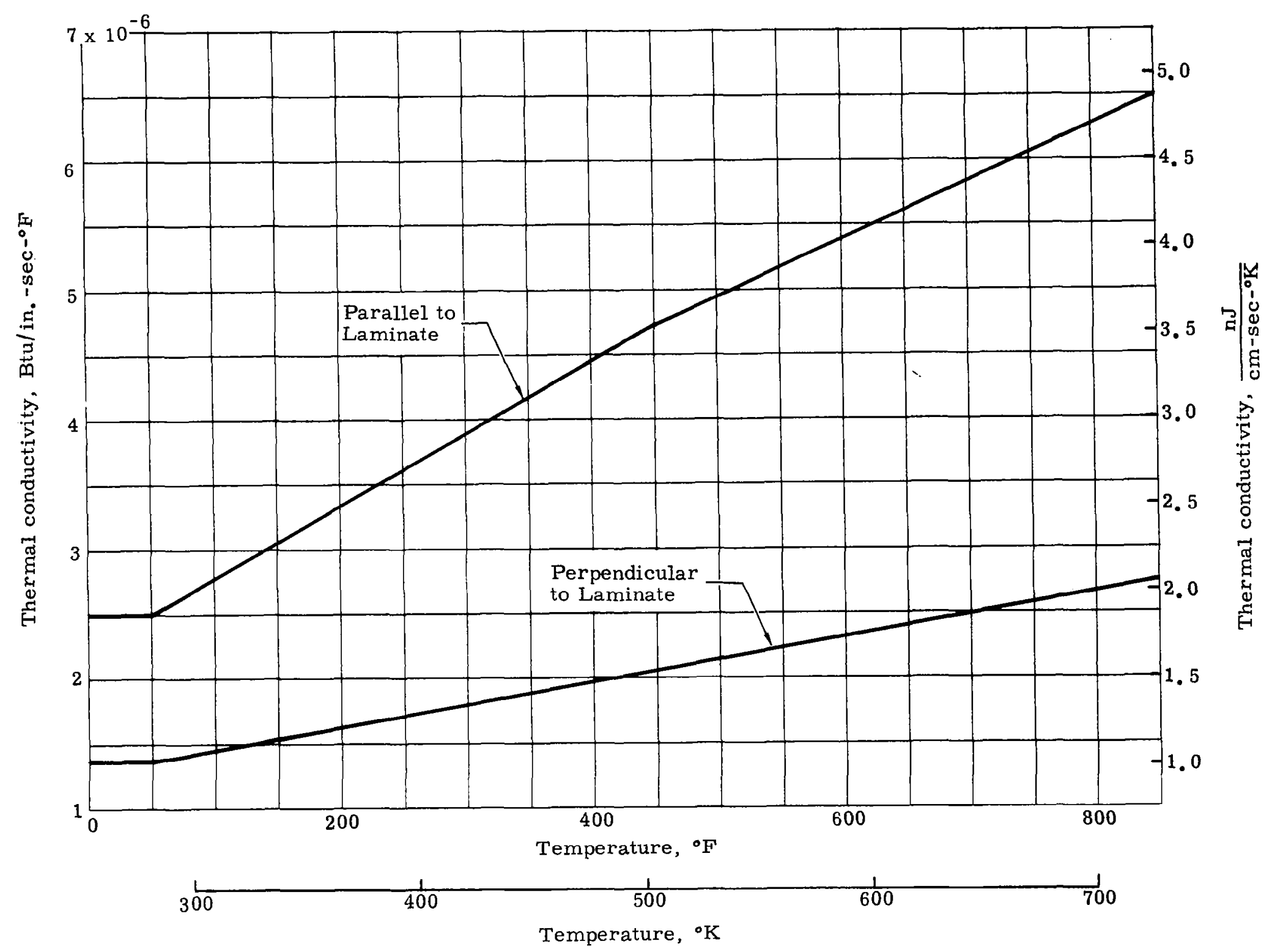

FIGURE 22. PHENOLIC/GLASS LAMINATE THERMAL CONDUCTIVITY 


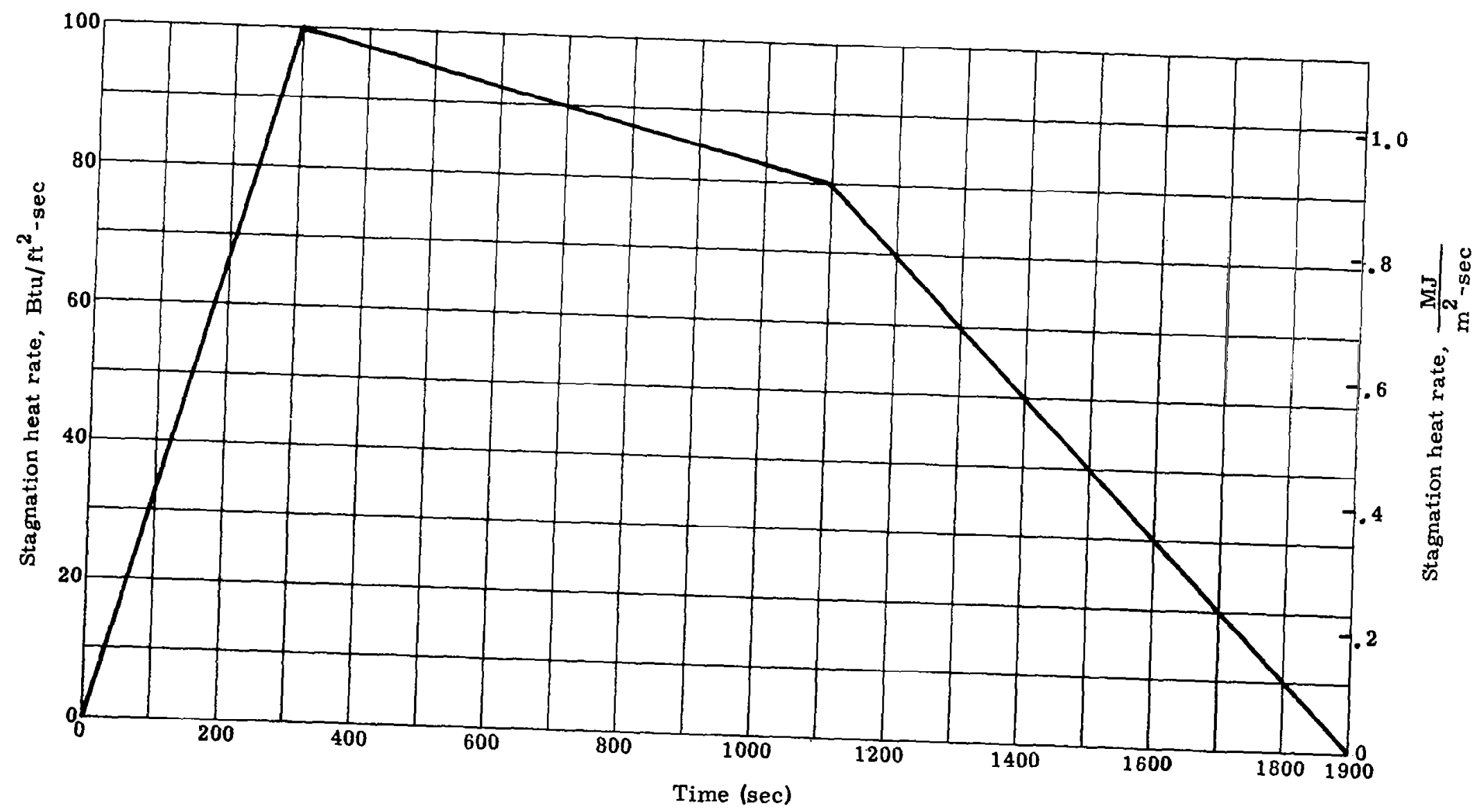

FIGURE 23. IDEALIZED RE-ENTRY HEATING PULSE 
$\stackrel{\leftrightarrow}{\stackrel{\leftrightarrow}{\leftrightarrow}}$

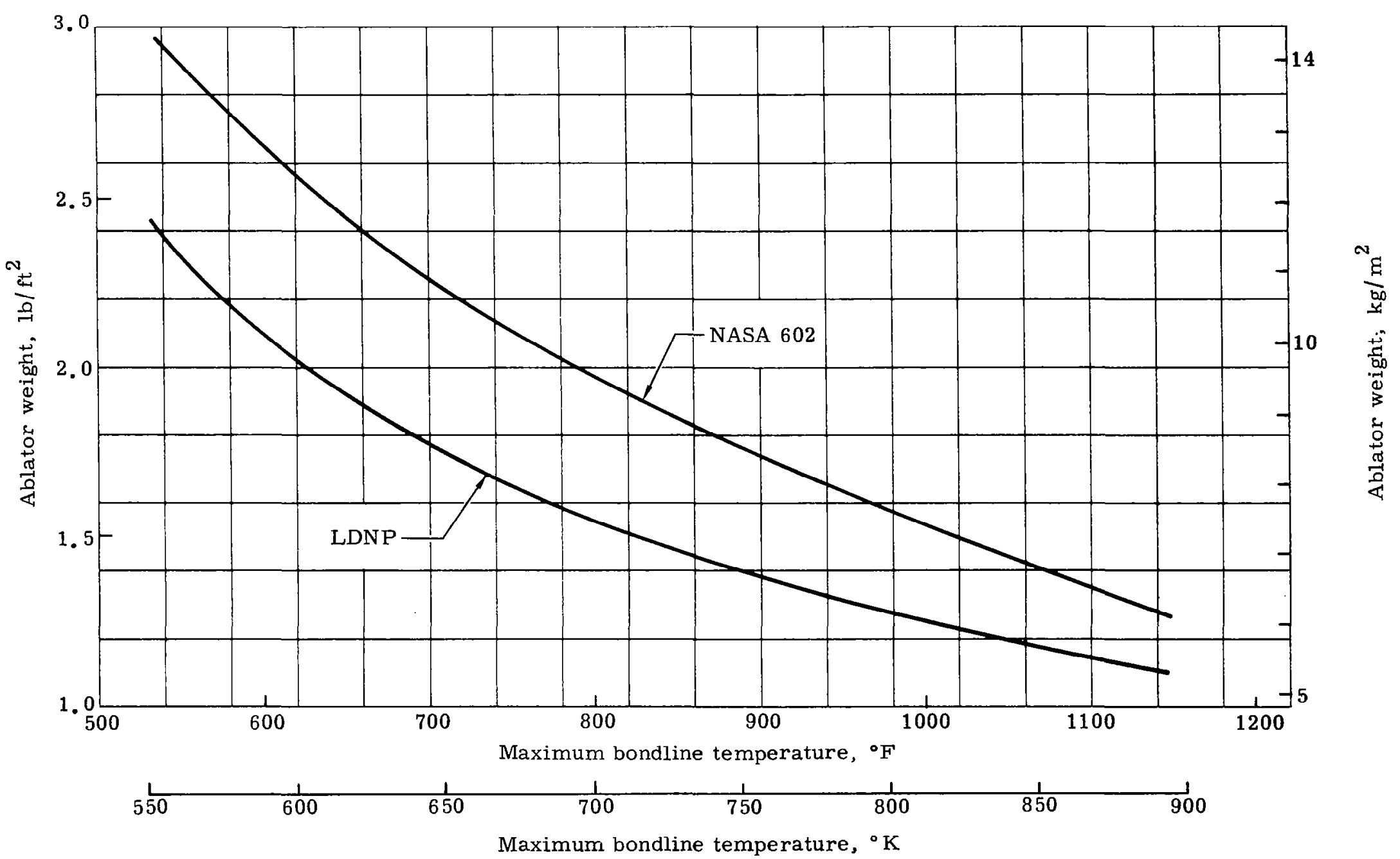

FIGURE 24. ABLATOR WEIGHT VERSUS MAXIMUM BONDLINE TEMPERATURE 


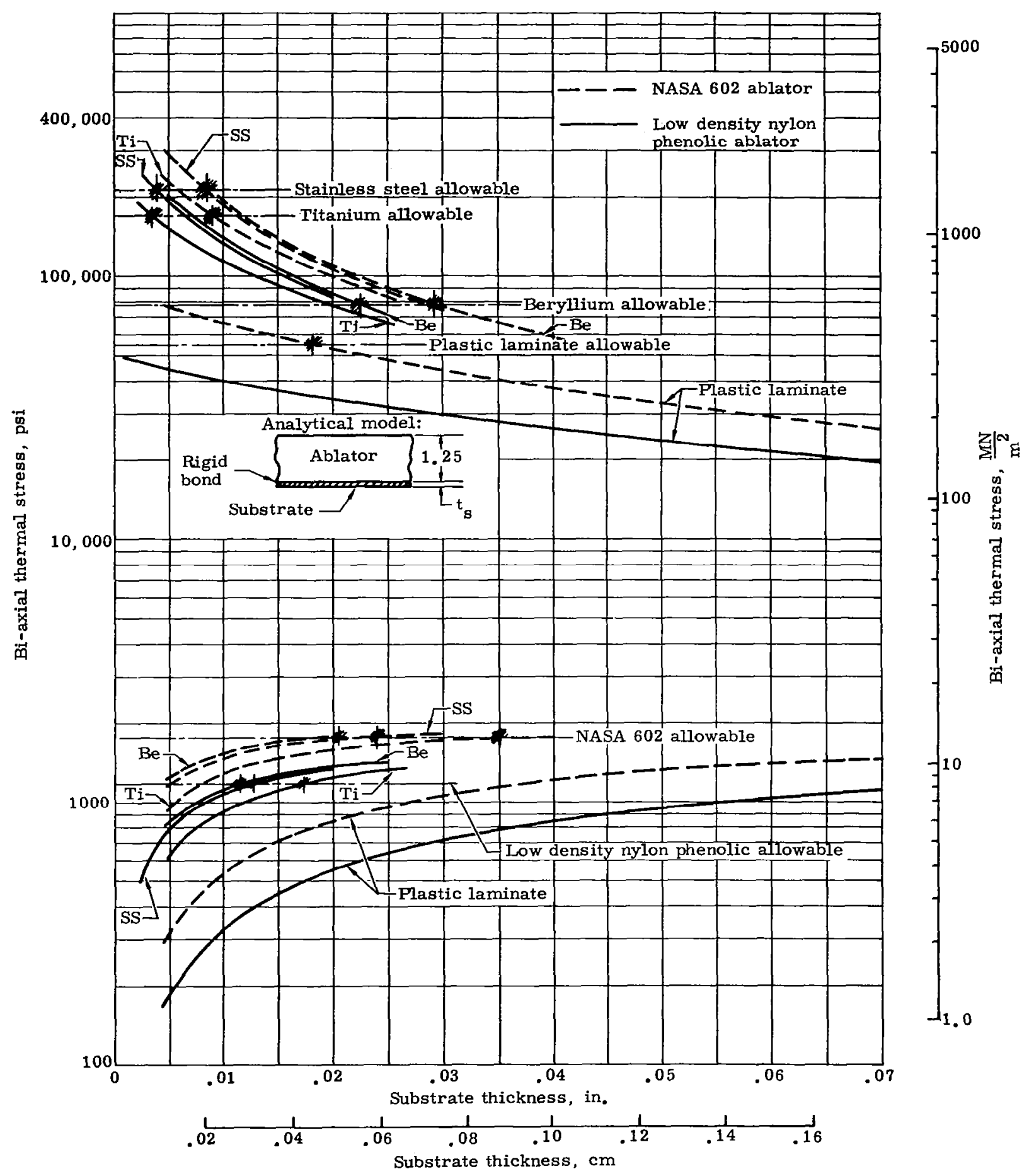

FIGURE 25. THERMAL STRESS COMPARISONS (UNIFORM COLD SOAK AT $-110^{\circ} \mathrm{F}$ OR $194.4^{\circ} \mathrm{K}$ ) 

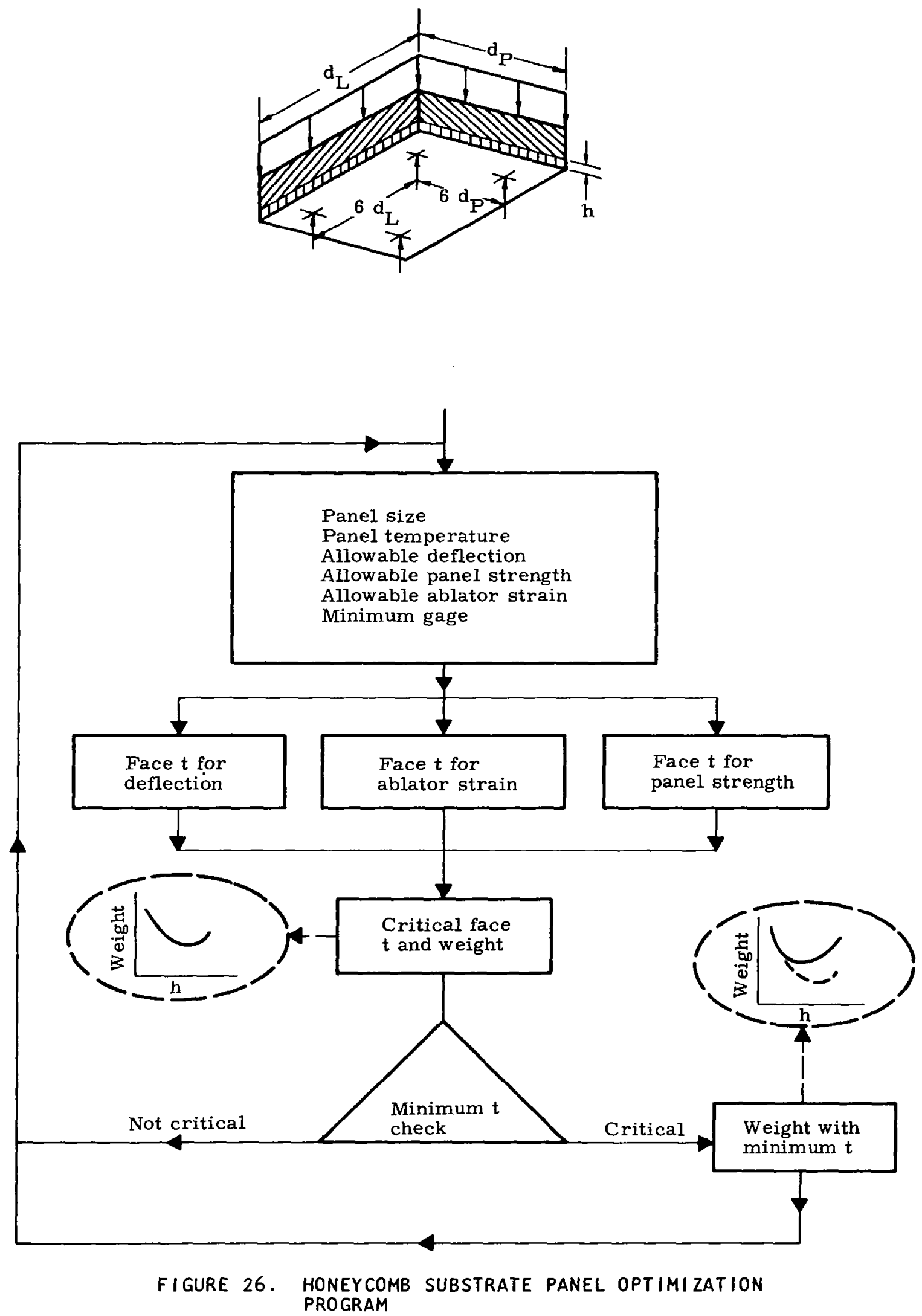


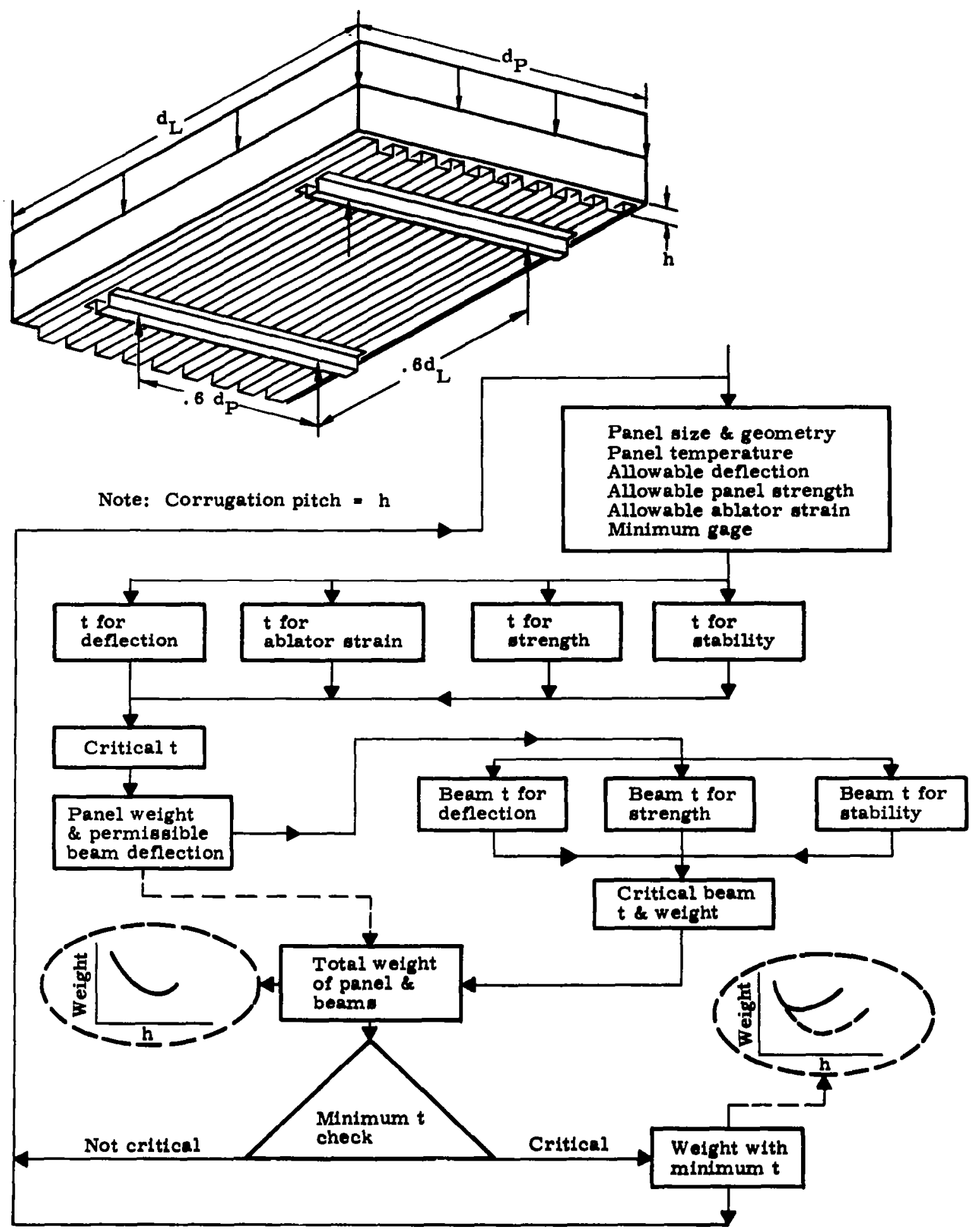

FIGURE 27. SINGLE SKIN CORRUGATION SUBSTRATE PANEL OPTIMIZATION PROGRAM 
$\underset{\infty}{\rightleftarrows}$

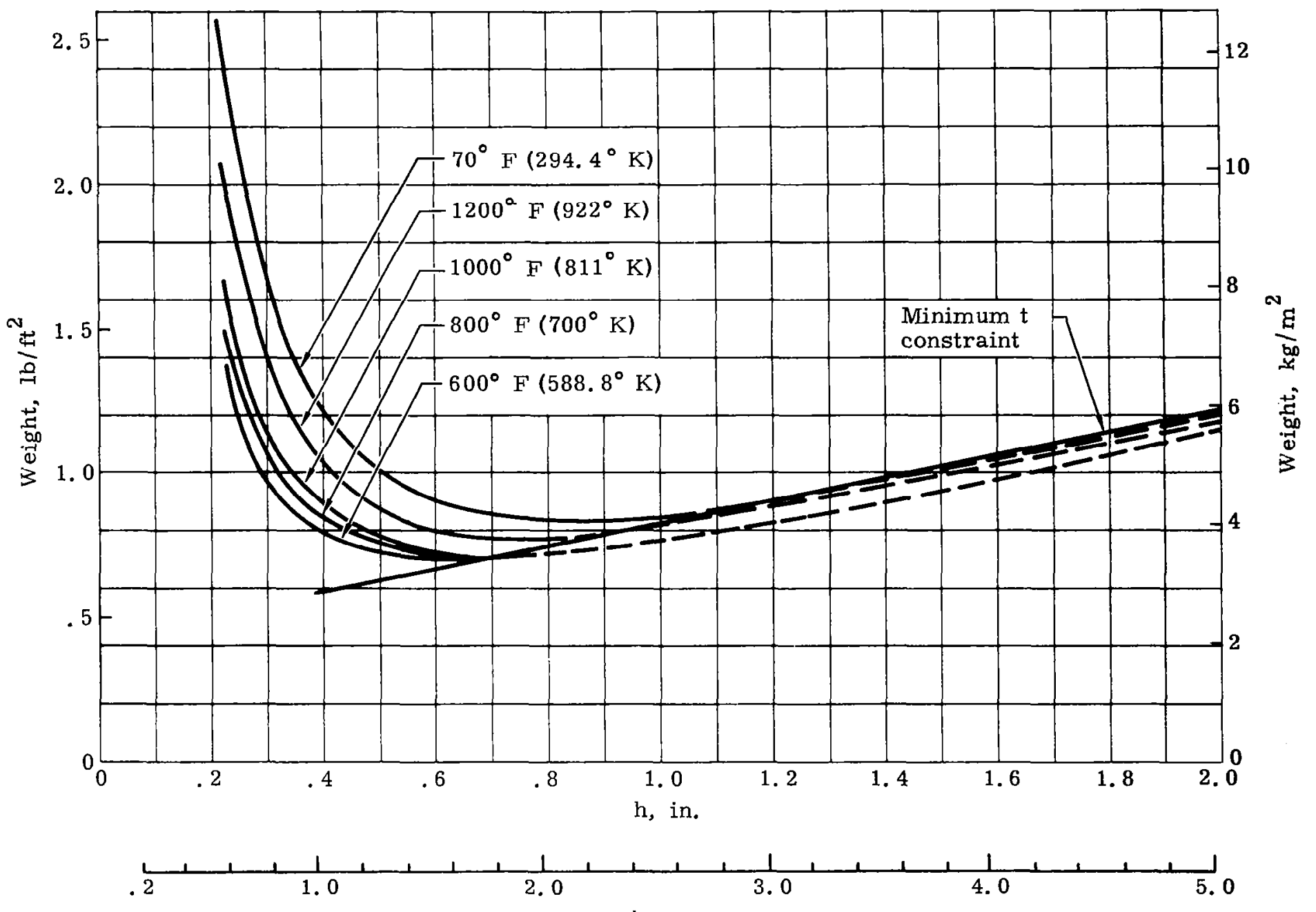

h, $\mathbf{c m}$

FIGURE 28. STAINLESS STEEL HONEYCOMB PANEL OPTIMUM WEIGHT CURVES 


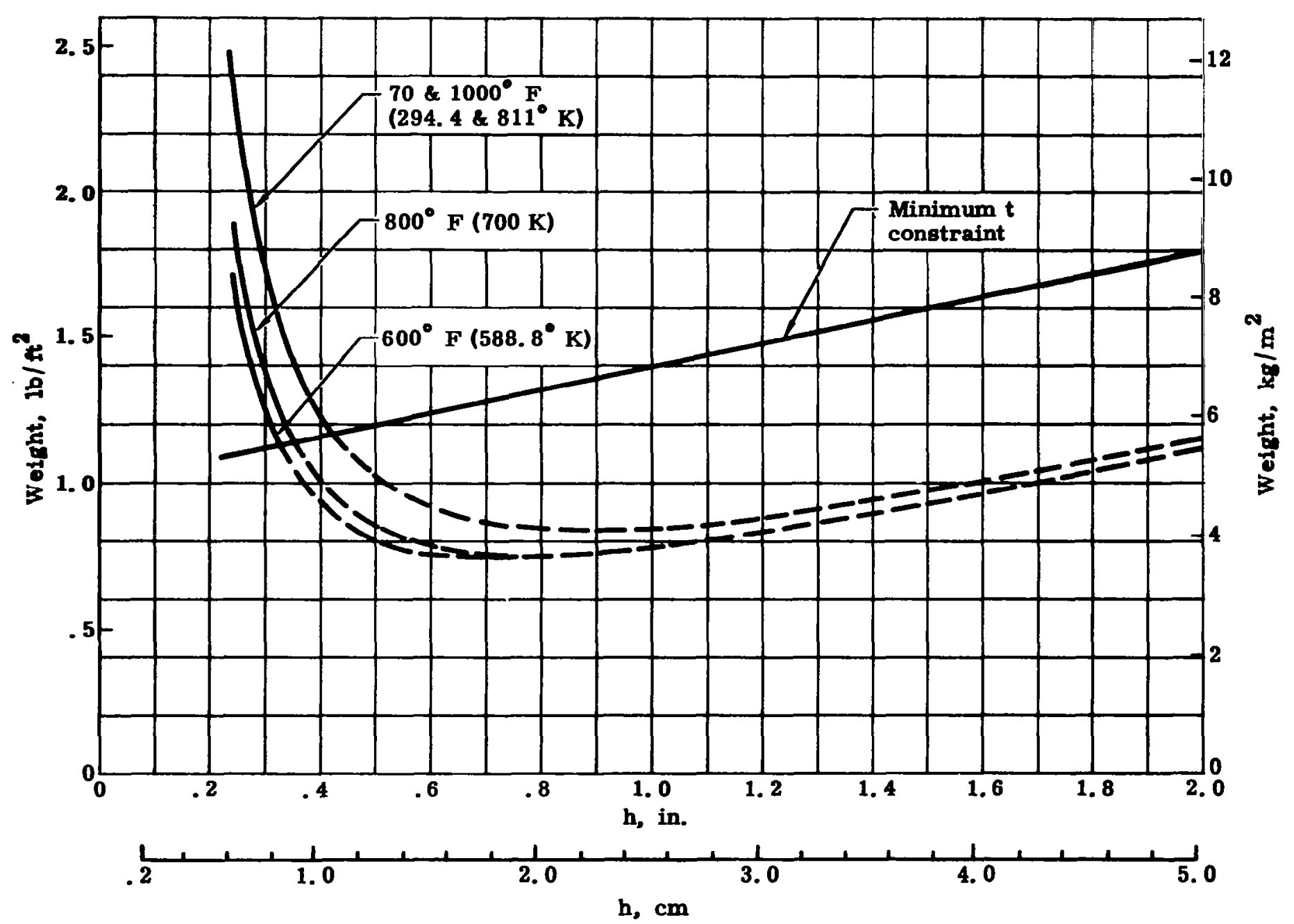

FIGURE 29. TITANIUM HONEYCOMB PANEL OPTIMUM WEIGHT CURVES 


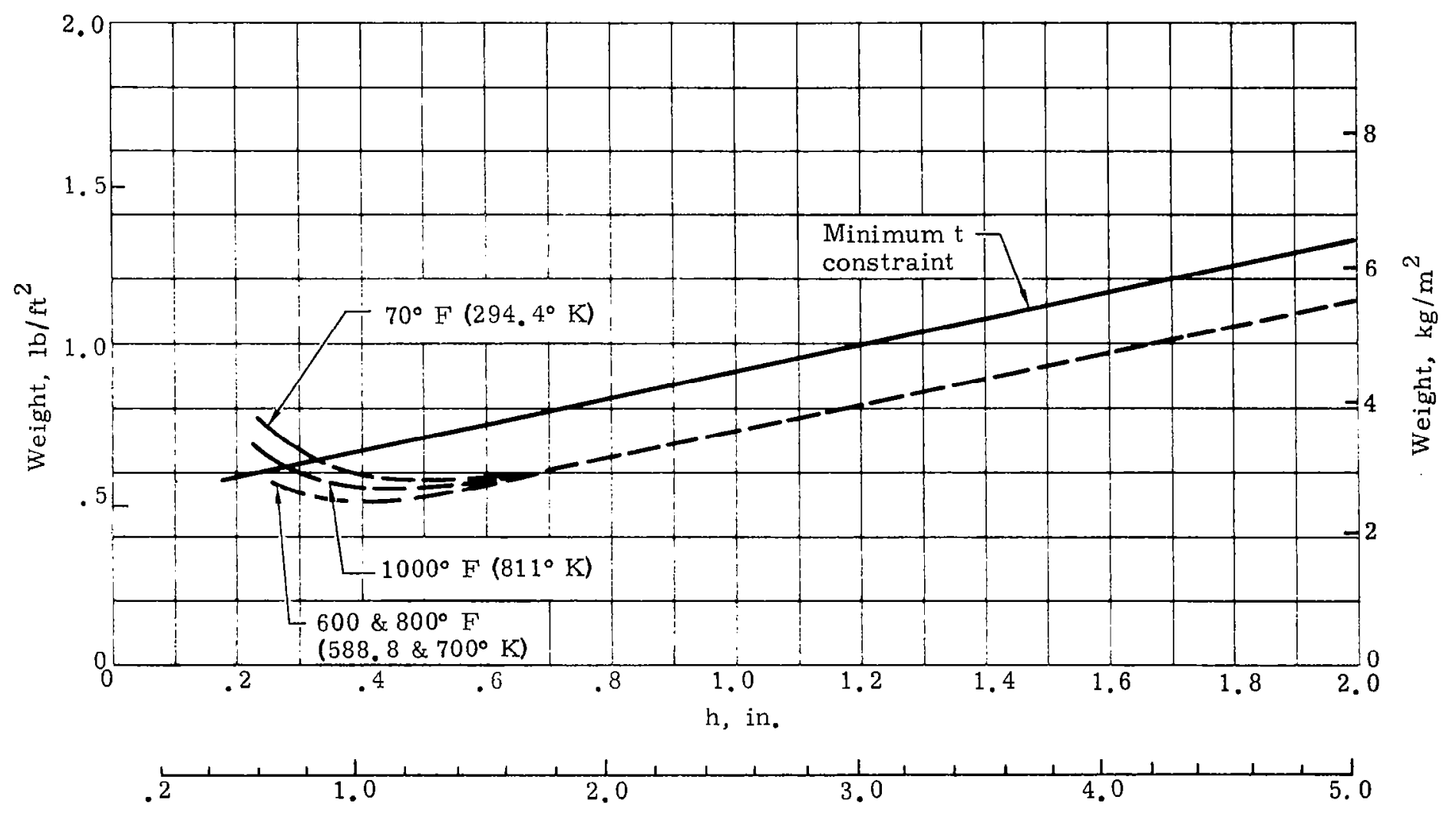

$h, \mathrm{~cm}$

FIGURE 30. BERYLLIUM HONEYCOMB PANEL OPTIMUM WEIGHT CURVES 


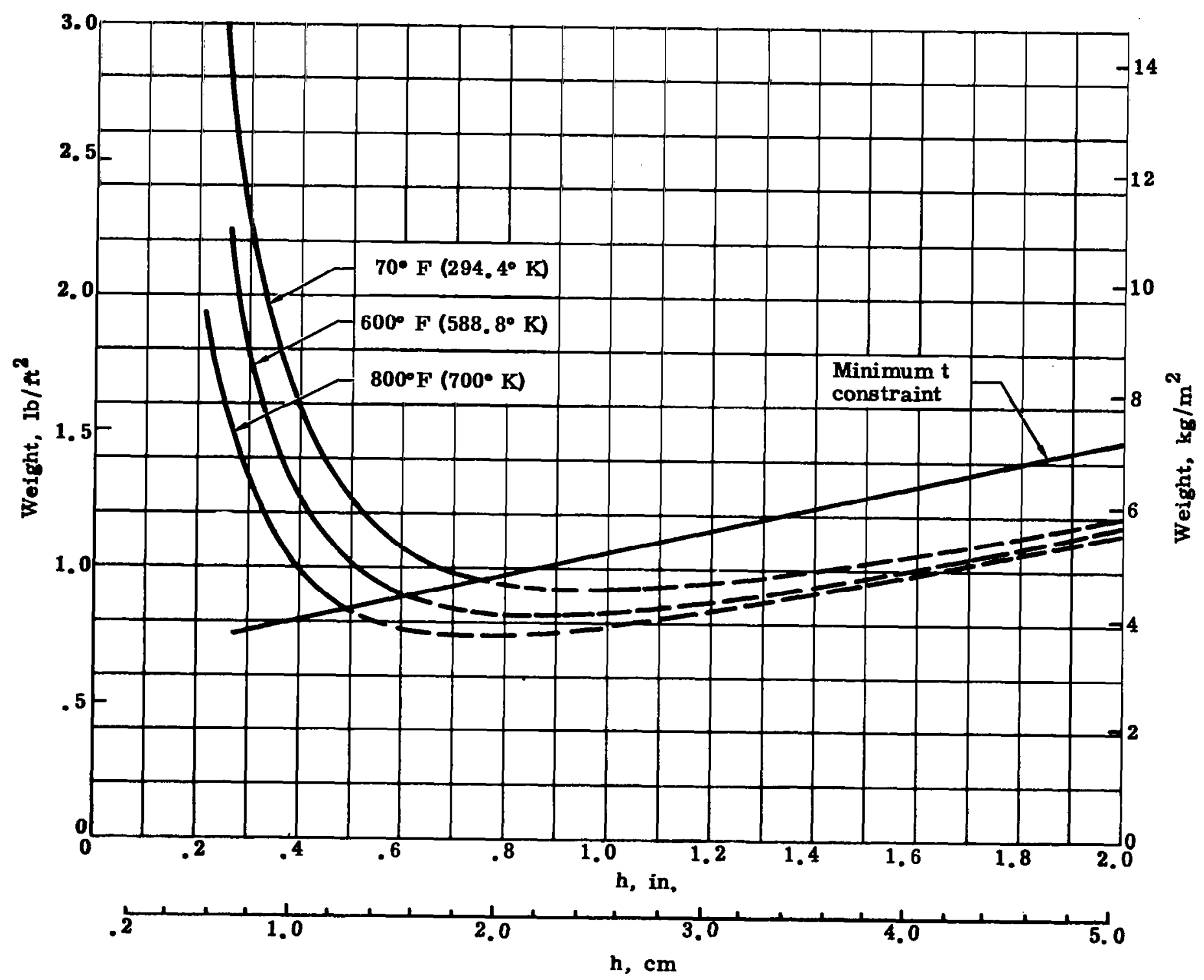




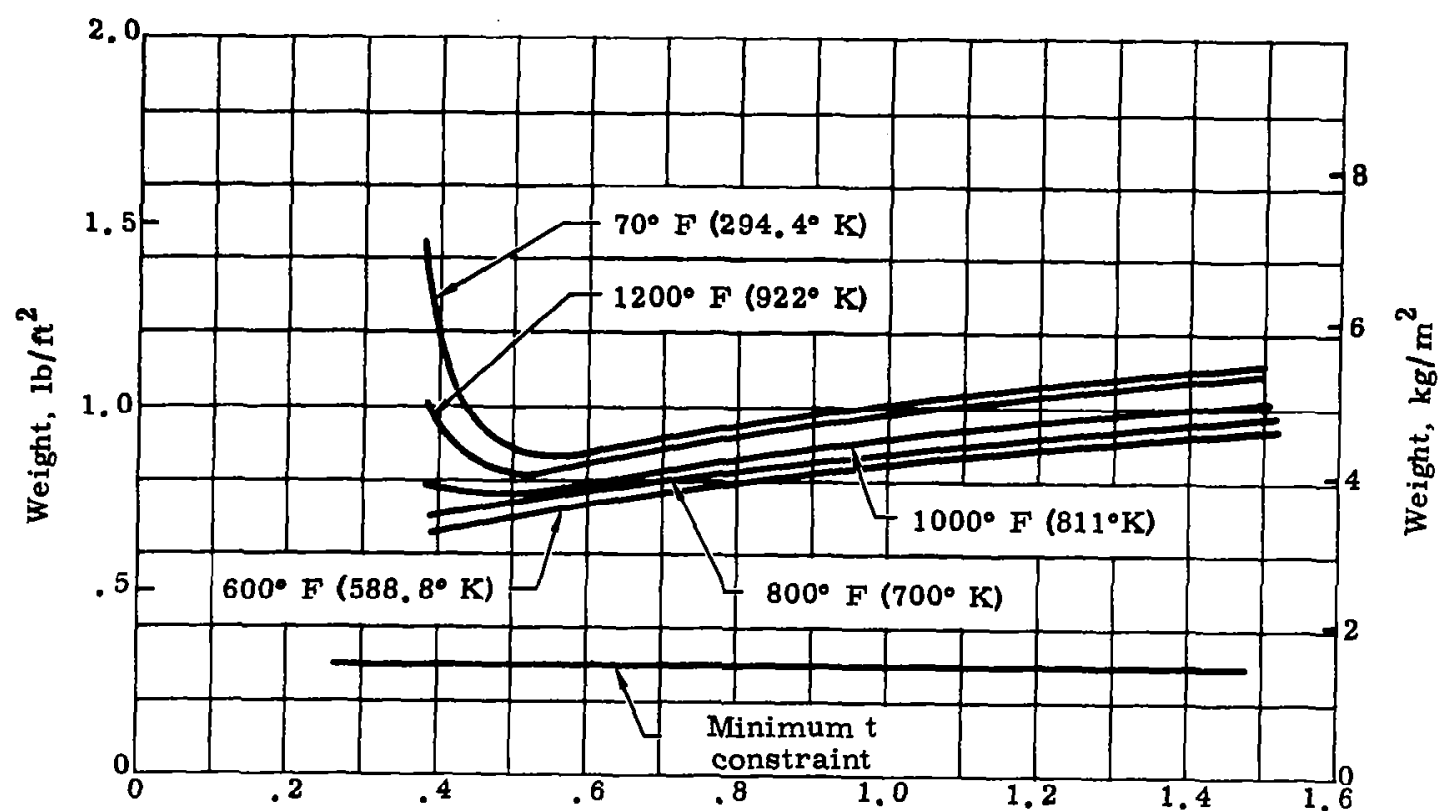

$h$, in.

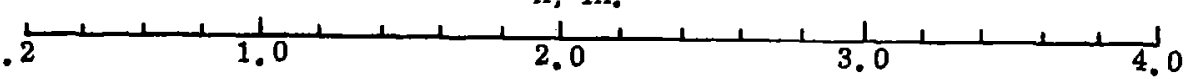

h, $\mathrm{cm}$

FIGURE 32. STAINLESS STEEL SINGLE SKIN CORRUGATION PANEL OPT IMUM WEIGHT CURVES

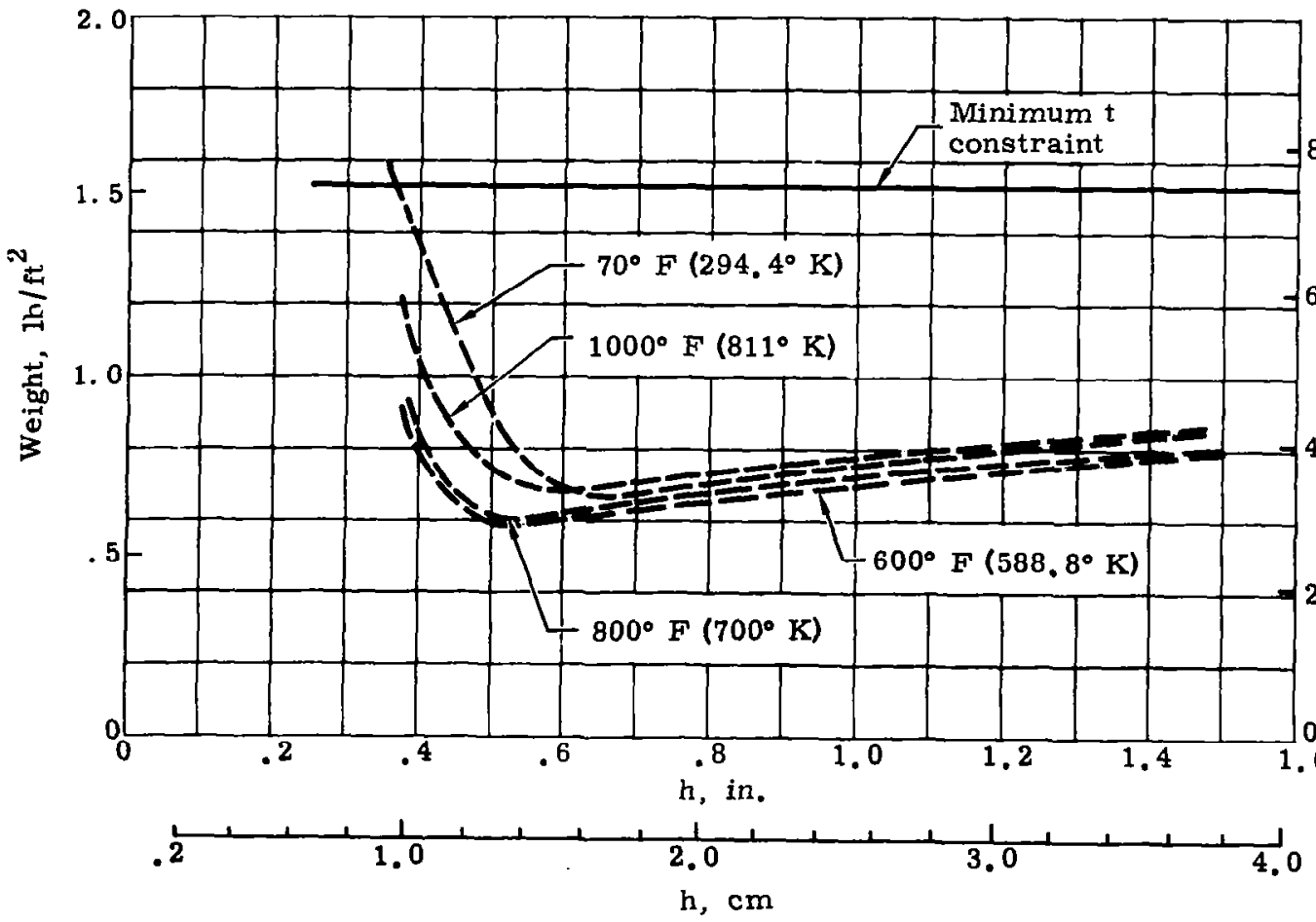

FIGURE 33. TITANIUM SINGLE SKIN CORRUGATION PANEL OPTIMUM WEIGHT
CURVES 


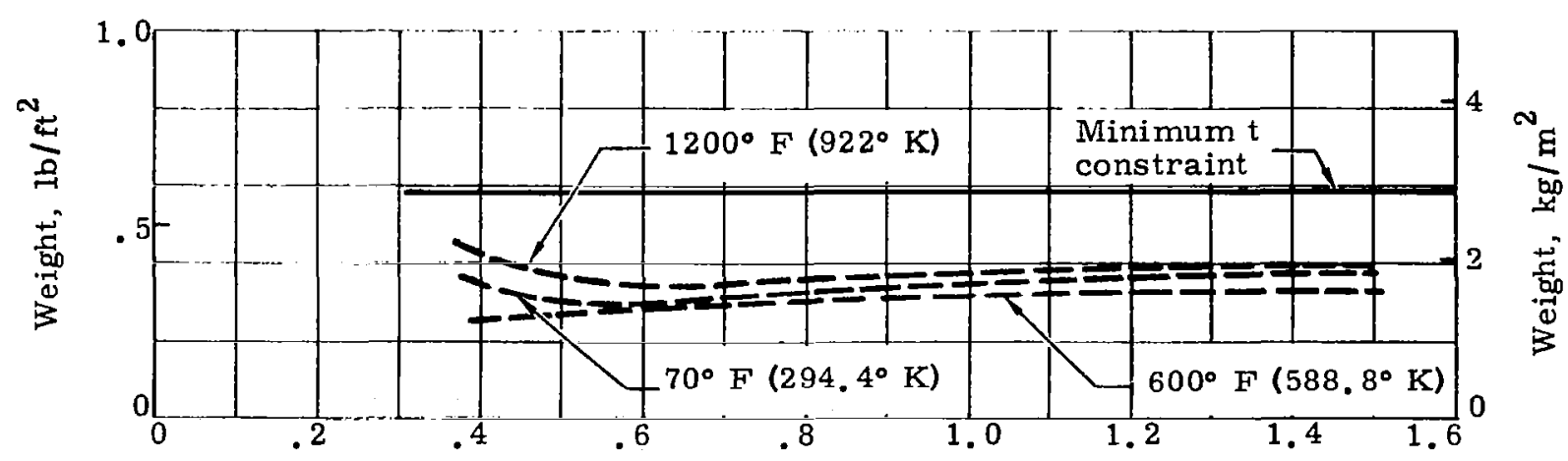

$h$, in.

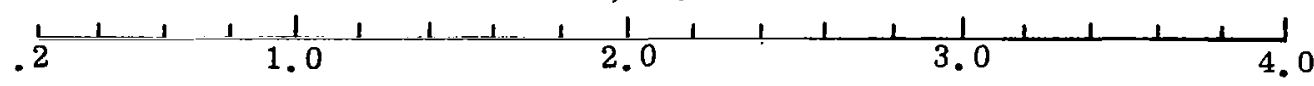

$\mathrm{h}, \mathrm{cm}$

FIGURE 34. BERYLLIUM SINGLE SKIN CORRUGATION PANEL OPTIMUM WEIGHT CURVES

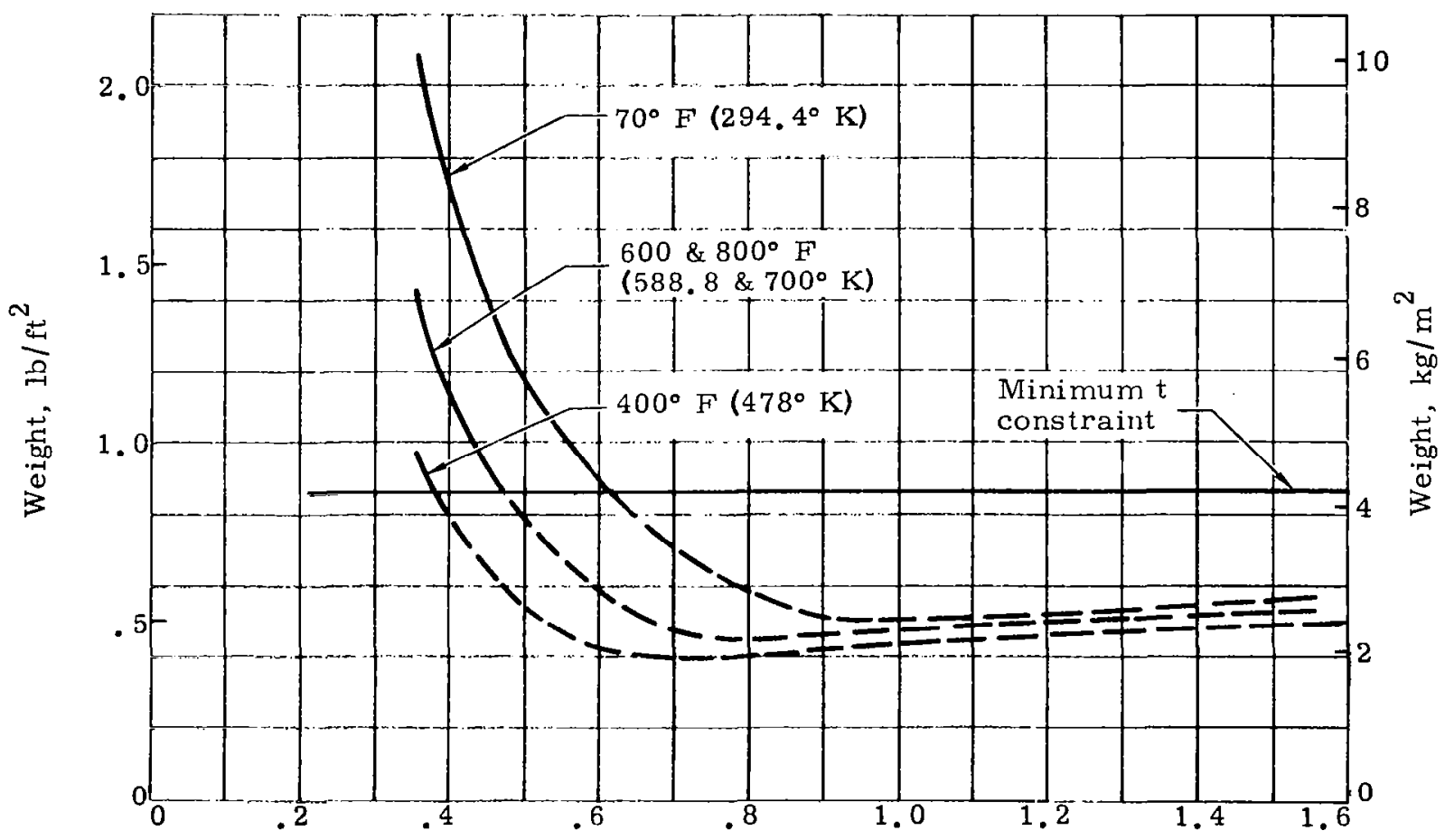

$h$, in.

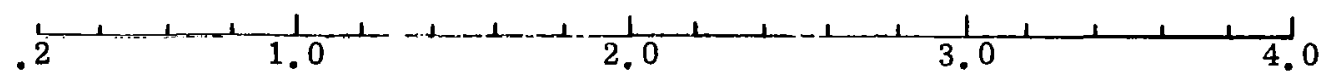

$\mathrm{h}, \mathrm{cm}$

FIGURE 35. PHENOLIC/GLASS LAMINATE SINGLE SKIN CORRUGATION PANEL OPT IMUM WEIGHT CURVES 
$\widetilde{\Sigma}$

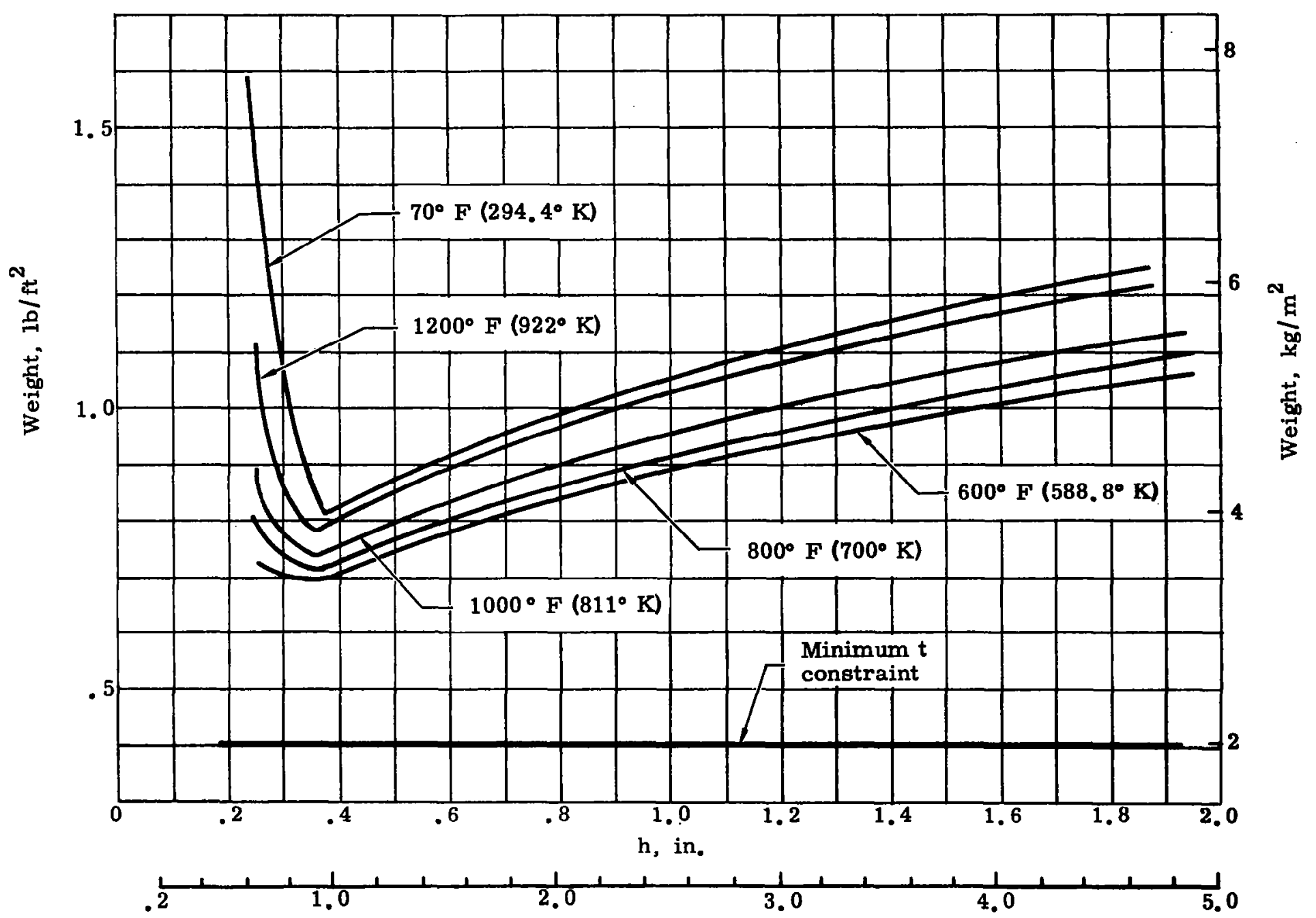

h, cm

FIGURE 36. STAINLESS STEEL DOUBLE SKIN CORRUGATION PANEL OPTIMUM WEIGHT CURVES 


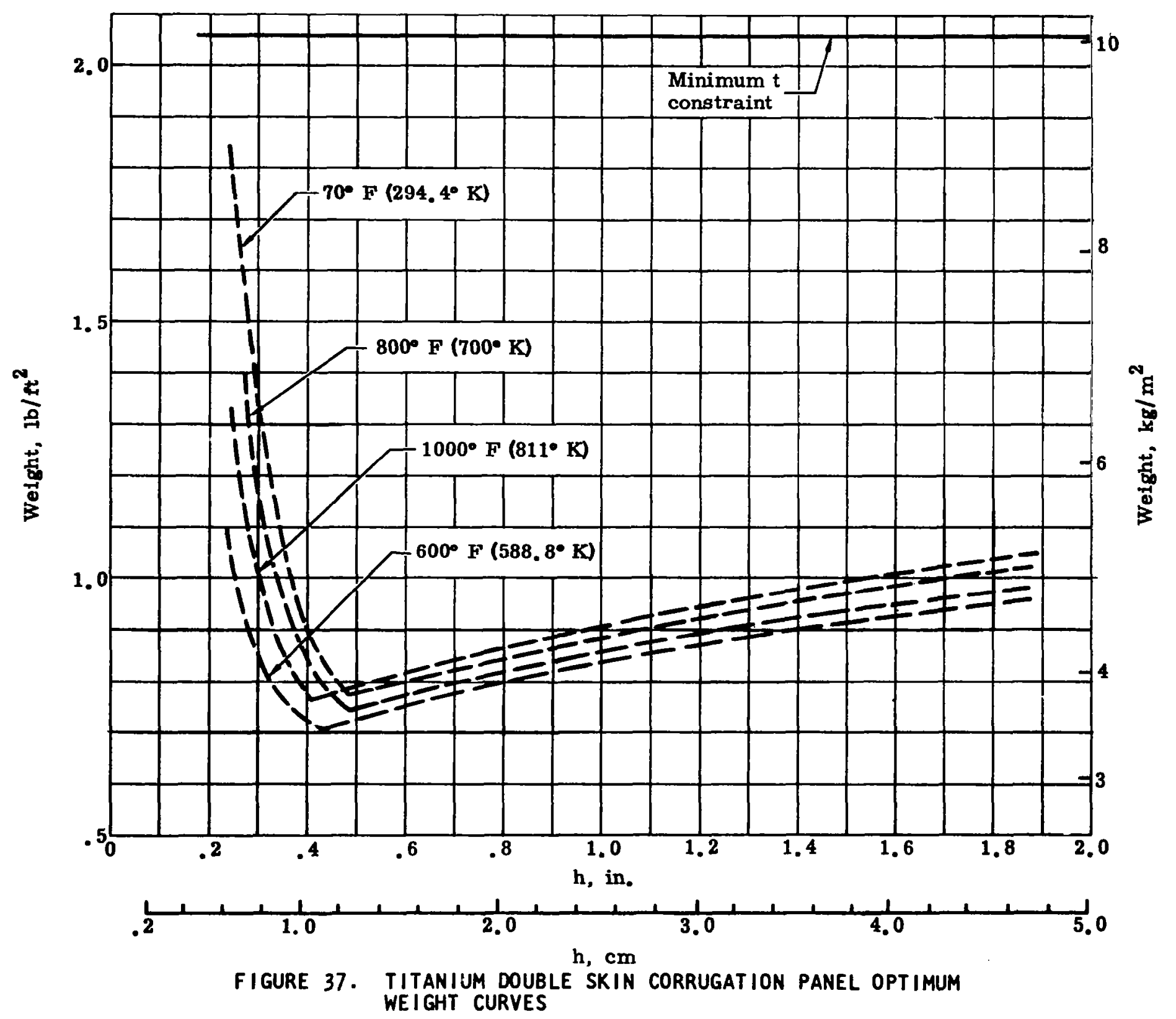


농
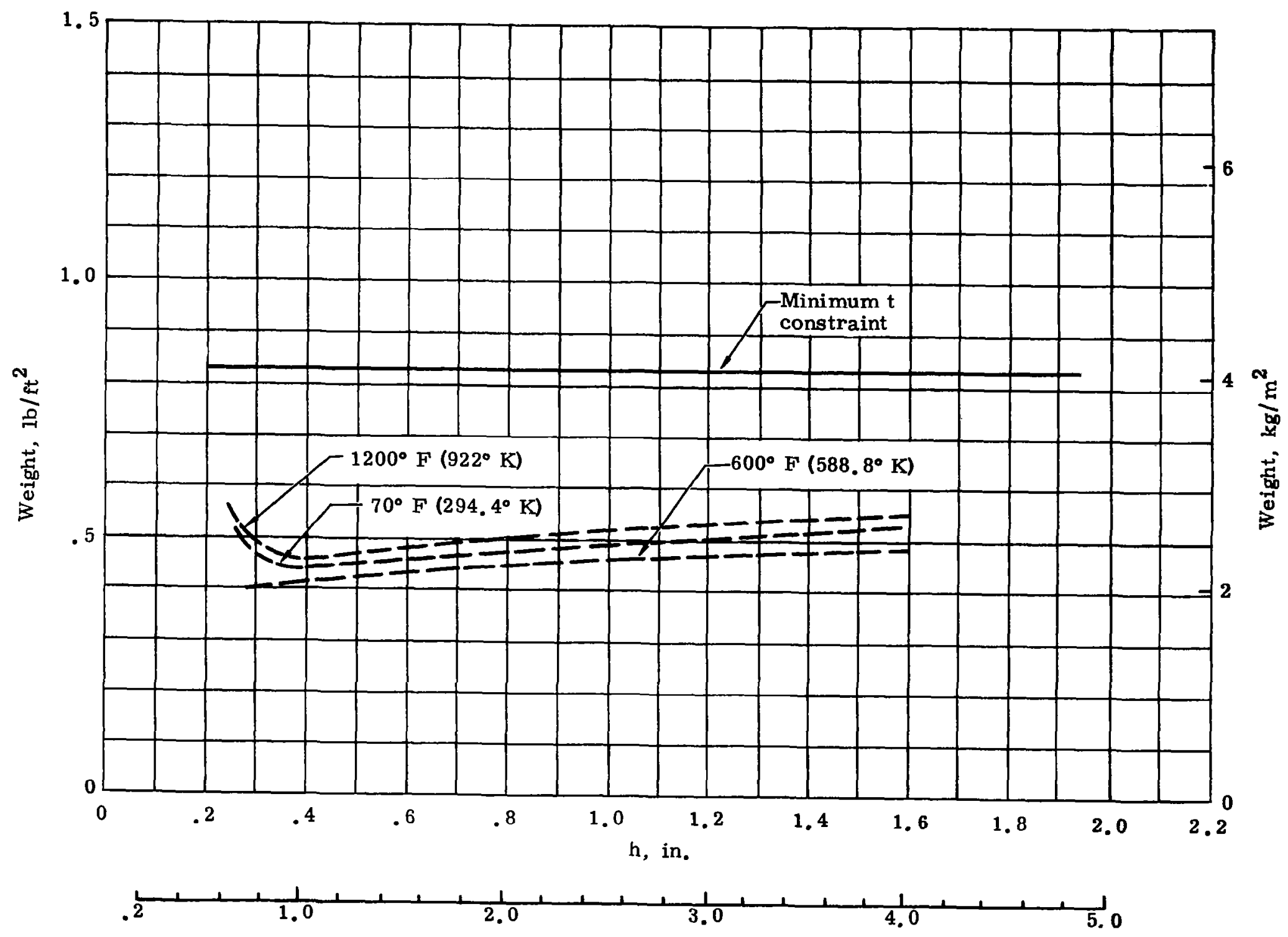

$\mathrm{h}, \mathrm{cm}$

FIGURE 38. BERYLLIUM DOUBLE SKIN CORRUGATION PANEL OPTIMUM WEIGHT CURVES 


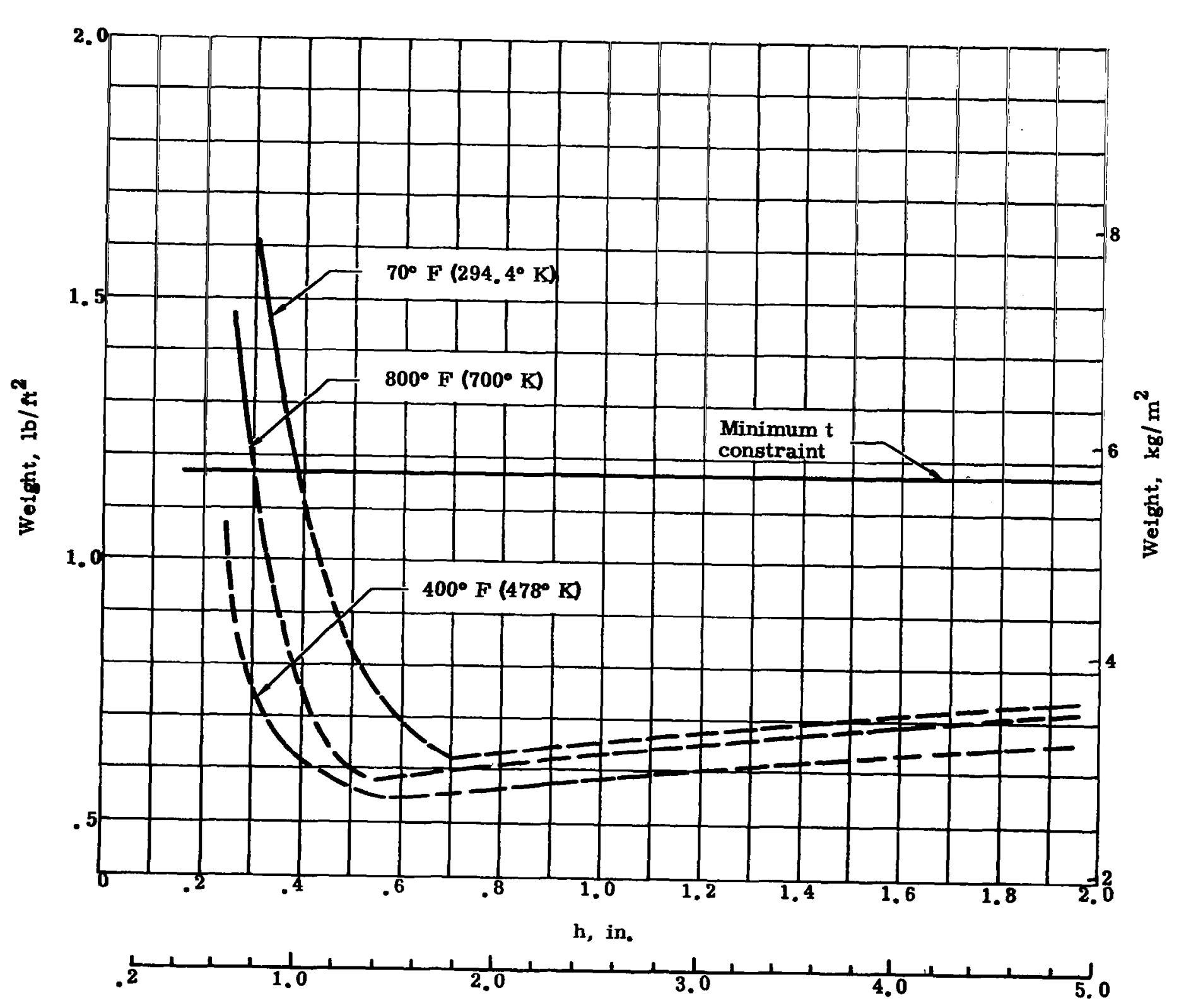


$\stackrel{\$}{\infty}$

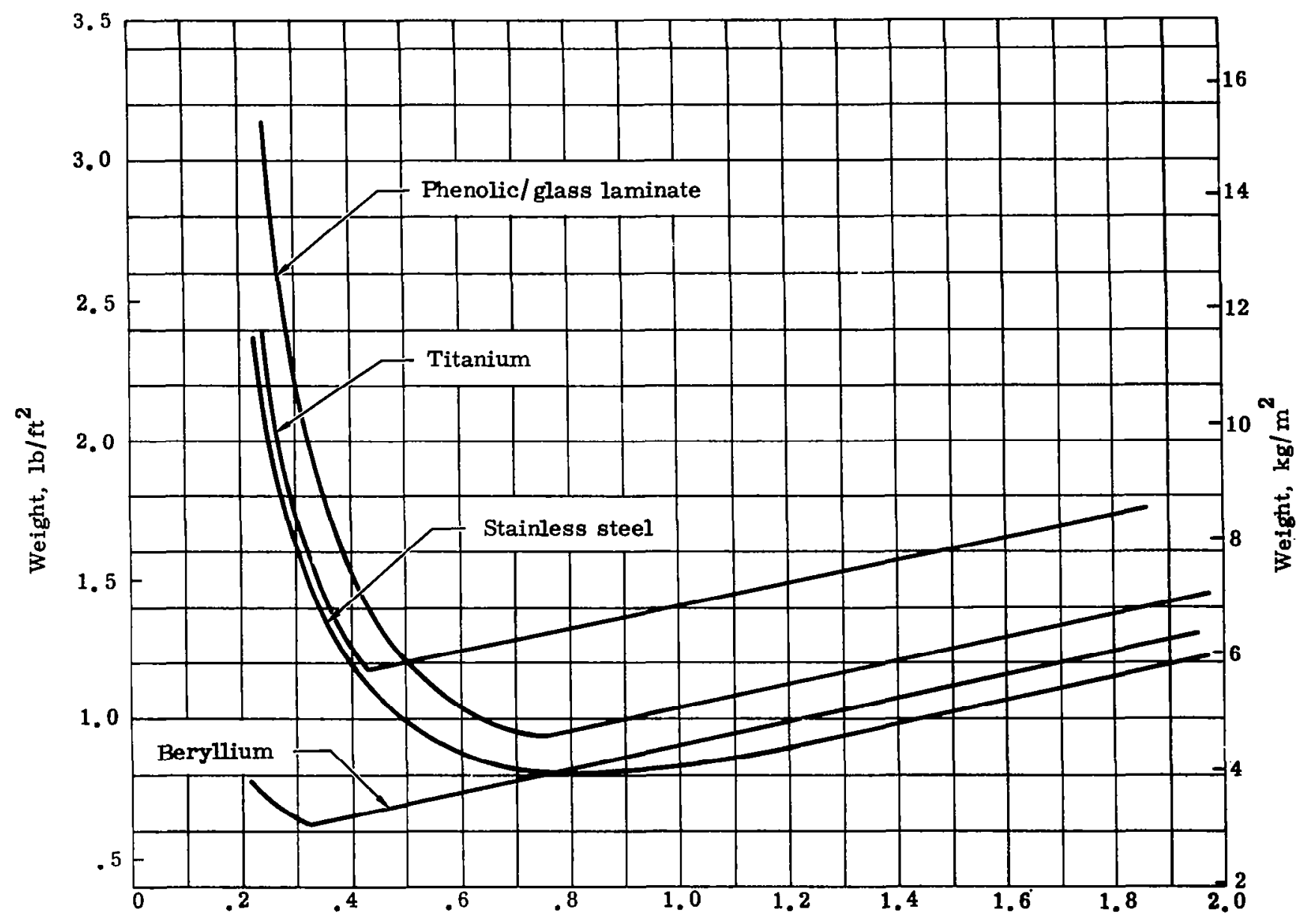

$h$, in.

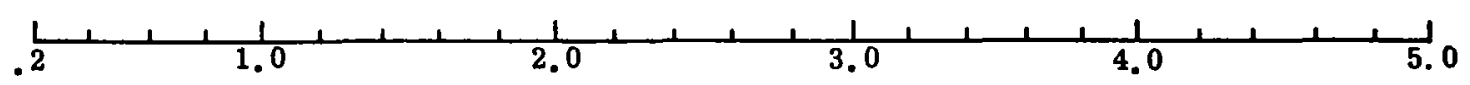

h, cm

FIGURE 40. OPTIMUM WEIGHT CURVES FOR HONEYCOMB PANELS AT ROOM TEMPERATURE 


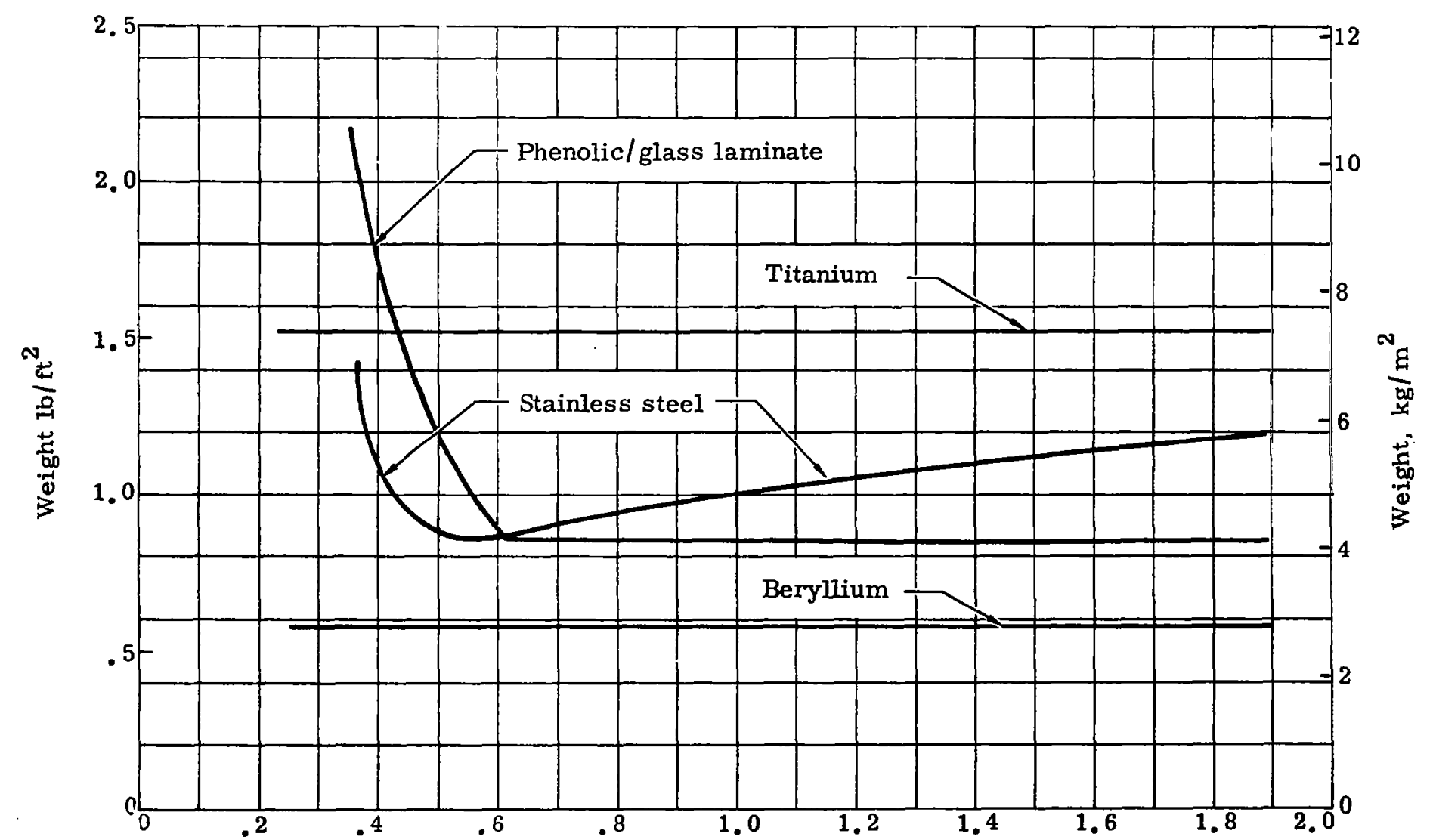

$h$, in.

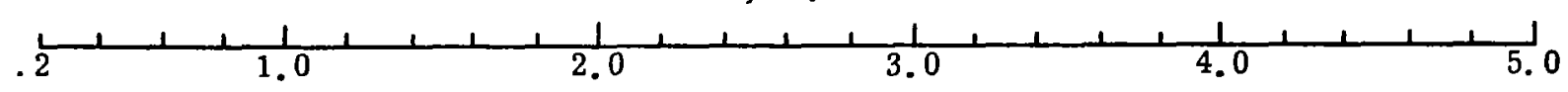

h, cm

FIGURE 41. OPTIMUM WEIGHT CURVES FOR SINGLE SKIN CORRUGATION PANELS AT ROOM TEMPERATURE 


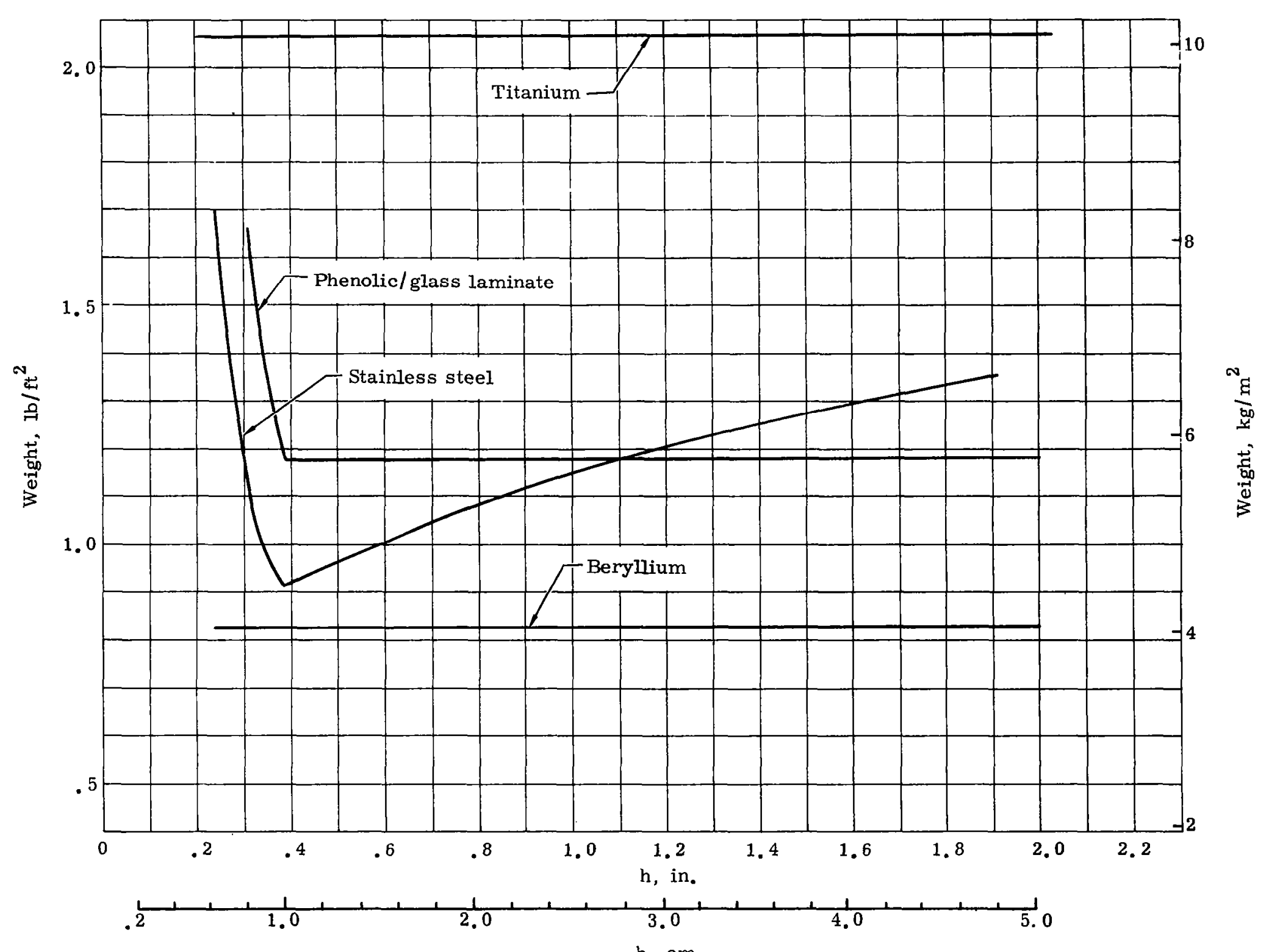

FIGURE 42. OPTIMUM WEIGHT CURVES FOR DOUBLE SKIN CORRUGATION PANELS AT ROOM TEMPERATURE 


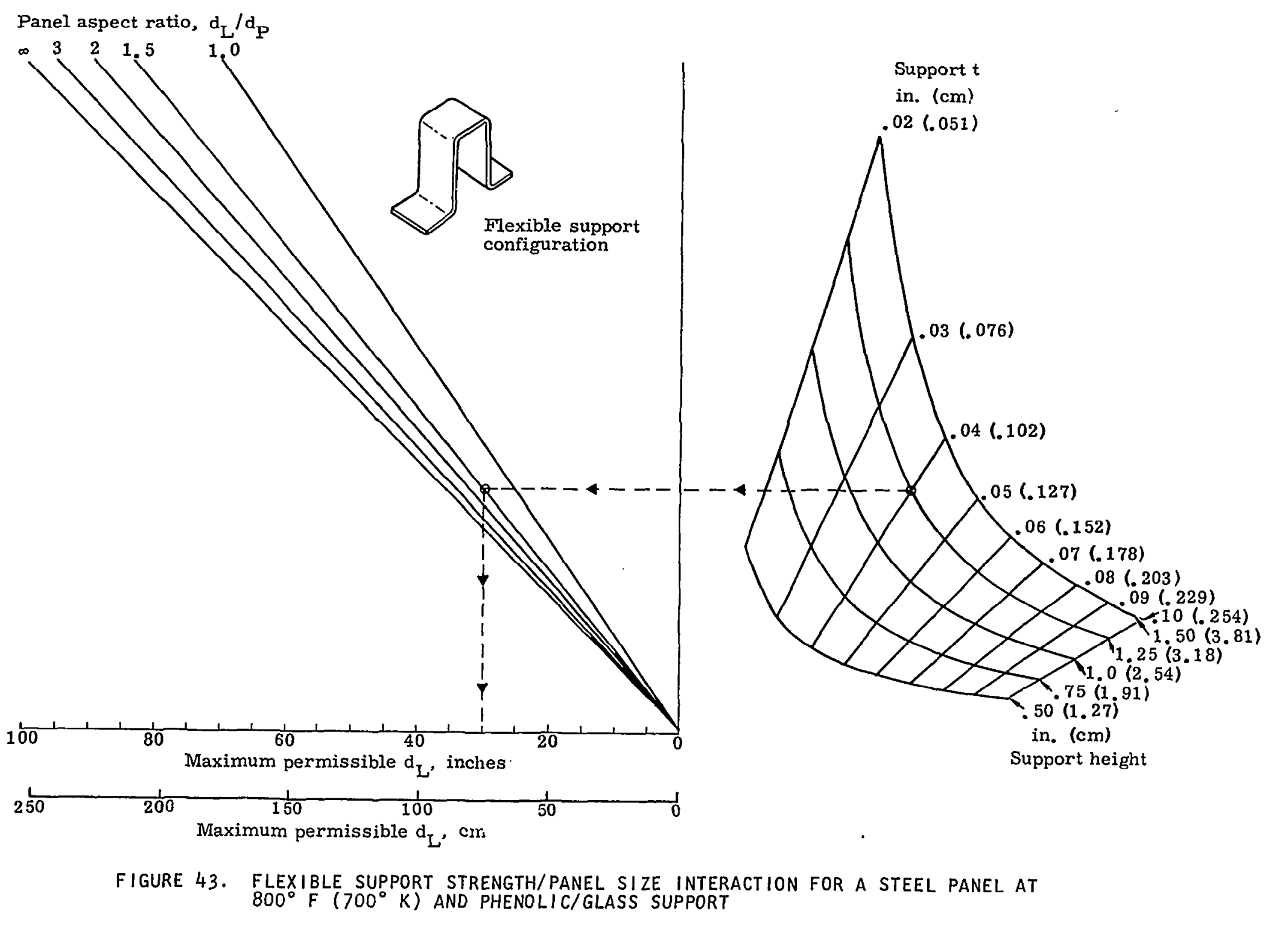




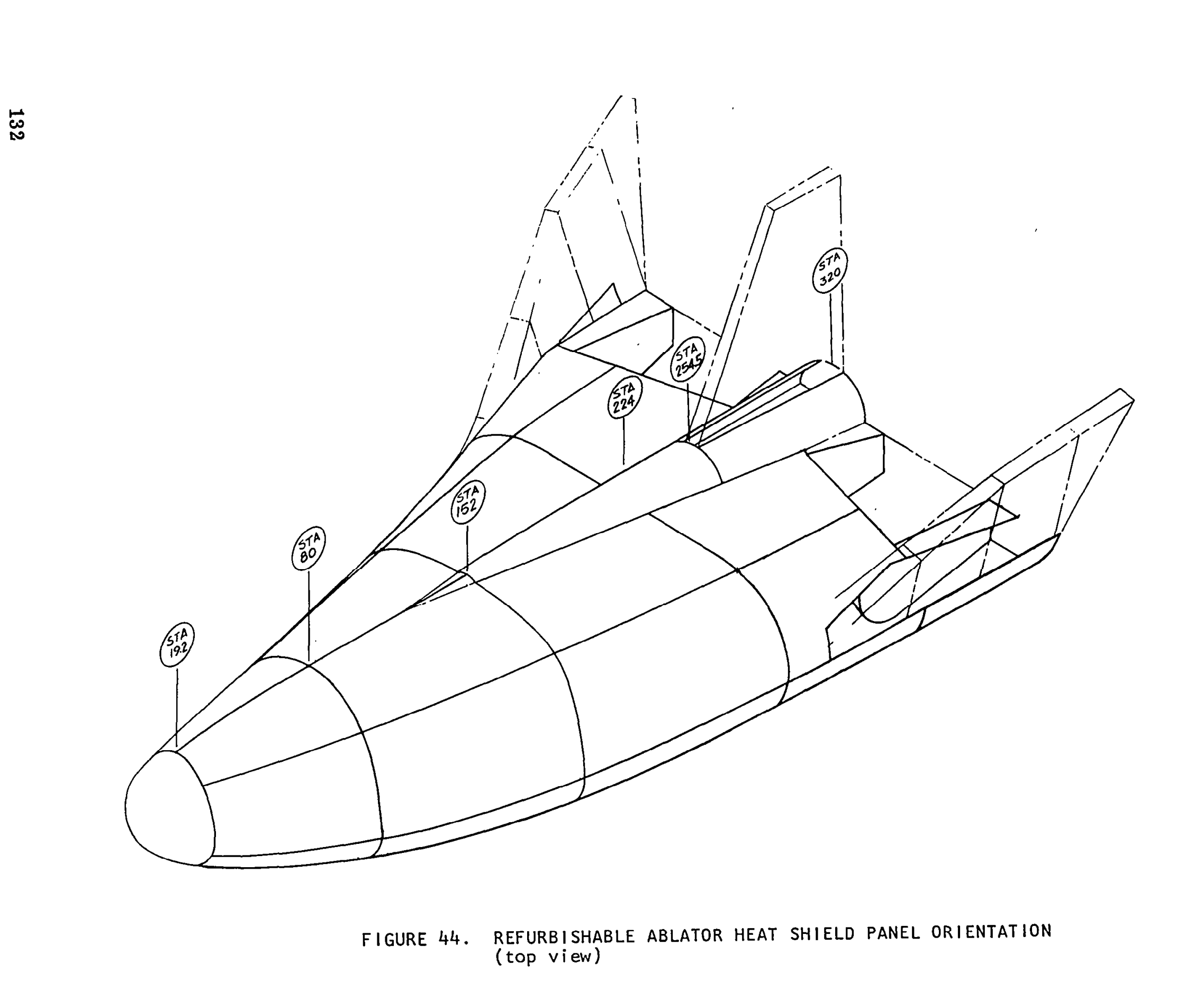




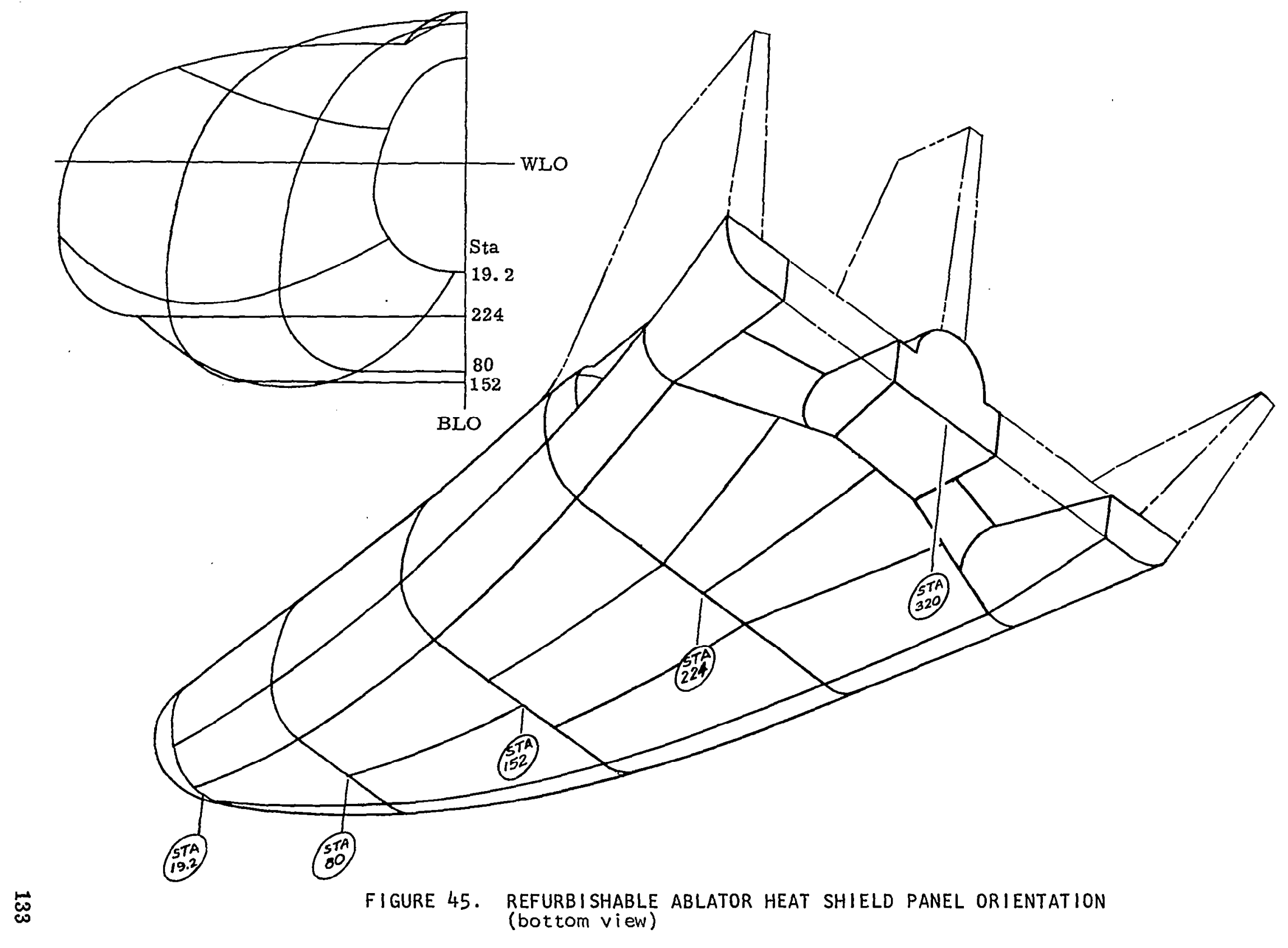




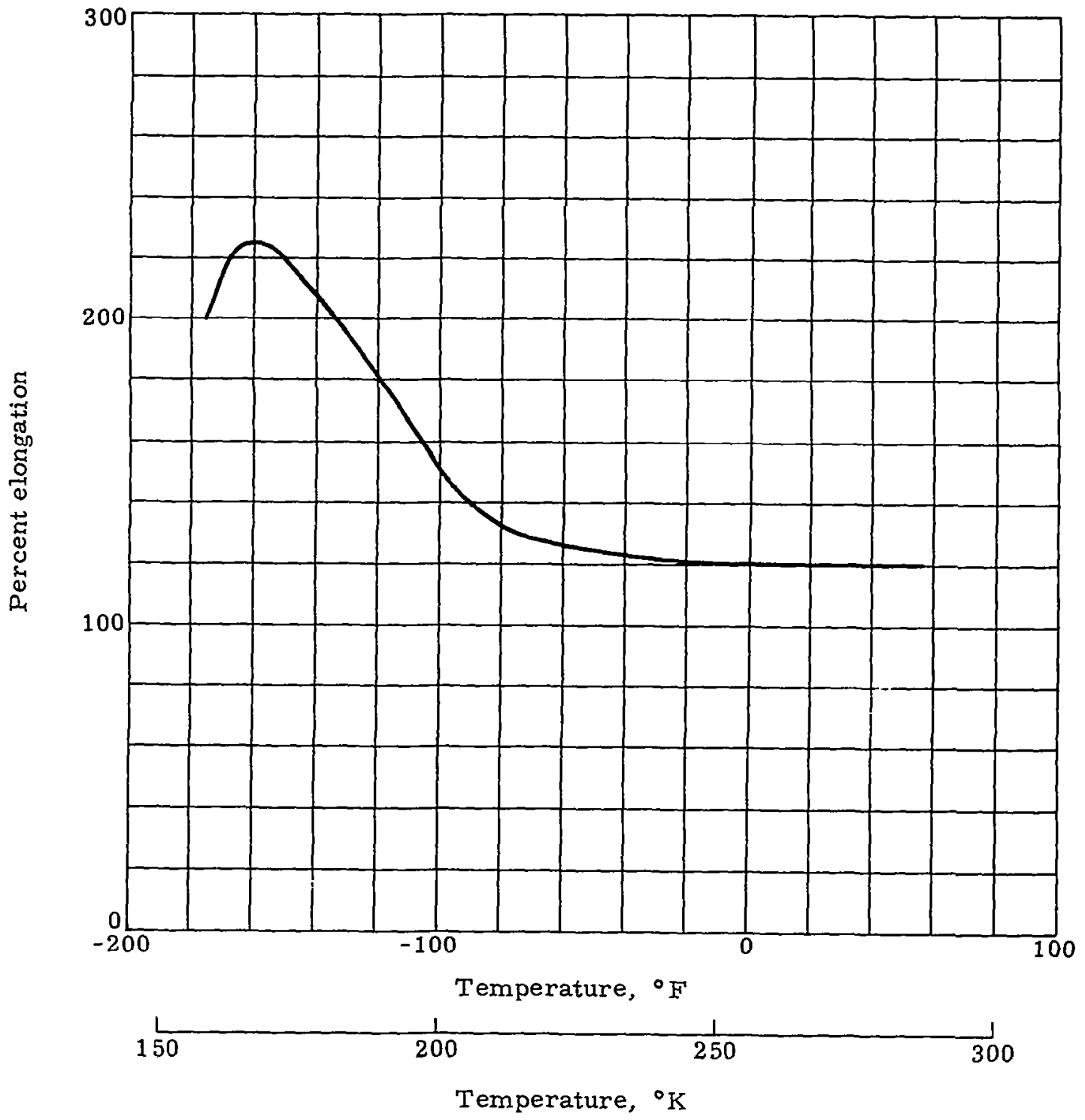

FIGURE 46. RTV 560 SILICON RUBBER LOW TEMPERATURE ULTIMATE 


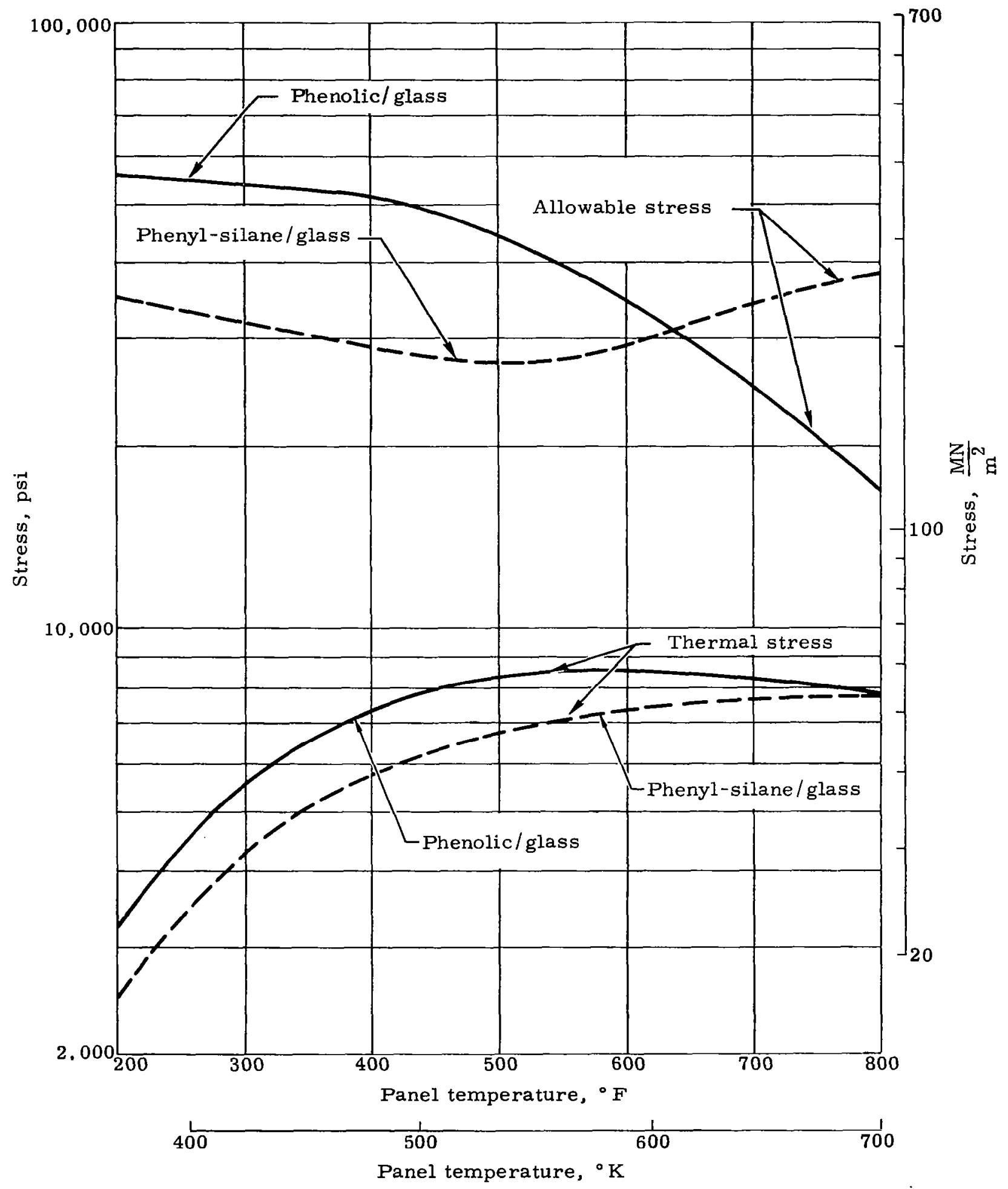

FIGURE 47. THERMAL STRESSES IN RIGIDLY SUPPORTED PLASTIC LAMINATE SUBSTRATE PANELS 


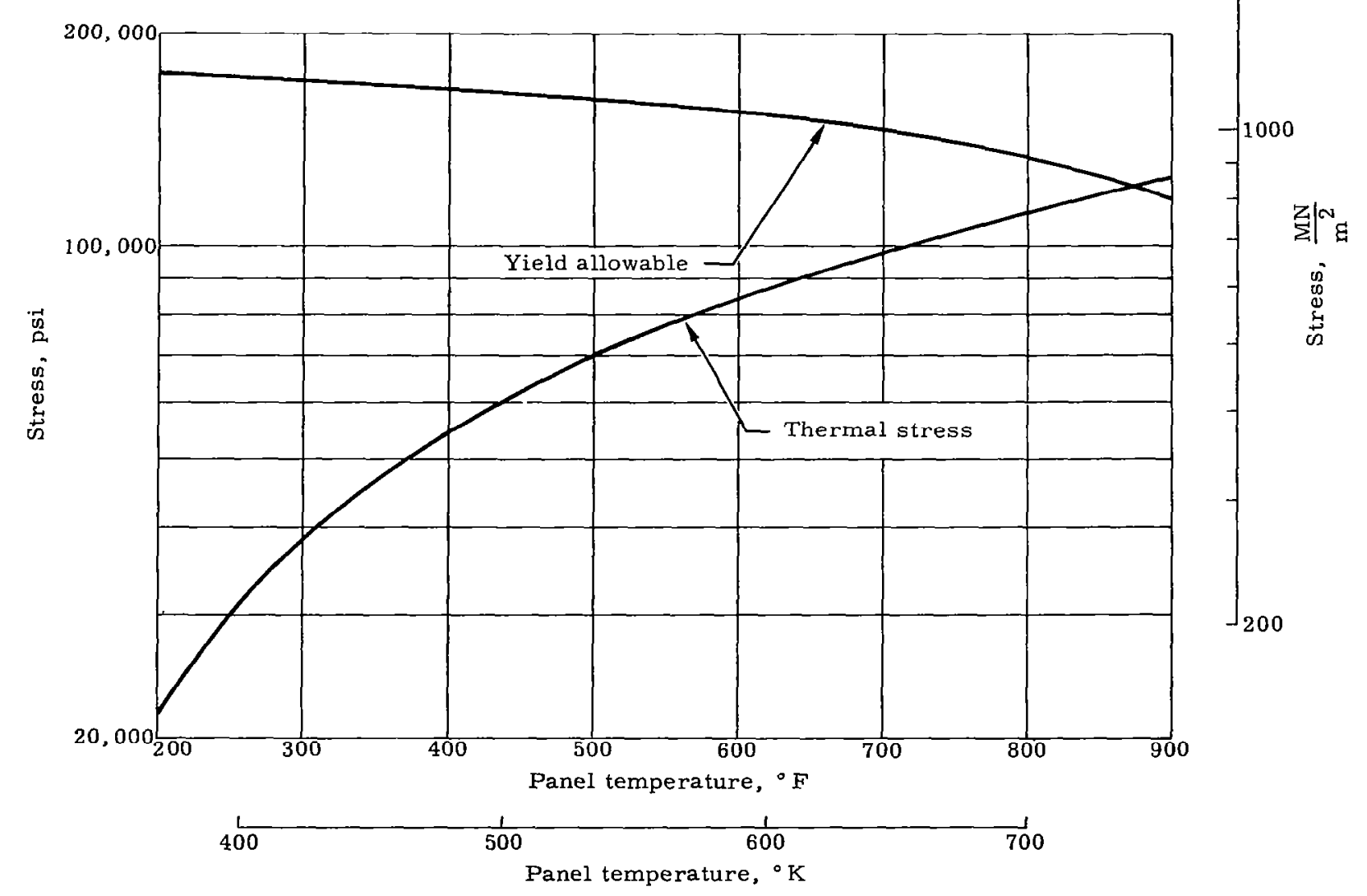

FIGURE 48. THERMAL STRESSES IN RIGIDLY SUPPORTED STAINLESS STEEL SUBSTRATE PANELS 


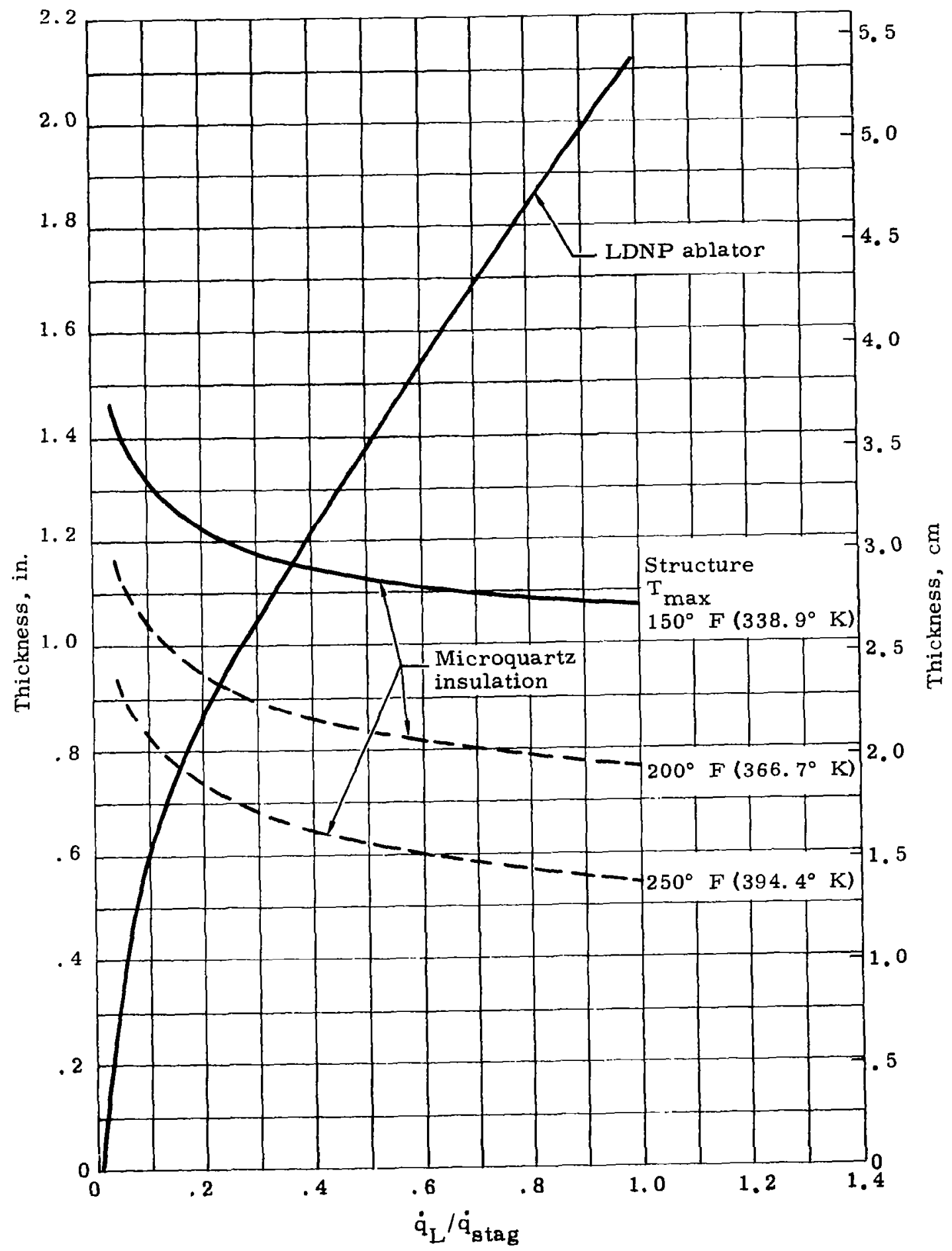

FIGURE 49. HEAT SHIELD DESIGN CURVE (NOMINAL TRAJECTORY, LDNP ABLATOR) 


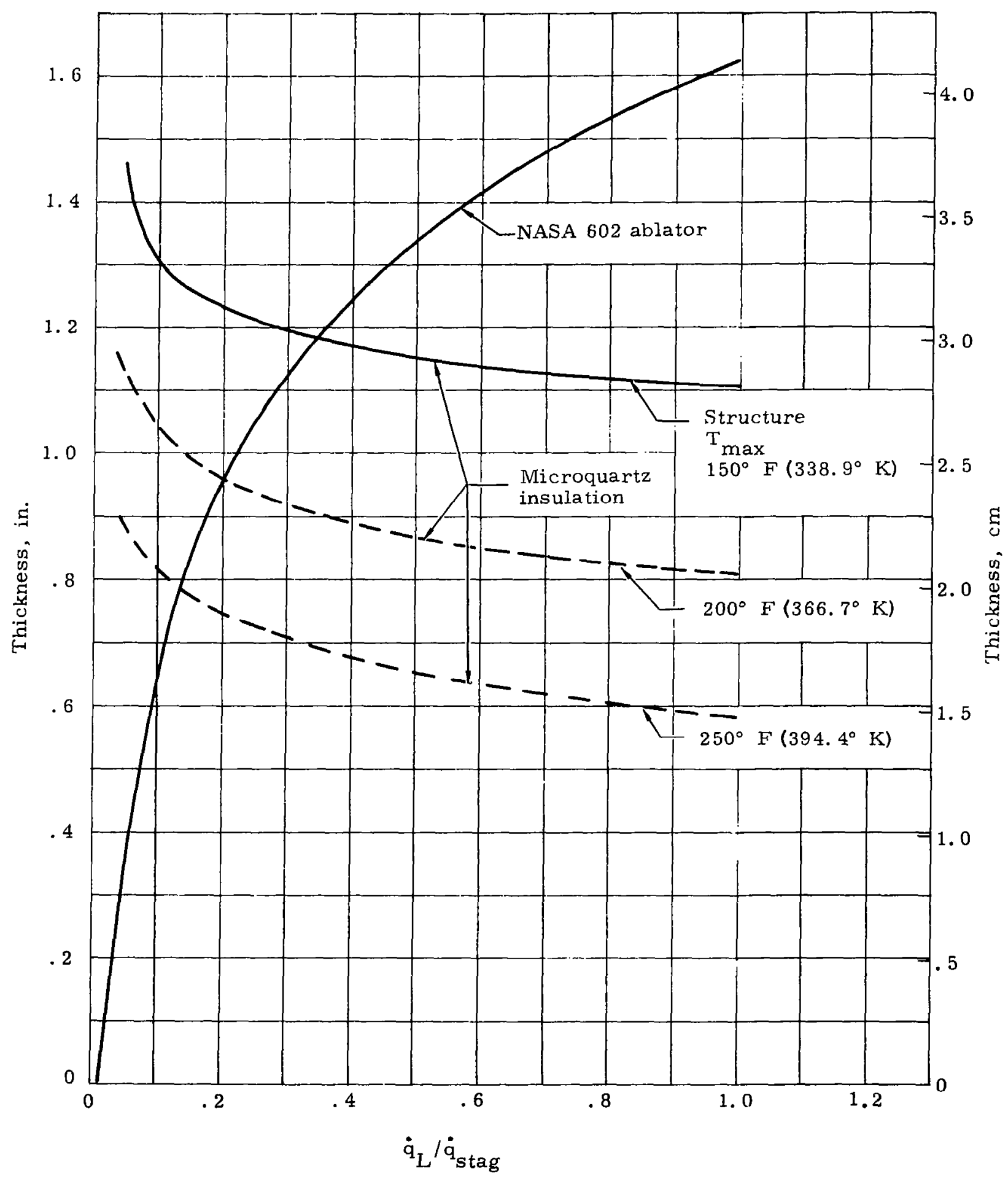

FIGURE 50. HEAT SHIELD DESIGN CURVE (NOMINAL TRAJECTORY, NASA 602 ABLATOR) 


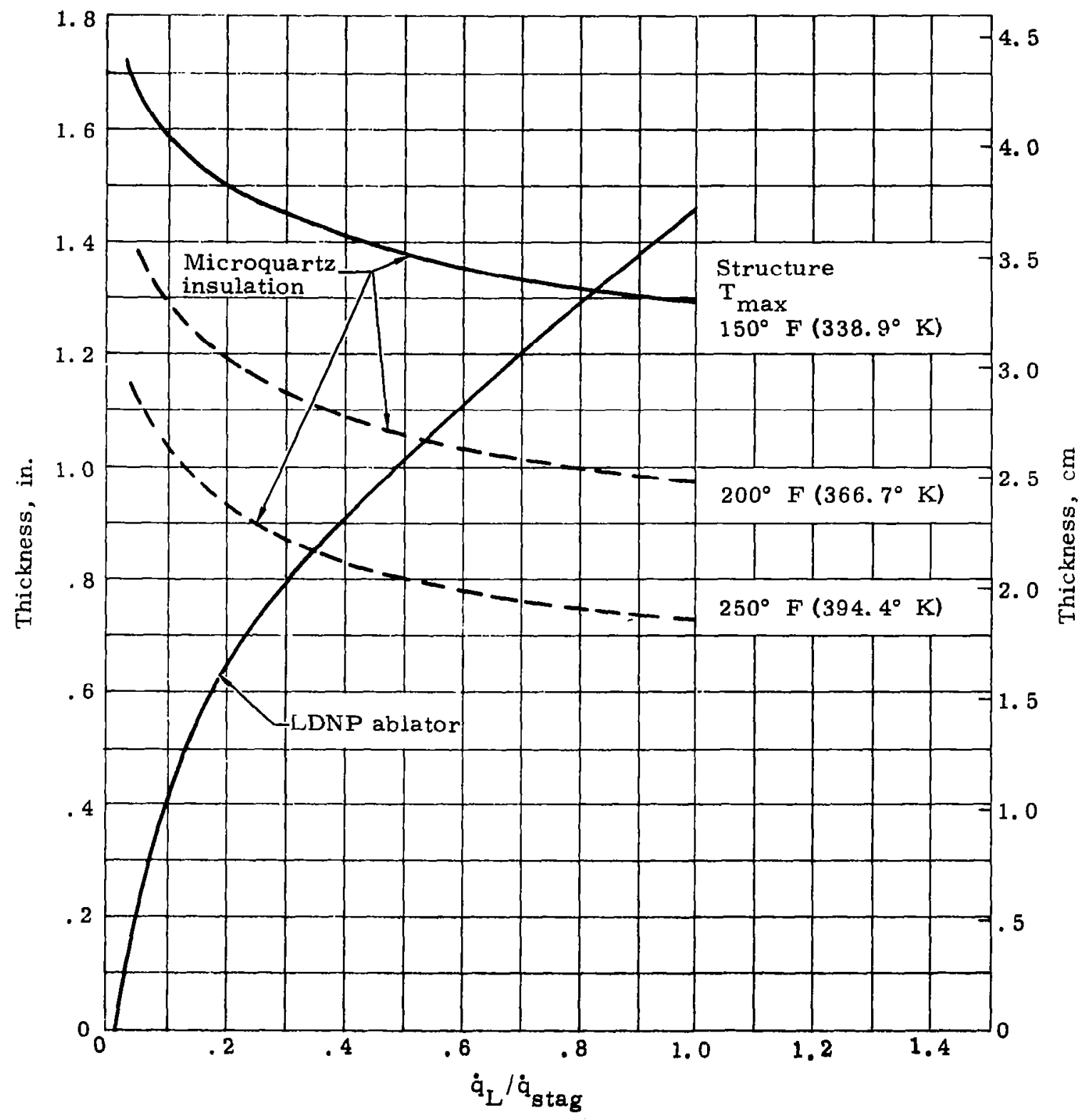

FIGURE 51. HEAT SHIELD DESIGN CURVE (OVERSHOOT TRAJECTORY, LDNP ABLATOR) 


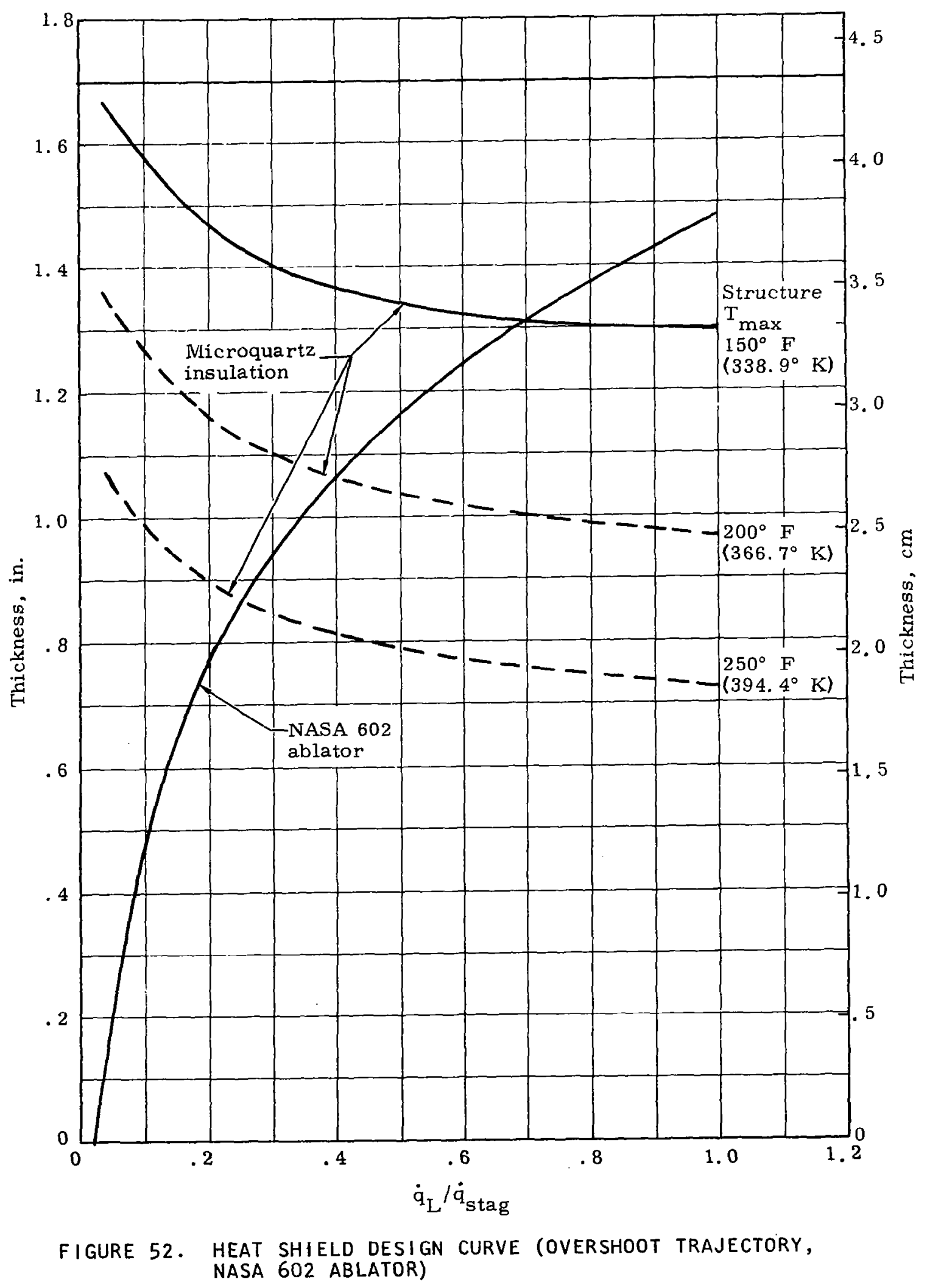


-- LDNP

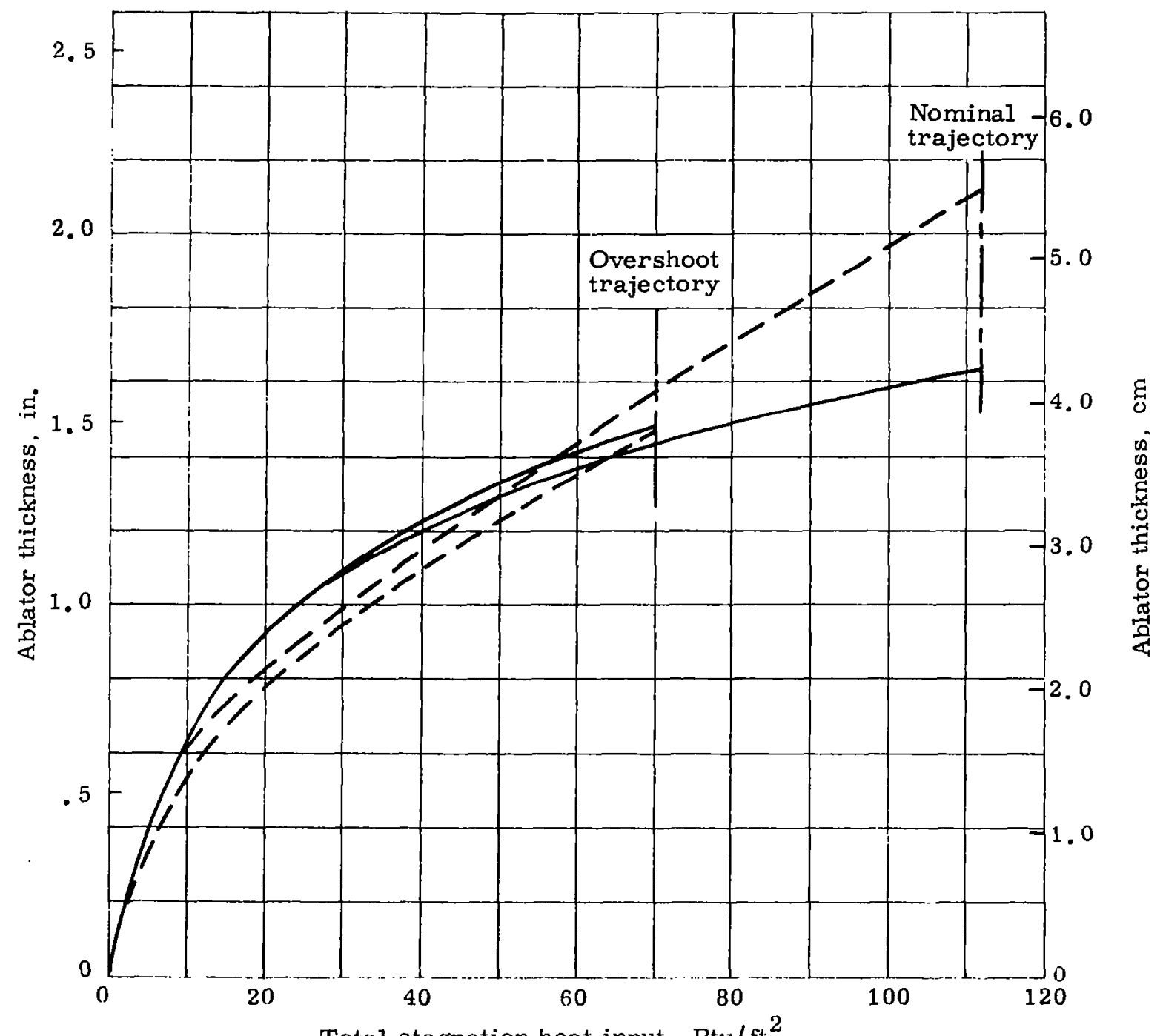

Total stagnation heat input, Btu/ $\mathrm{ft}^{2}$

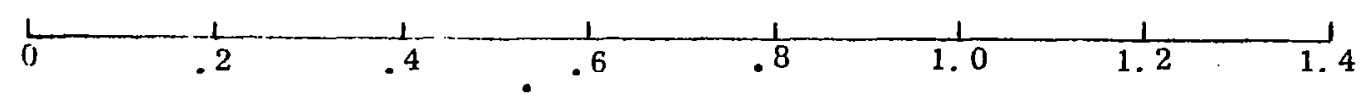

Total stagnation heat input, $\mathrm{MJ} / \mathrm{m}^{2}$

FIGURE 53. COMPARISON OF ABLATOR THICKNESS DESIGN CURVES ON A TOTAL HEAT BASIS 


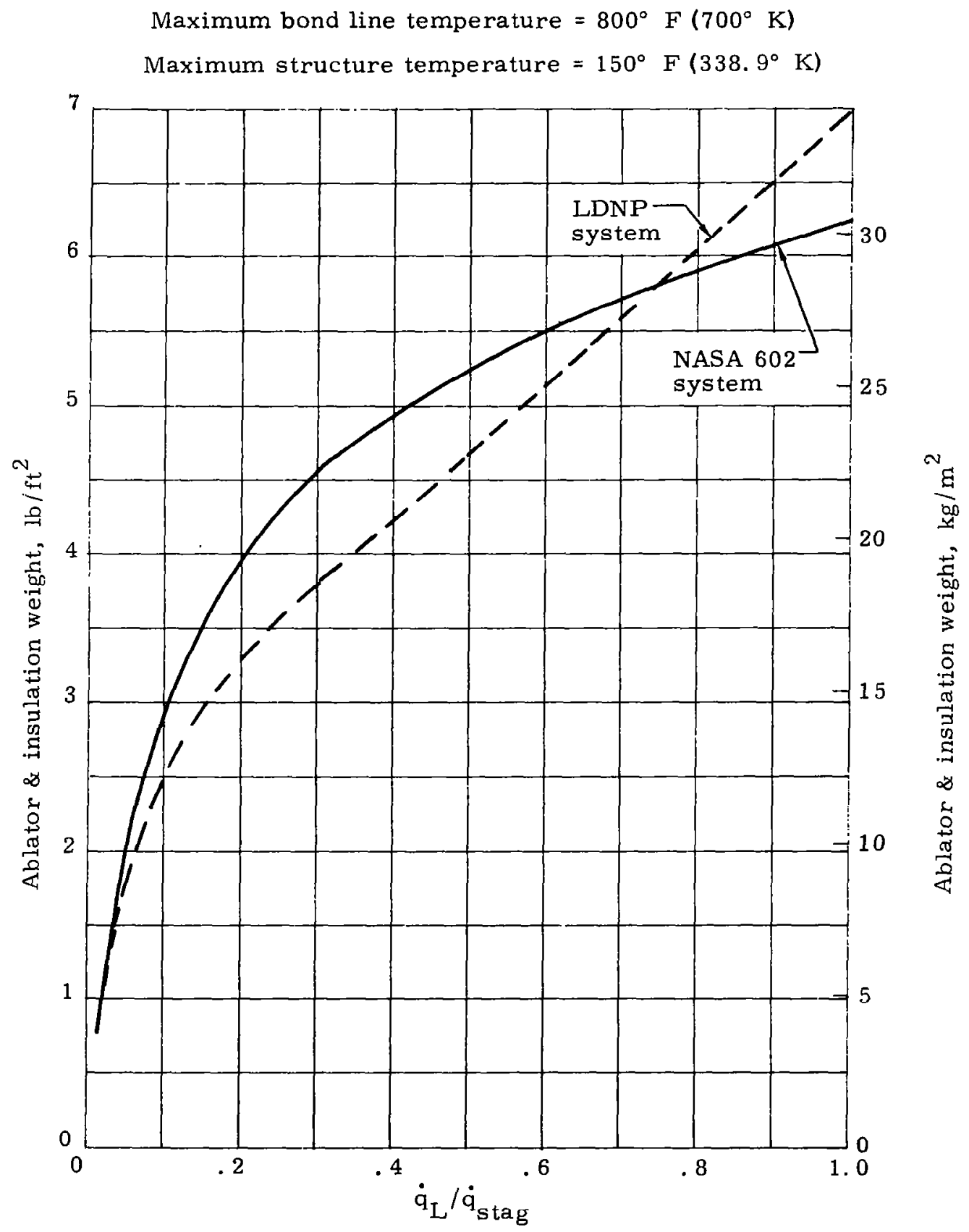

FIGURE 54. HEAT SHIELD WEIGHT CURVE (NOMINAL RE-ENTRY TRAJECTORY) 


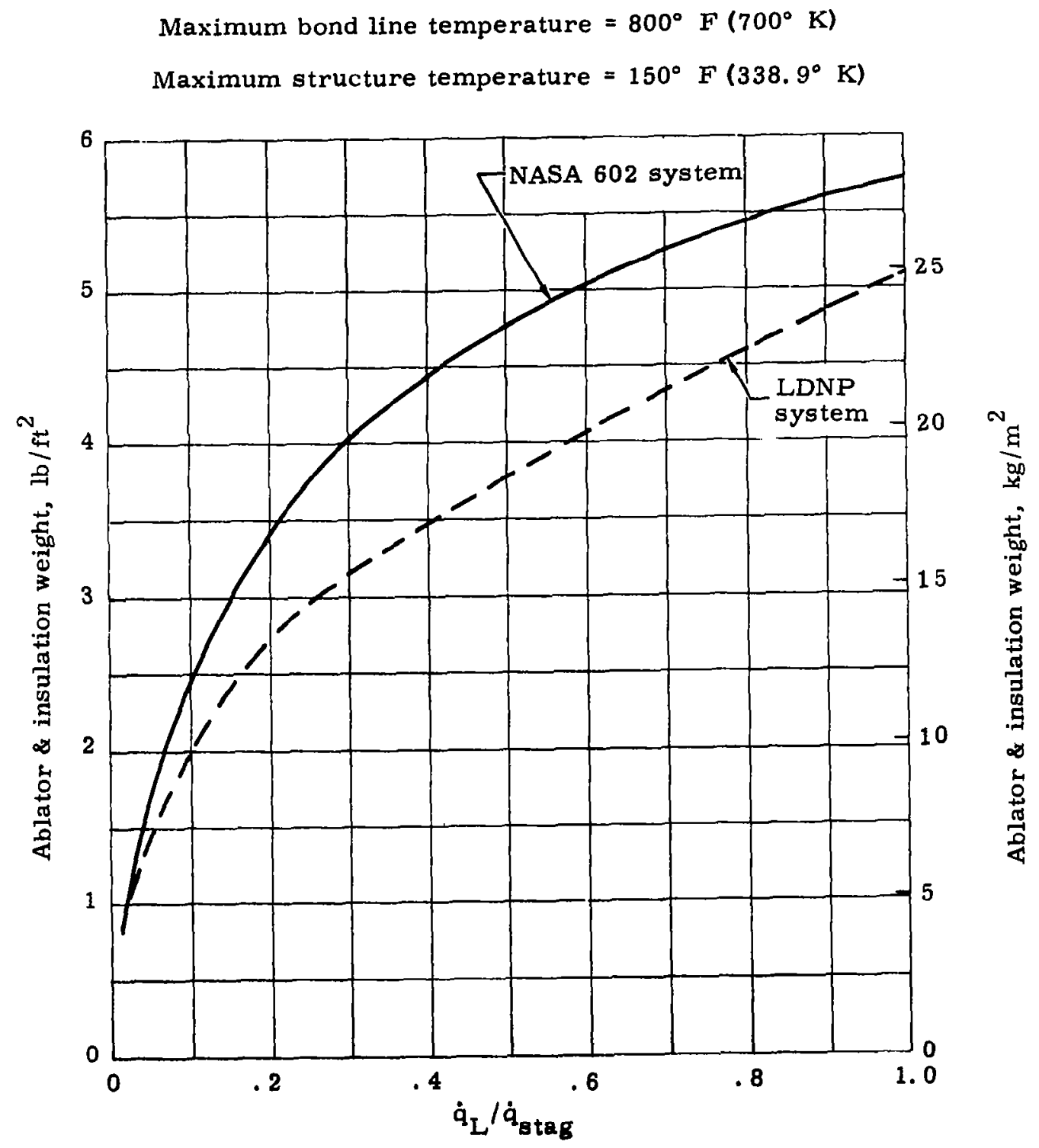

FIGURE 55. HEAT SHIELD WEIGHT CURVE (OVERSHOOT RE-ENTRY TRAJECTORY) 

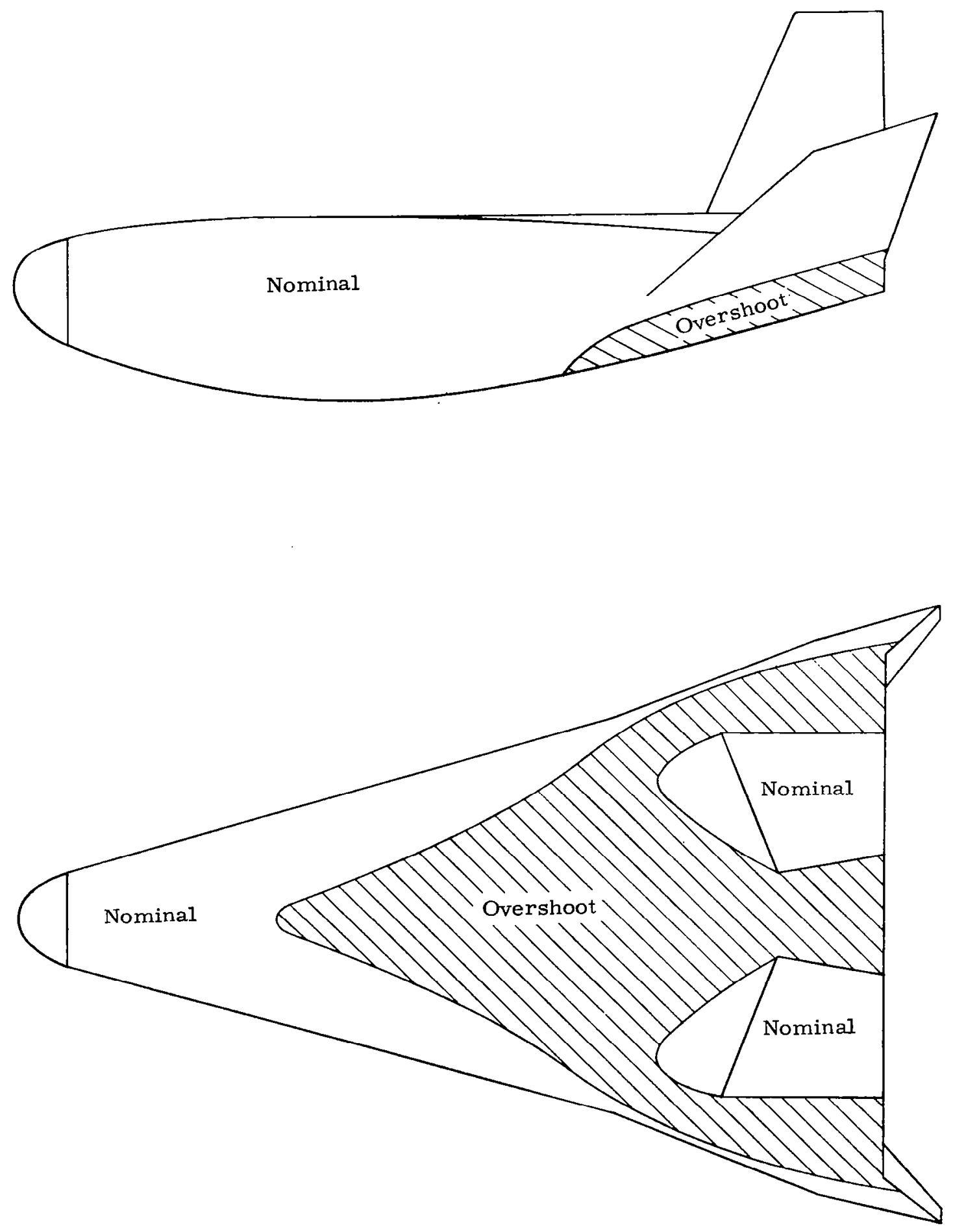

FIGURE 56. CRITICAL TRAJECTORIES FOR ABLATOR THICKNESS DETERMINATION (low density nylon phenolic) 

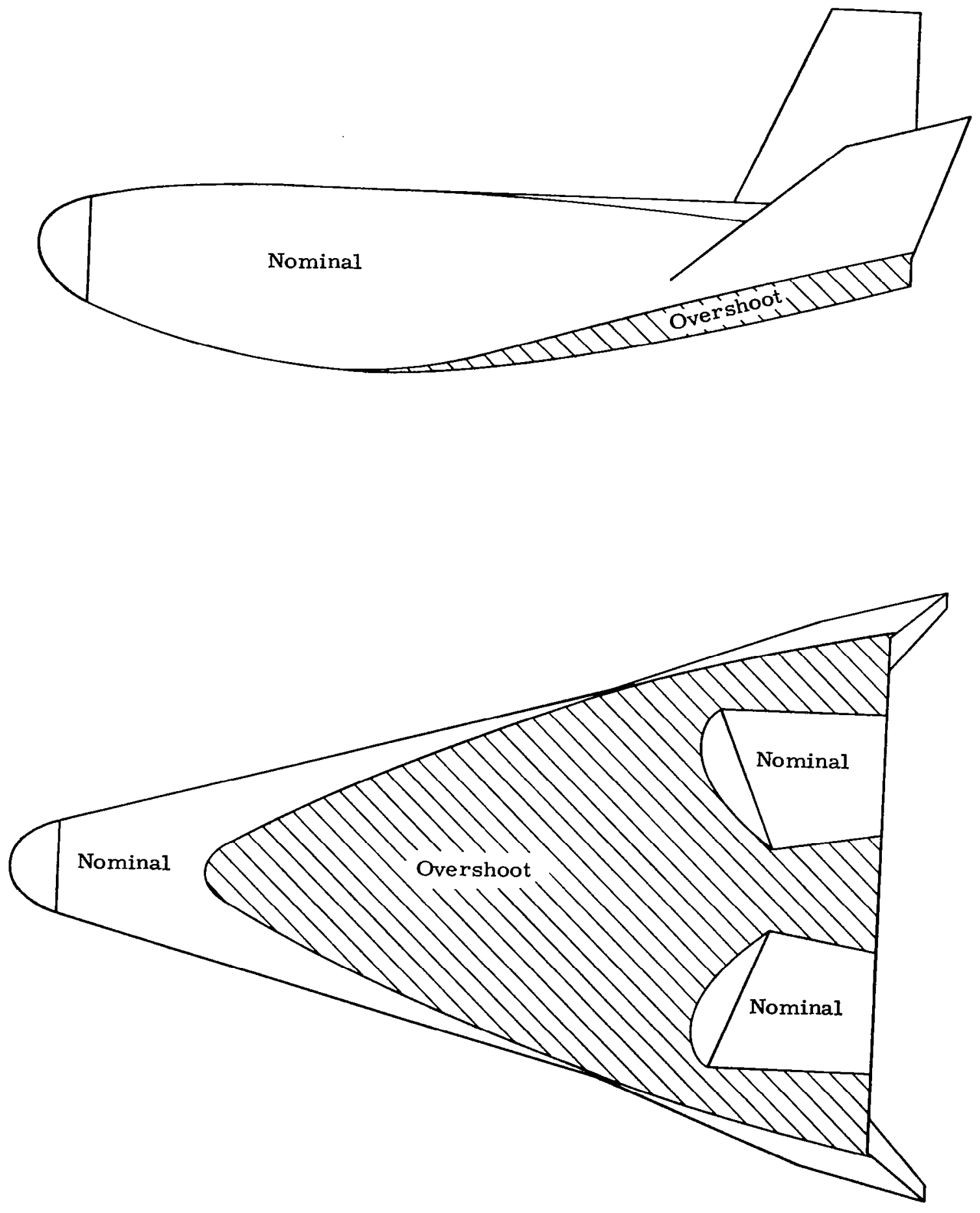

FIGURE 57. CRITICAL TRAJECTORIES FOR ABLATOR THICKNESS DETERMINATION (NASA 602) 


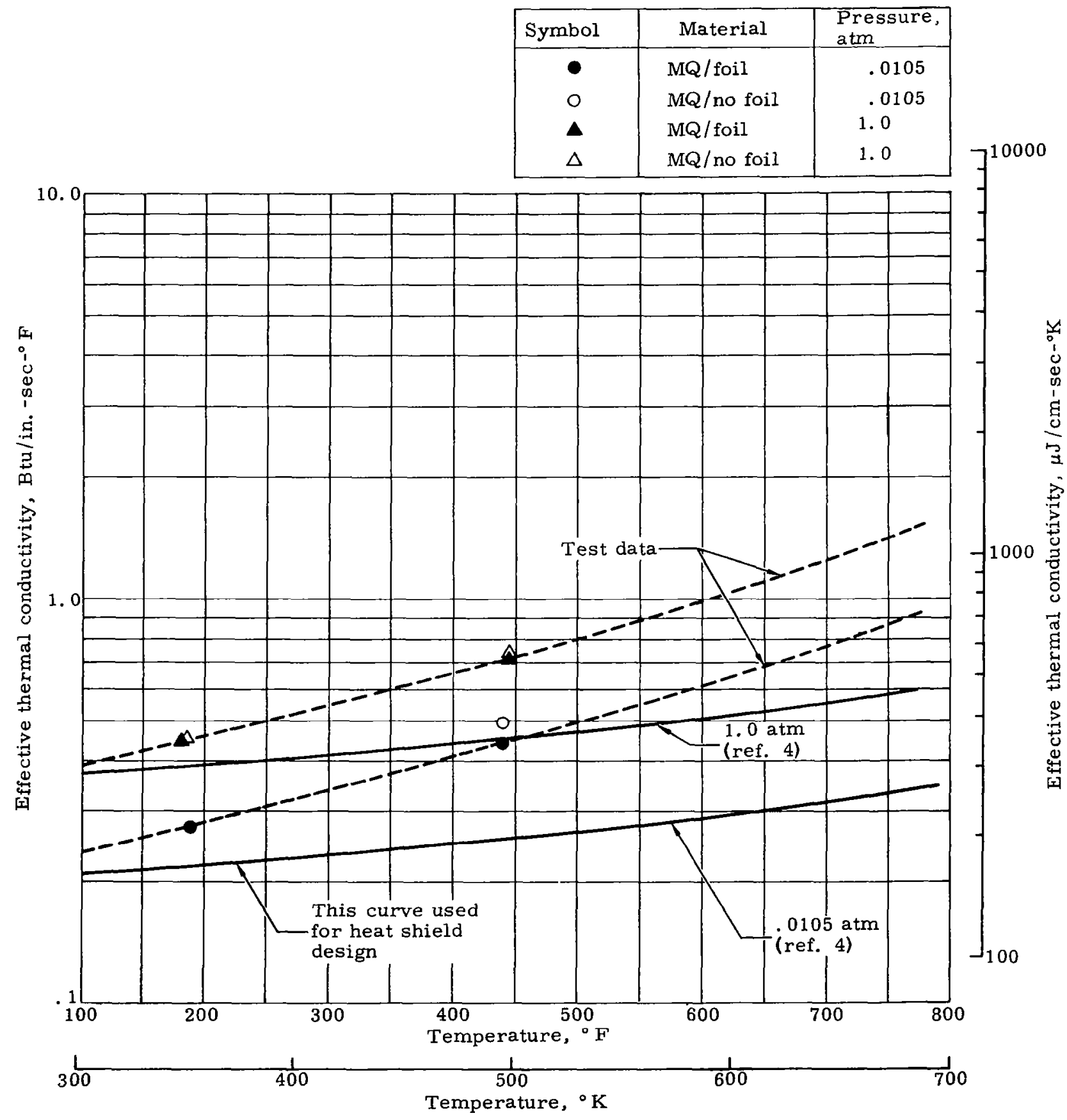

FIGURE 58. COMPARISON OF MICROQUARTZ THERMAL CONDUCTIVITY VALUES 


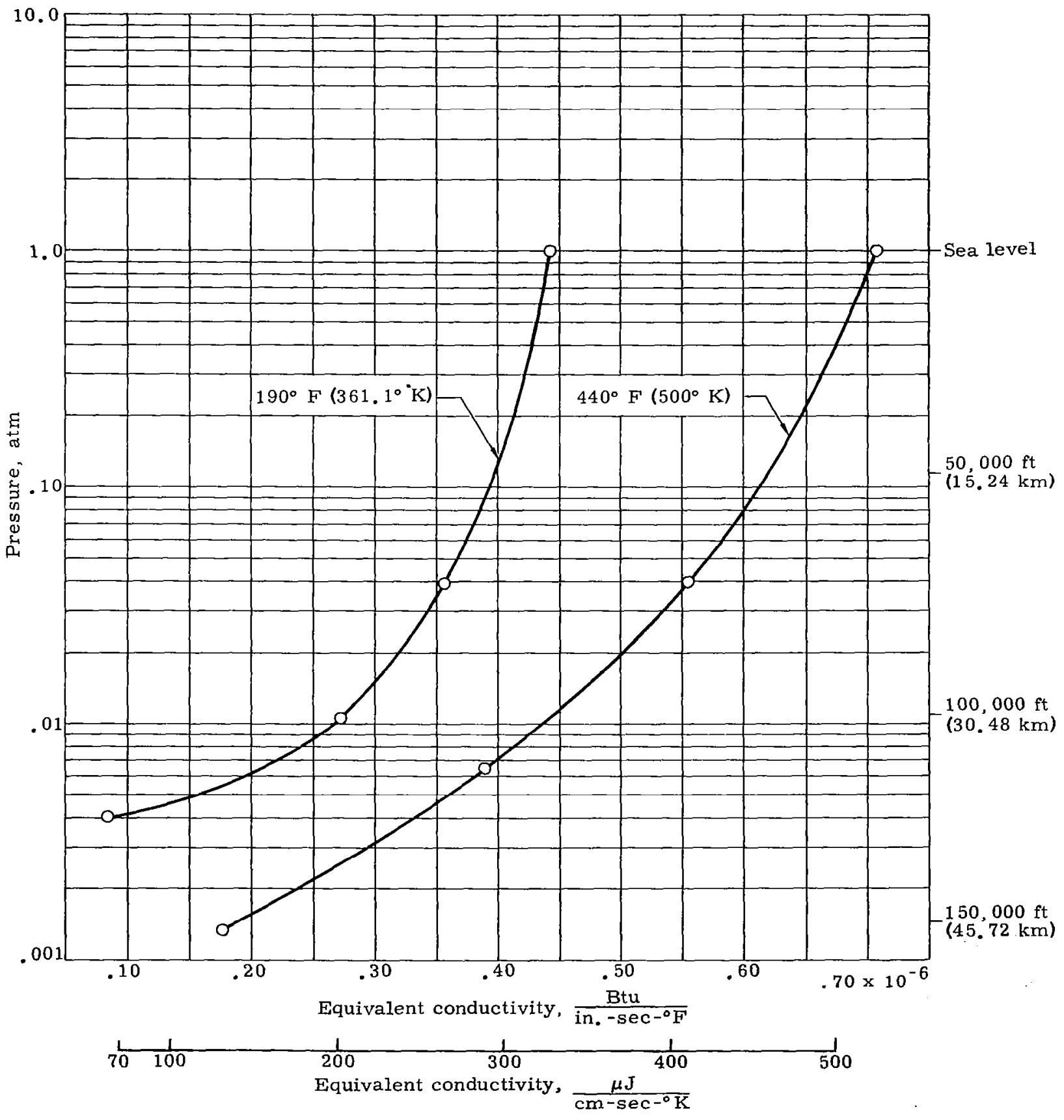

FIGURE 59. PRESSURE DEPENDENCE OF MICROQUARTZ/FOIL

INSULATION THERMAL CONDUCTIVITY AT TWO

TEMPERATURES 

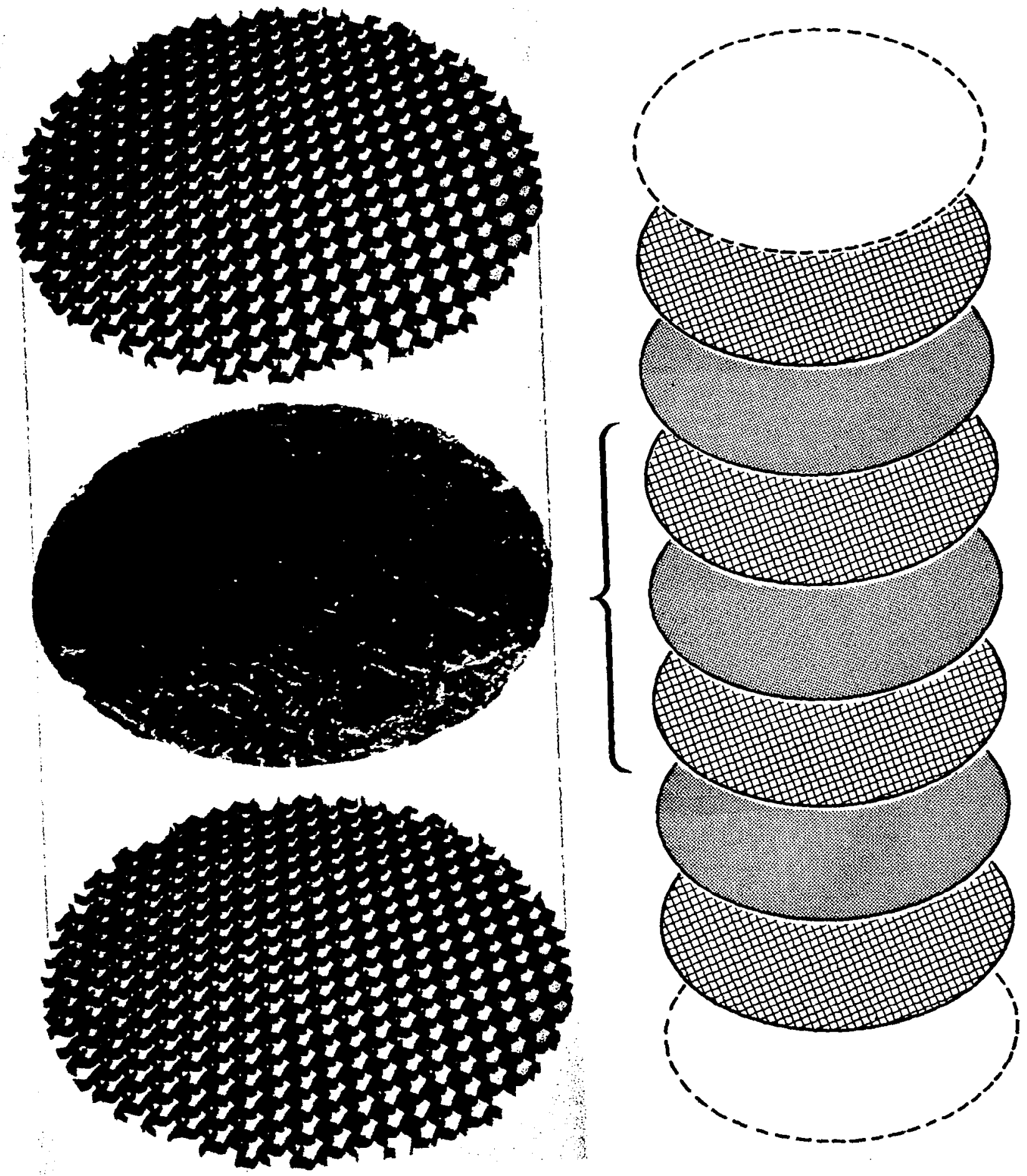

Typical construction

FIGURE 60. INTEGRAL INSULATION/SUPPORT CONCEPT TEST SPECIMEN 


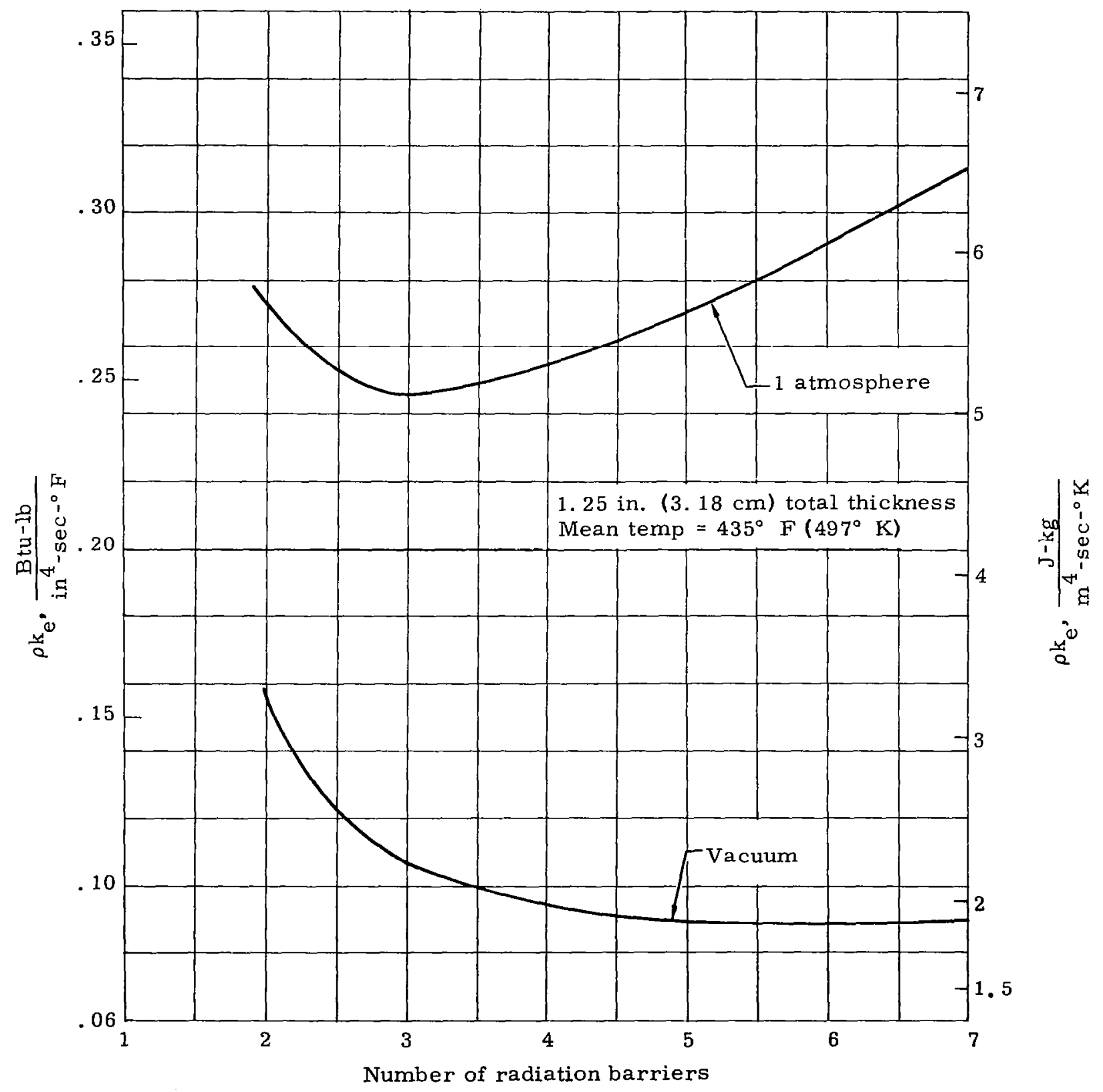

FIGURE 61. FLEXCORE HONEYCOMB/ALUMINUM FOIL INSULATION OPTIMIZATION 


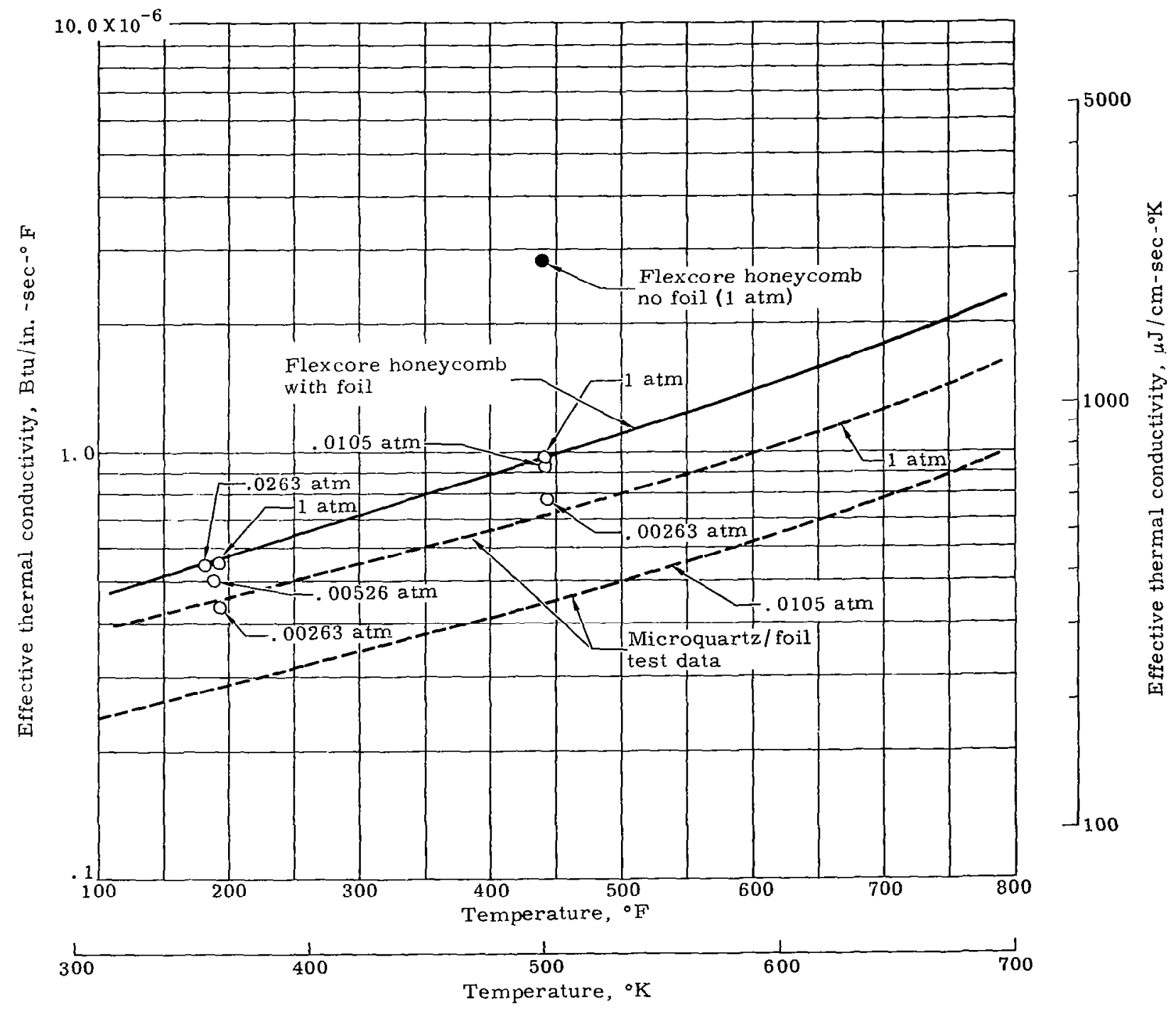

FIGURE 62. EFFECTIVE THERMAL CONDUCTIVITY VERSUS TEMPERATURE AND PRESSURE FOR FLEXCORE HONEYCOMB WITH ALUMINUM FOIL 


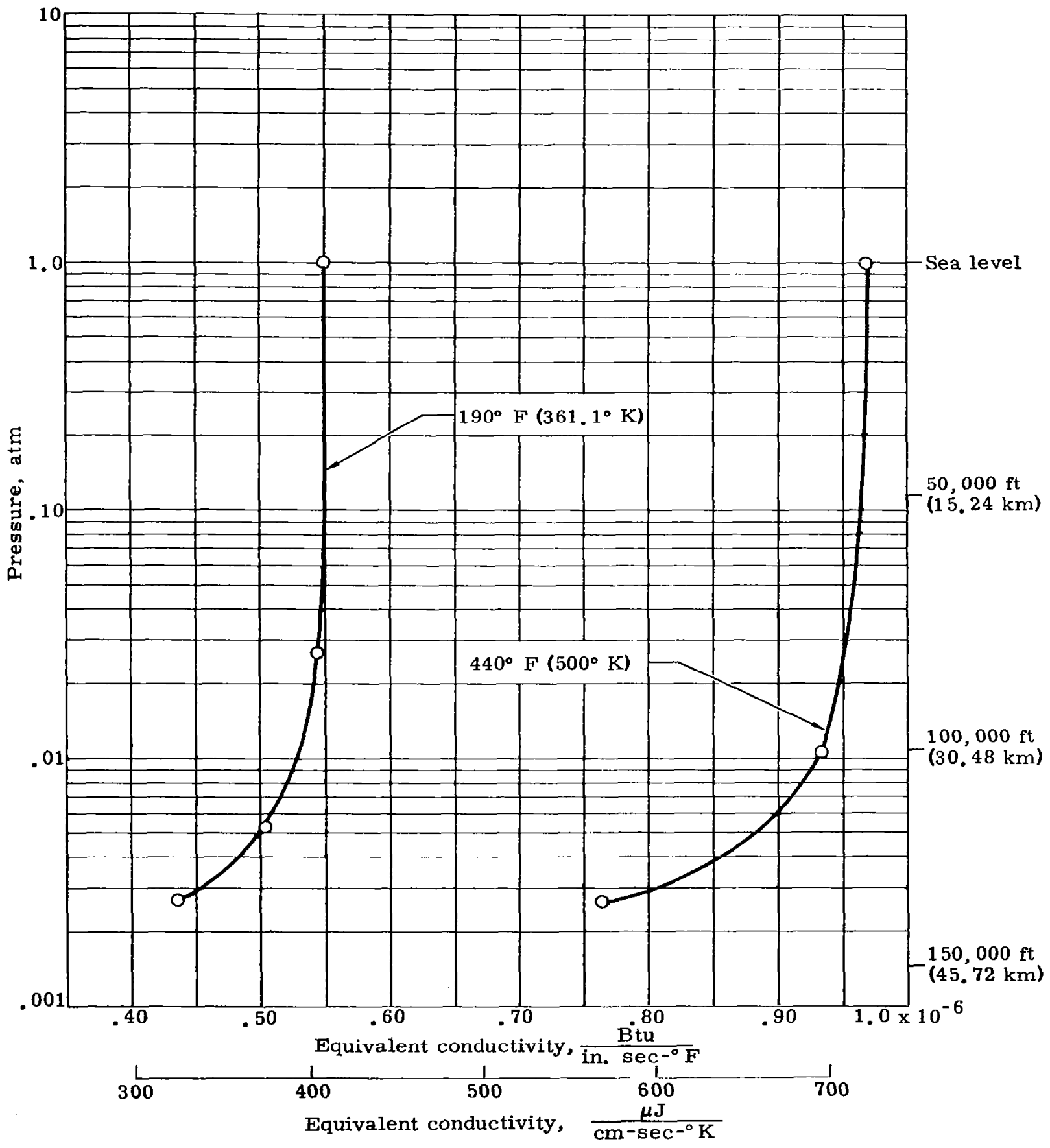

FIGURE 63. PRESSURE DEPENDENCE OF FLEXCORE HONEYCOMB WITH FOIL INSULATION THERMAL CONDUCTIVITY AT TWO TEMPERATURES 


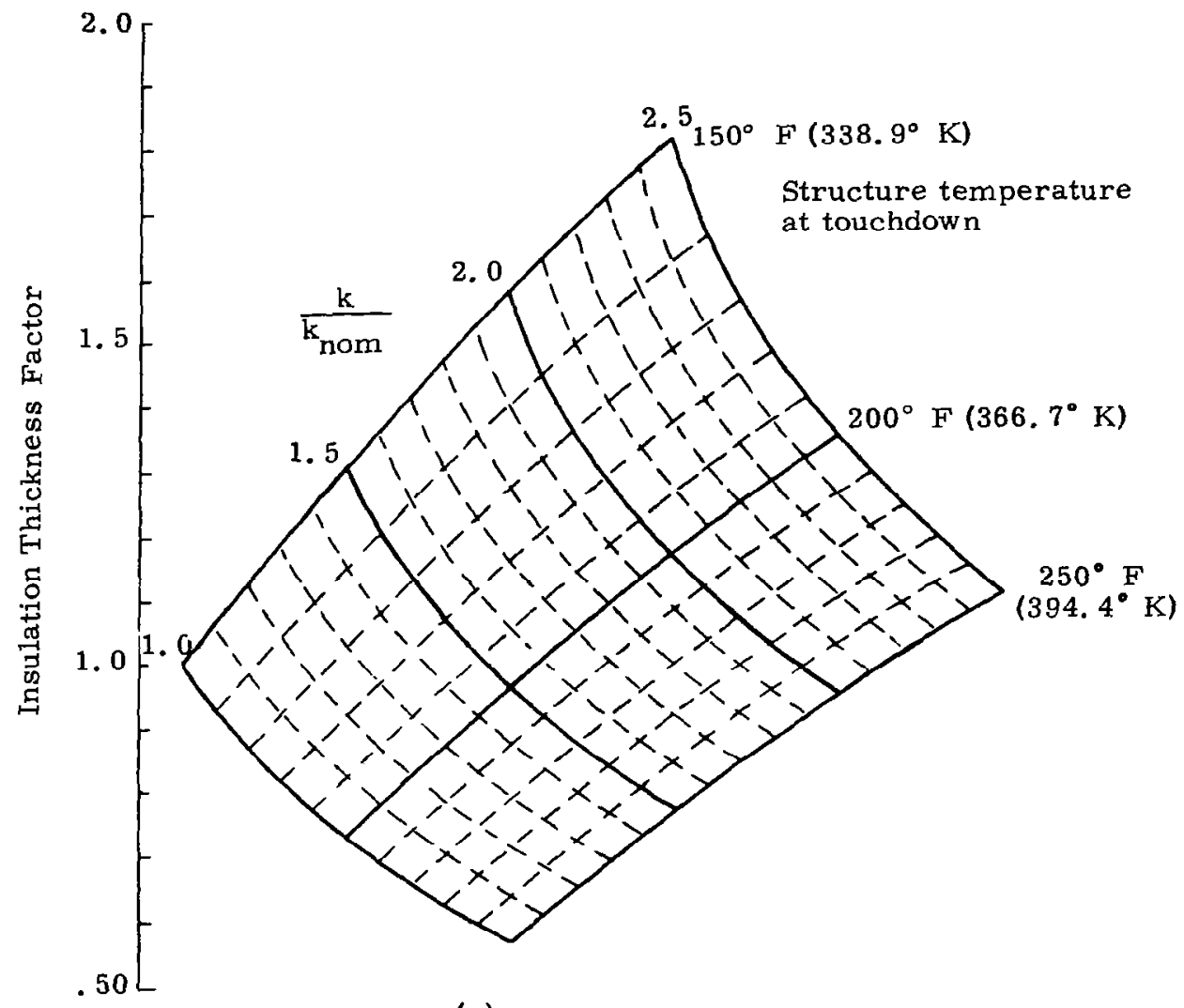

(a)

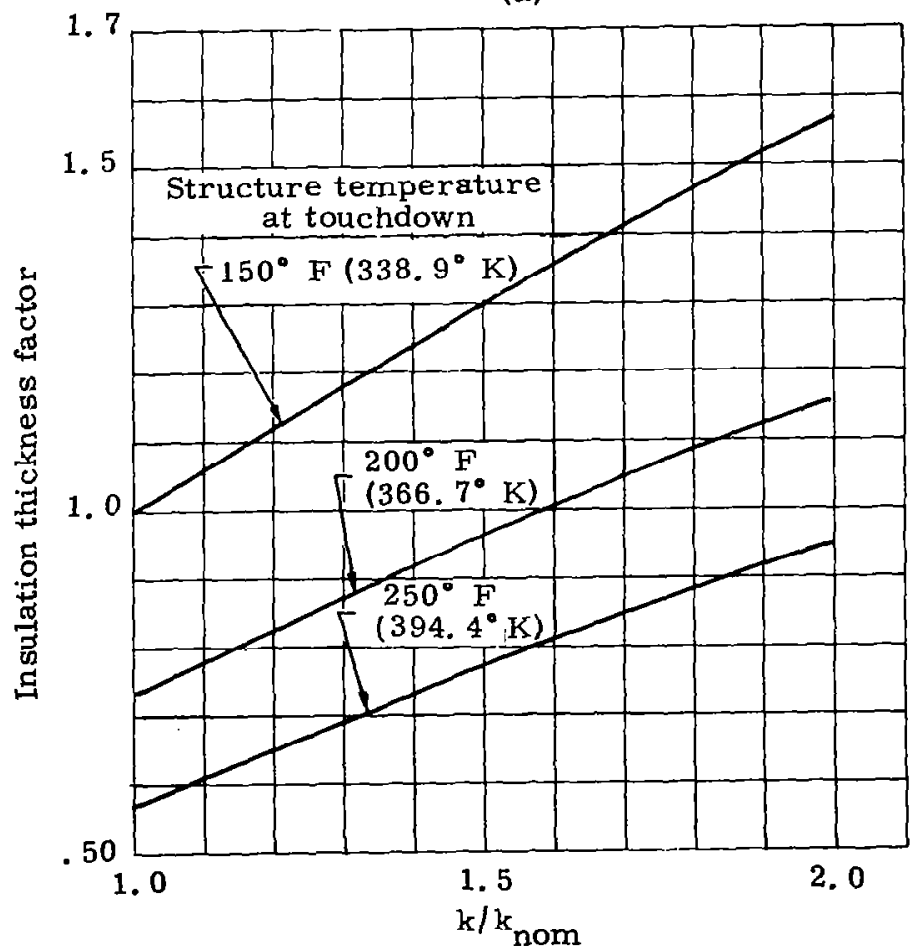

(b)

FIGURE 64. INSULATION CONDUCTIVITY/THICKNESS/STRUCTURE TEMPERATURE INTERACTION 


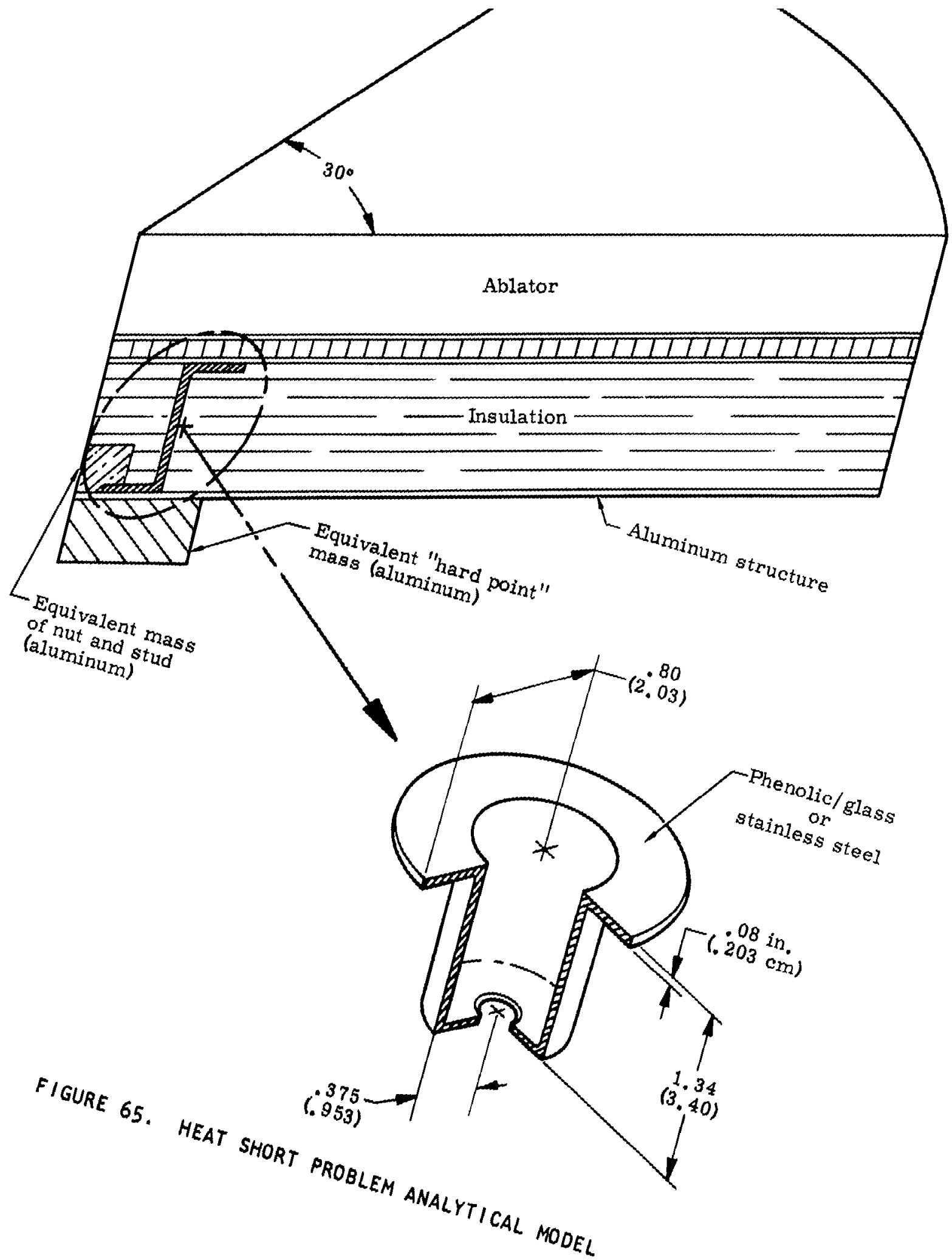




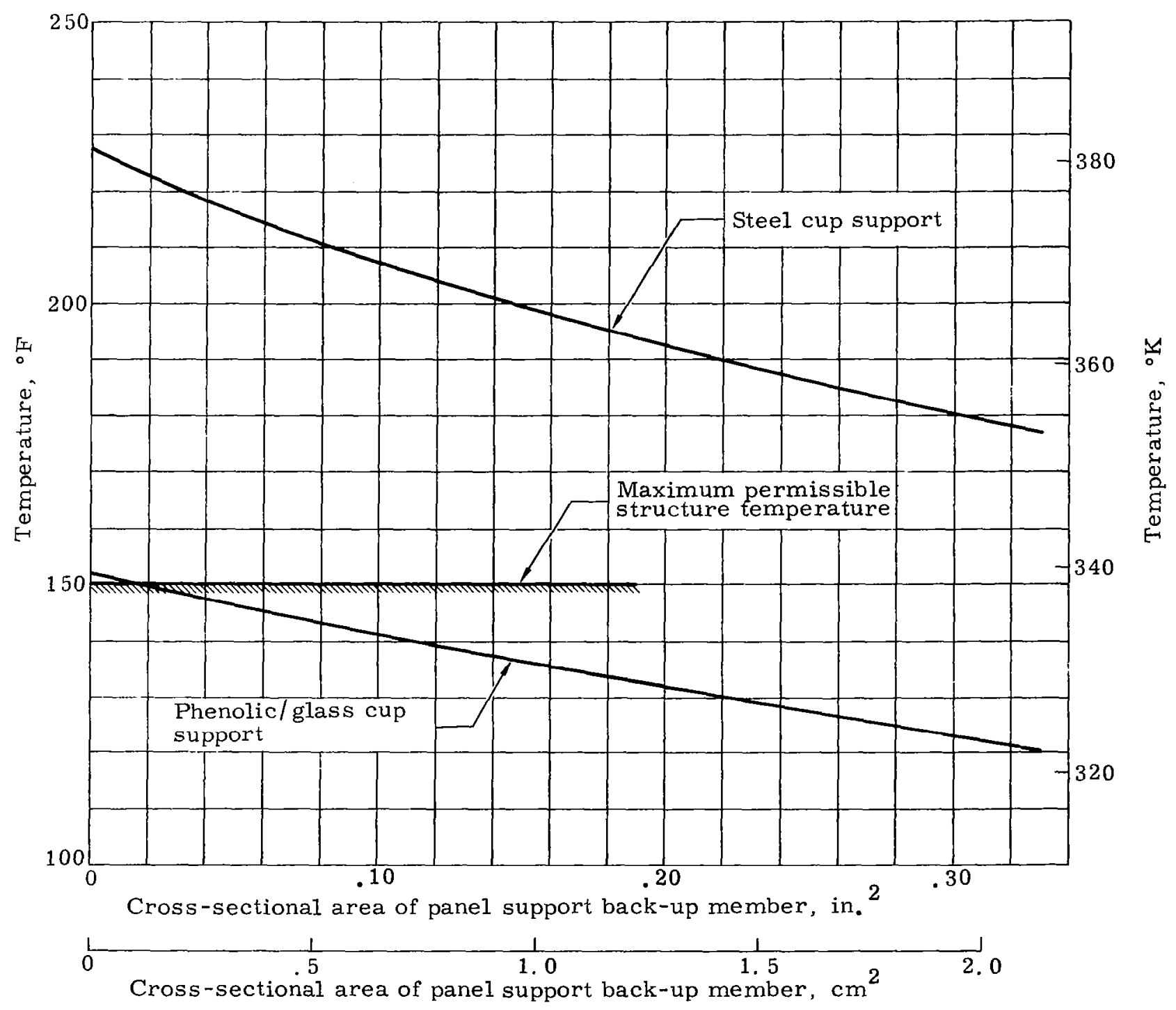

FIGURE 66. MAXIMUM STRUCTURE TEMPERATURE AT TOUCHDOWN WITH HEAT SHORT EFFECT CONSIDERED 


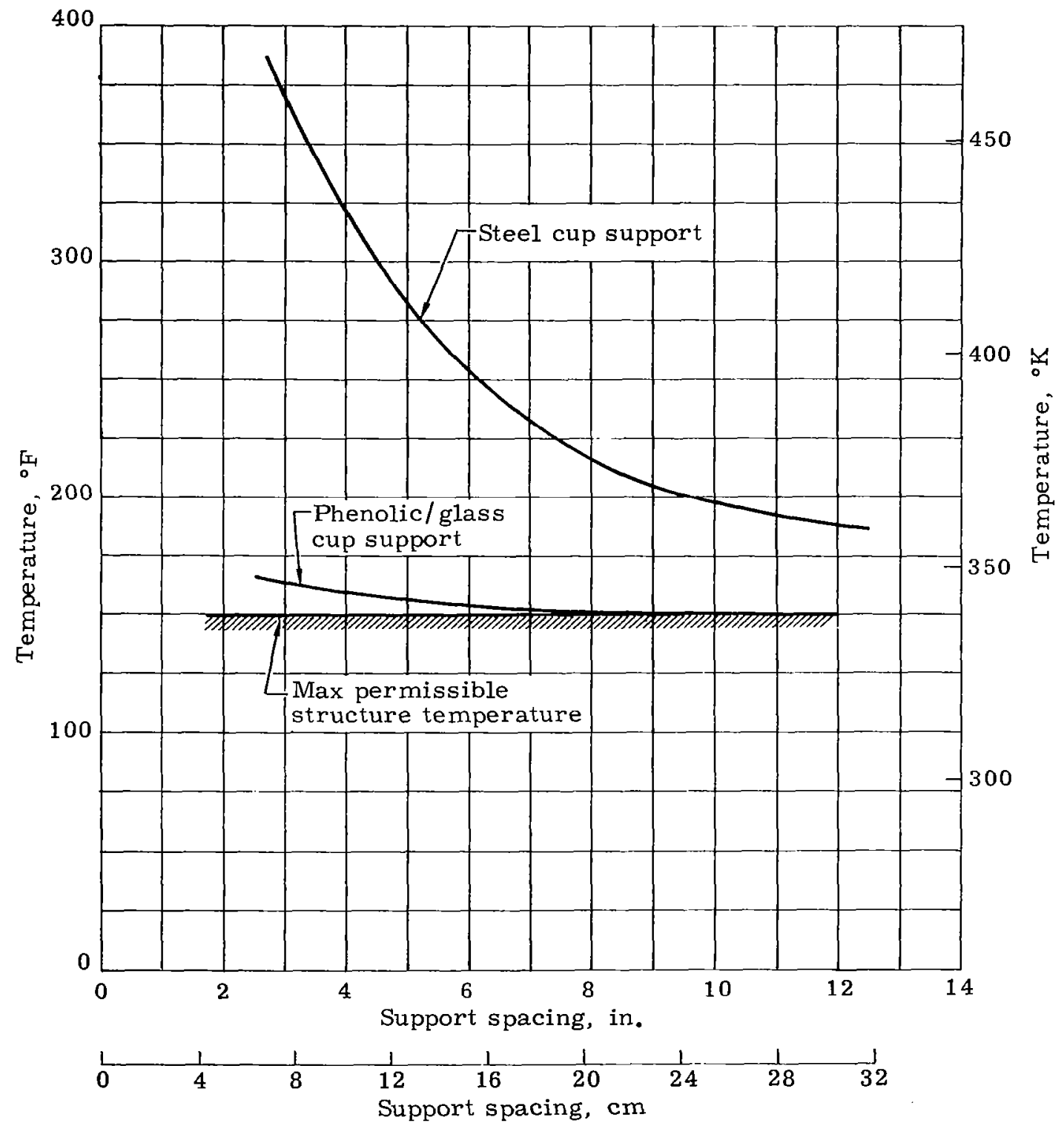

FIGURE 67. EFFECT OF SUPPORT SPACING ON HEAT SHORT EFFECT (ALUMINUM SKIN ONLY, NO HARD POINT) 
Moment

\begin{tabular}{|c|c|c|}
\hline $\begin{array}{l}\text { Curve } \\
\text { code }\end{array}$ & in.lb/in. & $\mathrm{cm}-\mathrm{kN} / \mathrm{cm}$ \\
\hline A & 2500 & 11.20 \\
B & 2000 & 8.96 \\
C & 1500 & 6.72 \\
D & 1000 & 4.48 \\
E & 800 & 3.58 \\
F & 600 & 2.68 \\
G & 400 & 1.79 \\
H & 200 & 0.89 \\
I & 100 & 0.44 \\
\hline
\end{tabular}

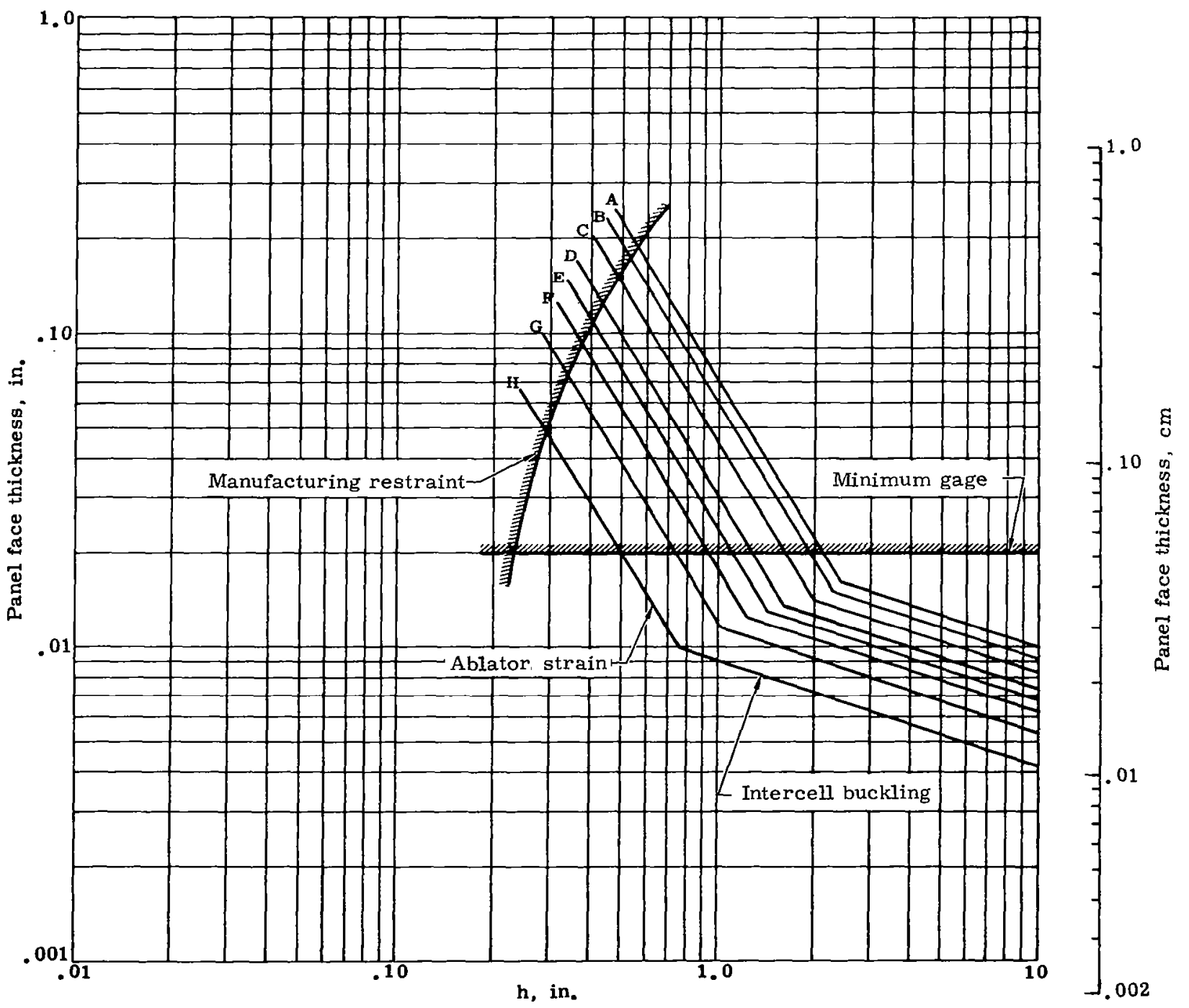

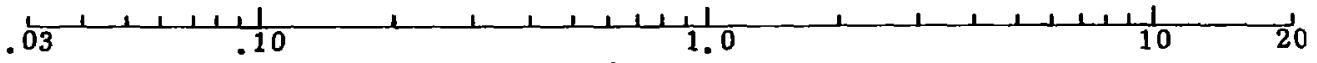

$$
\begin{aligned}
& h, \mathrm{~cm}
\end{aligned}
$$

FIGURE 68. SUBSTRATE PANEL DESIGN CONSTRAINTS [PHENOLIC/GLASS SUBSTRATE PANEL WITH $0.5-1 \mathrm{~N} .(1.27 \mathrm{CM})$ LDNP ABLATOR, $70^{\circ} \mathrm{F}\left(294^{\circ} \mathrm{K}\right)$ CONDITION] 


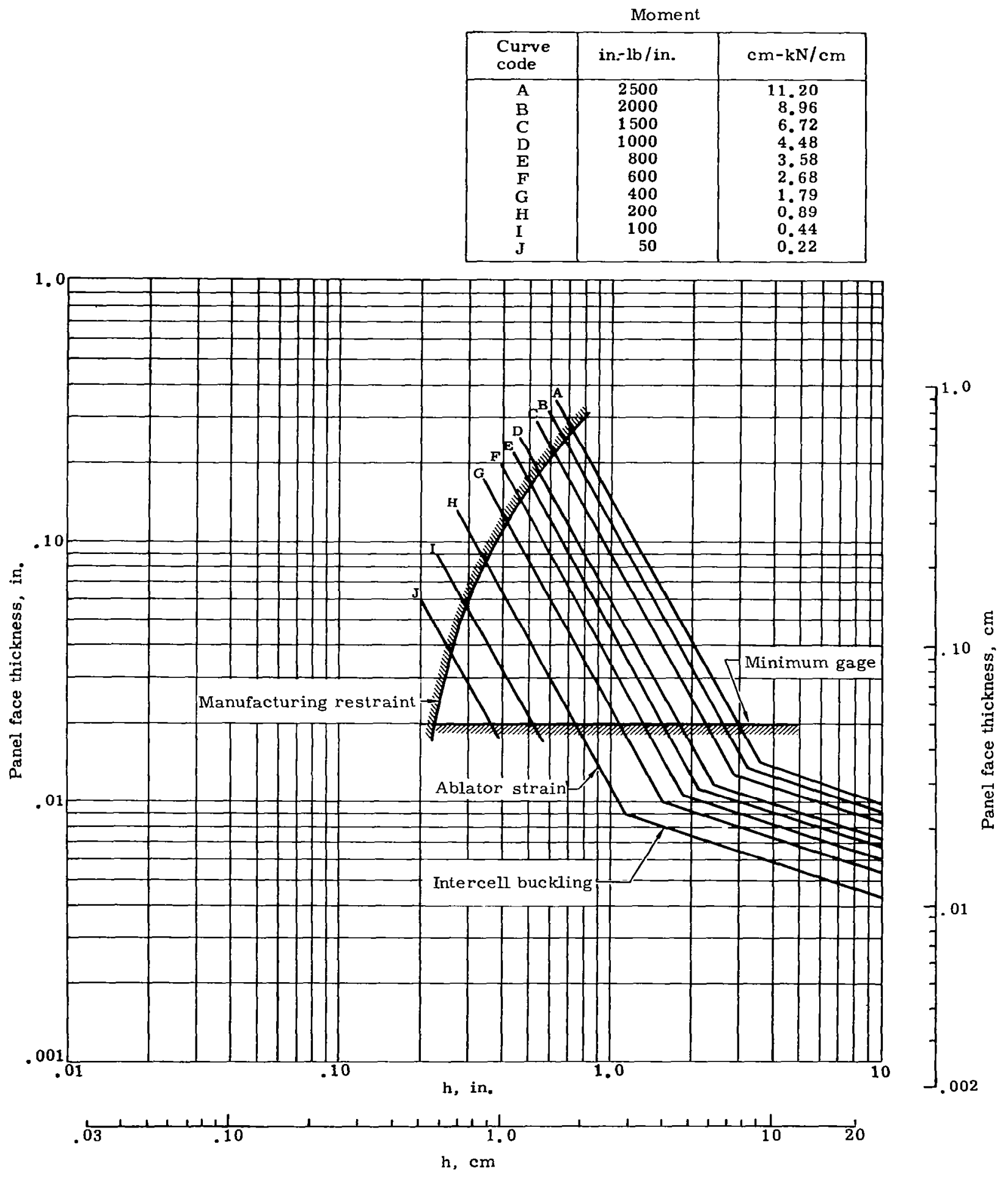

FIGURE 69. SUBSTRATE PANEL DESIGN CONSTRAINTS [PHENOLIC/GLASS SUBSTRATE PANEL WITH 1.50-IN. $(3.81 \mathrm{CM})$ LDNP ABLATOR, $70^{\circ} \mathrm{F}\left(294.4^{\circ} \mathrm{K}\right)$ CONDITION] 


\begin{tabular}{|c|c|c|}
\hline \multicolumn{3}{|c|}{ Moment } \\
$\begin{array}{l}\text { Curve } \\
\text { code }\end{array}$ & in.lb/in. & $\mathrm{cm}-\mathrm{kN} / \mathrm{cm}$ \\
\hline $\mathrm{A}$ & 2500 & 11.20 \\
$\mathrm{~B}$ & 2000 & 8.96 \\
$\mathrm{C}$ & 1500 & 6.72 \\
$\mathrm{D}$ & 1000 & 4.48 \\
$\mathrm{E}$ & 800 & 3.58 \\
$\mathrm{~F}$ & 600 & 2.68 \\
$\mathrm{G}$ & 400 & 1.79 \\
$\mathrm{H}$ & 200 & 0.89 \\
$\mathrm{I}$ & 100 & 0.44 \\
$\mathrm{~J}$ & 50 & 0.22 \\
\hline
\end{tabular}

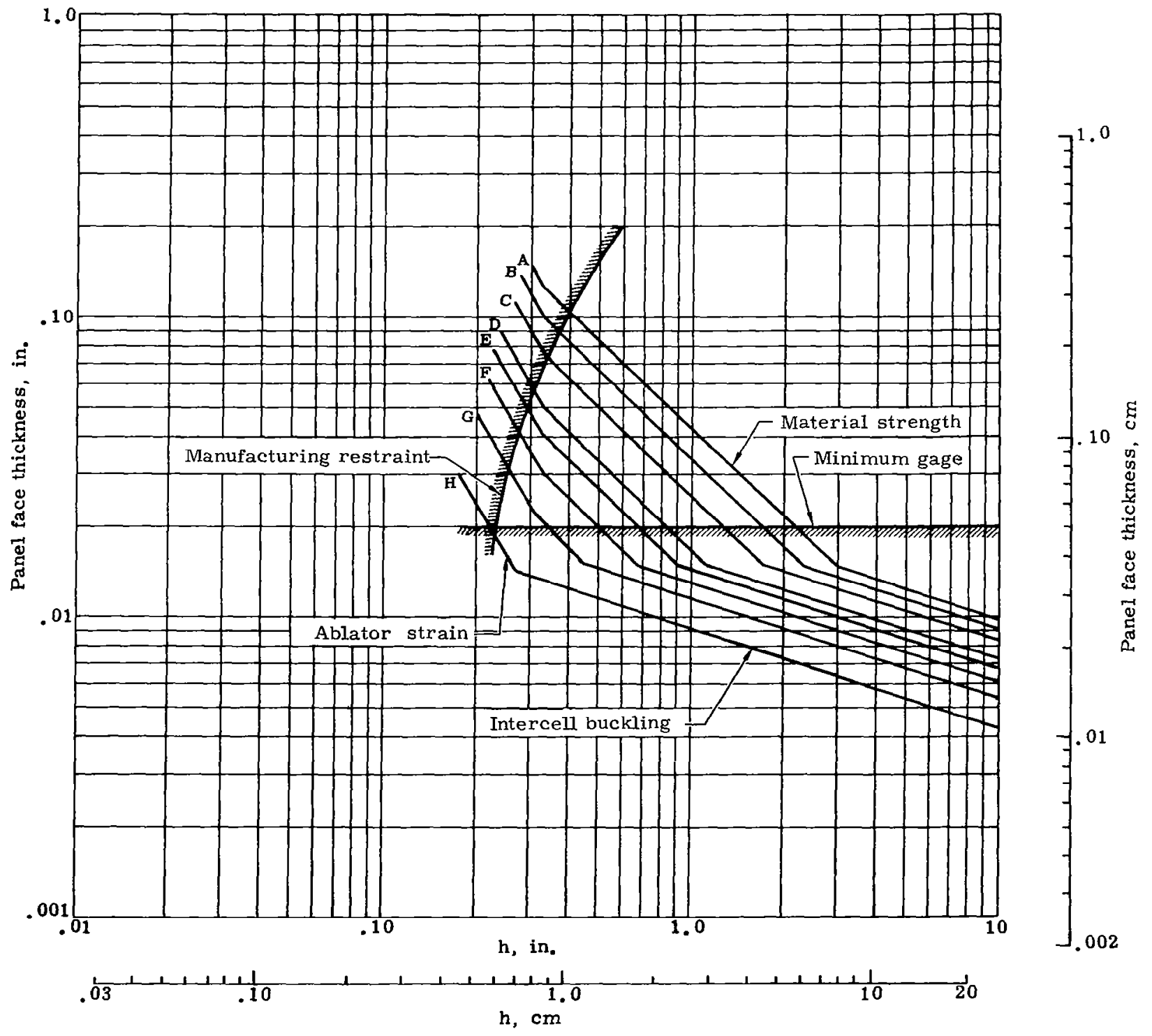

FIGURE 70. SUBSTRATE PANEL DESIGN CONSTRAINTS [PHENOLIC/GLASS SUBSTRATE PANEL WITH $0.50-1 \mathrm{~N} .(1.27 \mathrm{CM})$ NASA 602 ABLATOR, $70^{\circ} \mathrm{F}\left(294.4^{\circ} \mathrm{K}\right)$ CONDITION] 
Moment

\begin{tabular}{|c|c|c|}
\hline $\begin{array}{l}\text { Curve } \\
\text { code }\end{array}$ & in-lb/in. & $\mathrm{cm}-\mathrm{kN} / \mathrm{cm}$ \\
\hline A & 2500 & 11.20 \\
B & 2000 & 8.96 \\
C & 1500 & 6.72 \\
D & 1000 & 4.48 \\
E & 800 & 3.58 \\
F & 600 & 2.68 \\
G & 400 & 1.79 \\
H & 200 & 0.89 \\
I & 100 & 0.44 \\
J & 50 & 0.22 \\
\hline
\end{tabular}

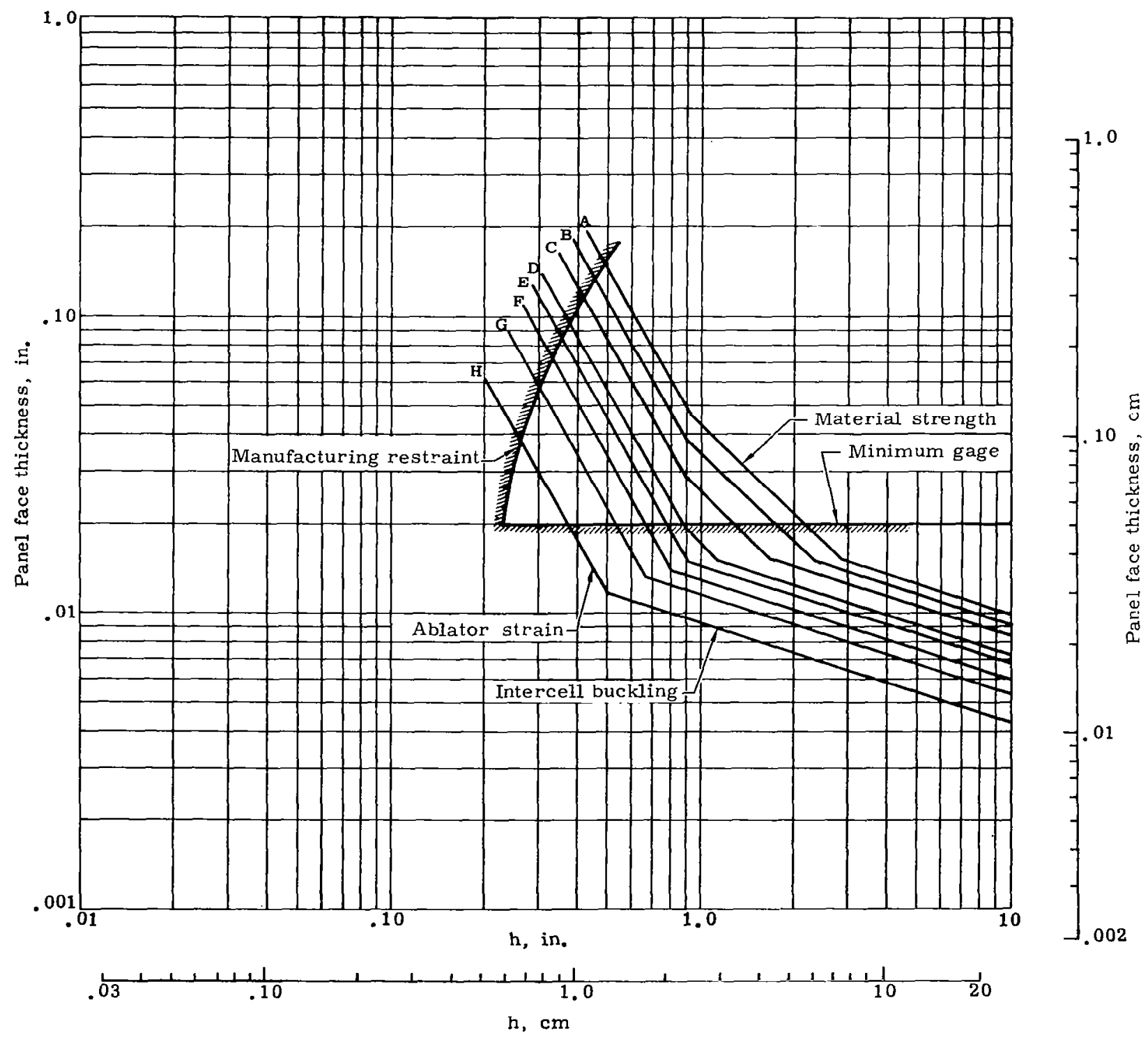

FIGURE 71. SUBGTRATE IANEL DESIGN CONSTRAINTS [PHENOLIC/GLASS SUBSTRATE PANEL WITH $1.50-1 \mathrm{~N}$. $(3.81 \mathrm{CM})$ NASA $602 \mathrm{ABLATOR}, 70^{\circ} \mathrm{F}\left(294.4^{\circ} \mathrm{K}\right)$ CONDITION] 

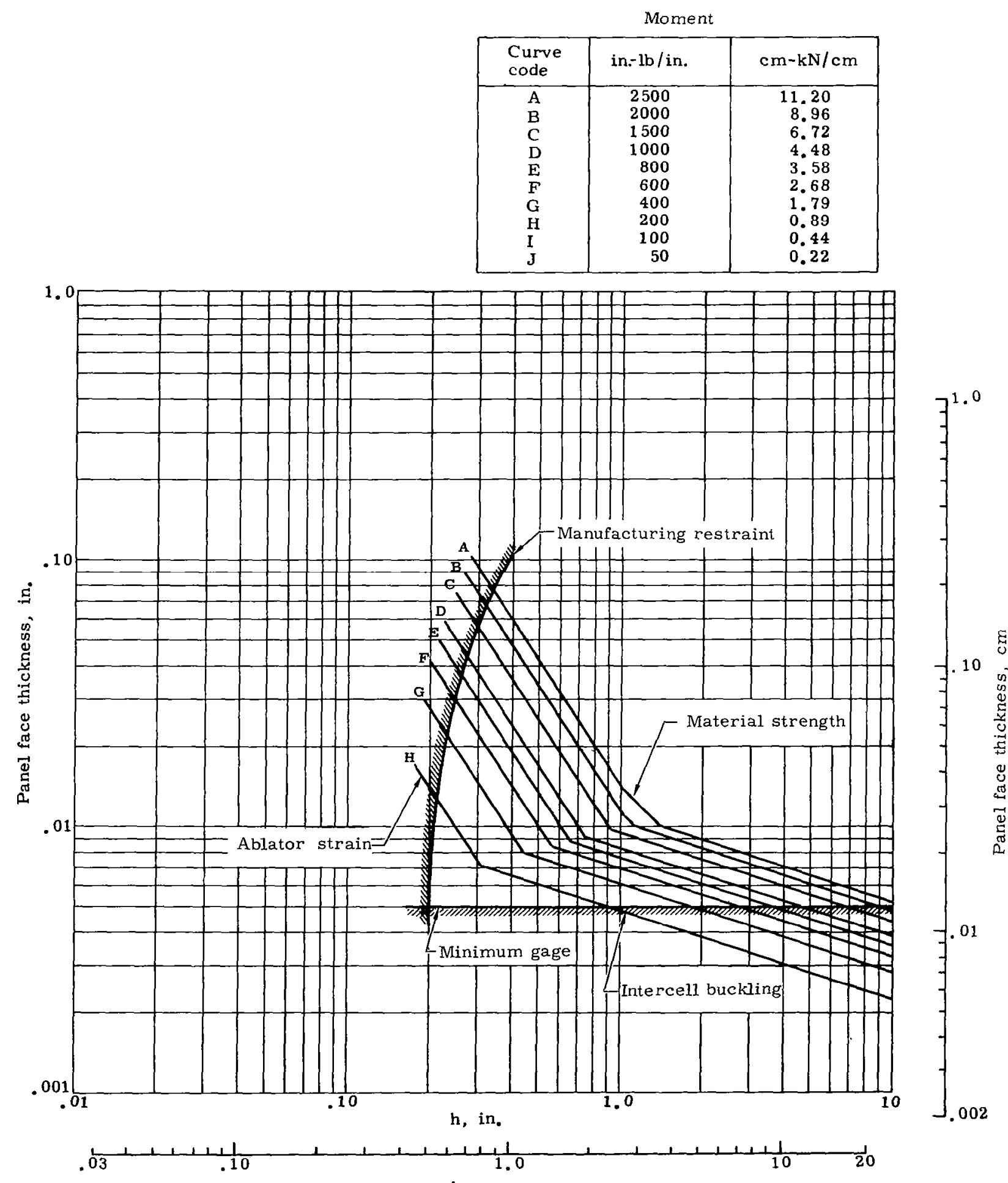

FIGURE 72. SUBSTRATE PANEL DESIGN CONSTRAINTS [17-7PH STEEL SUBSTRATE PANEL WITH $0.50-$ IN. (1.27 CM) LDNP ABLATOR, $70^{\circ} \mathrm{F}\left(294.4^{\circ} \mathrm{K}\right)$ CONDITION] 


\begin{tabular}{|c|c|c|}
\hline \multicolumn{3}{|c|}{ Moment } \\
$\begin{array}{c}\text { Curve } \\
\text { code }\end{array}$ & in-lb/in. & $\mathrm{cm}-\mathrm{kN} / \mathrm{cm}$ \\
\hline A & 2500 & 11.20 \\
B & 2000 & 8.96 \\
C & 1500 & 6.72 \\
D & 1000 & 4.48 \\
E & 800 & 3.58 \\
F & 600 & 2.68 \\
G & 400 & 1.79 \\
H & 200 & 0.89 \\
I & 100 & 0.44 \\
J & 50 & 0.22 \\
\hline
\end{tabular}

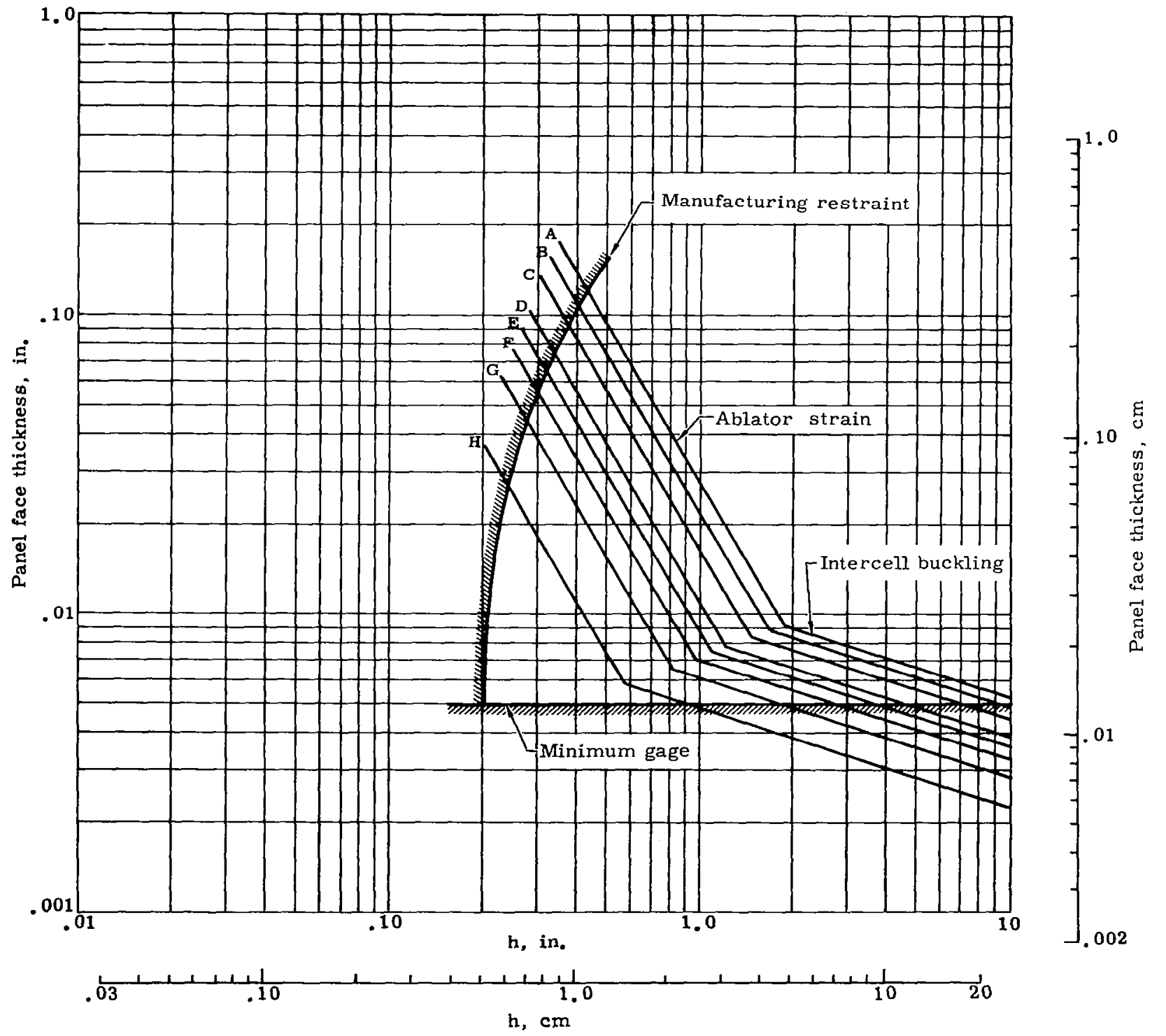

FIGURE 73. SUBSTRATE PANEL DESIGN CONSTRAINTS [17-7PH STEEL SUBSTRATE PANEL WITH $1.50-1 \mathrm{~N} .(3.81 \mathrm{CM})$ LDNP ABLATOR, $70^{\circ} \mathrm{F}\left(294.4^{\circ} \mathrm{K}\right)$ CONDITION] 


\begin{tabular}{|c|c|c|}
\hline \multicolumn{3}{|c}{ Moment } \\
\hline $\begin{array}{l}\text { Curve } \\
\text { code }\end{array}$ & in-lb/in. & $\mathrm{cm}-\mathrm{kN} / \mathrm{cm}$ \\
\hline A & 2500 & $\mathbf{1 1 . 2 0}$ \\
B & 2000 & 8.96 \\
C & 1500 & 6.72 \\
D & 1000 & 4.48 \\
F & 800 & 3.58 \\
G & 600 & 2.68 \\
H & 400 & 1.79 \\
I & 100 & 0.89 \\
J & 50 & 0.44 \\
\hline
\end{tabular}

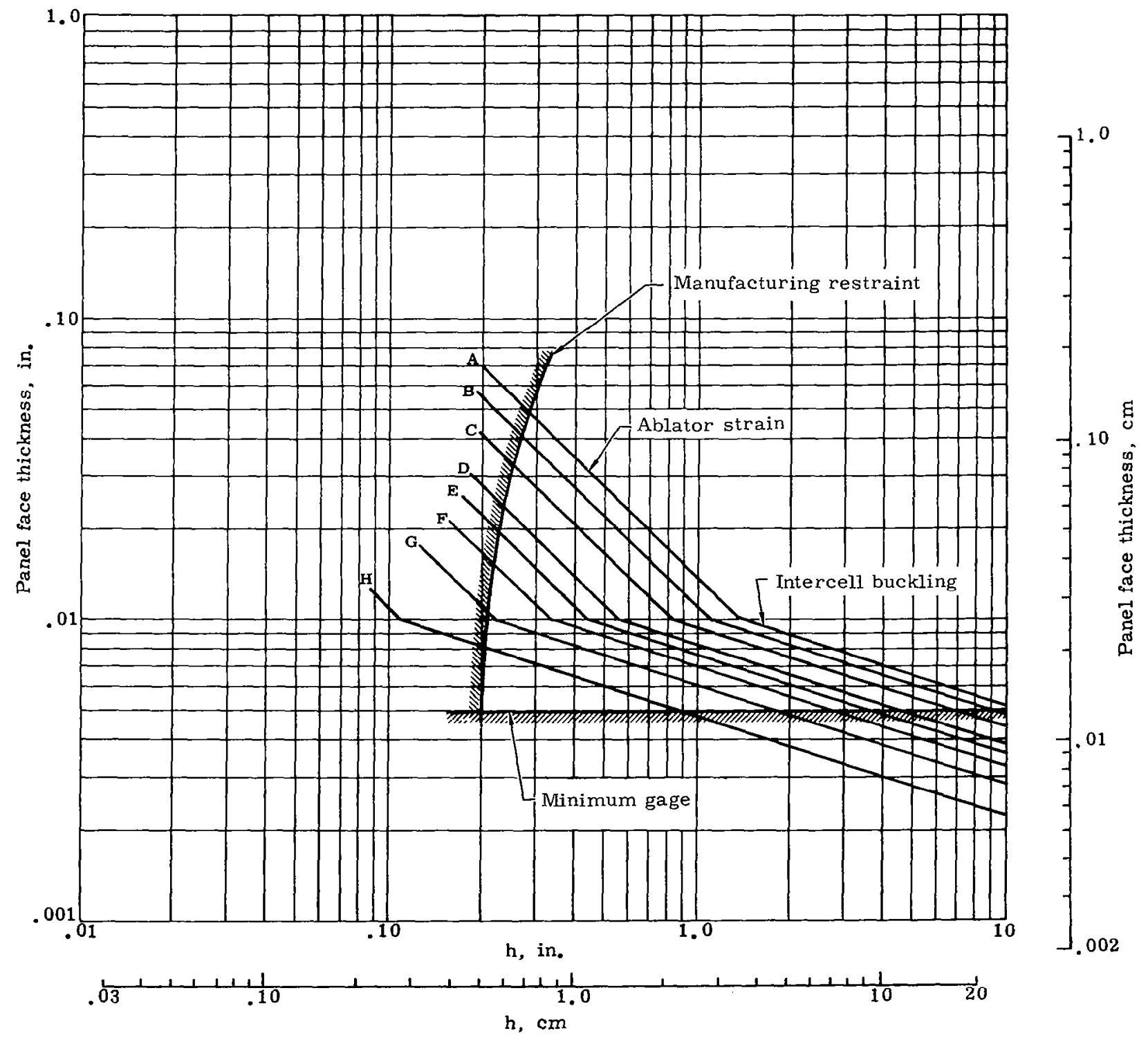

FIGURE 74. SUBSTRATE PANEL DESIGN CONSTRAINTS [17-7PH STEEL SUBSTRATE PANEL WITH $0.50-$ IN. ( $1.27 \mathrm{CM})$ NASA 602 ABLATOR, $70^{\circ} \mathrm{F}\left(294.4^{\circ} \mathrm{K}\right)$ CONDITION] 
Moment

\begin{tabular}{|c|c|c|}
\hline $\begin{array}{l}\text { Curve } \\
\text { code }\end{array}$ & in.lb/in. & $\mathrm{cm}-\mathrm{kN} / \mathrm{cm}$ \\
\hline A & 2500 & 11.20 \\
B & 2000 & 8.96 \\
C & 1500 & 6.72 \\
D & 1000 & 4.48 \\
E & 800 & 3.58 \\
F & 600 & 2.68 \\
G & 400 & 1.79 \\
H & 200 & 0.89 \\
I & 100 & 0.44 \\
J & 50 & 0.22 \\
\hline
\end{tabular}
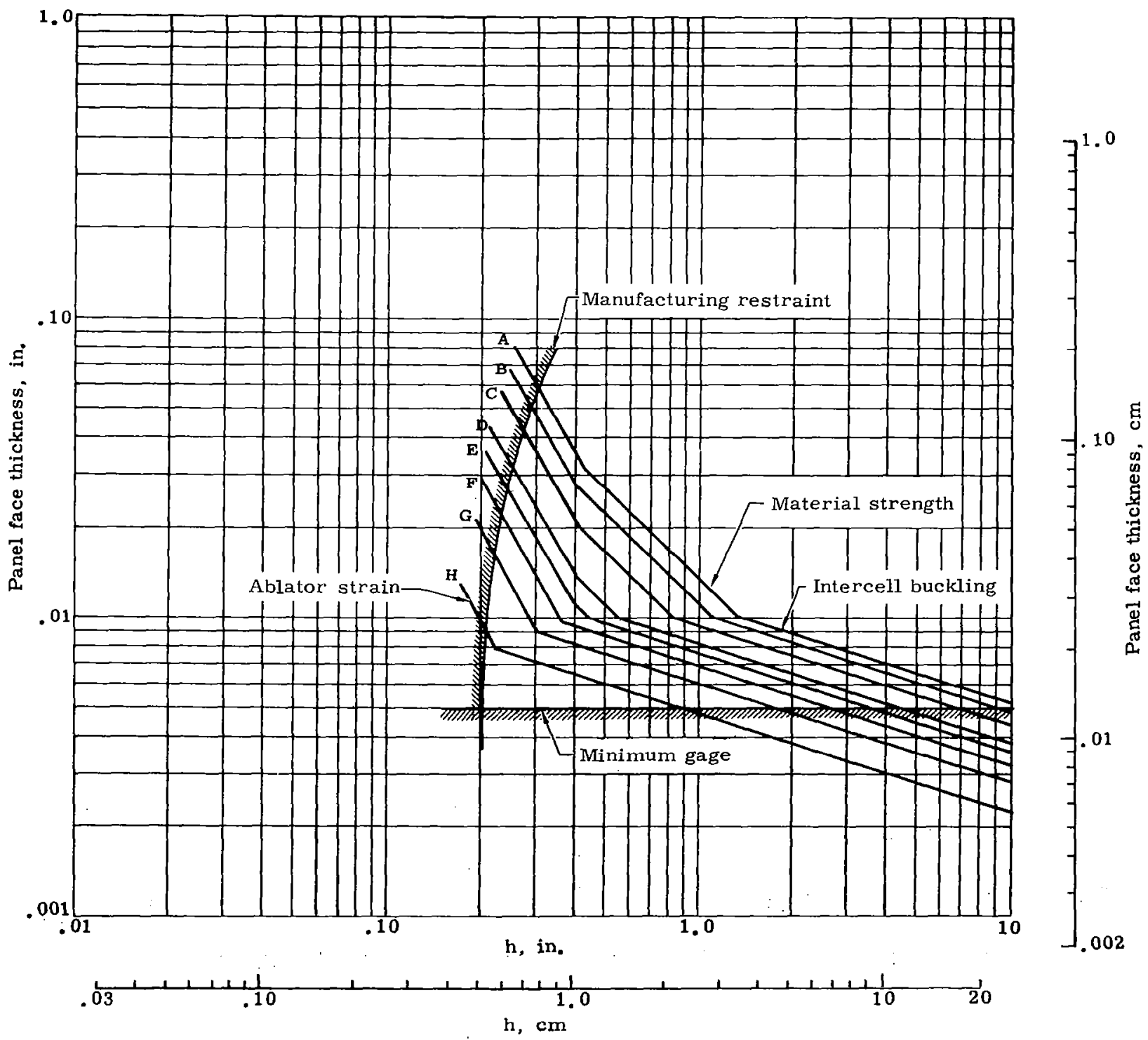

FIGURE 75. SUBSTRATE PANEL DESIGN CONSTRAINTS [17-7PH STEEL SUBSTRATE PANEL WITH $1.50-1 N .(3.81 \mathrm{CM})$ NASA 602 ABLATOR, $70^{\circ} \mathrm{F}\left(294.4^{\circ} \mathrm{K}\right)$ CONDITION] 


\begin{tabular}{|c|c|c|}
\hline \multicolumn{3}{|c|}{ Moment } \\
$\begin{array}{l}\text { Curve } \\
\text { code }\end{array}$ & in-lb/in. & $\mathrm{cm}-\mathrm{kN} / \mathrm{cm}$ \\
\hline A & 1000 & 4.48 \\
B & 800 & 3.58 \\
C & 600 & 2.68 \\
D & 400 & 1.79 \\
E & 200 & 0.89 \\
F & 100 & 0.44 \\
G & 50 & 0.22 \\
\hline
\end{tabular}
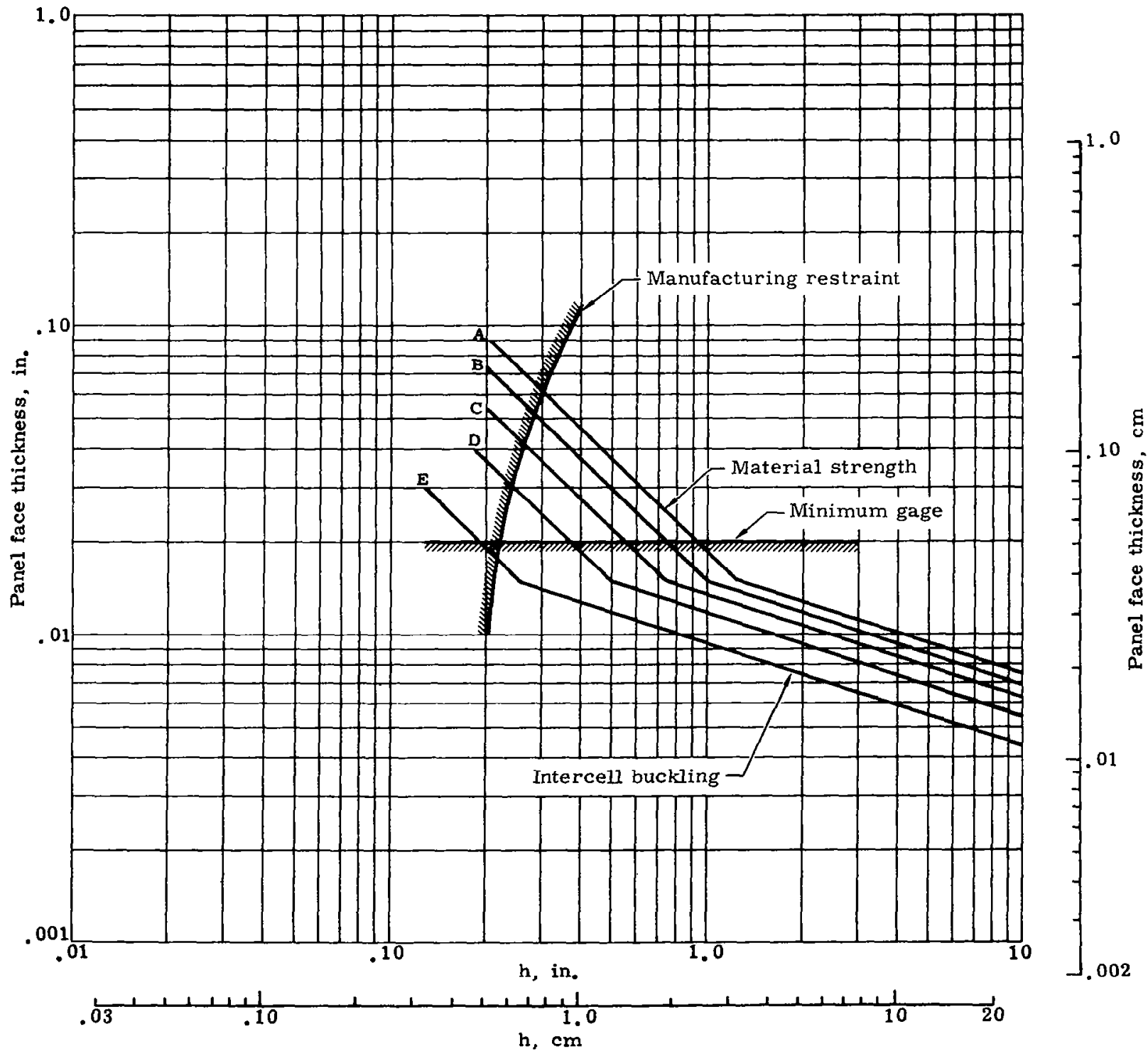

FIGURE 76. SUBSTRATE PANEL DESIGN CONSTRAINTS [PHENOLIC/GLASS SUBSTRATE PANEL, $300^{\circ} \mathrm{F}\left(422.2^{\circ} \mathrm{K}\right)$ CONDITION] 


\begin{tabular}{|c|c|c|}
\hline \multicolumn{3}{|c|}{ Moment } \\
$\begin{array}{c}\text { Curve } \\
\text { code }\end{array}$ & in- lb/in. & $\mathrm{cm}-\mathrm{kN} / \mathrm{cm}$ \\
\hline A & 1000 & 4.48 \\
B & 800 & 3.58 \\
C & 600 & 2.68 \\
D & 400 & 1.79 \\
E & 200 & 0.89 \\
F & 100 & 0.44 \\
G & 50 & 0.22 \\
\hline
\end{tabular}

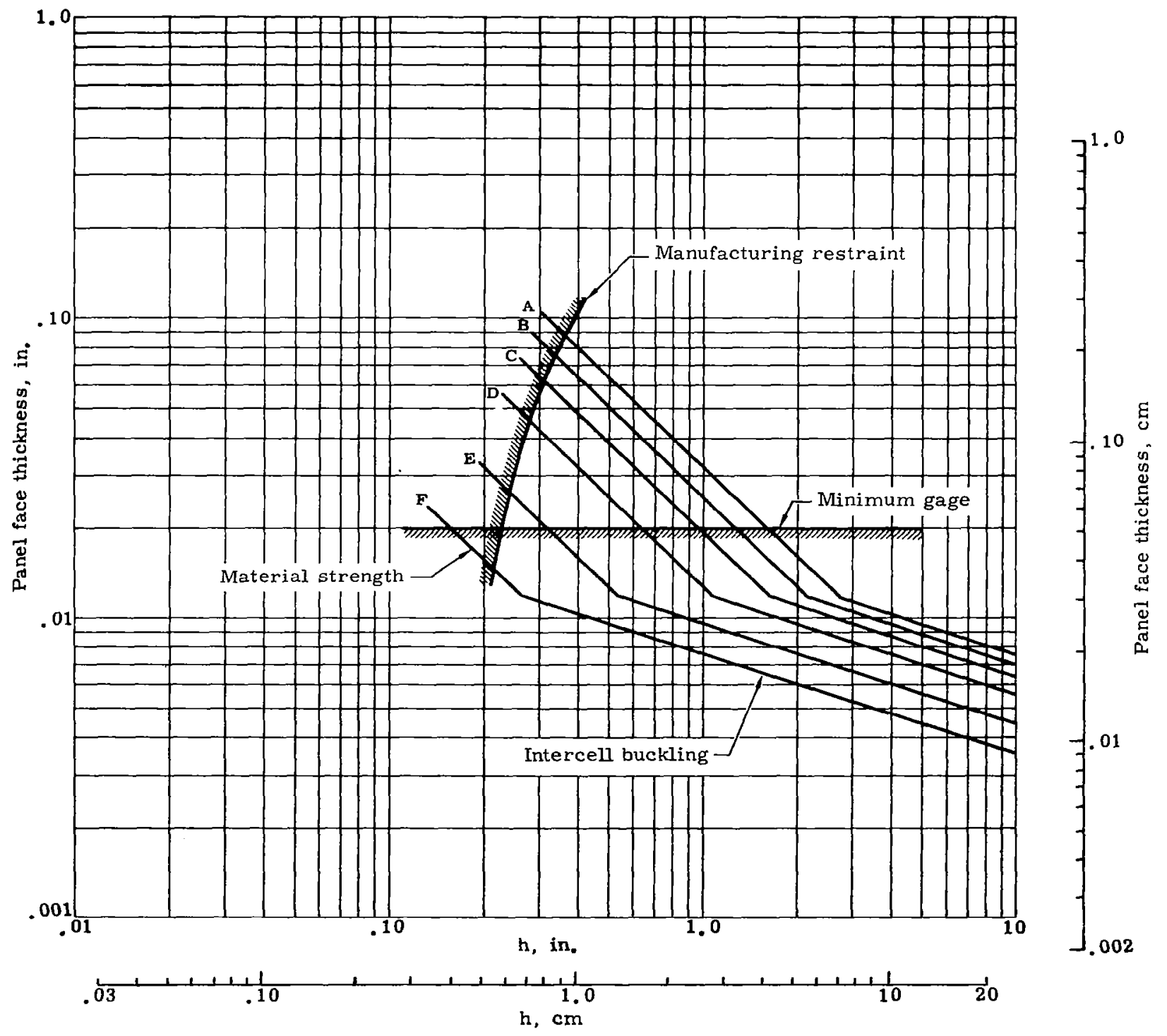

FIGURE 77. SUBSTRATE PANEL DESIGN CONSTRAINTS [PHENYL-SILANE/GLASS SIJBSTRATE PANEL, $300^{\circ} \mathrm{F}\left(422.2^{\circ} \mathrm{K}\right)$ CONDITION] 


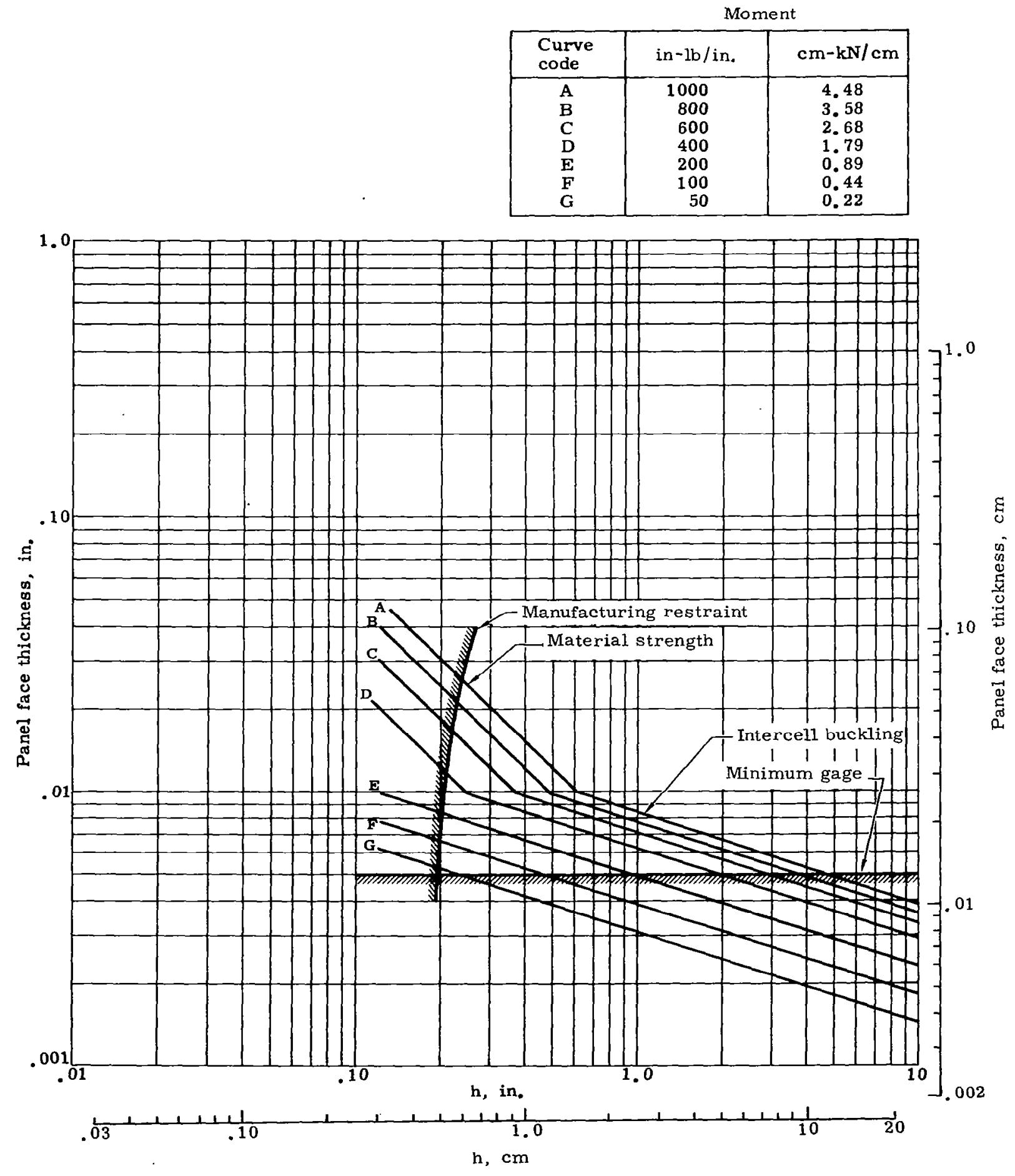

FIGURE 78. SUBSTRATE PANEL DESIGN CONSTRAINTS [17-7PH STEEL SUBSTRATE PANEL, $300^{\circ} \mathrm{F}\left(422.2^{\circ} \mathrm{K}\right)$ CONDITION] 
Moment

\begin{tabular}{|c|c|c|}
\hline $\begin{array}{l}\text { Curve } \\
\text { code }\end{array}$ & in.lb/in. & $\mathrm{cm}-\mathrm{kN} / \mathrm{cm}$ \\
\hline A & 1000 & 4.48 \\
B & 800 & 3.58 \\
C & 600 & 2.68 \\
D & 400 & 1.79 \\
E & 200 & 0.89 \\
F & 100 & 0.44 \\
\hline
\end{tabular}

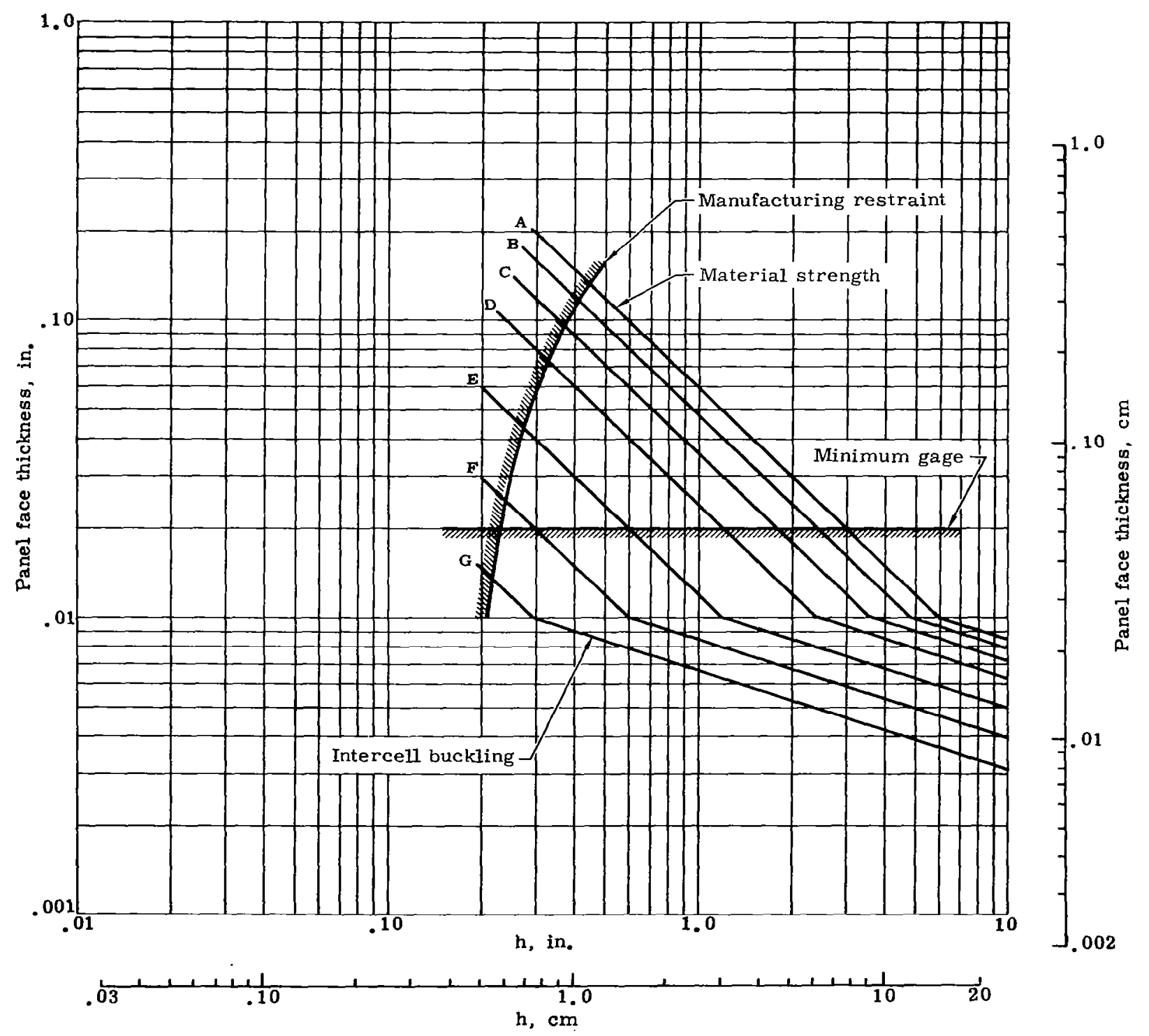

FIGURE 79. SUBSTRATE PANEL DESIGN CONSTRAINTS [PHENOLIC/GLASS SUBSTRATE PANEL, $800^{\circ} \mathrm{F}\left(700^{\circ} \mathrm{K}\right)$ CONDITION] 


\begin{tabular}{|c|c|c|}
\hline $\begin{array}{l}\text { Curve } \\
\text { code }\end{array}$ & in-lb/in. & $\mathrm{cm}-\mathrm{kN} / \mathrm{cm}$ \\
\hline $\begin{array}{l}\text { A } \\
\text { B } \\
C \\
D \\
\text { E } \\
\mathbf{F} \\
G\end{array}$ & $\begin{array}{r}1000 \\
800 \\
600 \\
400 \\
200 \\
100 \\
50\end{array}$ & $\begin{array}{l}4.48 \\
3.58 \\
2.68 \\
1.79 \\
0.89 \\
0.44 \\
0.22\end{array}$ \\
\hline
\end{tabular}

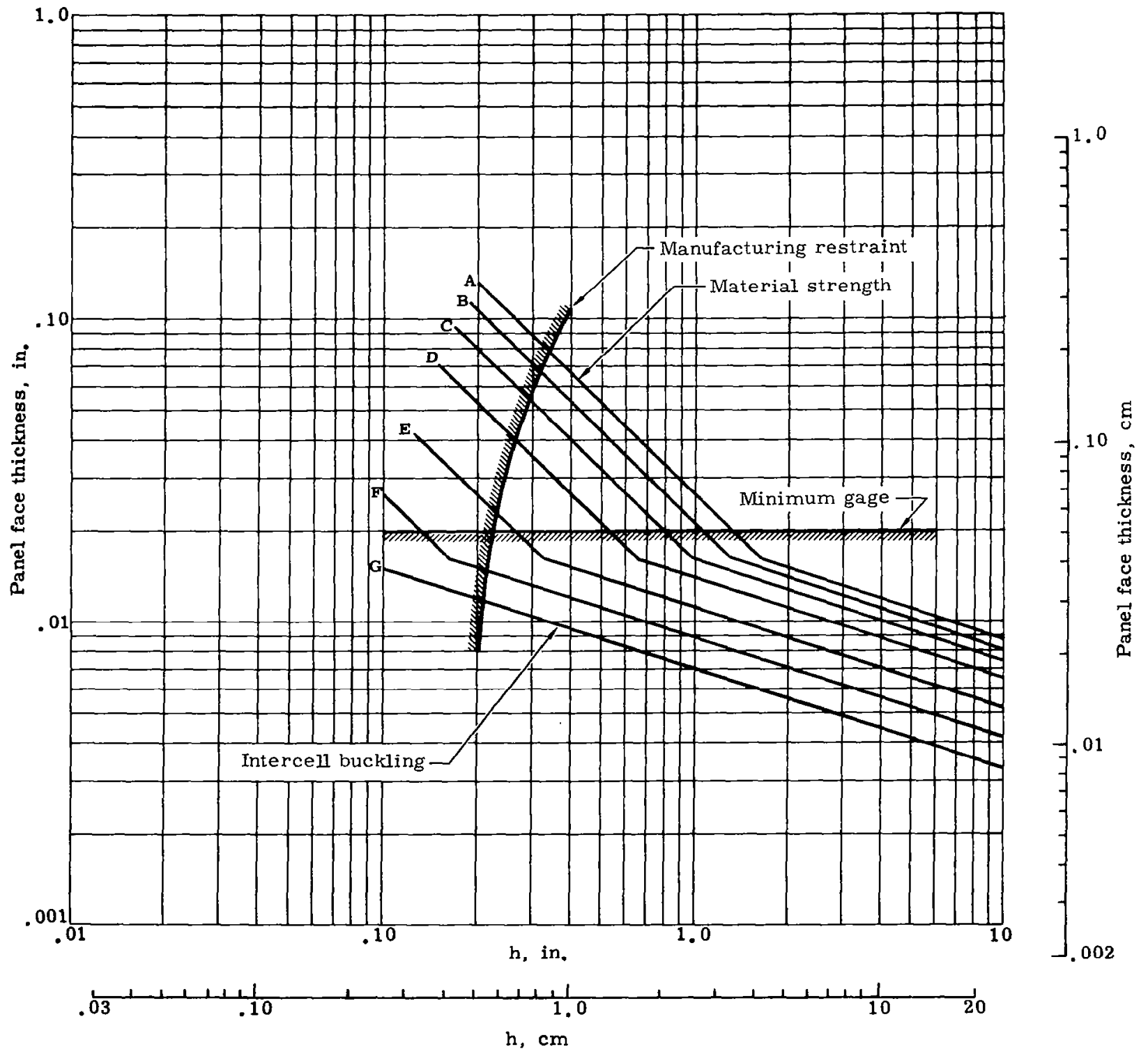

FIGURE 80. SUBSTRATE PANEL DESIGN CONSTRAINTS [PHENYL-SILANE/GLASS SUBSTRATE PANEL, $800^{\circ} \mathrm{F}\left(700^{\circ} \mathrm{K}\right)$ CONDITION] 
Moment

\begin{tabular}{|c|c|c|}
\hline $\begin{array}{l}\text { Curve } \\
\text { code }\end{array}$ & in-lb/in. & $\mathrm{cm}-\mathrm{kN} / \mathrm{cm}$ \\
\hline A & 1000 & 4.48 \\
B & 800 & 3.58 \\
C & 600 & 2.68 \\
D & 400 & 1.79 \\
E & 200 & 0.89 \\
F & 100 & 0.44 \\
G & 50 & 0.22 \\
\hline
\end{tabular}

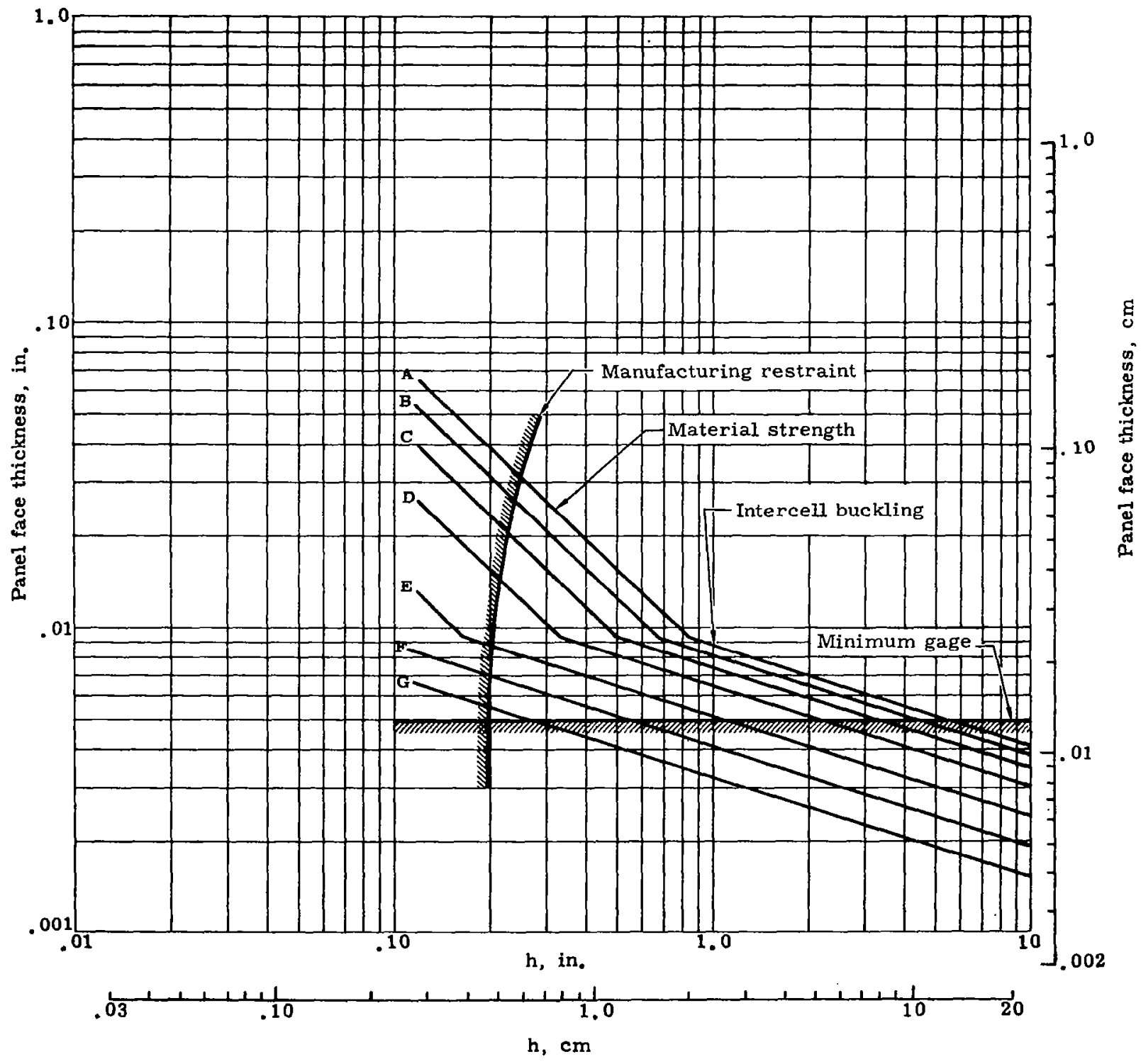

FIGURE 81. SUBSTRATE PANEL DESIGN CONSTRAINTS [17-7PH STEEL SUBSTRATE PANEL, $800^{\circ} \mathrm{F}\left(700^{\circ} \mathrm{K}\right)$ CONDITION] 


\begin{tabular}{|l|c|c|}
\hline \multicolumn{3}{|c|}{ Moment } \\
\hline $\begin{array}{l}\text { Curve } \\
\text { Code }\end{array}$ & in-lb/in. & $\mathrm{cm}-\mathrm{kN} / \mathrm{cm}$ \\
\hline A & 1000 &.--18 \\
B & 800 & 3.58 \\
C & 600 & 2.68 \\
D & 400 & 1.79 \\
E & 200 & 0.89 \\
F & 100 & 0.44 \\
G & 50 & 0.22 \\
\hline
\end{tabular}

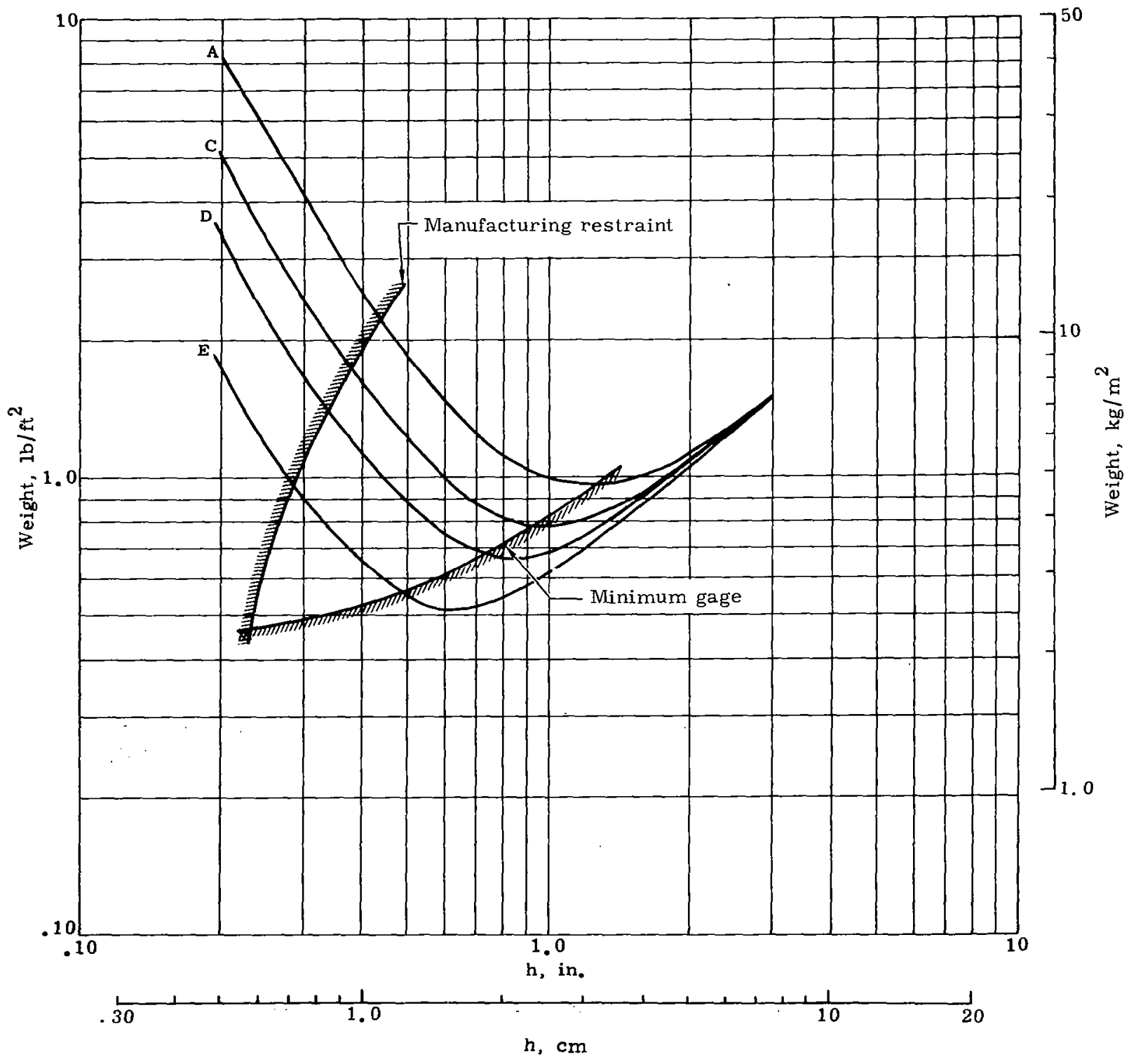

FIGURE 82. SUBSTRATE PANEL DESIGN OPTIMIZATION CURVES [PHENOLIC/GLASS SUBSTRATE WITH $0.50-$ IN. (1.27 CM) LDNP ABLATOR; $70^{\circ} \mathrm{F}\left(294.4^{\circ} \mathrm{K}\right)$ CONDITION] 
Moment

\begin{tabular}{|c|c|c|}
\hline $\begin{array}{l}\text { Curve } \\
\text { Code }\end{array}$ & in-1b/in. & $\mathrm{cm}-\mathrm{kN} / \mathrm{cm}$ \\
\hline A & 1000 & 4.48 \\
B & 800 & 3.58 \\
C & 600 & 2.68 \\
D & 400 & 1.79 \\
E & 200 & 0.89 \\
F & 100 & 0.44 \\
G & 50 & 0.22 \\
\hline
\end{tabular}

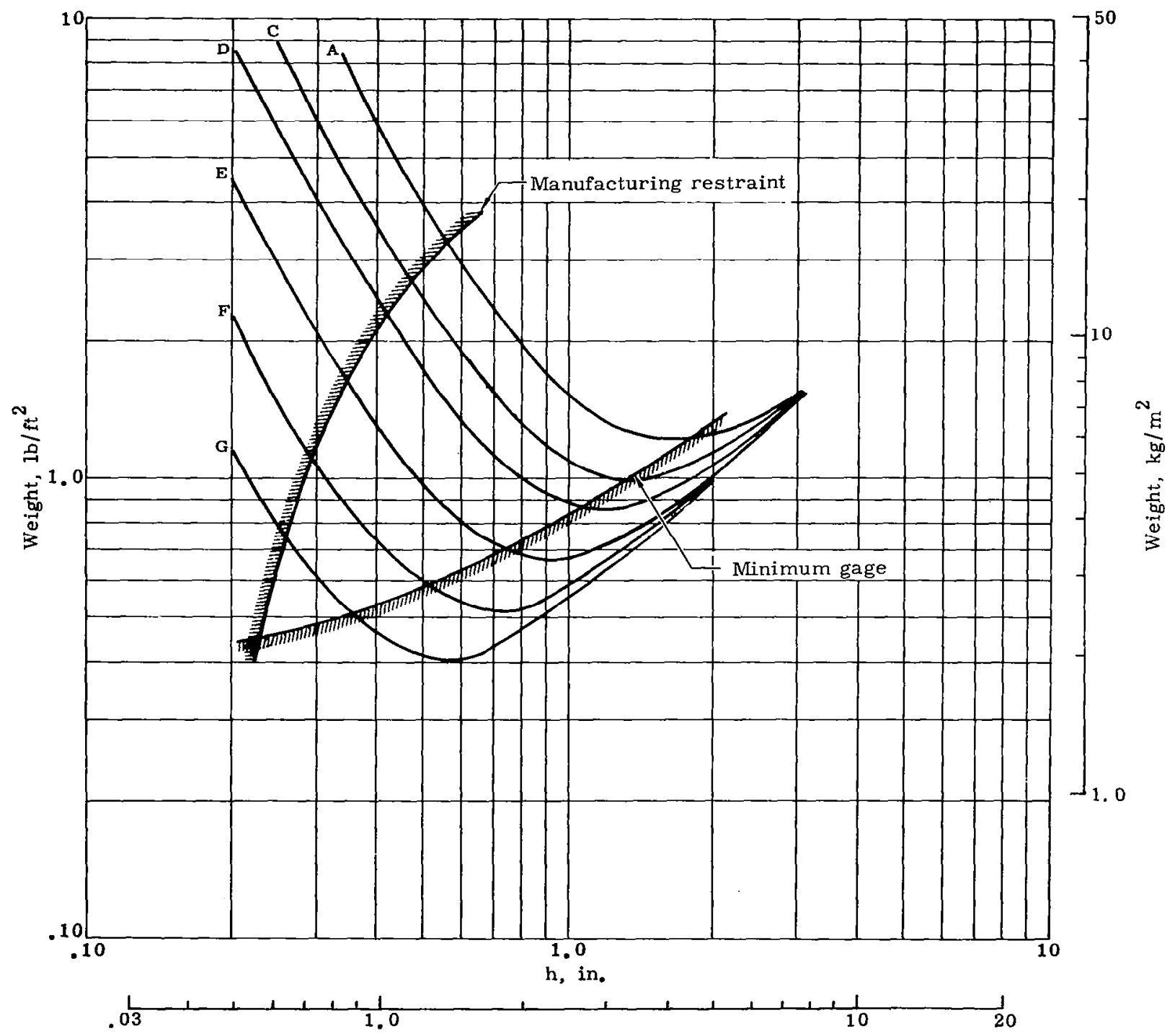

h, $\mathrm{cm}$

FIGIJRE 83. SUBSTRATE PANEL DESIGN OPTIMIZATION CURVES [PHENOLIC/GLASS SUBSTRATE WITH $1.50-1 \mathrm{~N} .(3.81 \mathrm{CM})$ LDNP ABLATOR, $70^{\circ} \mathrm{F}\left(294^{\circ} 4^{\circ} \mathrm{K}\right)$ CONDITION] 


\begin{tabular}{|l|c|c|}
\hline \multicolumn{2}{c|}{ Moment } \\
\hline $\begin{array}{l}\text { Curve } \\
\text { Code }\end{array}$ & in-lb/in. & $\mathrm{cm-kN/cm}$ \\
\hline A & 1000 & 4.48 \\
B & 800 & 3.58 \\
C & 600 & 2.68 \\
D & 400 & 1.79 \\
E & 200 & 0.89 \\
F & 100 & 0.44 \\
G & 50 & 0.22 \\
\hline
\end{tabular}

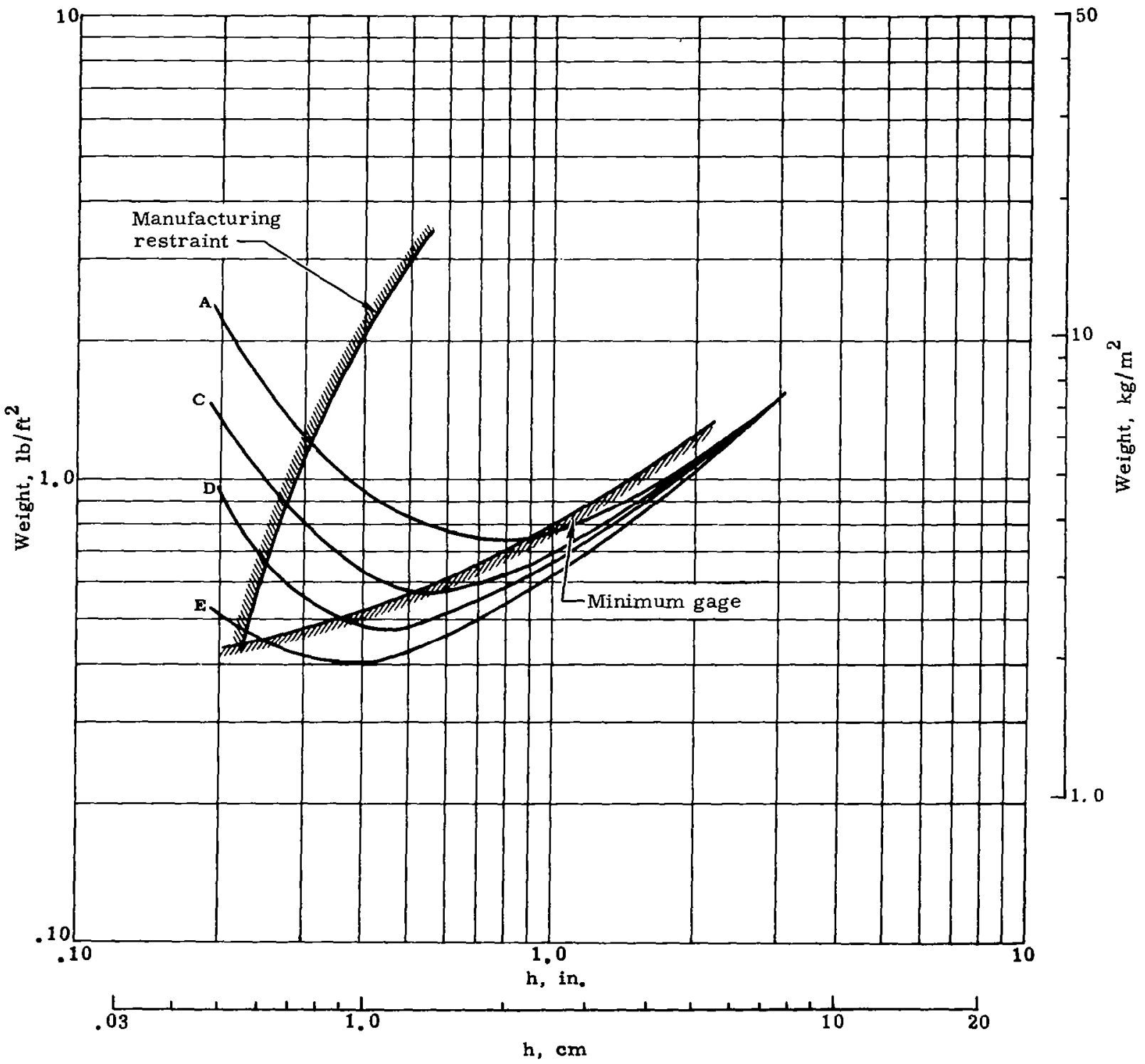

FIGURE 84. SUBSTRATE, PANEL DESIGN OPTIMIZATION CURVES [PHENOLIC/GLASS SUBSTRATE WITH $0.50-1 \mathrm{~N}$. (1.27 CM) NASA 602 ABLATOR, $70^{\circ} \mathrm{F}\left(294^{\circ} \mathrm{K}\right)$ CONDITION] 


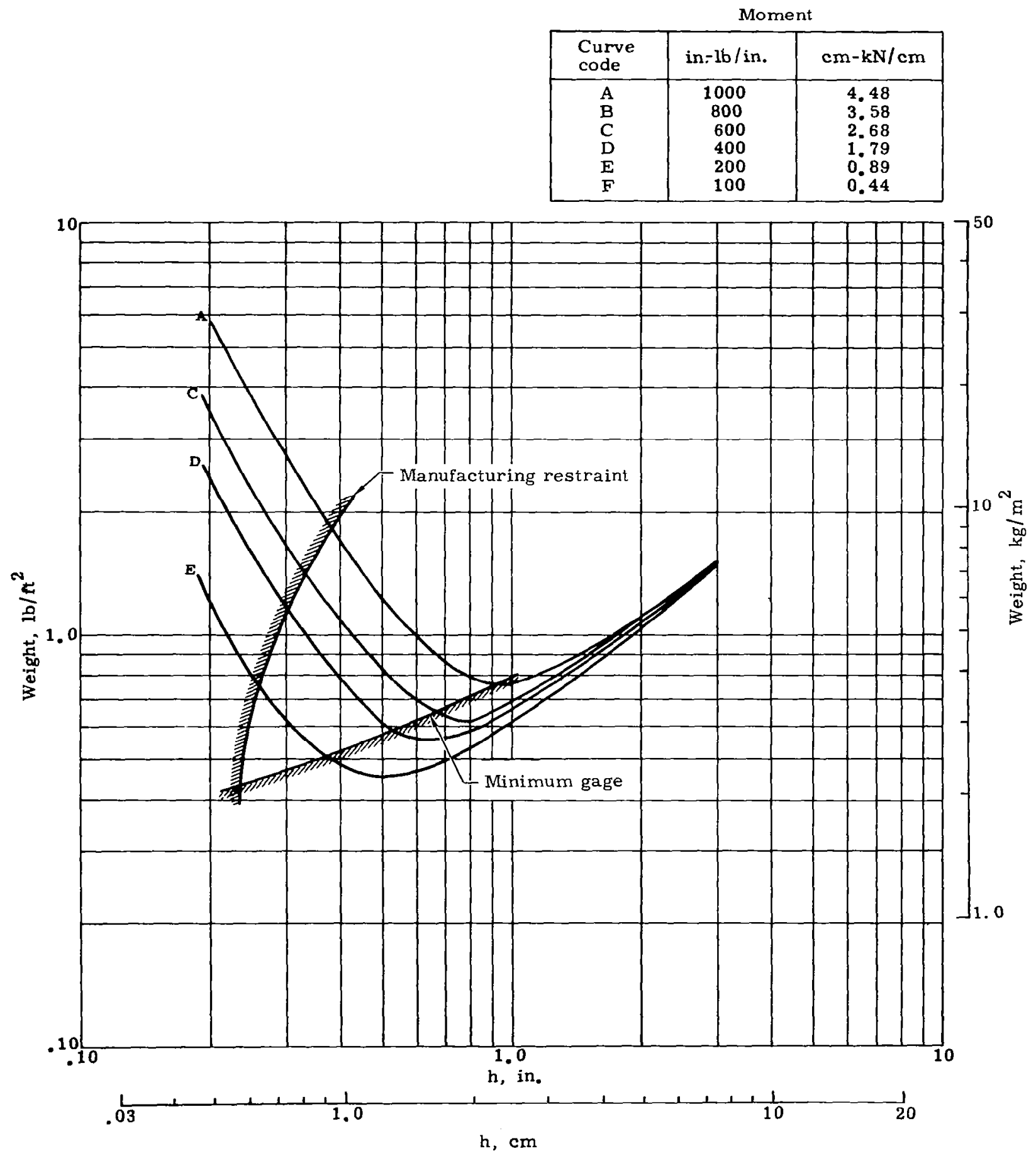

FIGURE 85. SUBSTRATE PANEL DESIGN OPTIMIZATION CURVES [PHENOLIC/GLASS SUBSTRATE WITH $1.50-I N .(3.81 \mathrm{CM})$ NASA 602 ABLATOR, $70^{\circ} \mathrm{F}$ $\left(294.4^{\circ} \mathrm{K}\right)$ CONDITION] 


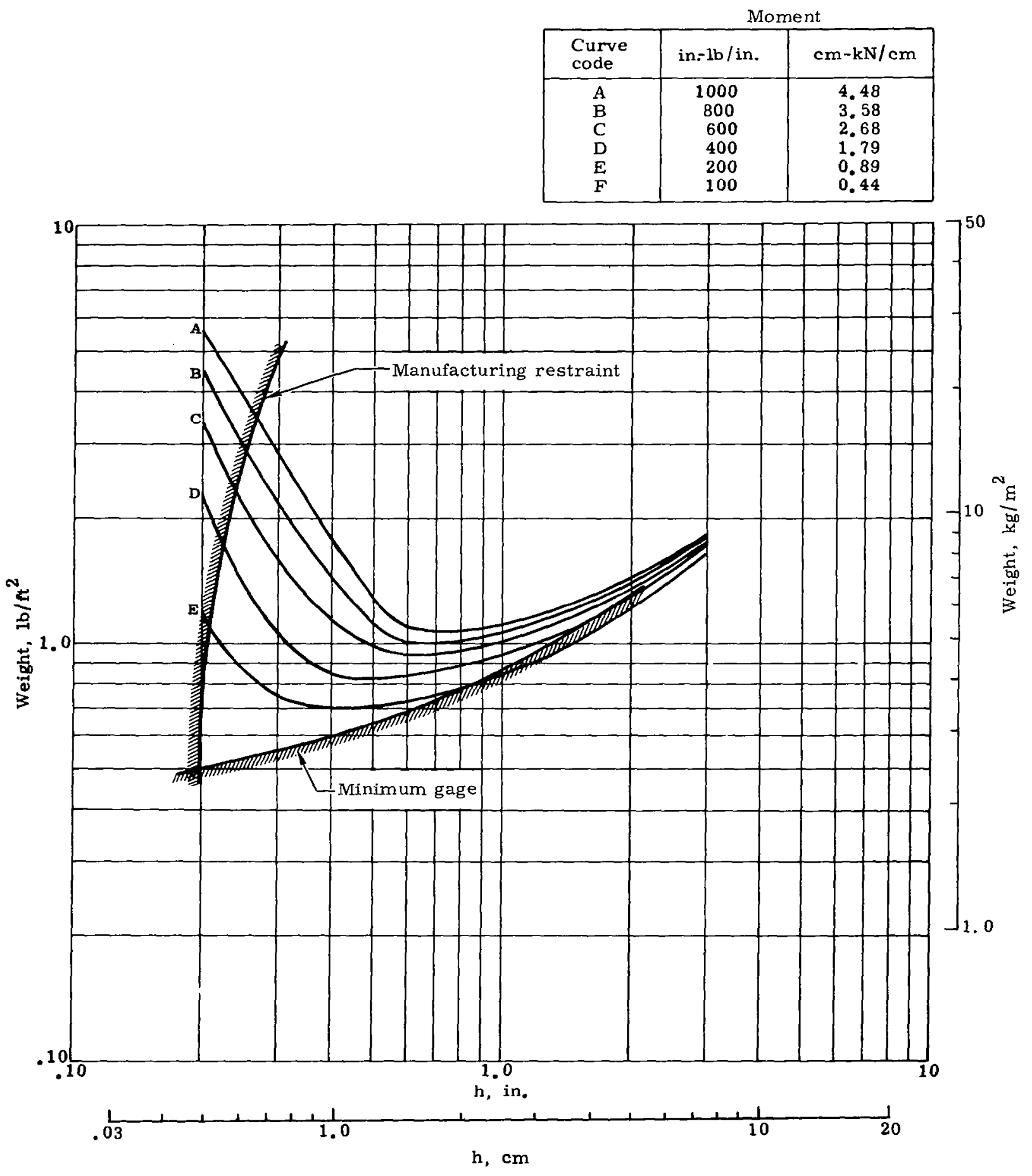

FIGURE 86. SUBSTRATE PANEL DESIGN OPTIMIZATION CURVES [17-7PH STEEL SUBSTRATE WITH $0.50-1 \mathrm{~N} .(1.27 \mathrm{CM})$ LDNP ABLATOR, $70^{\circ} \mathrm{F}\left(294.4^{\circ} \mathrm{K}\right)$ CONDITION] 


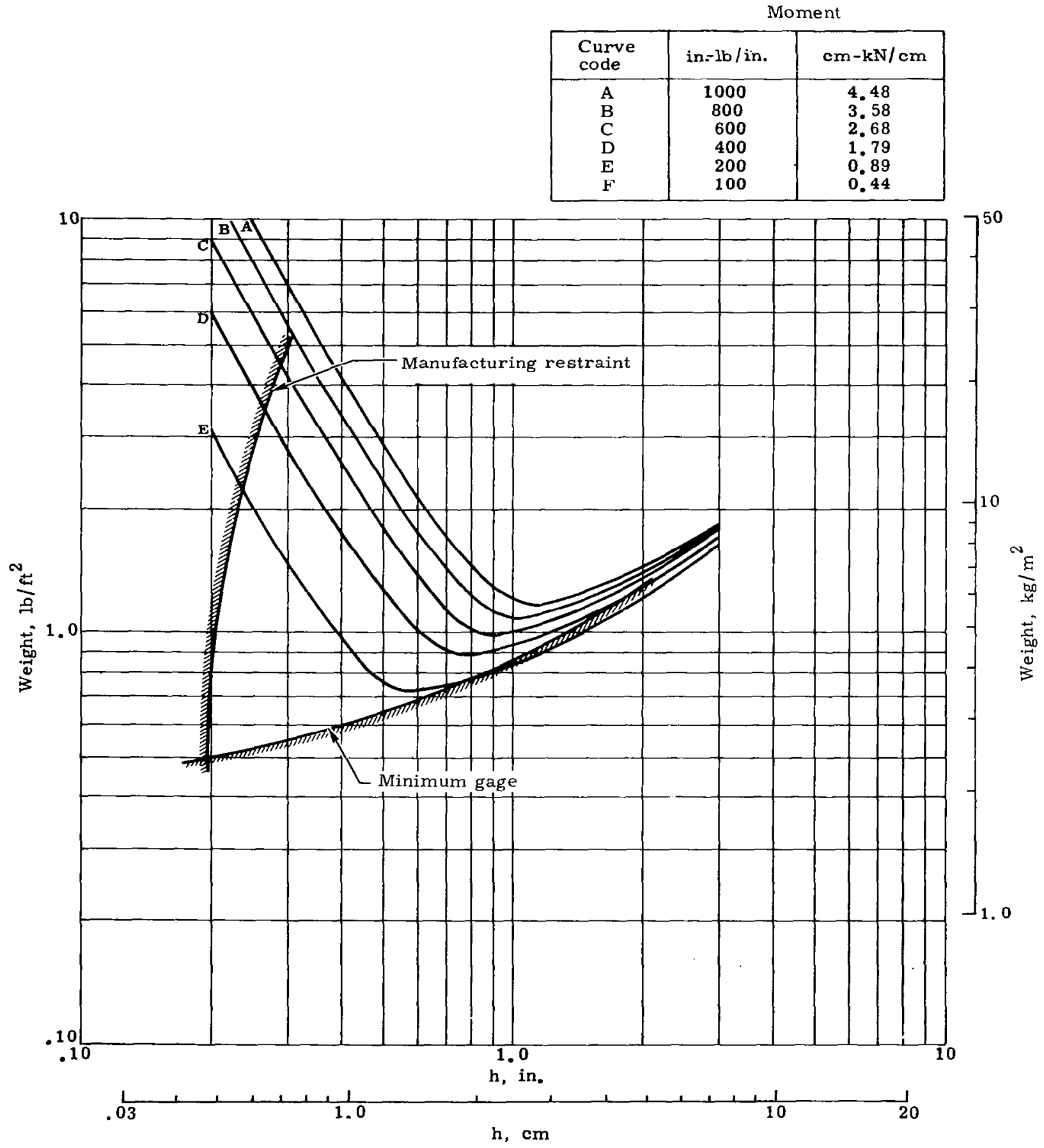

FIGURE 87. SUBSTRATE PANEL DESIGN OPTIMIZATION CURVES [ 17-7PH STEEL SUBSTRATE WITH $1.50-$ IN. $(3.81 \mathrm{CM})$ LDNP ABLATOR, $70^{\circ} \mathrm{F}\left(294^{\circ} \mathrm{K}\right)$ CONDITION] 


\begin{tabular}{|c|c|c|}
\hline \multicolumn{2}{|c|}{ Moment } \\
\hline $\begin{array}{l}\text { Curve } \\
\text { code }\end{array}$ & in-lb/in. & $\mathrm{cm}-\mathrm{kN} / \mathrm{cm}$ \\
\hline A & 1000 & 4.48 \\
B & 800 & 3.58 \\
C & 600 & 2.68 \\
D & 400 & 1.79 \\
E & 200 & 0.89 \\
F & 100 & 0.44 \\
\hline
\end{tabular}

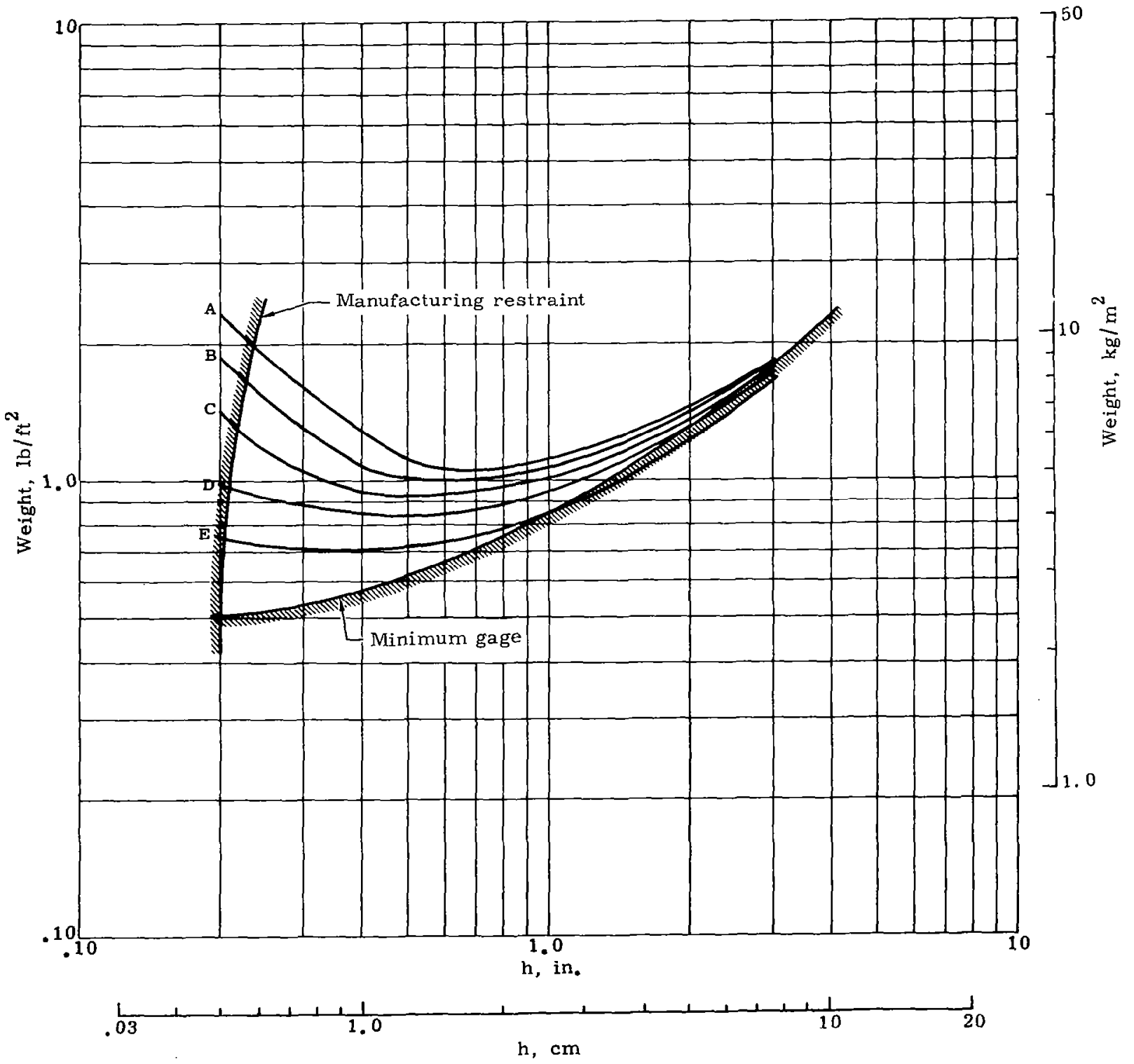

FIGURE 88. SUBSTRATE PANEL DESIGN OPTIMIZATION CURVES [17-7PH STEEL SUBSTRATE
WITH $0.50-1 \mathrm{~N} .(1.27 \mathrm{CM})$ NASA 602 ABLATOR, $70^{\circ} \mathrm{F}\left(294^{\circ} \mathrm{K}\right)$ CONDITION] 


\begin{tabular}{|l|c|c|}
\hline \multicolumn{3}{|c|}{ Moment } \\
\hline $\begin{array}{l}\text { Curve } \\
\text { Code }\end{array}$ & in-lb/in. & $\mathrm{cm}-\mathrm{kN} / \mathrm{cm}$ \\
\hline A & 1000 & 4.48 \\
B & 800 & 3.58 \\
C & 600 & 2.68 \\
D & 400 & 1.79 \\
E & 200 & 0.89 \\
F & 100 & 0.44 \\
G & 50 & 0.22 \\
\hline
\end{tabular}

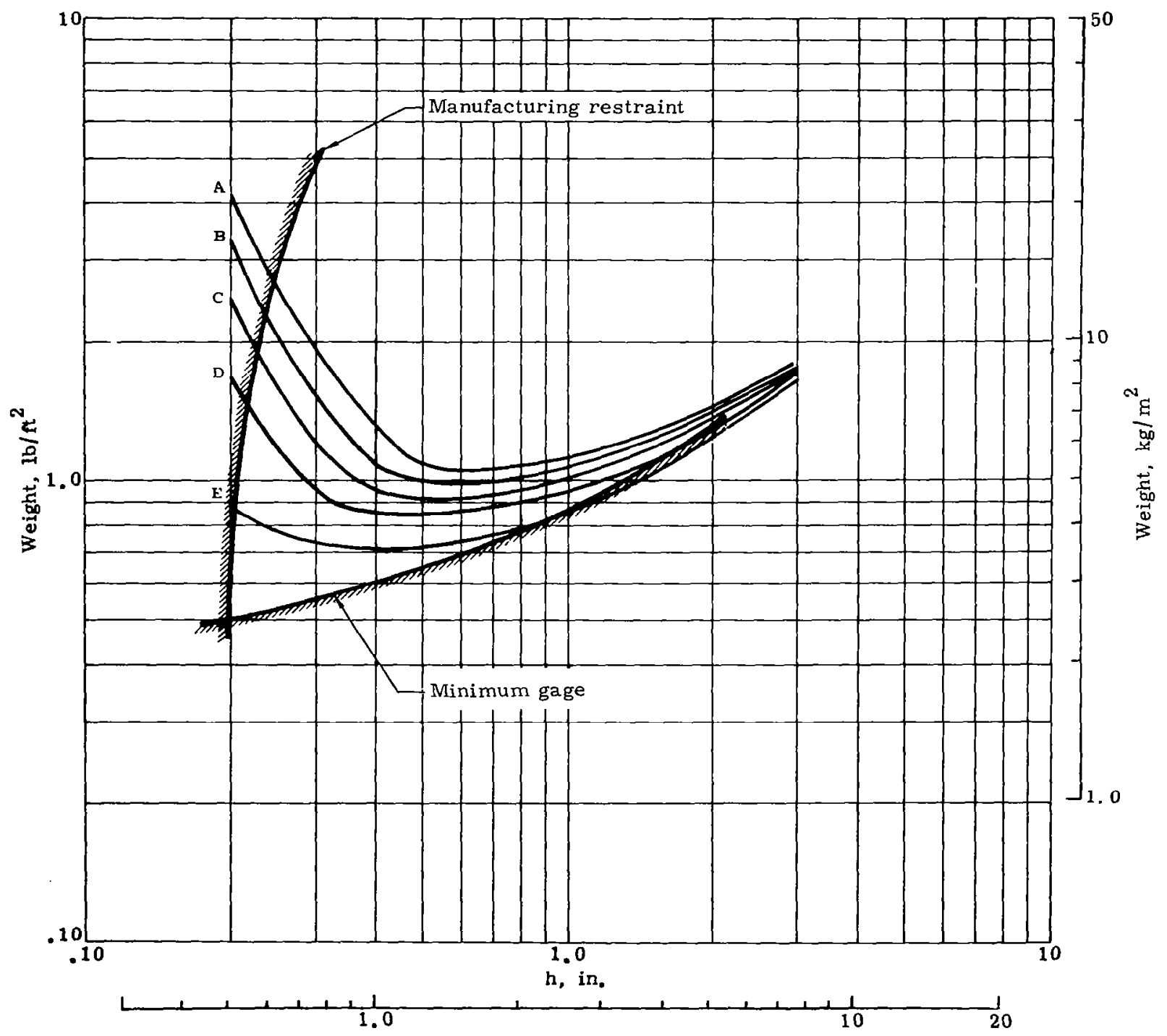

$\mathrm{h}, \mathrm{cm}$

FIGURE 89. SUBSTRATE PANEL DESIGN OPTIMIZATION CURVES [17-7PH STEEL SUBSTRATE WITH 1.50 -IN. $(3.81 \mathrm{CM})$ NASA 602 ABLATOR, $70^{\circ} \mathrm{F}\left(294.4^{\circ} \mathrm{K}\right)$ CONDITION] 


\begin{tabular}{|l|c|c|}
\hline \multicolumn{3}{|c|}{ Moment } \\
\hline $\begin{array}{l}\text { Curve } \\
\text { Code }\end{array}$ & in-lb/in. & $\mathrm{cm}-\mathrm{kN} / \mathrm{cm}$ \\
\hline A & 1000 & 4.48 \\
B & 800 & 3.58 \\
C & 600 & 2.68 \\
D & 400 & 1.79 \\
E & 200 & 0.89 \\
F & 100 & 0.44 \\
G & 50 & 0.22 \\
\hline
\end{tabular}

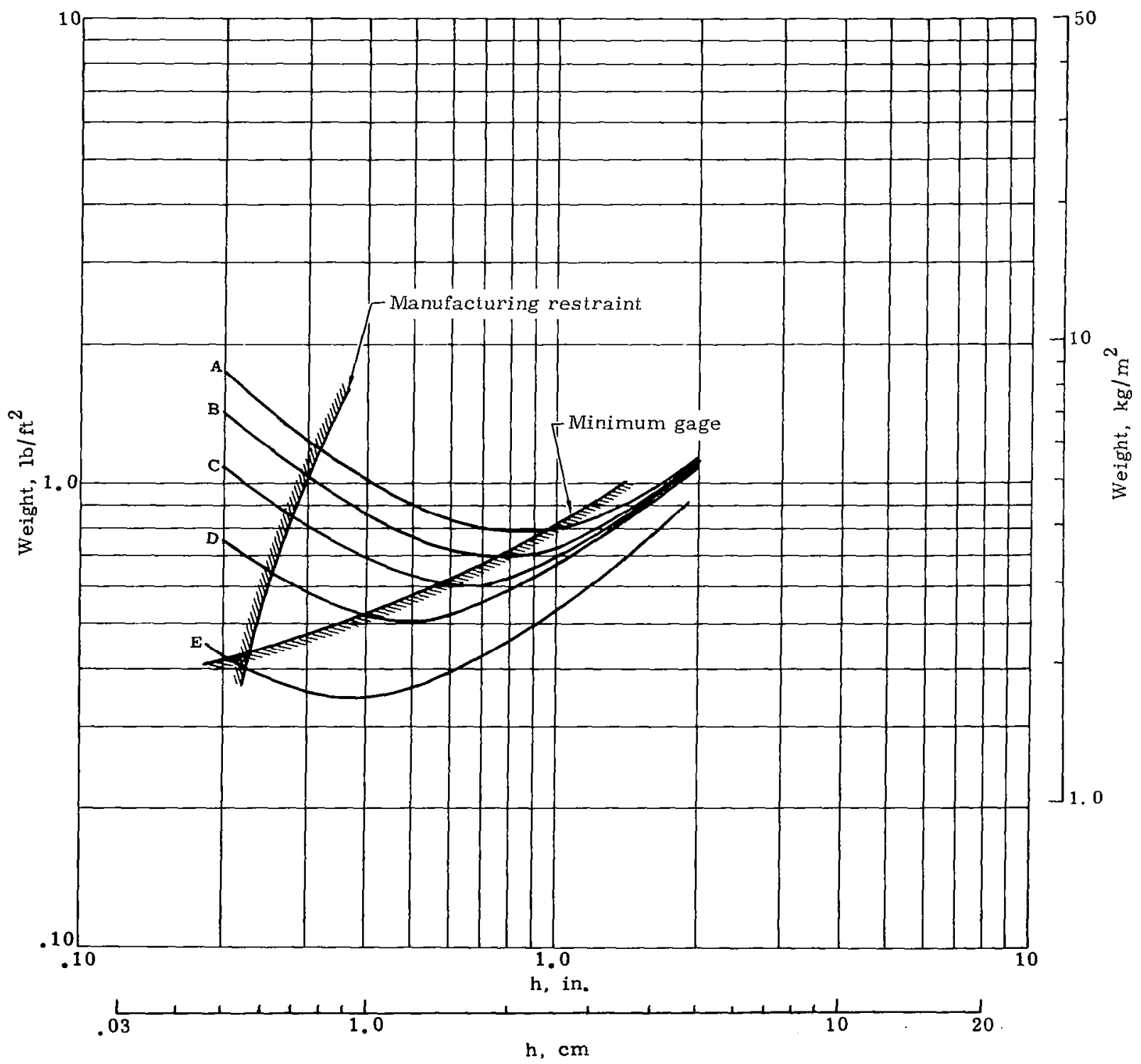

FIGURE 90. SUBSTRATE PANEL DESIGN OPTIMIZATION CURVES [PHENOLIC/GLASS SUBSTRATE PANEL, $300^{\circ} \mathrm{F}\left(422.2^{\circ} \mathrm{K}\right)$ CONDITION] 
Moment

\begin{tabular}{|c|c|c|}
\hline $\begin{array}{l}\text { Curve } \\
\text { Code }\end{array}$ & in-lb/in. & $\mathrm{cm}-\mathrm{kN} / \mathrm{cm}$ \\
\hline A & 1000 & 4.48 \\
B & 800 & 3.58 \\
C & 600 & 2.68 \\
D & 400 & 1.79 \\
E & 200 & 0.89 \\
F & 100 & 0.44 \\
G & 50 & 0.22 \\
\hline
\end{tabular}

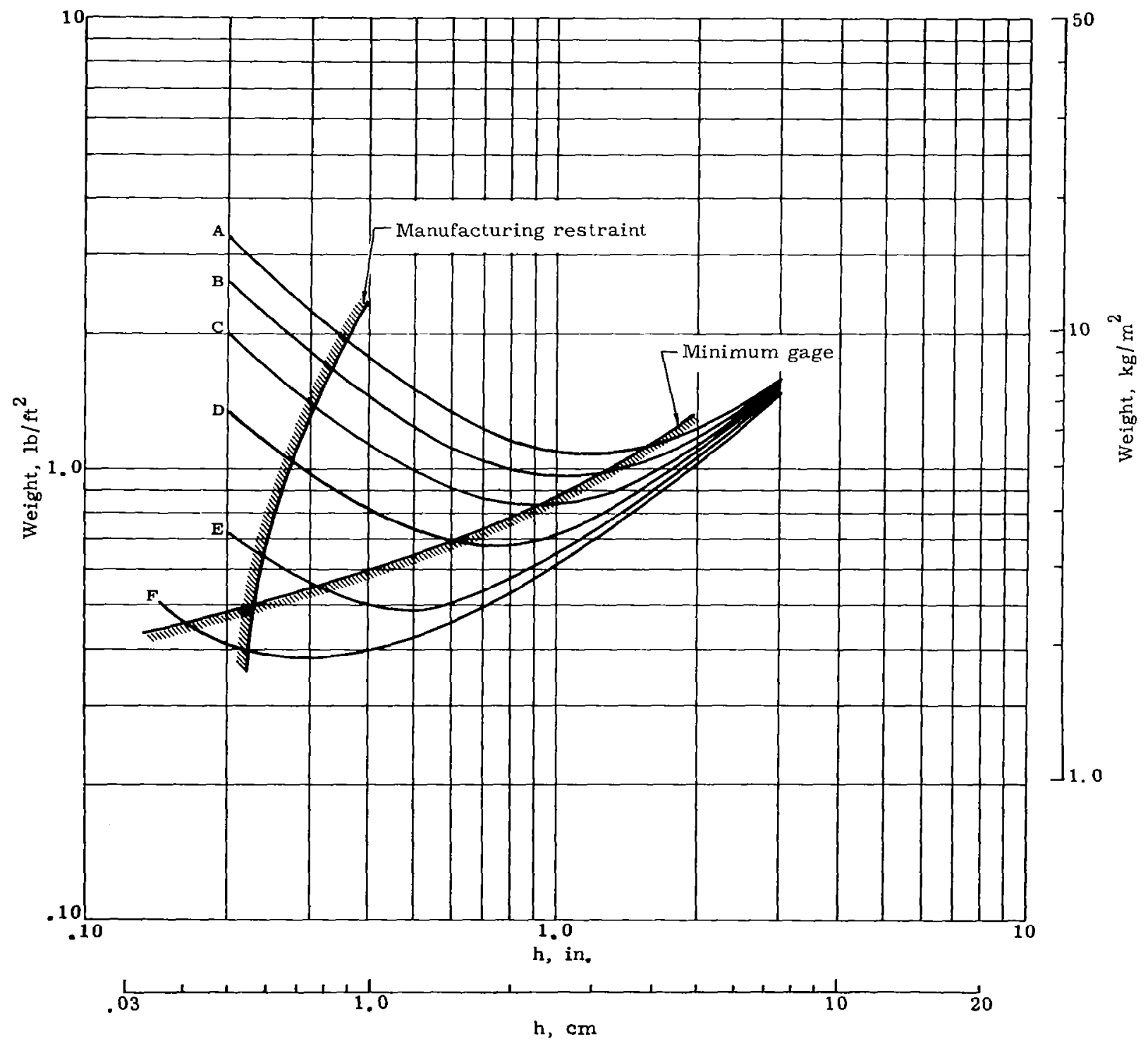

FIGURE 91. SUBSTRATE PANEL DESIGN OPTIMIZATION CURVES [PHENYL-SILANE/GLASS SUBSTRATE PANEL, $300^{\circ} \mathrm{F}\left(422.2^{\circ} \mathrm{K}\right)$ CONDITION] 


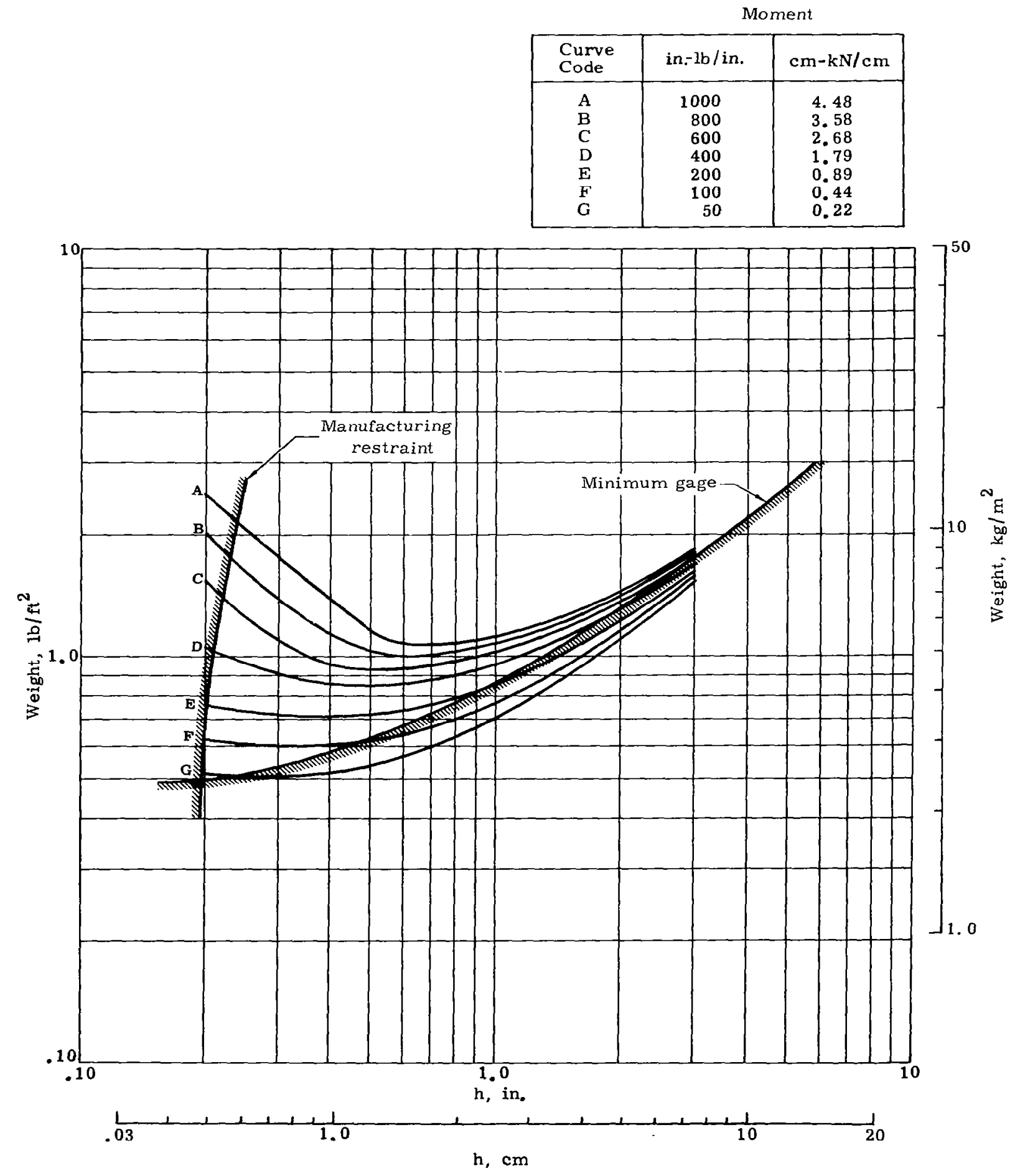

FIGURE 92. SUBSTRATE PANEL DESIGN OPTIMIZATION CURVES [17-7PH STEEL SUBSTRATE PANEL, $300^{\circ} \mathrm{F}\left(422.2^{\circ} \mathrm{K}\right)$ CONDITION] 


\begin{tabular}{|c|c|c|}
\hline \multicolumn{2}{|c|}{ Moment } \\
Curve & in-lb/in. & $\mathrm{cm}-\mathrm{kN} / \mathrm{cm}$ \\
\hline A & 1000 & 4.48 \\
B & 800 & 3.58 \\
C & 600 & 2.68 \\
D & 400 & 1.79 \\
E & 200 & 0.89 \\
F & 100 & 0.44 \\
G & 50 & 0.22 \\
\hline
\end{tabular}

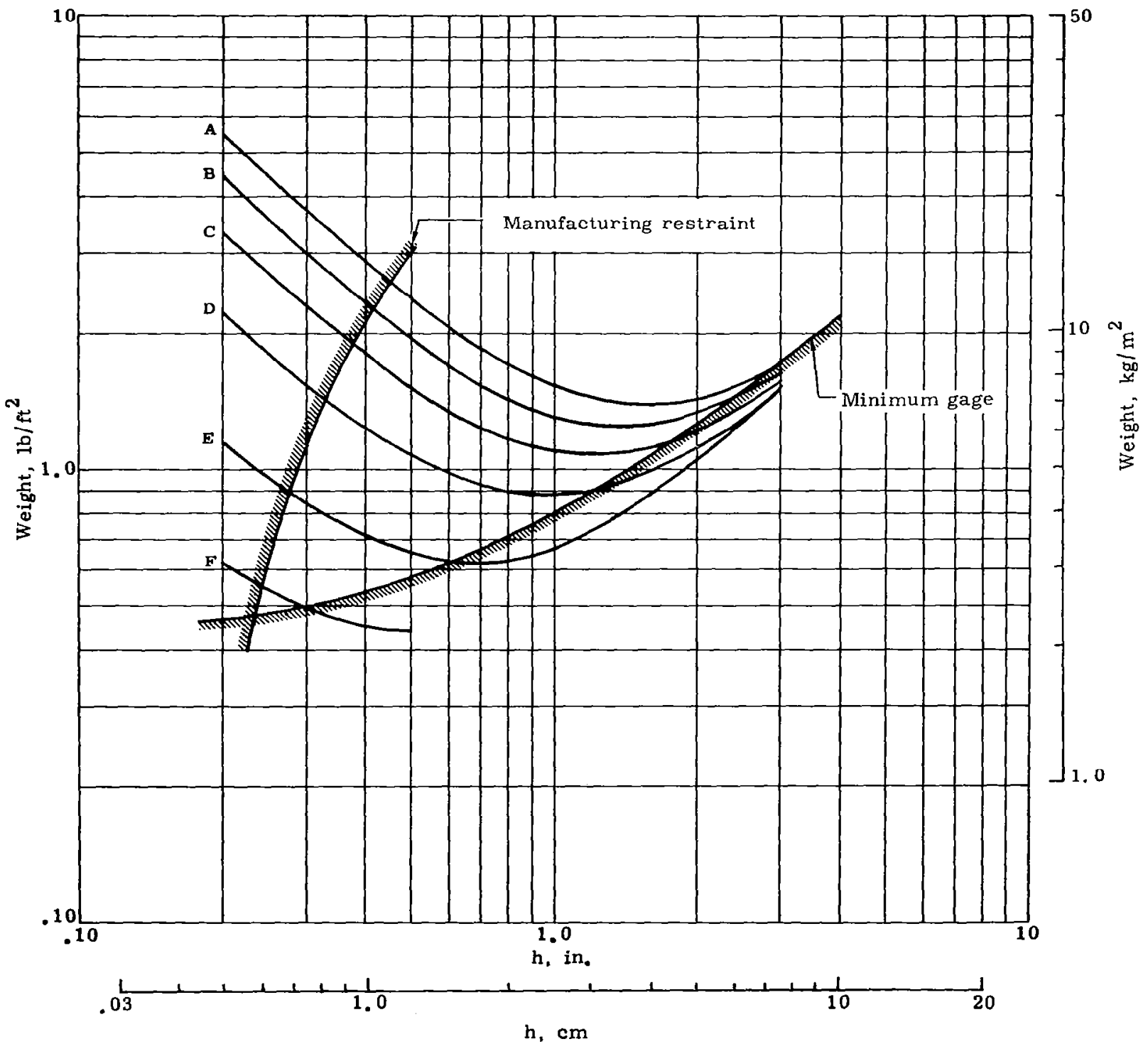

FIGURE 93. SUBSTRATE PANEL DESIGN OPTIMIZATION CURVES [PHENOLIC/GLASS SUBSTRATE PANEL, $800^{\circ} \mathrm{F}\left(700^{\circ} \mathrm{K}\right)$ CONDITION] 


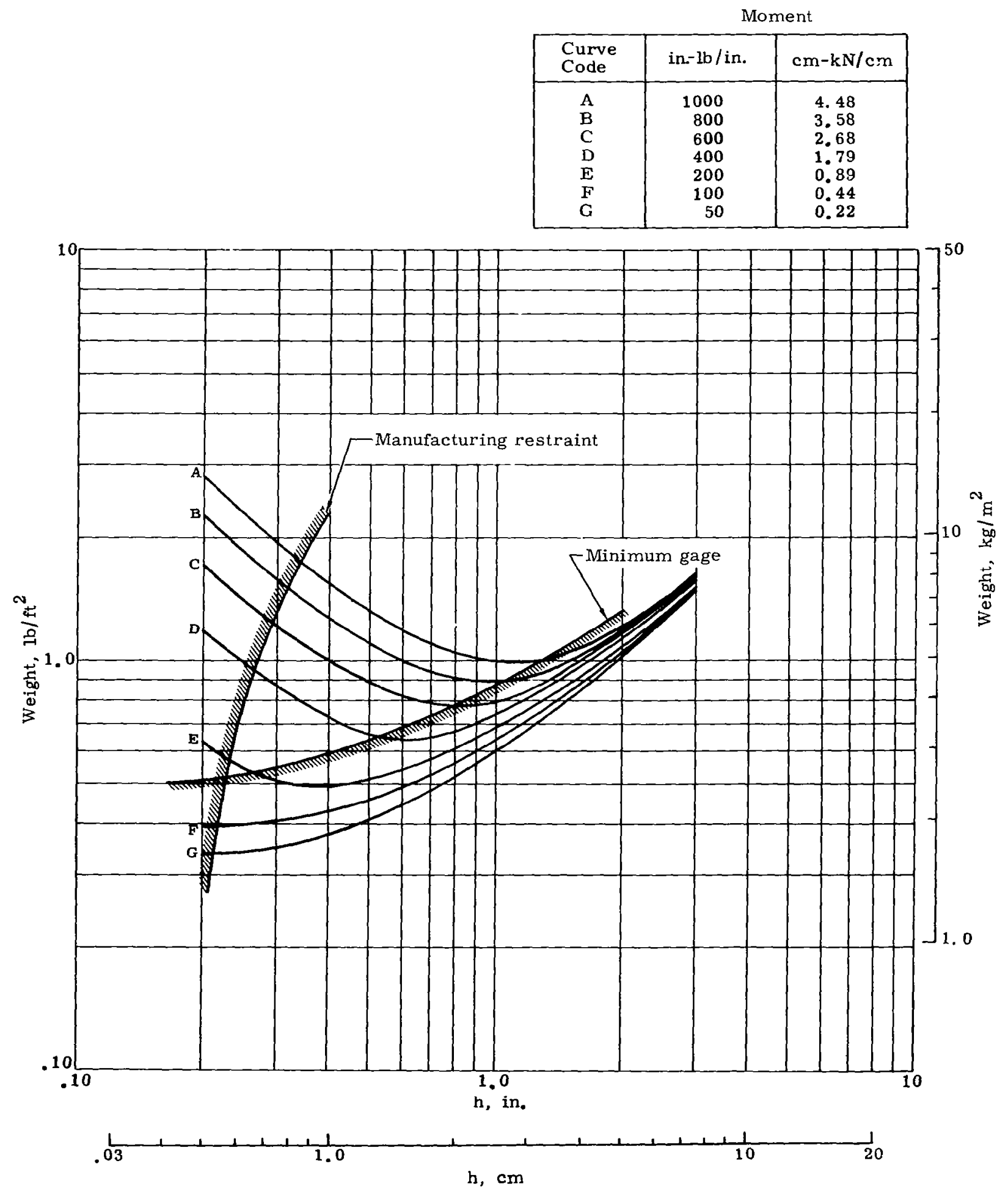

FIGURE 94. SUBSTRATE PANEL DESIGN OPTIMIZATION CURVES [PHENYL-SILANE/GLASS SUBSTRATE PANEL, $800^{\circ} \mathrm{F}\left(700^{\circ} \mathrm{K}\right)$ CONDITION] 


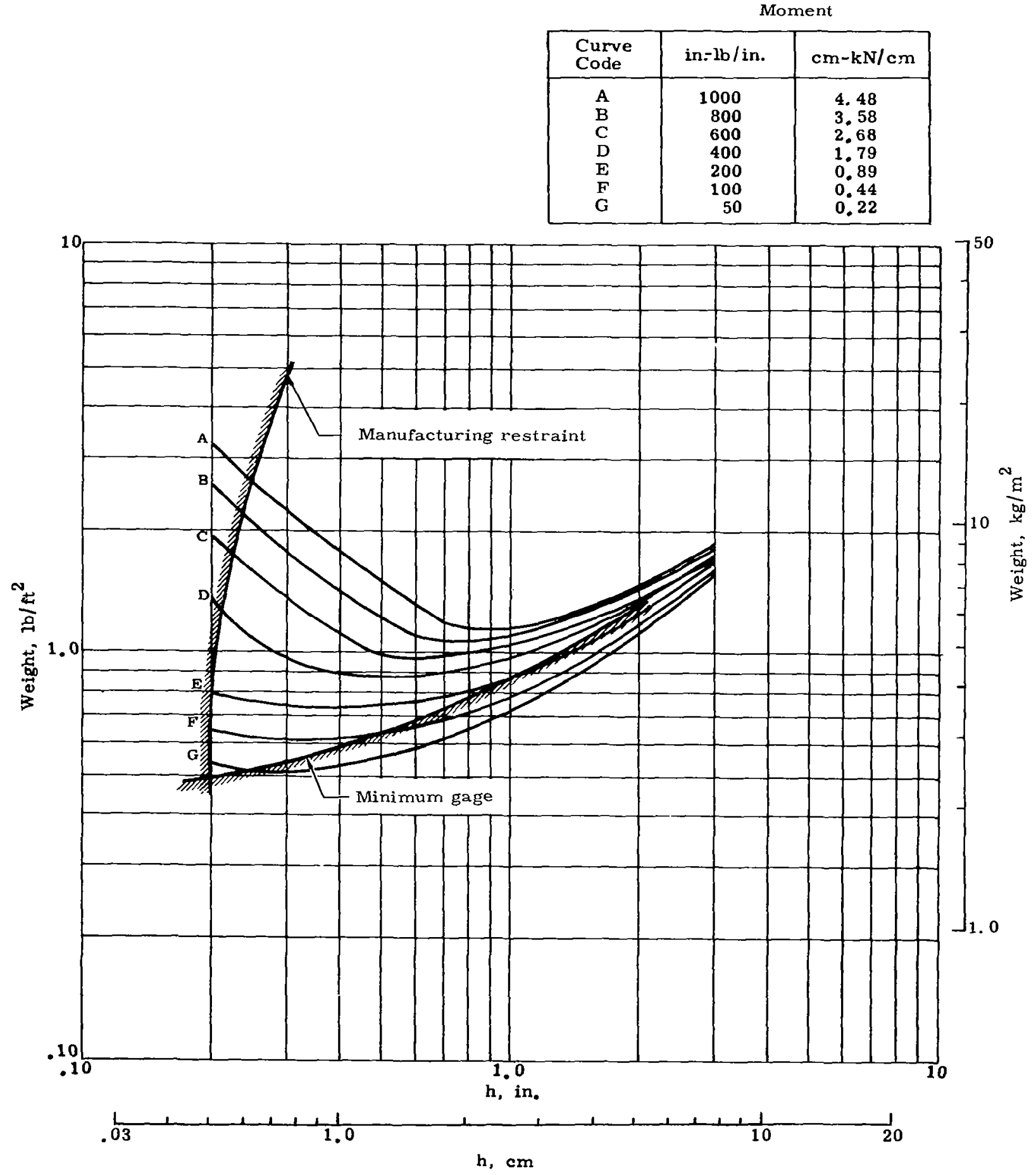

FIGURE 95. SUBSTRATE PANEL DESIGN OPTIMIZAT ҐON CURVES [17-7PH STEEL SUBSTRATE PANEL, $800^{\circ} \mathrm{F}\left(700^{\circ} \mathrm{K}\right)$ CONDITION] 


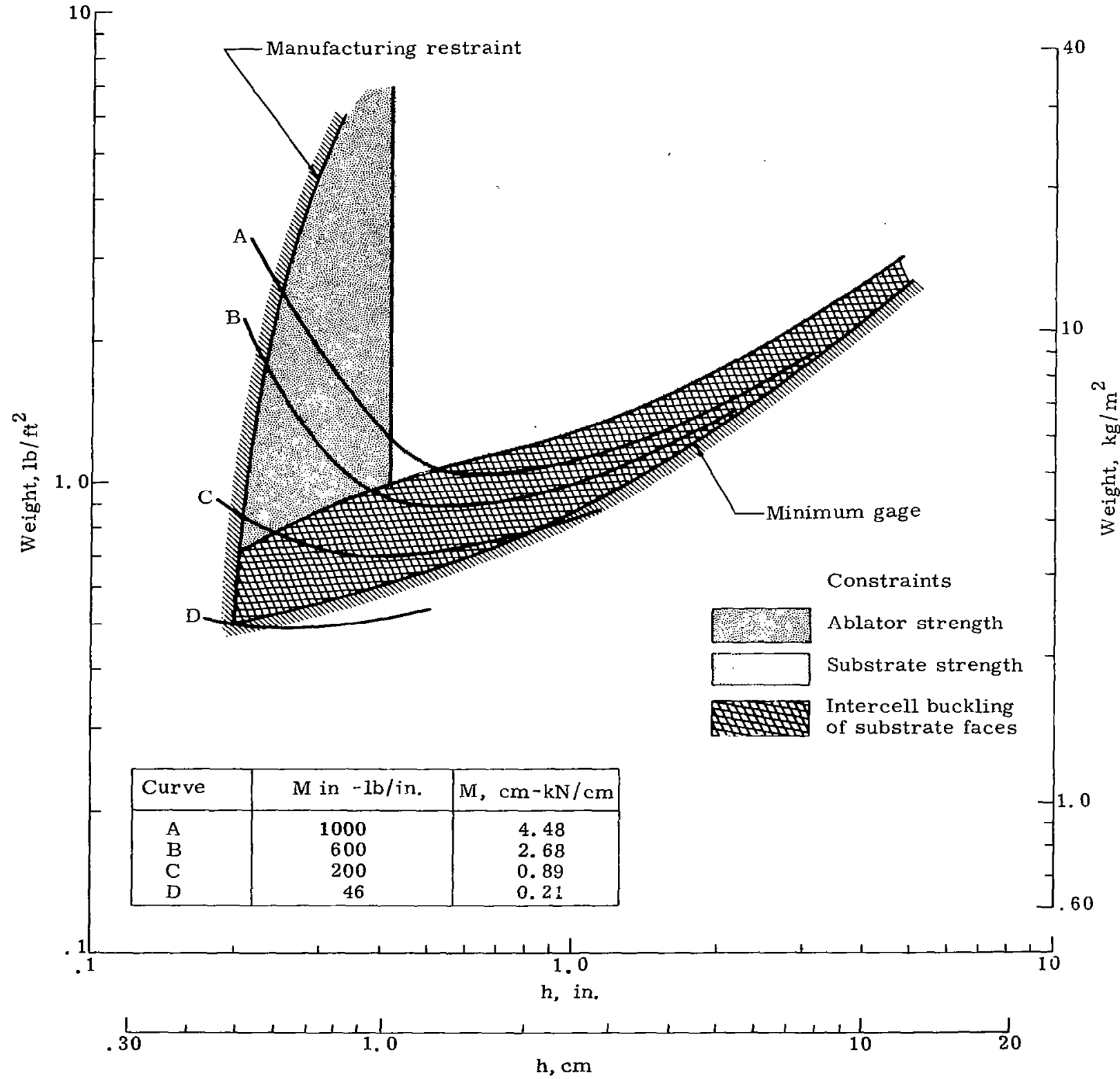

FIGURE 96. DESIGN CONSTRAINT RELATIONSHIPS [17-7PH STEEL SUBSTRATE WITH $1.50-$ IN. $(3.81 \mathrm{CM})$ NASA 602 ABLATOR, $70^{\circ} \mathrm{F}\left(294^{\circ} \mathrm{K}\right)$ CONDITION] 


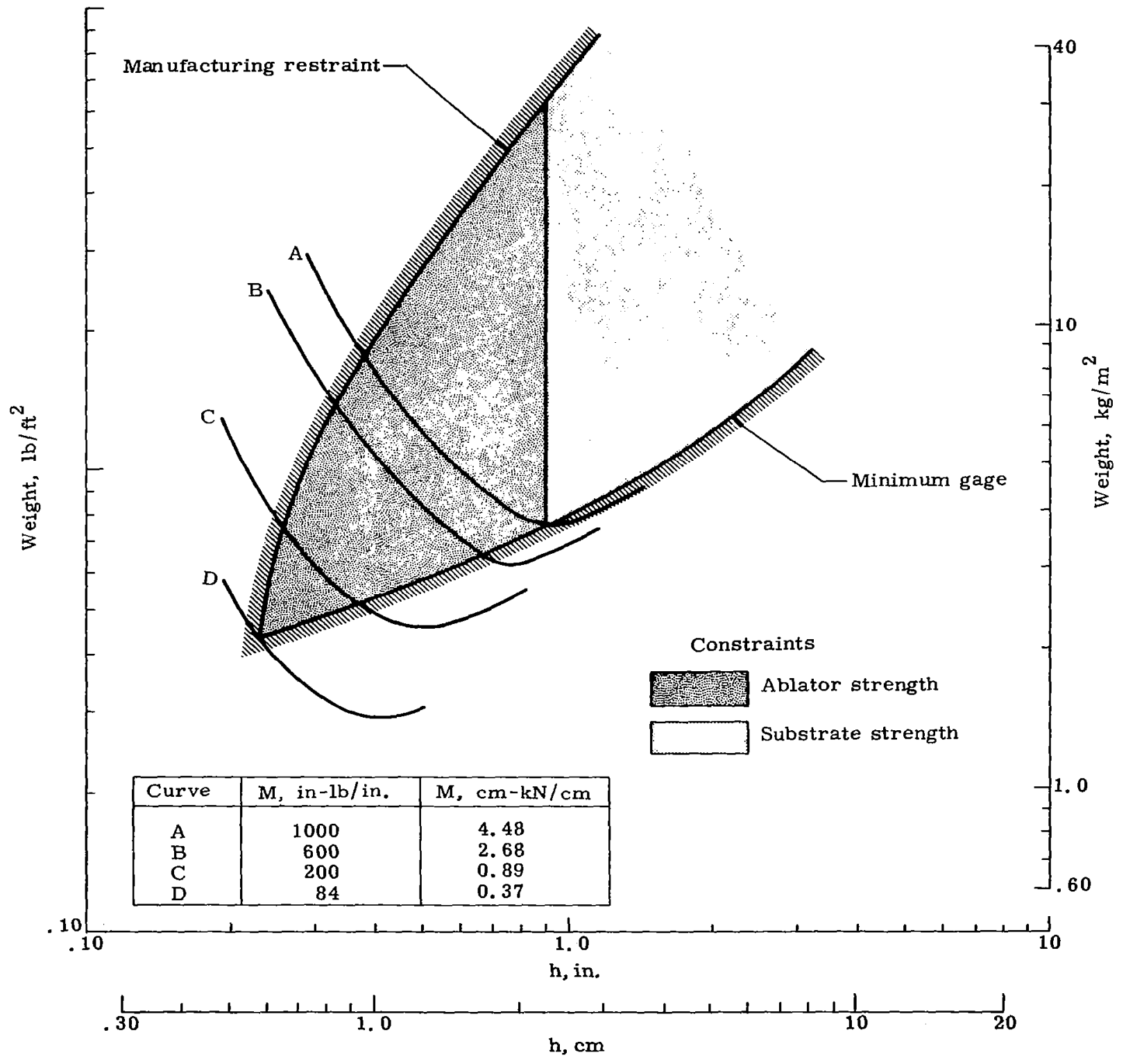

FIGURE 97. DESIGN CONSTRAINT RELATIONSHIPS [PHENOLIC/GLASS SUBSTRATE WITH 1.50-IN. $(3.81 \mathrm{CM})$ NASA 602 ABLATOR, $70^{\circ} \mathrm{F}\left(294.4^{\circ} \mathrm{K}\right)$ CONDITION] 


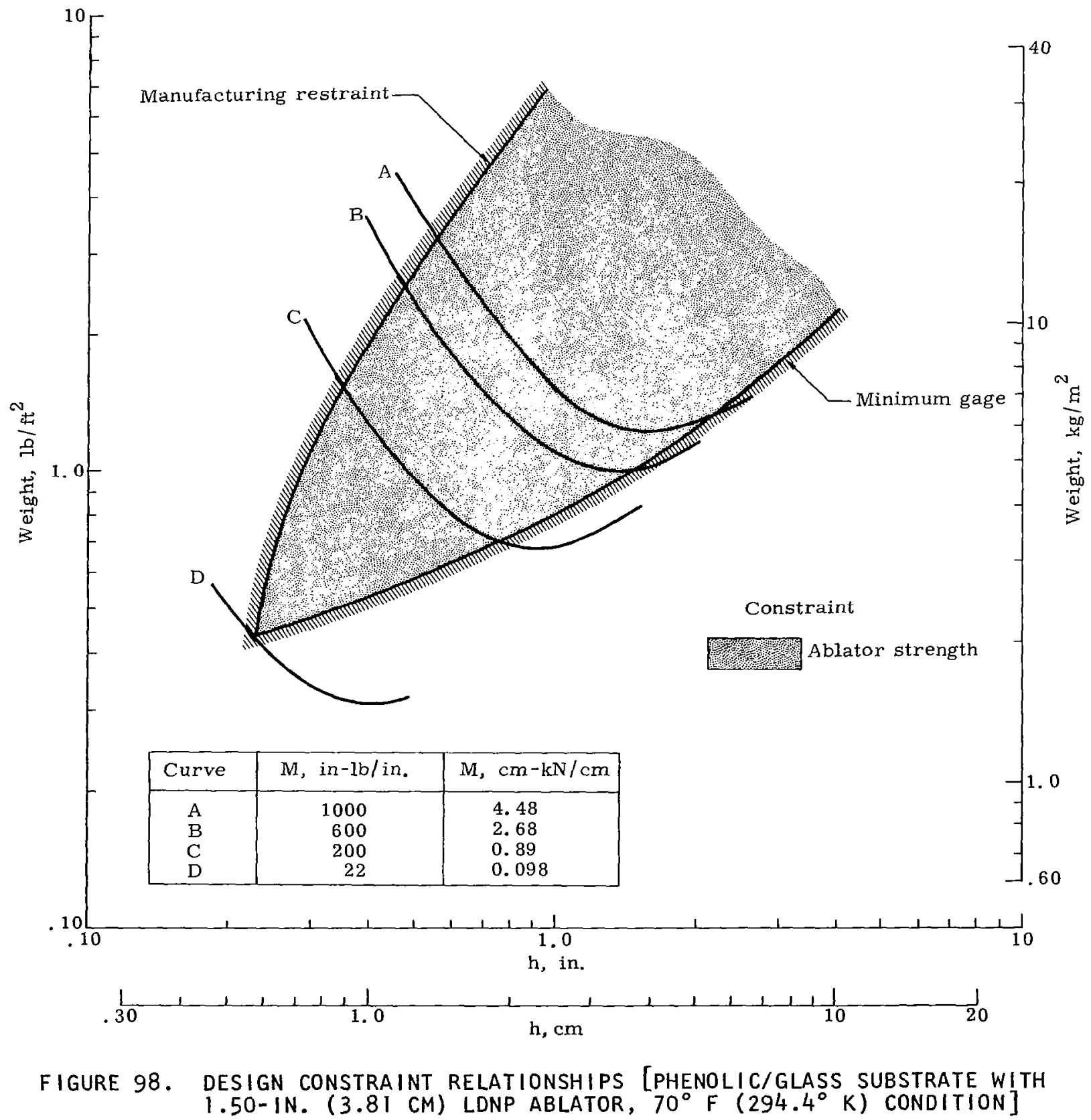




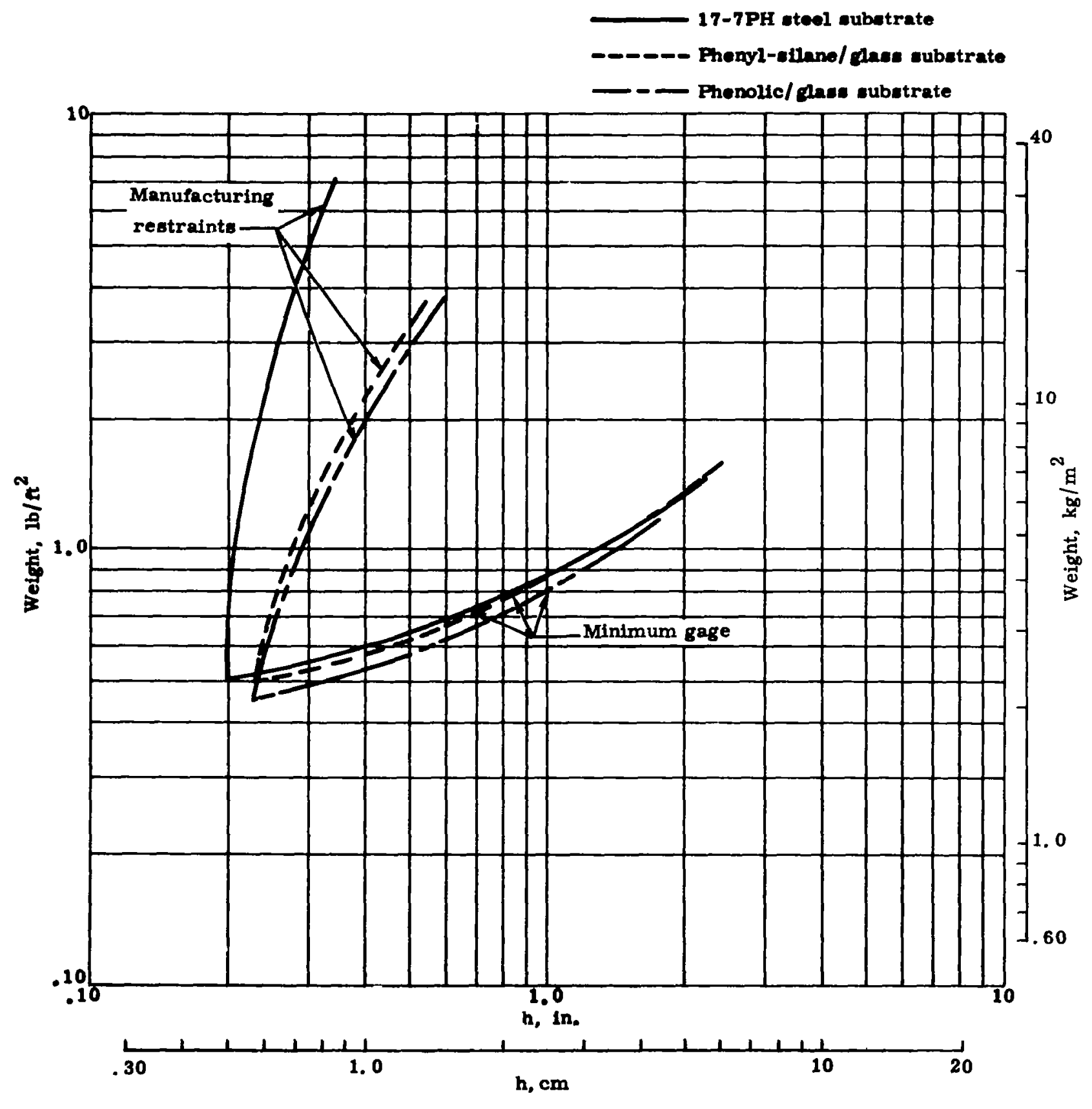

FIGURE 99. SUBSTRATE PANEL DESIGN CORRIDORS 


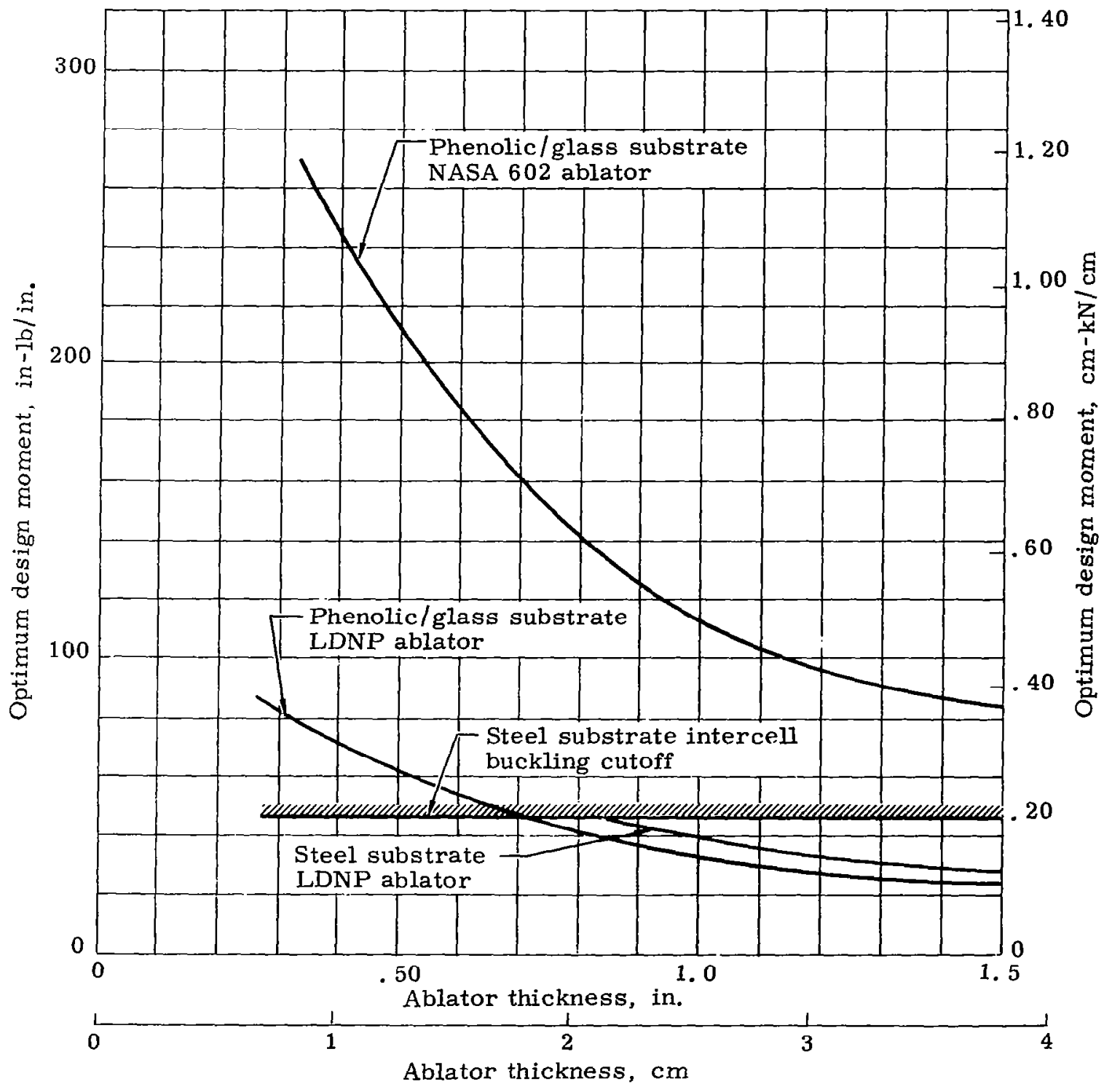

FIGURE 100. OPTIMUM DESIGN MOMENTS FOR SUBSTRATE/ABLATOR COMBINATIONS $\left[70^{\circ} \mathrm{F}\left(294.4^{\circ} \mathrm{K}\right)\right.$ CONDITION] 


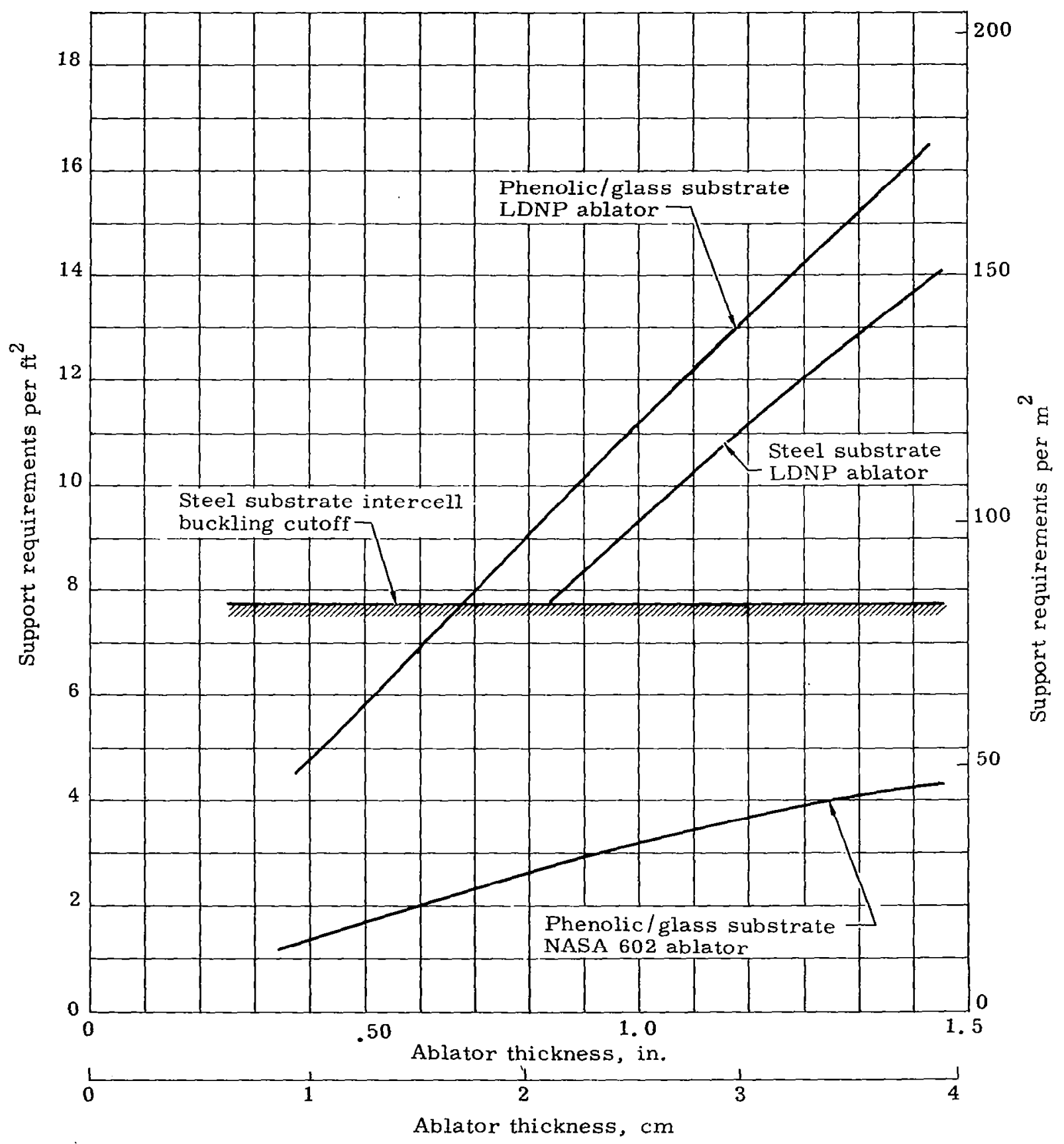

FIGURE 101. PANEL SUPPORT REQUIREMENTS FOR OPTIMUM MOMENTS $\left[70^{\circ} \mathrm{F}\left(294.4^{\circ} \mathrm{K}\right)\right.$ CONDITION, $12 \mathrm{PSI}\left(82.74 \mathrm{kN} / \mathrm{m}^{2}\right)$ PRESSURE] 


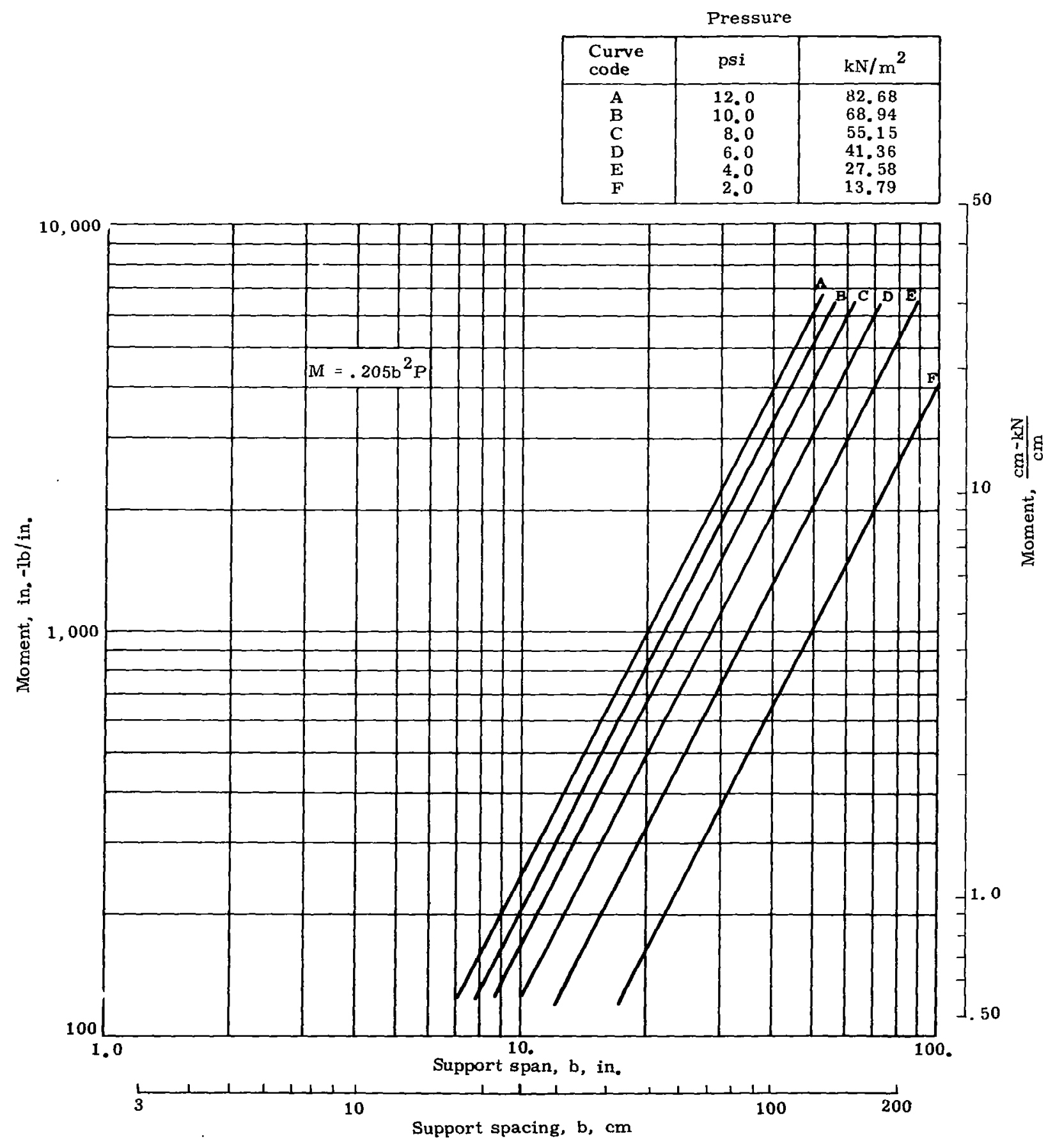

FIGURE 102. CRITICAL PANEL MOMENT VERSUS SUPPORT SPAN AND PRESSURE ASPECT RATIO $=1.0=a / b$ 
Pressure

\begin{tabular}{|c|c|c|}
\hline $\begin{array}{l}\text { Curve } \\
\text { code }\end{array}$ & psi & $\mathrm{kN} / \mathrm{m}^{2}$ \\
\hline A & 12.0 & 82.68 \\
B & 10.0 & 68.94 \\
C & 8.0 & 55.15 \\
D & 6.0 & 41.36 \\
E & 4.0 & 27.58 \\
F & 2.0 & 13.79 \\
\hline
\end{tabular}

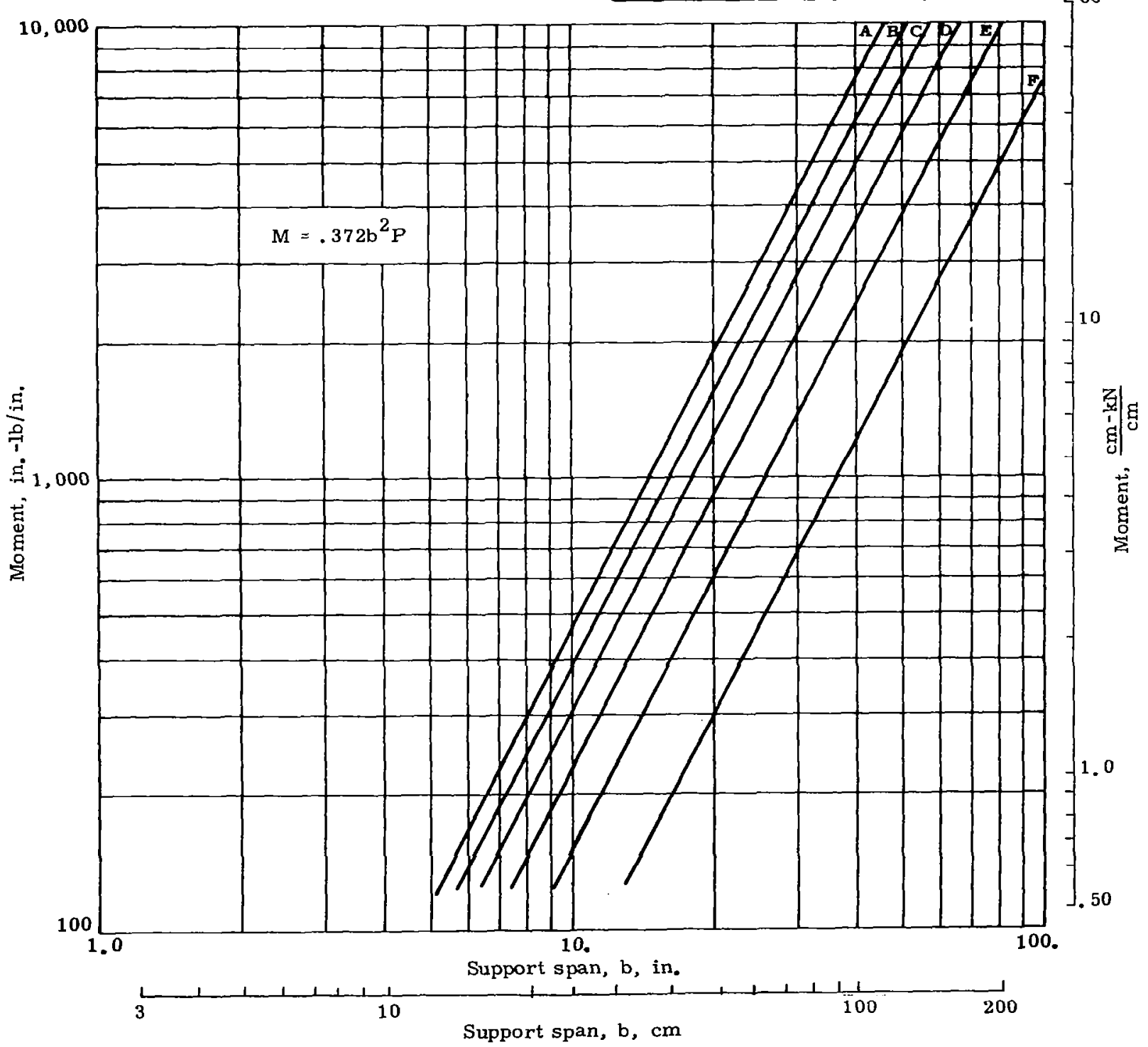

FIGURE 103. CRITICAL PANEL MOMENT VERSUS SUPPORT SPAN AND PRESSURE ASPECT RATIO $=1.5=a / b$ 


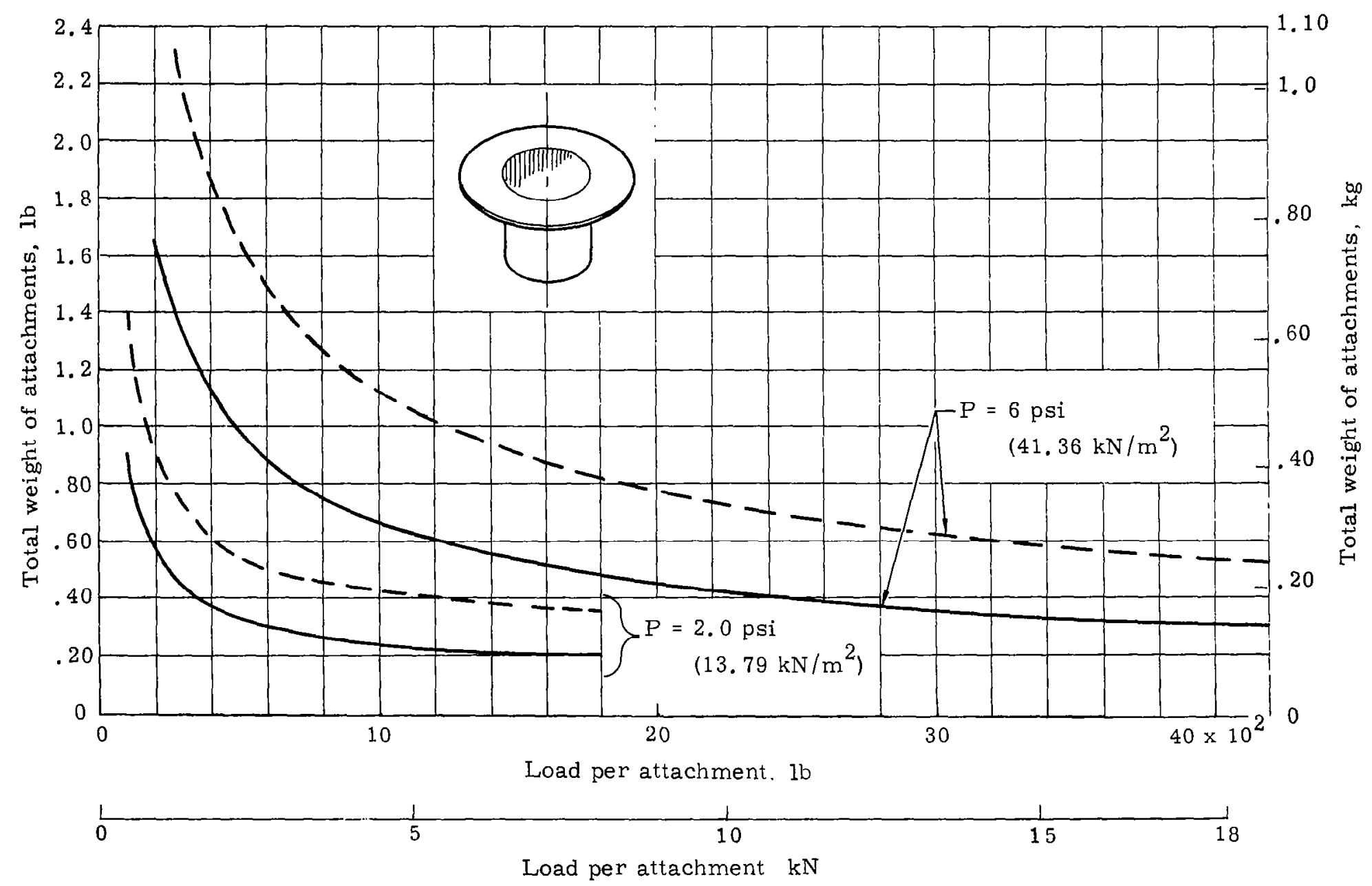




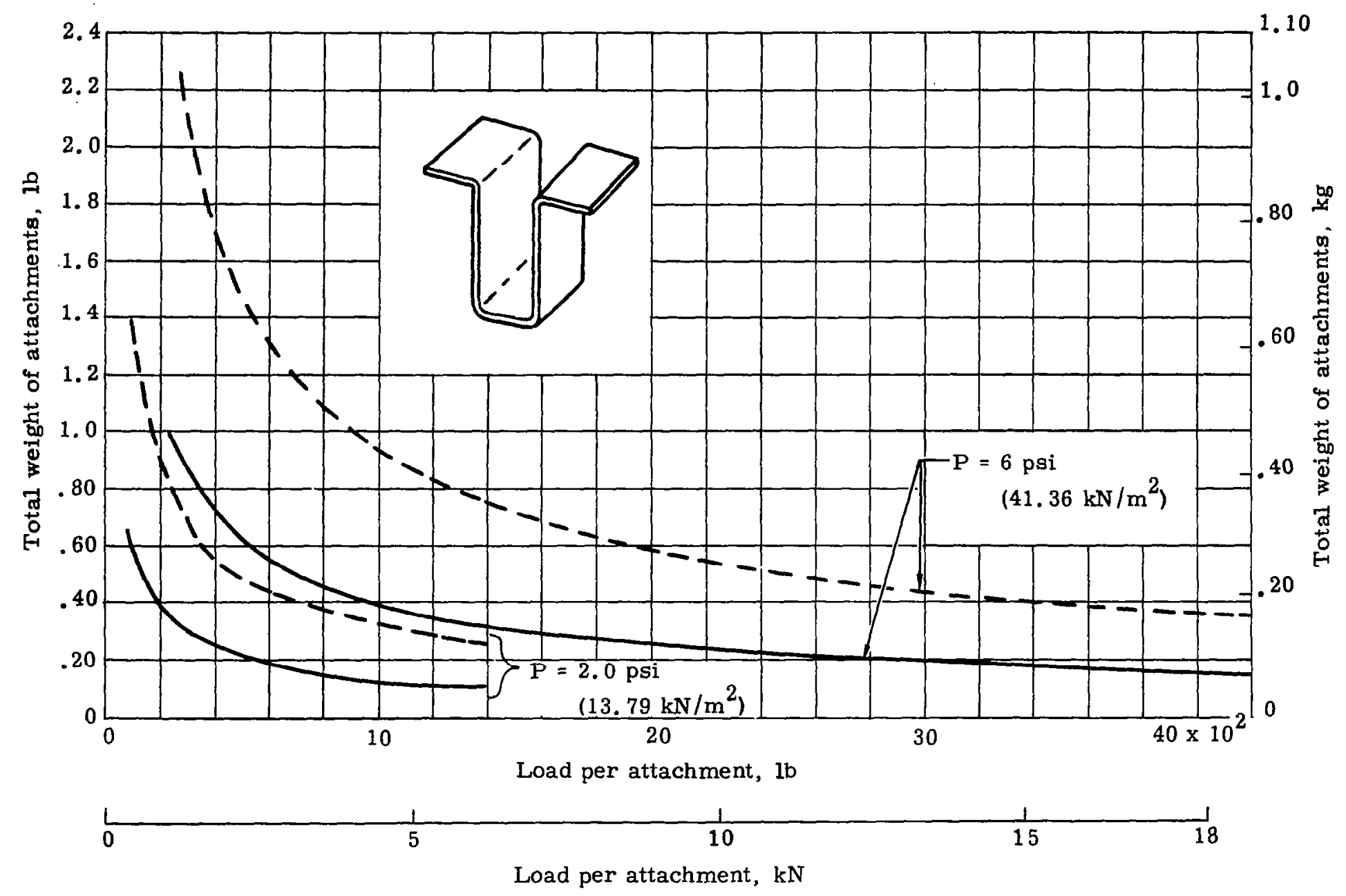




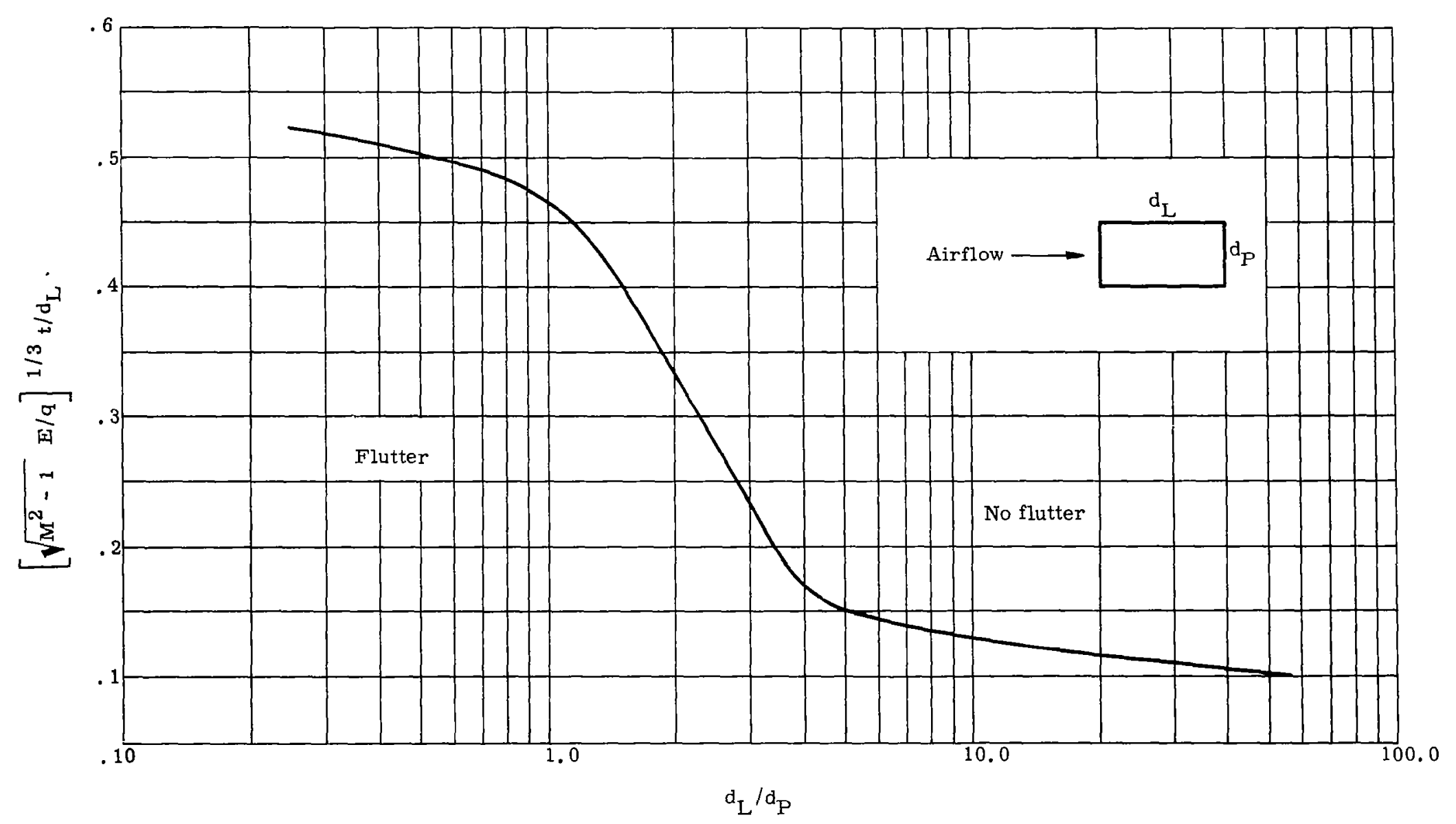

FIGURE 106. FLUTTER BOUNDARY CRITERIA 

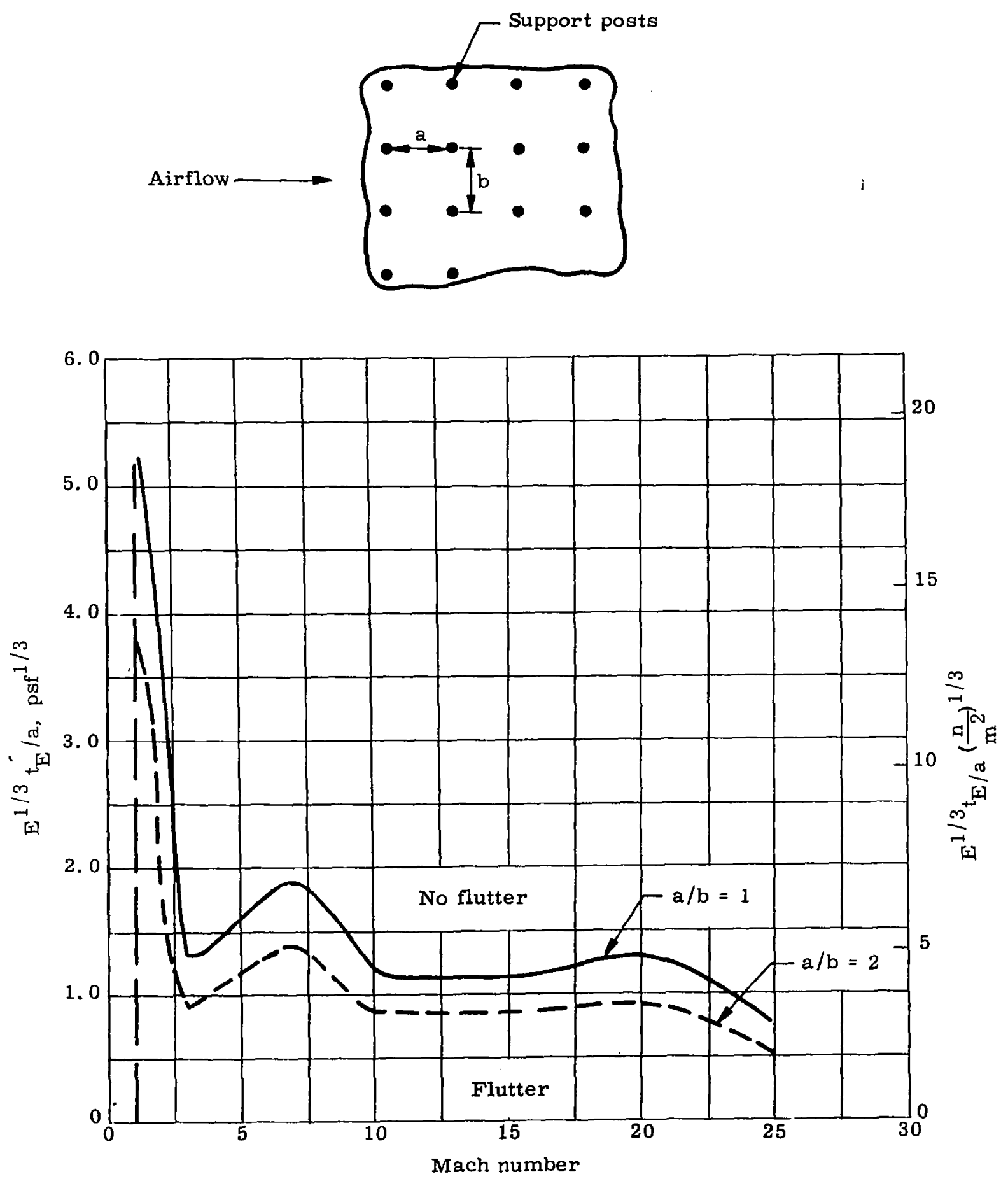

FIGURE 107. HL-10 FLUTTER ENVELOPE 
1

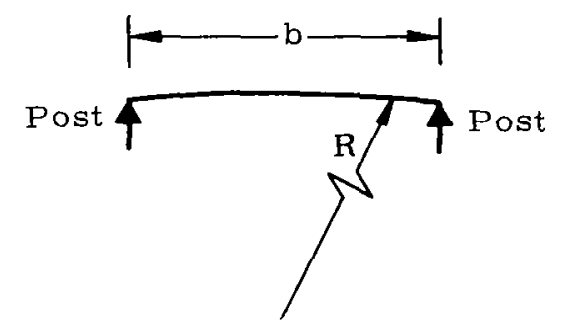

$t_{E}=t x$ curvature correction factor

$t_{E}=$ thickness of equivalent flat plate

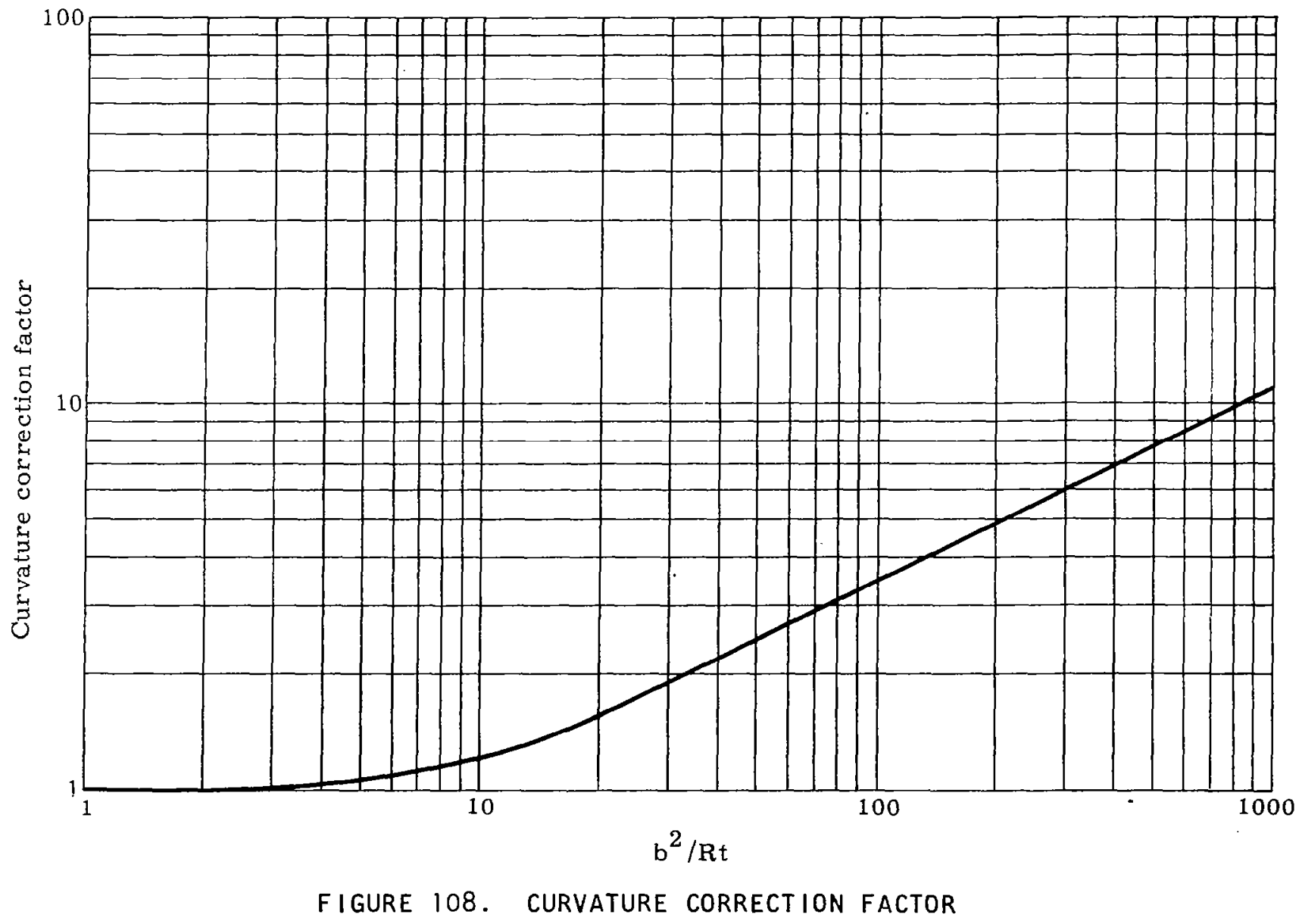



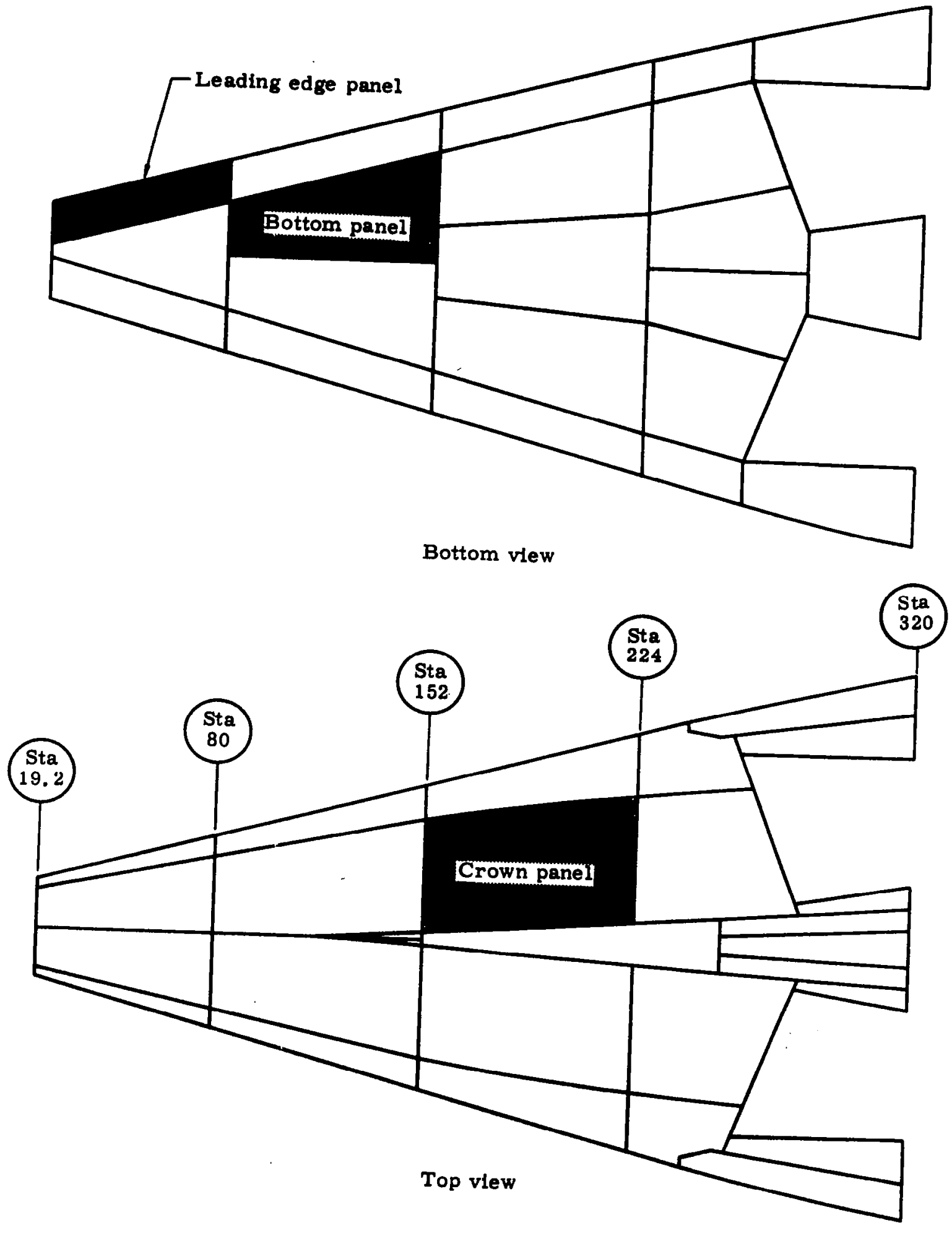

FIGURE 109. HEAT SHIELD PANEL LOCATIONS 


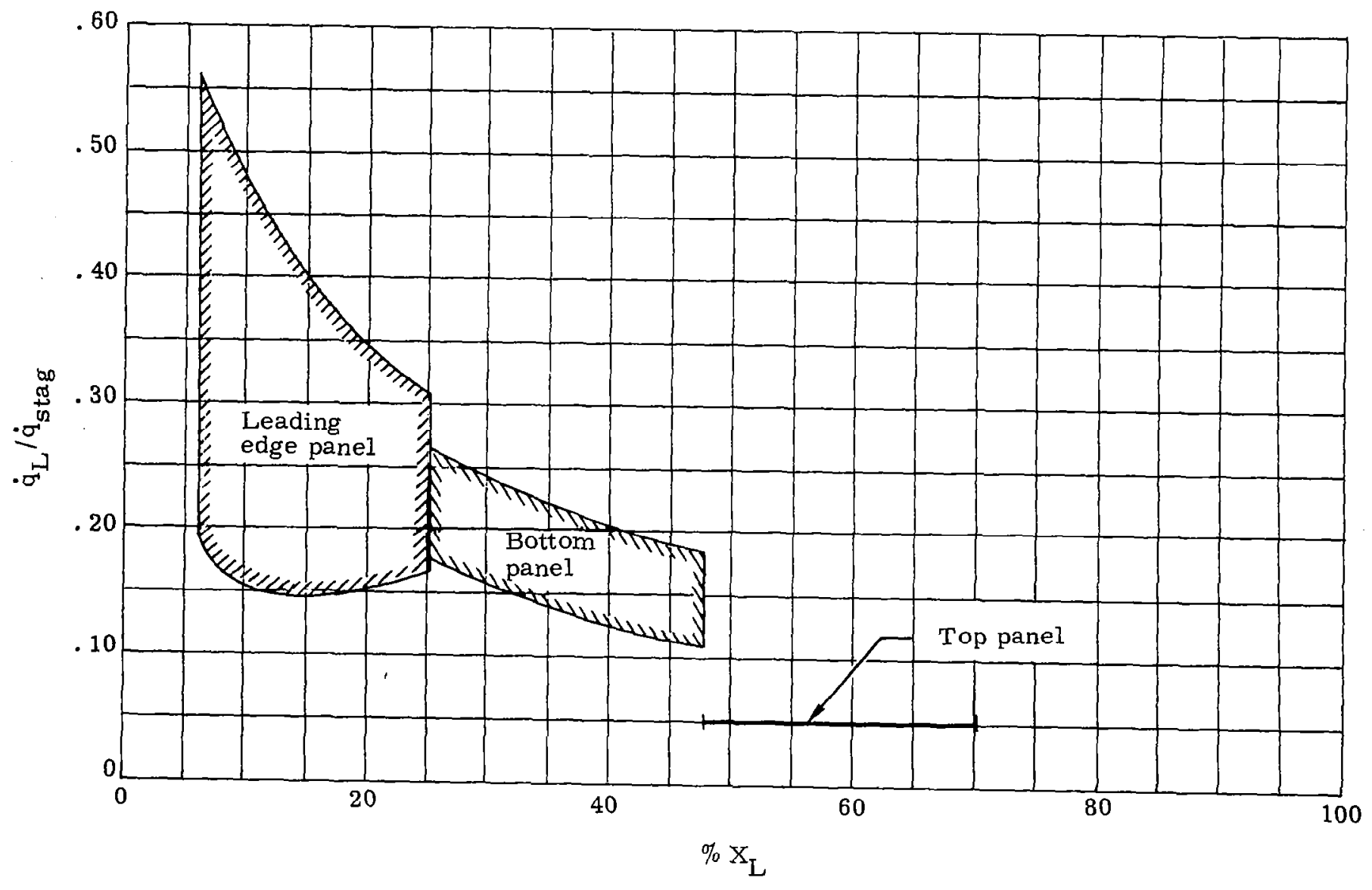

FIGURE 110. HEATING RATE RATIO ENVELOPES FOR PANEL DESIGN [NOMINAL RE-ENTRY TRAJECTORY (LAMINAR FLOW)] 


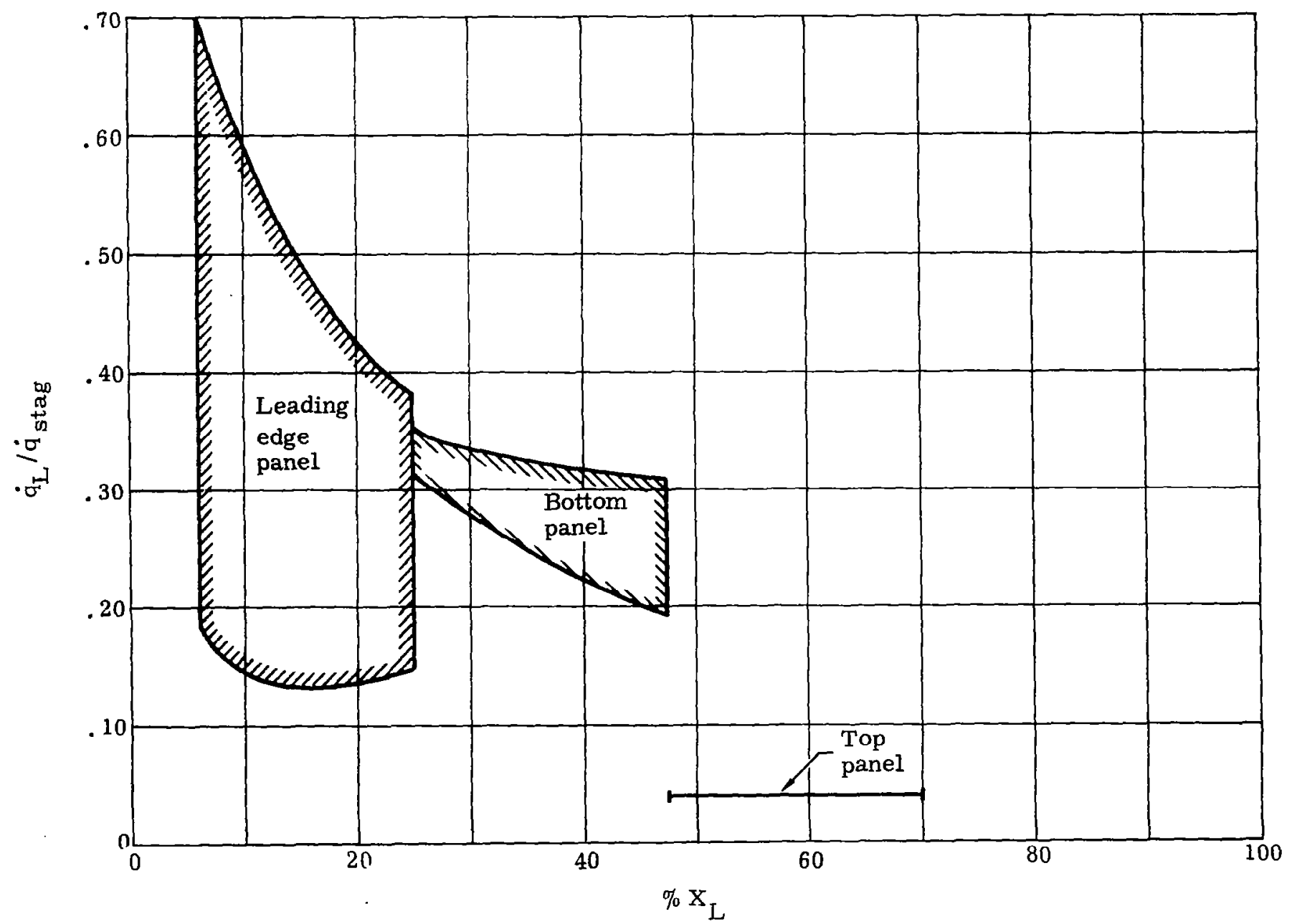

FIGURE 111. HEATING RATE RATIO ENVELOPES FOR PANEL DESIGN[OVERSHOOT RE-ENTRY TRAJECTORY (LAMINAR FLOW)] 


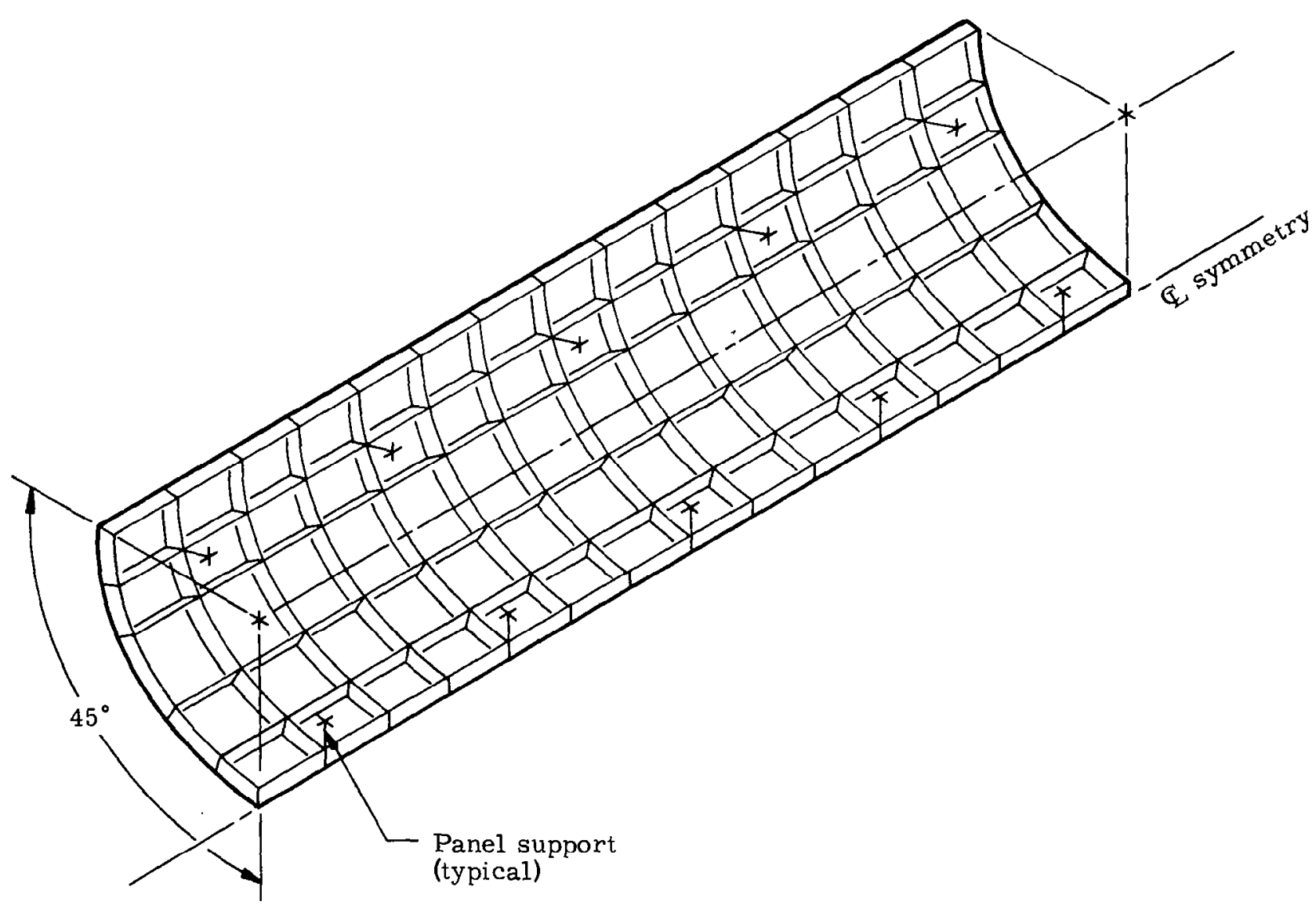

FIGURE 112 LEADING EDGE PANEL MODEL FOR STRUCTURAL NETWORK ANALYSIS 


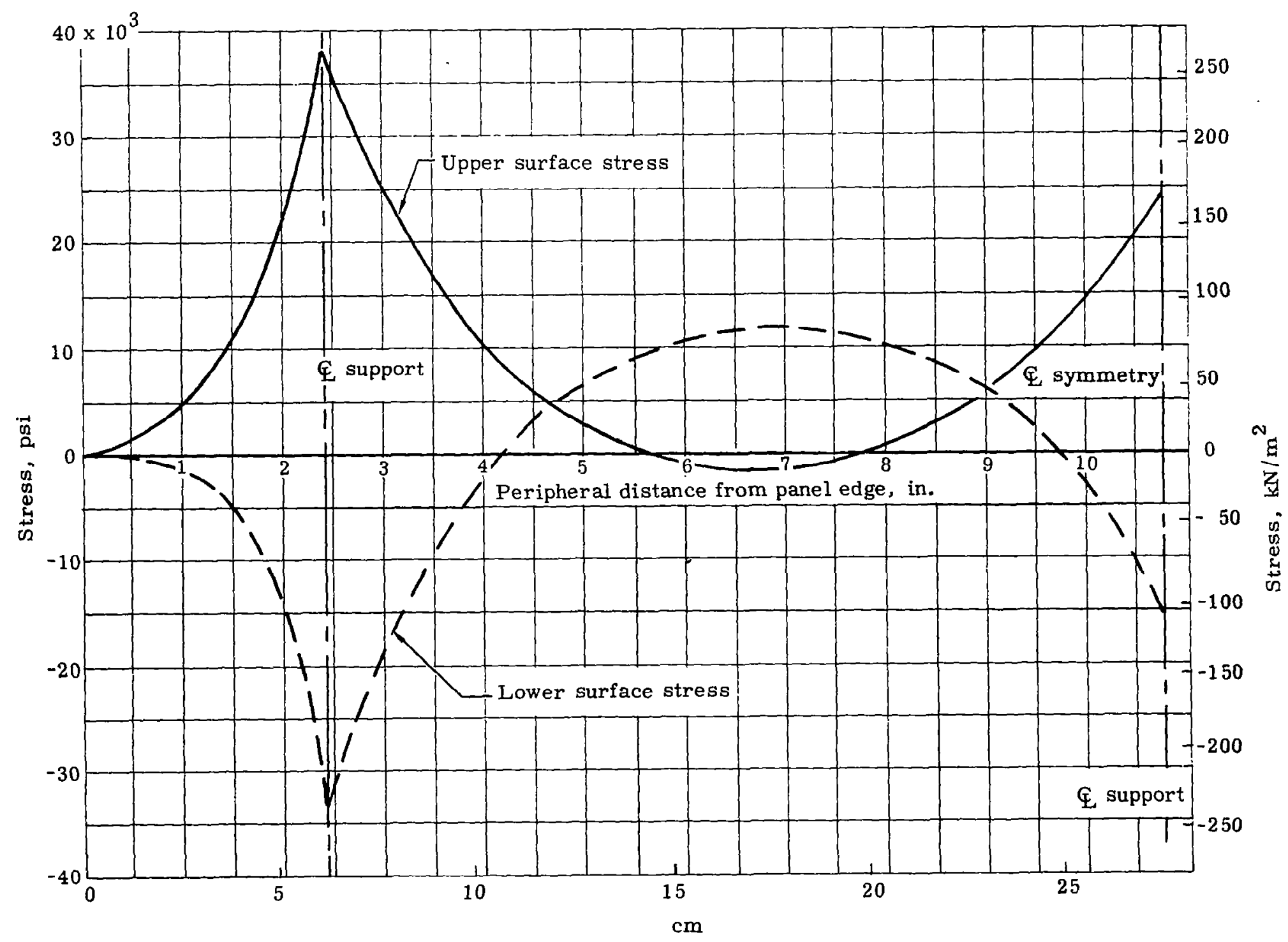

FIGURE 113. LEADING EDGE SUBSTRATE PANEL, ULTIMATE PANEL FACE STRESSES (MAXIMUM LOAD FACTOR, ABORT CONOITION) 


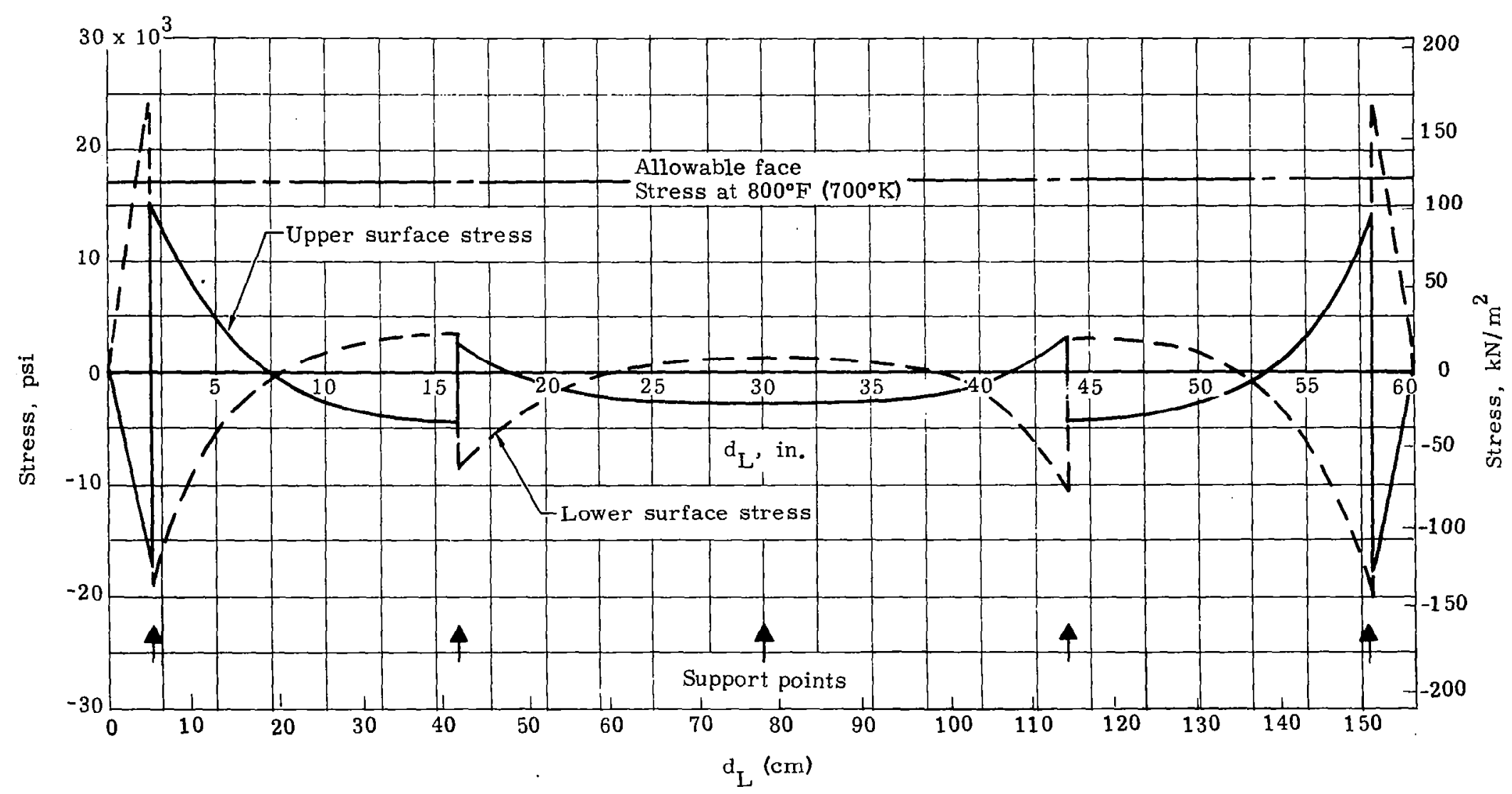

FIGURE 114. LEADING EDGE SUBSTRATE PANEL-CRITICAL THERMAL CONDITION STRESSES 


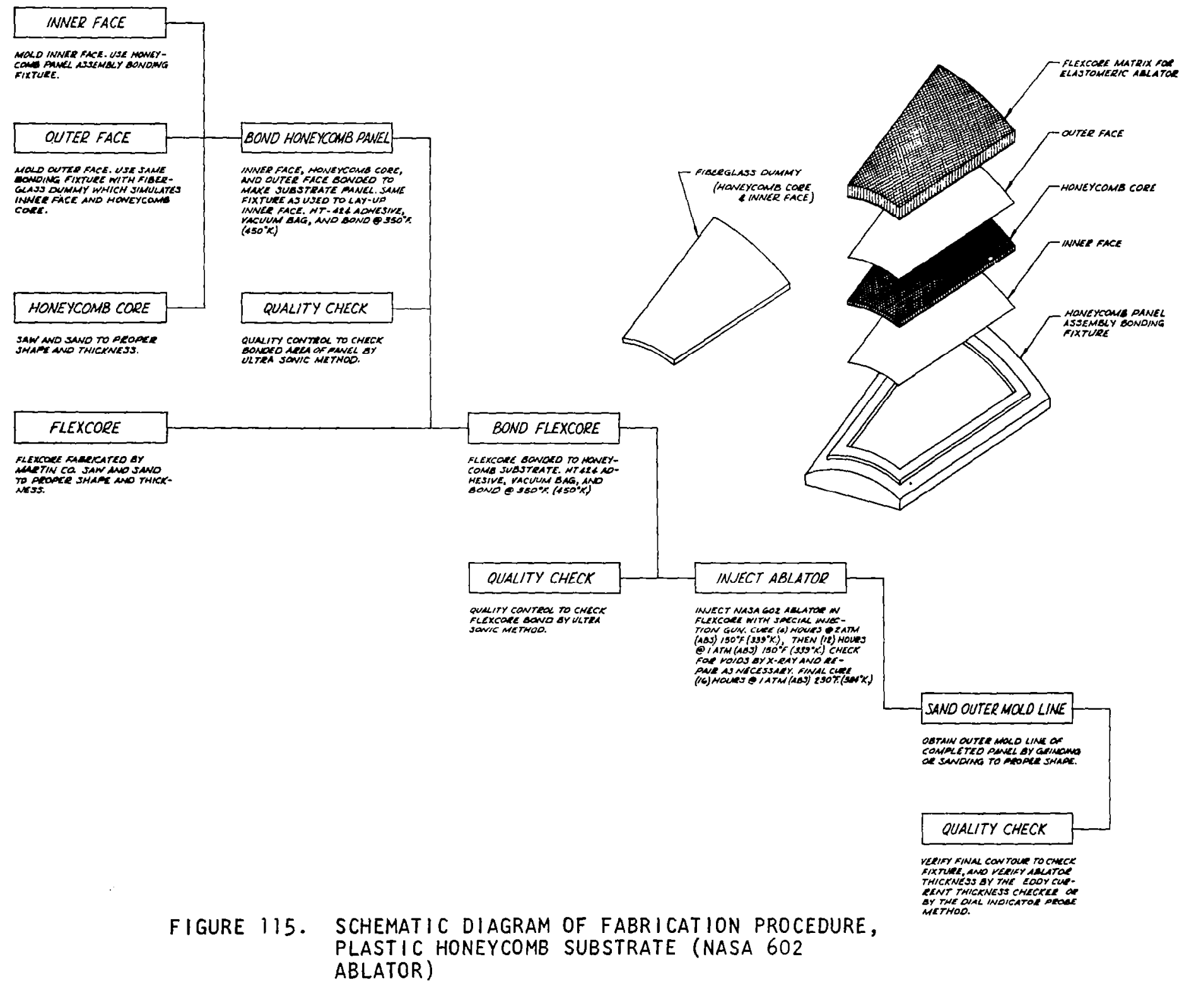


趈

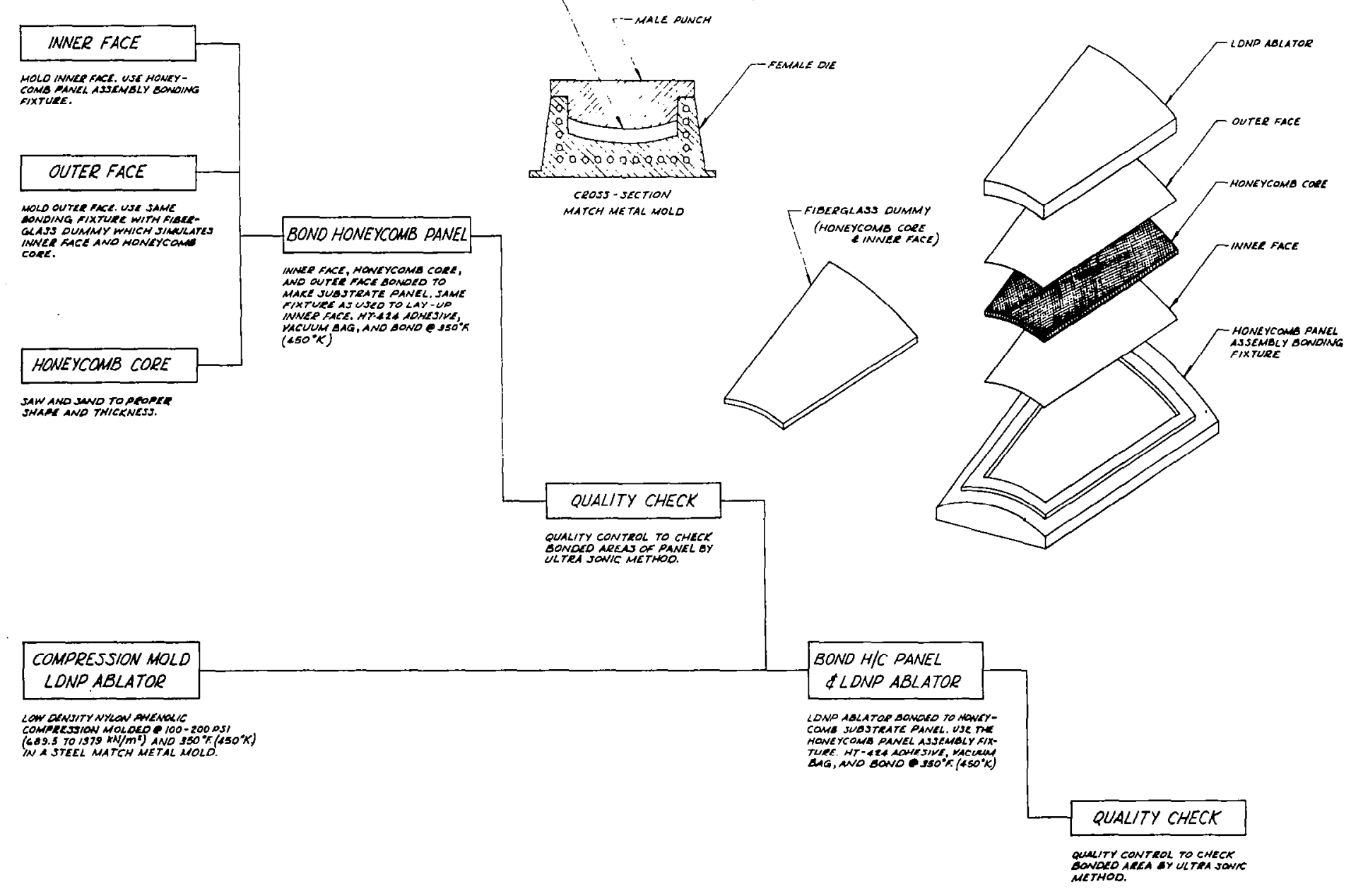

FIGURE 116. SCHEMATIC DIAGRAM OF FABRICATION PROCEDURE (PLAST I C HONEYCOMB SUBSTRATE, LDNP ABLATOR) 


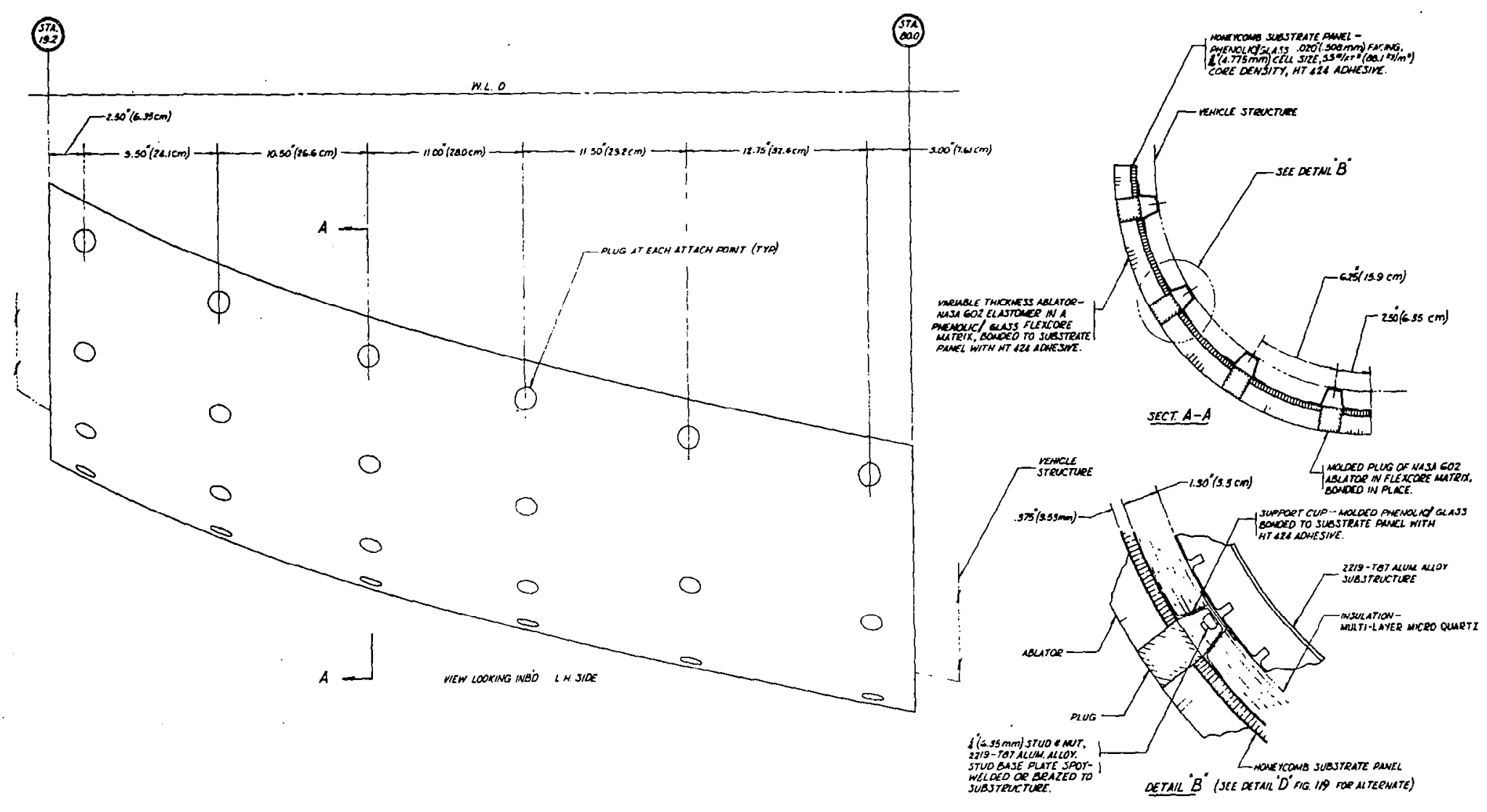

FIGURE 117. LEADING EDGE PANEL 


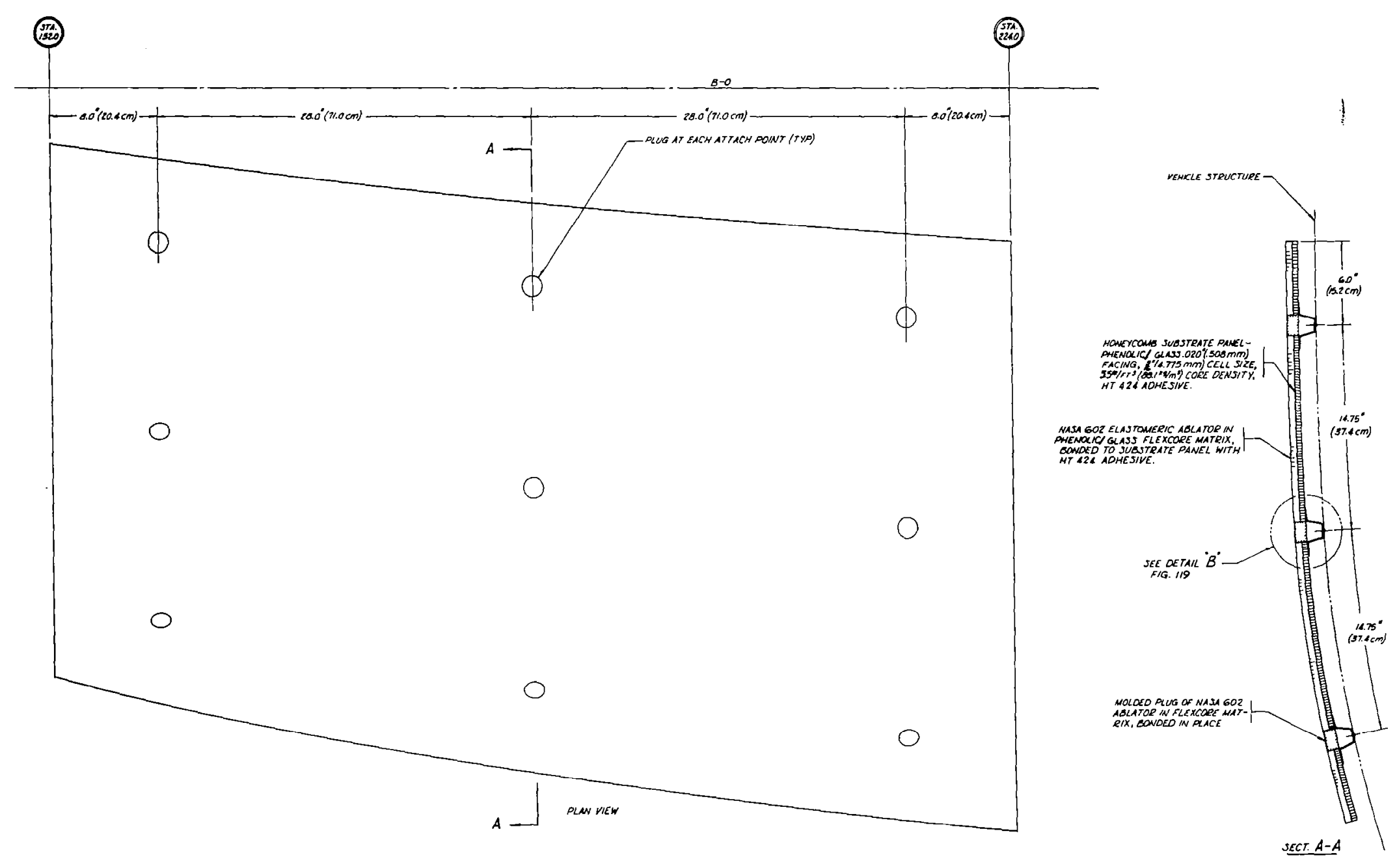

FIGURE 118. CROWN PANEL 

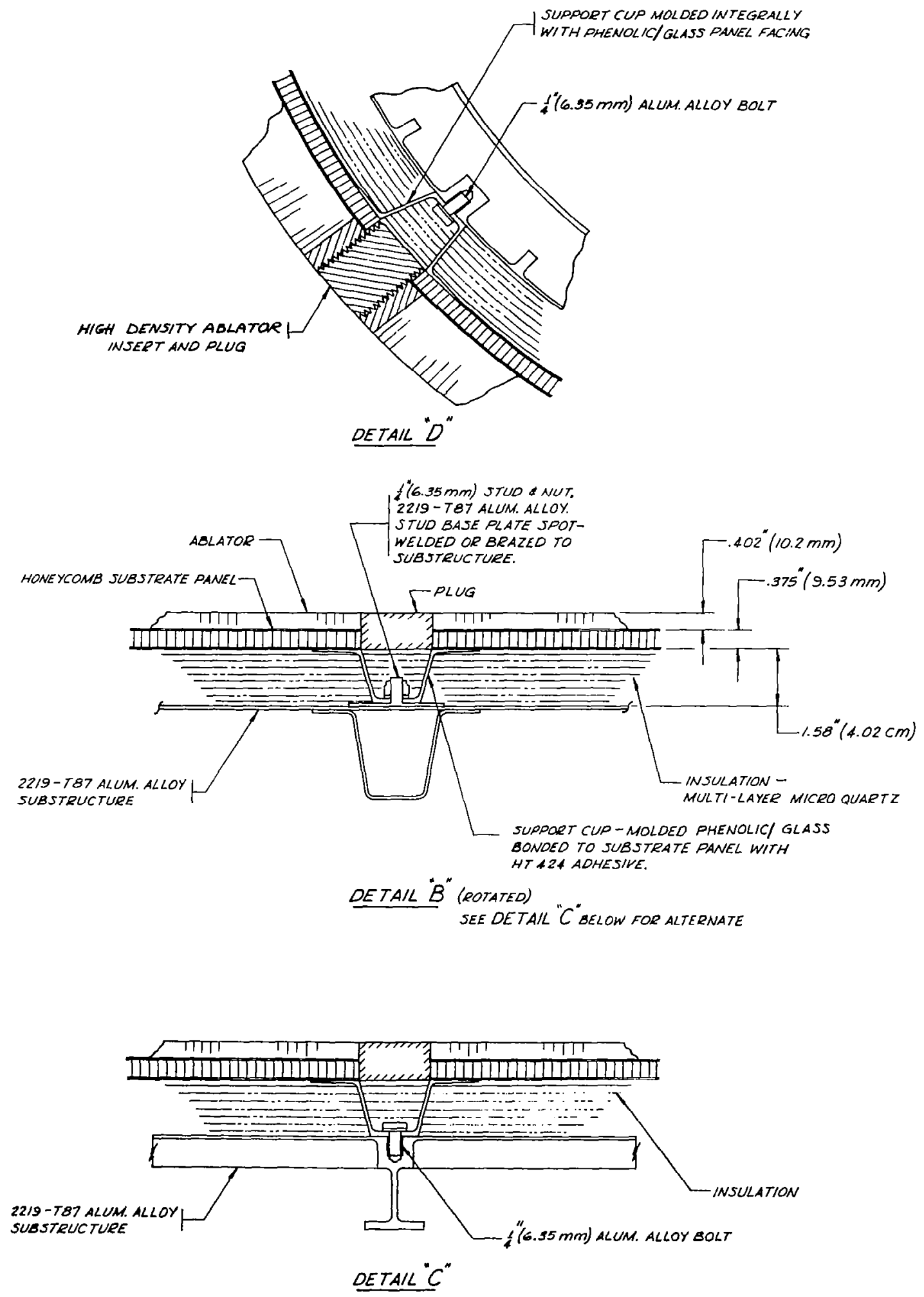

FIGURE 119. ALTERNATE DESIGNS FOR ATTACHMENT 


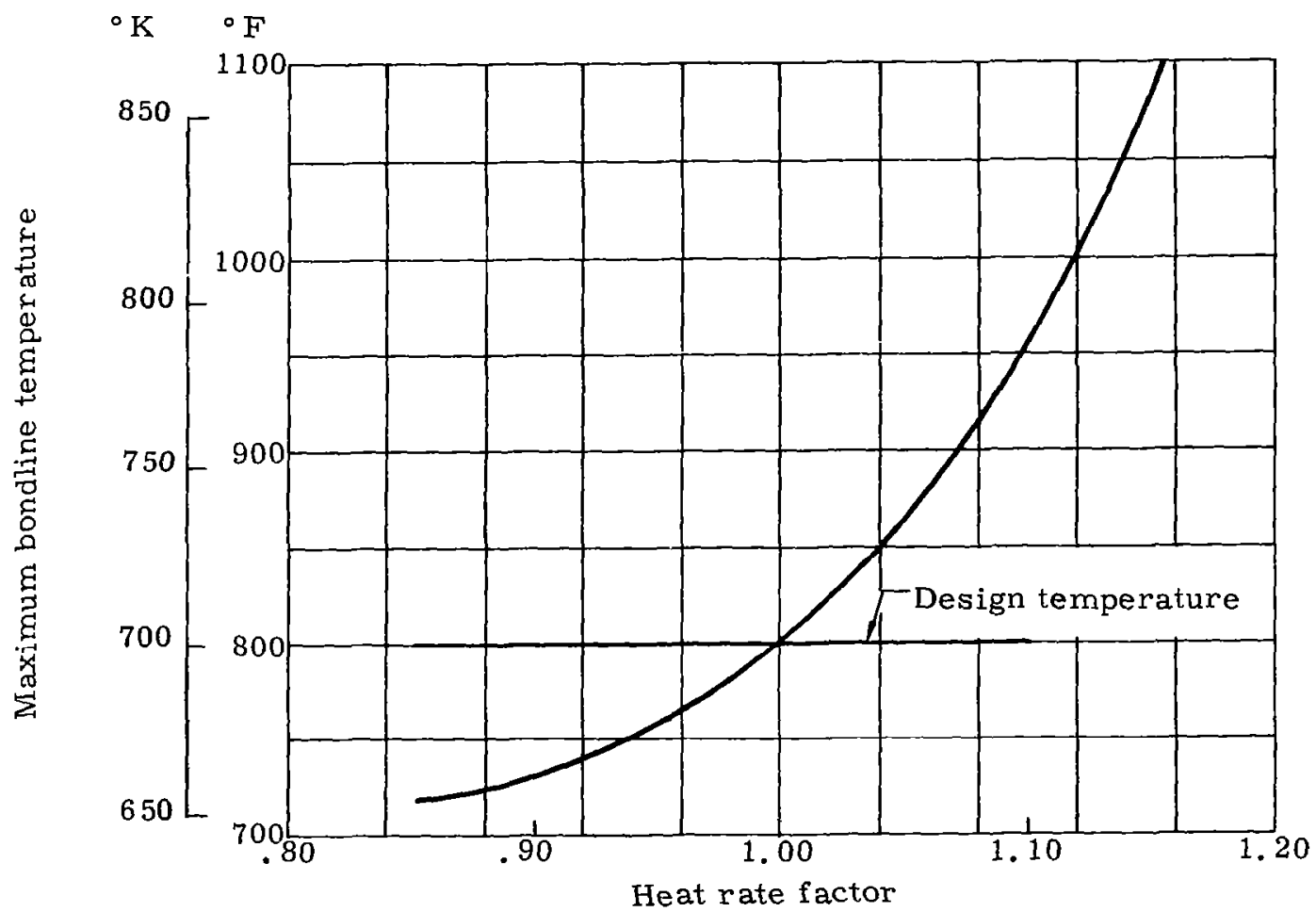

FIGURE 120a. EFFECT OF VARIATION IN HEAT RATE FACTOR ON HEAT SHIELD TEMPERATURES (LDNP ABLATOR, OVERSHOOT RE-ENTRY, LEADING EDGE PANEL,

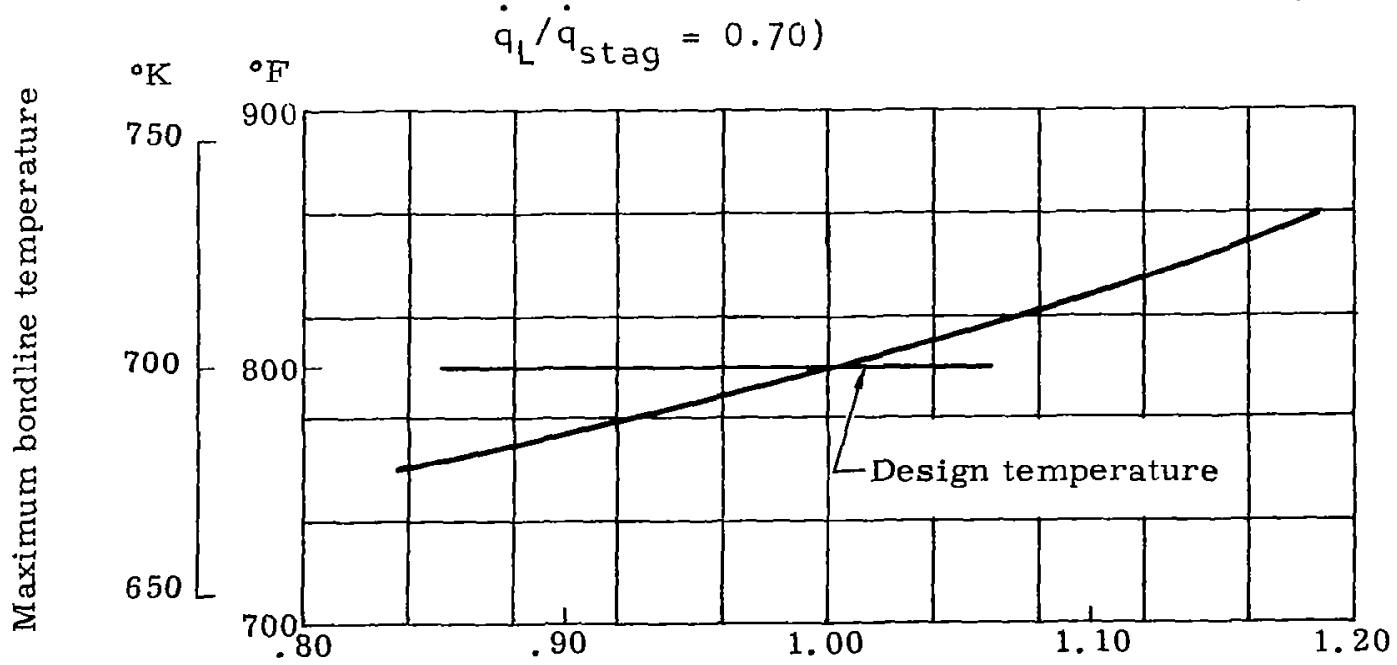

Heat rate factor

FIGURE 120b. EFFECT OF VARIATION IN HEAT RATE FACTOR ON HEAT SHIELD TEMPERATURES (LDNP ABLATOR, OVERSHOOT RE-ENTRY, CROWN PANEL, $\dot{q}_{L} / \dot{q}_{\text {stag }}=0.04$ ) 


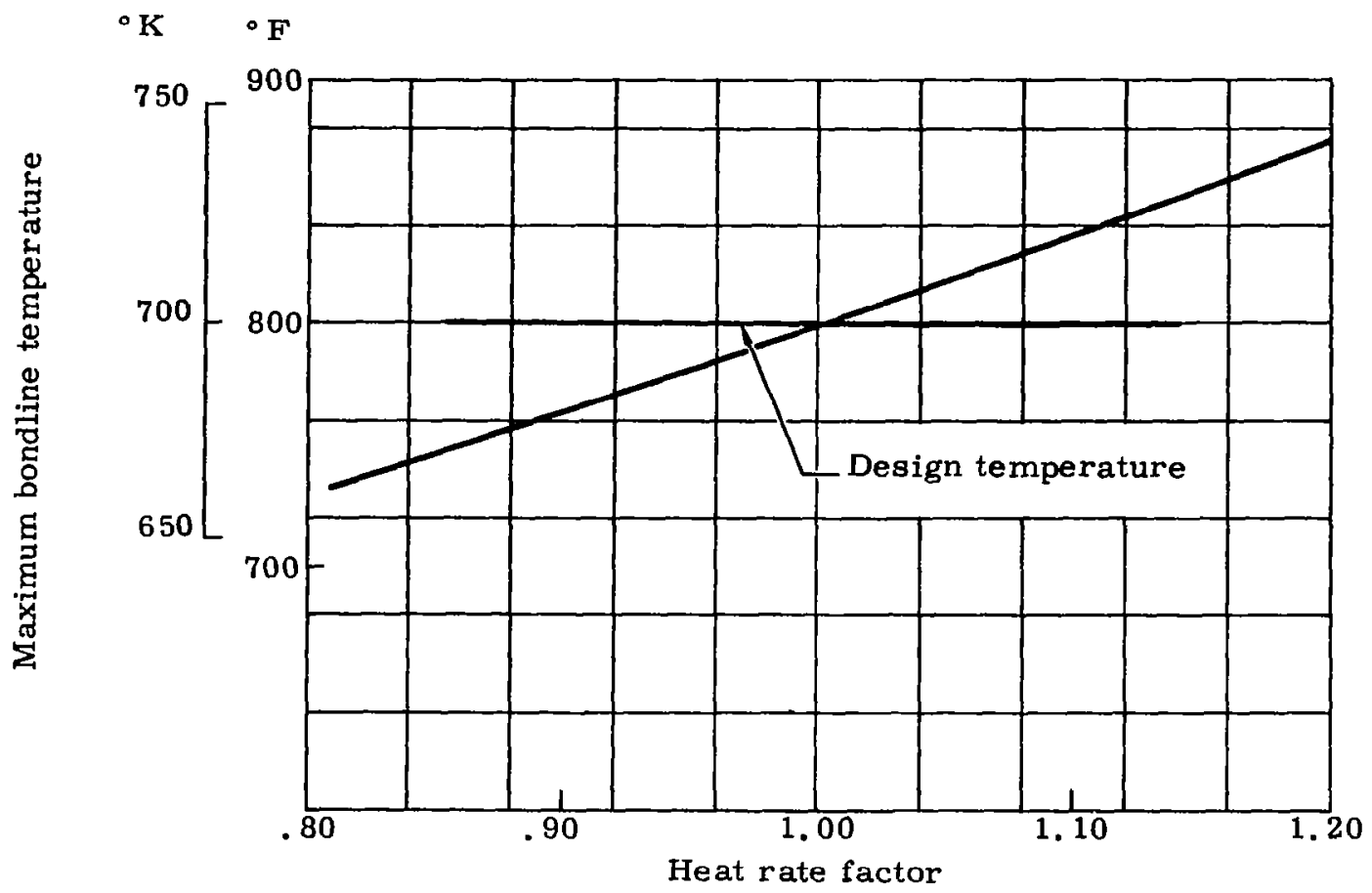

FIGURE 121a. EFFECT OF VARIATION IN HEAT RATE FACTOR ON HEAT SHIELD TEMPERATURES (NASA 602 ABLATOR, OVERSHOOT RE-ENTRY, LEADING EDGE PANEL,

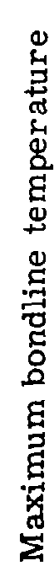

$\left.{ }^{\circ} \mathrm{K} \quad{ }^{\circ} \mathrm{F} \quad \dot{\mathrm{q}}_{\mathrm{L}} / \dot{\mathrm{q}}_{\text {Stag }}=0.70\right)$

${ }_{650}^{750}$

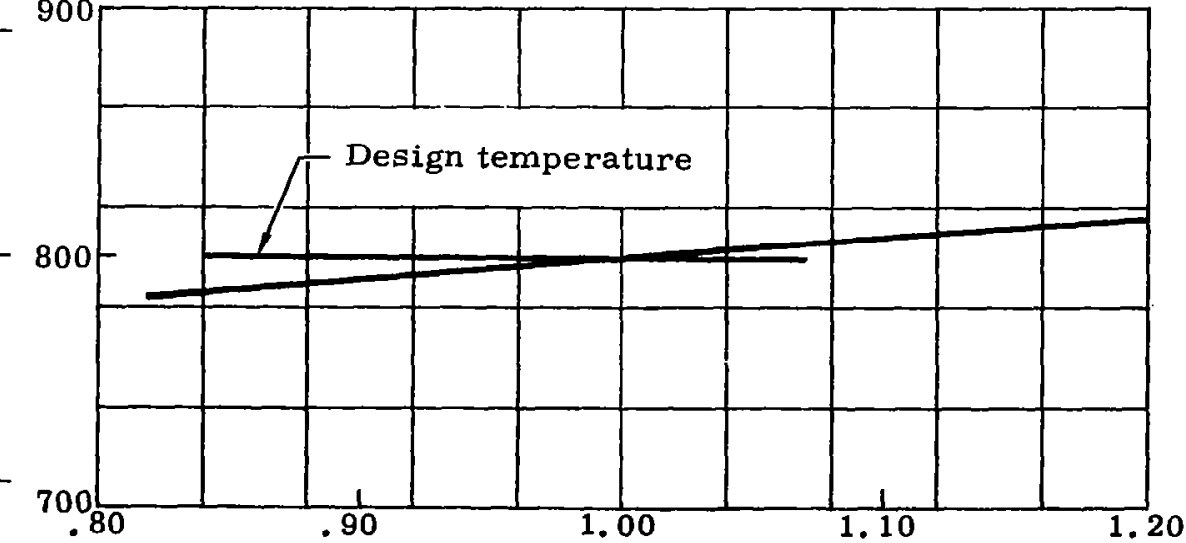

Heat $r$ ate factor

FIGURE 12lb. EFFECT OF VARIATION IN HEAT RATE FACTOR ON HEAT SHIELD TEMPERATURES (NASA 602 ABLATOR, OVERSHOOT RE-ENTRY, CROWN PANEL,

$\dot{q}_{L} / \dot{q}_{\text {stag }}=0.04$ ) 


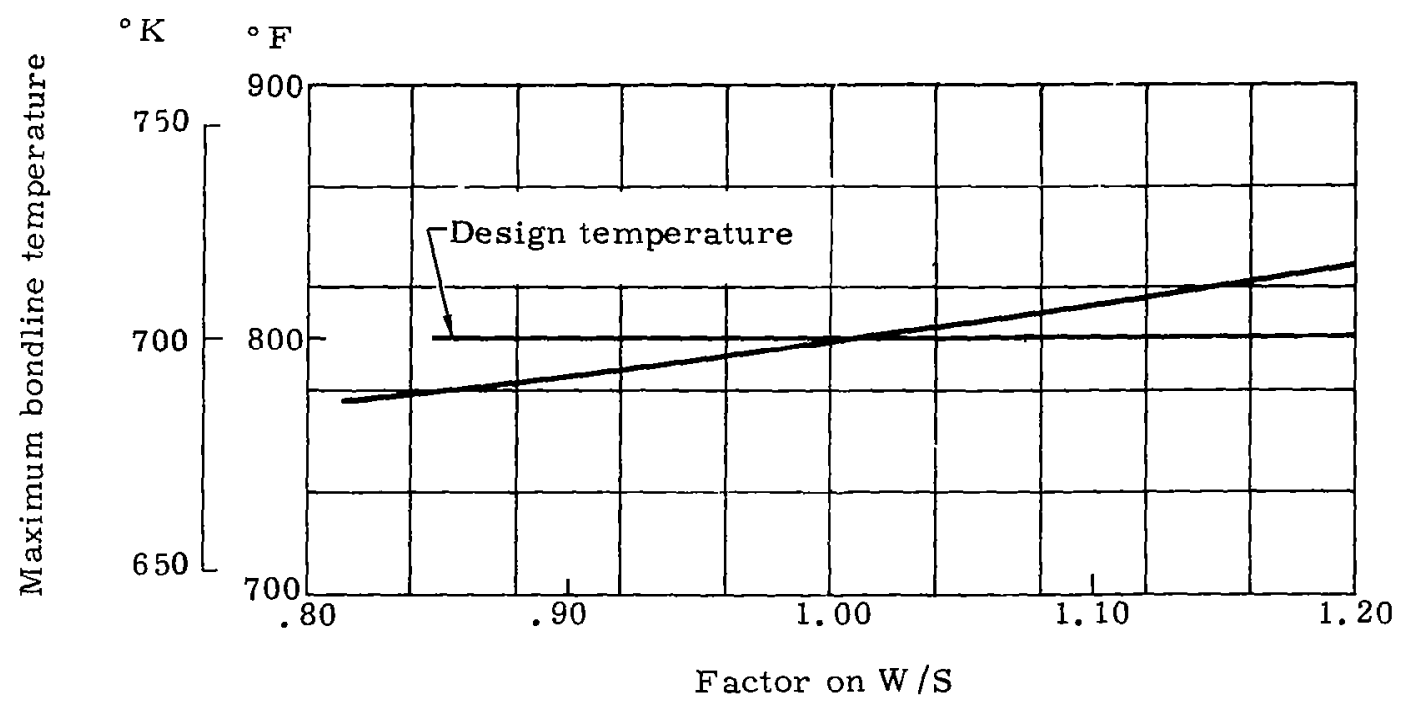

FIGURE 122a. EFFECT OF VARIATION IN VEHICLE W/S ON HEAT SHIELD TEMPERATURES (NASA 602 ABLATOR, NOMINAL RE-ENTRY, LEADING EDGE PANEL,

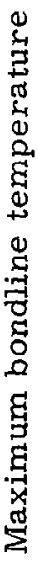

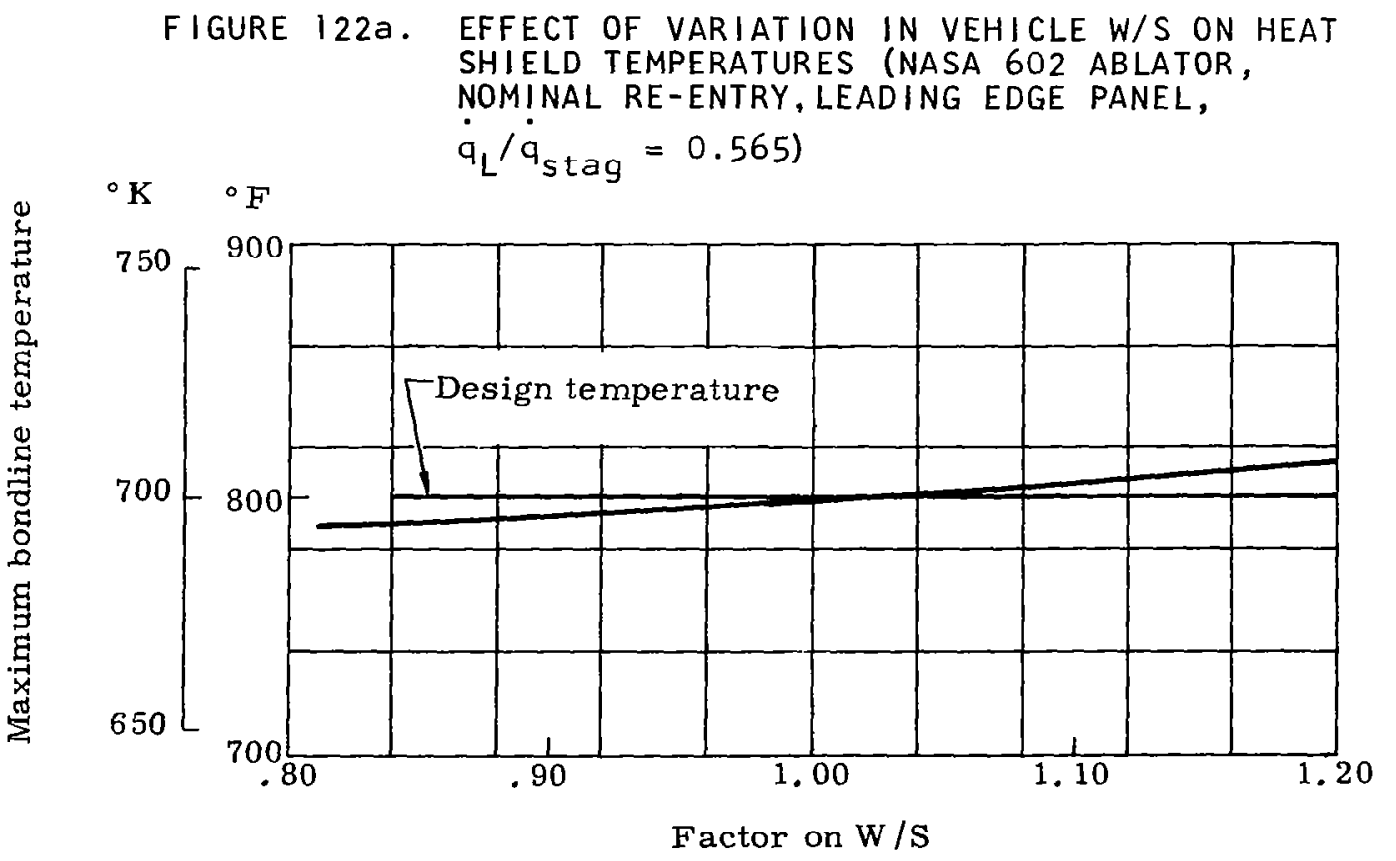

FIGURE 122b. EFFECT OF VARIATION IN VEHICLE W/S ON HEAT SHIELD TEMPERATURES (NASA 602 ABLATOR, NOMINAL RE-ENTRY, CROWN PANEL, $\dot{q}_{L} / \dot{q}_{\text {stag }}=0.05$ ) 


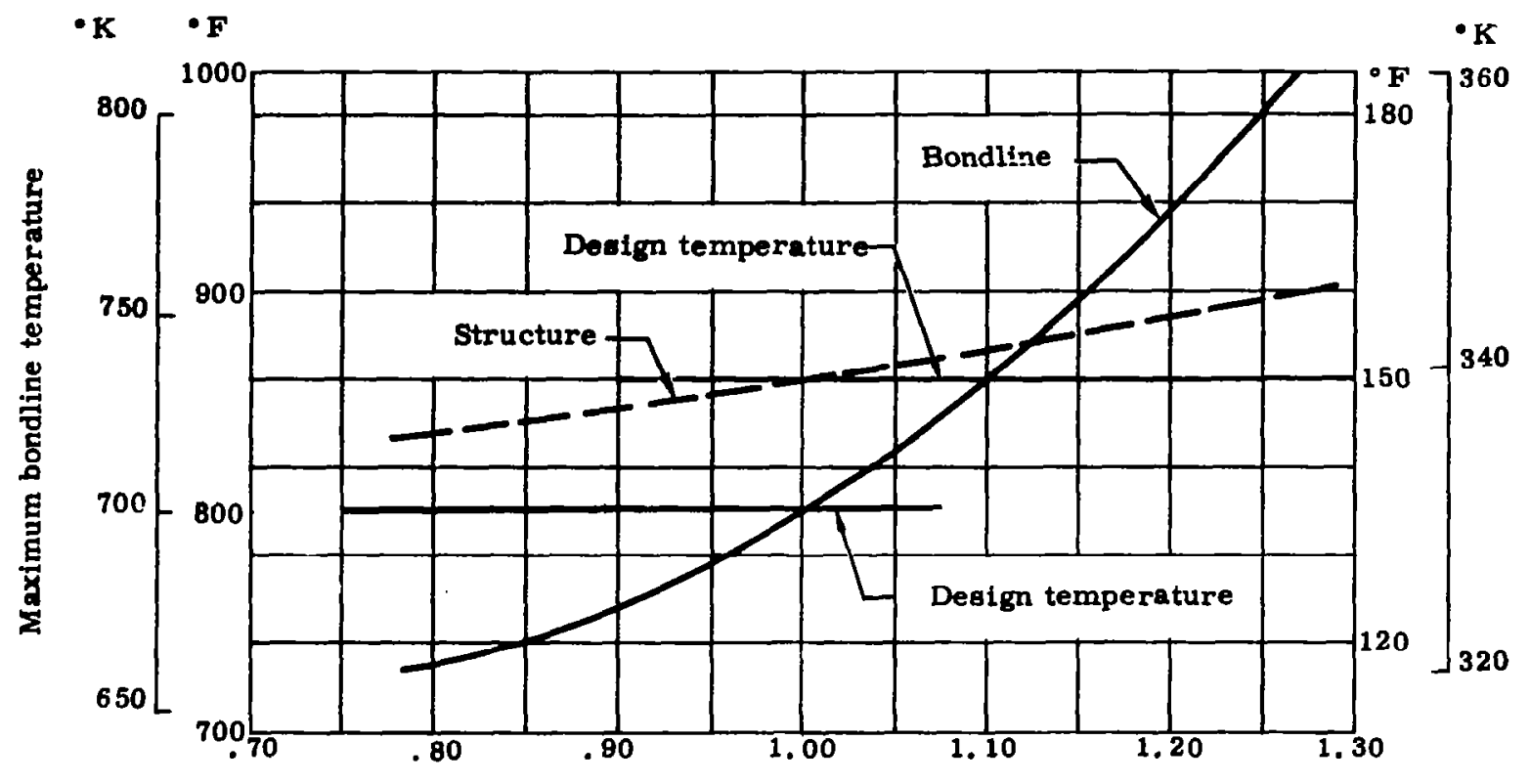

Ablator thermal conductivity factor

FIGURE 123a. EFFECT OF VARIATION IN ABLATOR THERMAL CONDUCTIVITY ON HEAT SHIELD TEMPERATURES (LDNP ABLATOR, OVERSHOOT

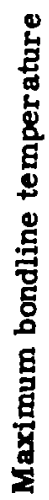
RE-ENTRY, LEADING EDGE PANEL, $\dot{q}_{L} / \dot{q}_{\text {stag }}=0.70$ )

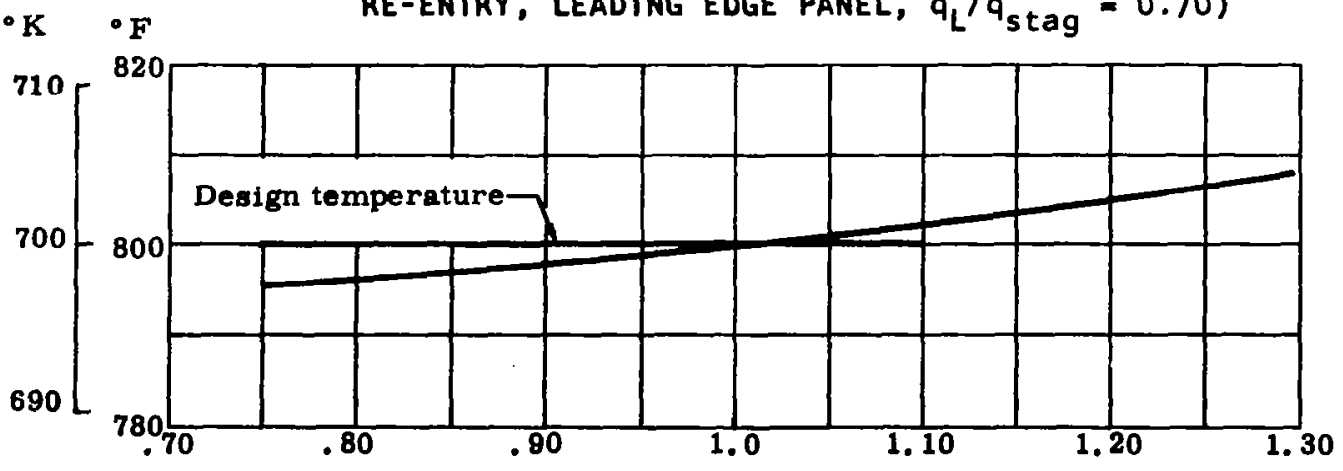

Ablator thermal conductivity factor

FIGURE 123b. EFFECT OF VARIATION IN ABLATOR THERMAL CONDUCTIVITY ON HEAT SHIELD TEMPERATURES (LONP ABLATOR, OVERSHOOT

RE-ENTRY, CROWN PANEL, $\dot{q}_{L} / \dot{q}_{\text {stag }}=0.04$ ) 


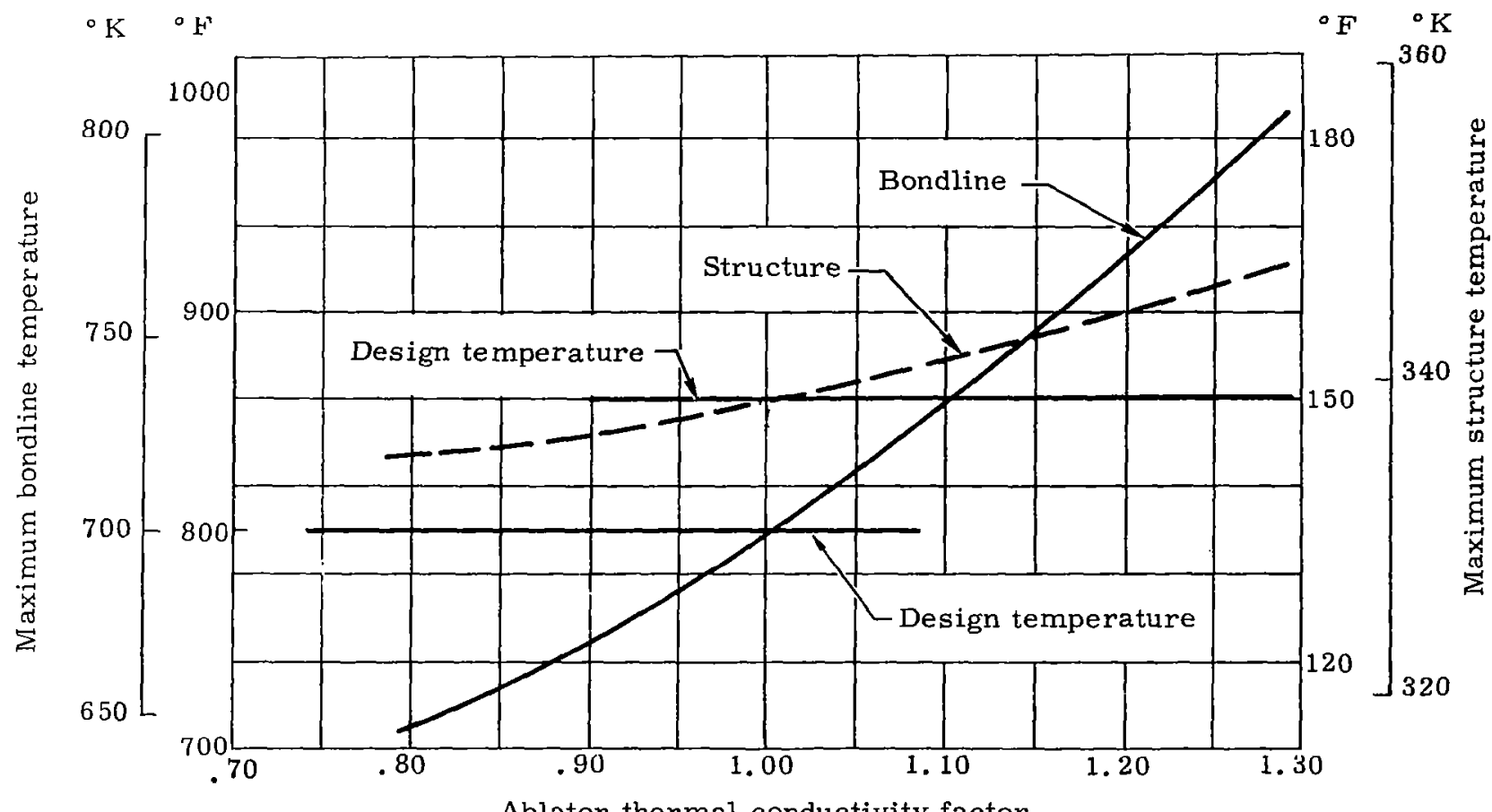

Ablator thermal conductivity factor

FIGURE 124a. EFFECT OF VARIATION IN ABLATOR THERMAL CONDUCTIVITY ON HEAT SHIELD TEMPERATURES (NASA 602 ABLATOR, OVERSHOOT

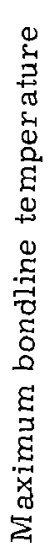

${ }^{\circ} \mathrm{K}{ }^{\circ} \mathrm{F} \quad \mathrm{RE}-$ ENTRY, LEADING EDGE PANEL, $\dot{q}_{\mathrm{L}} / \dot{q}_{\mathrm{stag}}=0.70$ )

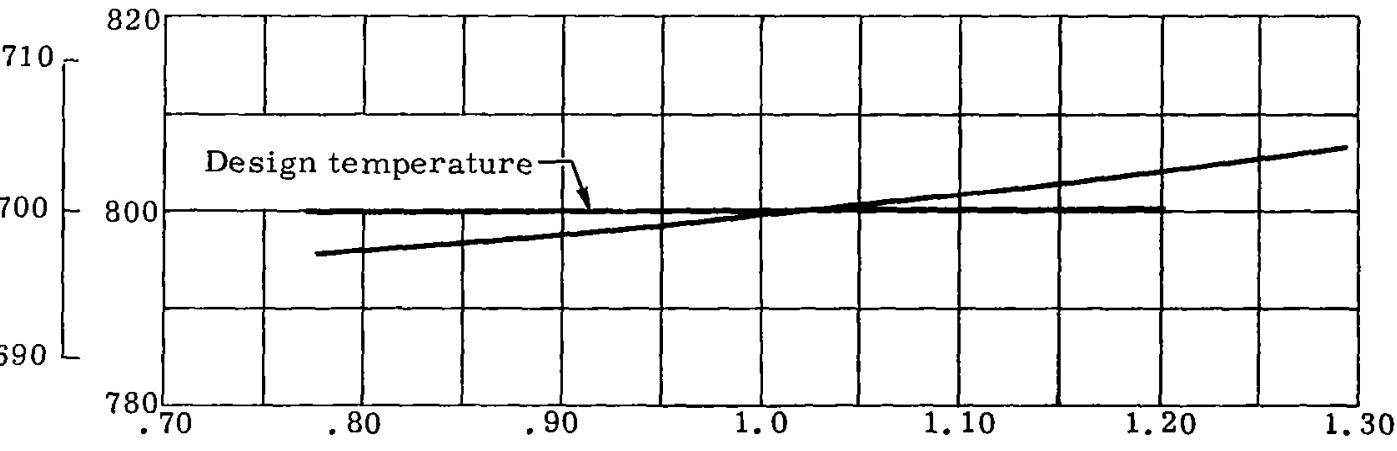

Ablator thermal conductivity factor

FIGURE 124b. EFFECT OF VARIATION IN ABLATOR THERMAL CONDUCTIVITY ON HEAT SHIELD TEMPERATURES (NASA 602 ABLATOR, OVERSHOOT RE-ENTRY, CROWN PANEL, $\dot{\mathrm{q}}_{\mathrm{L}} / \dot{\mathrm{q}}_{\text {stag }}=0.04$ ) 


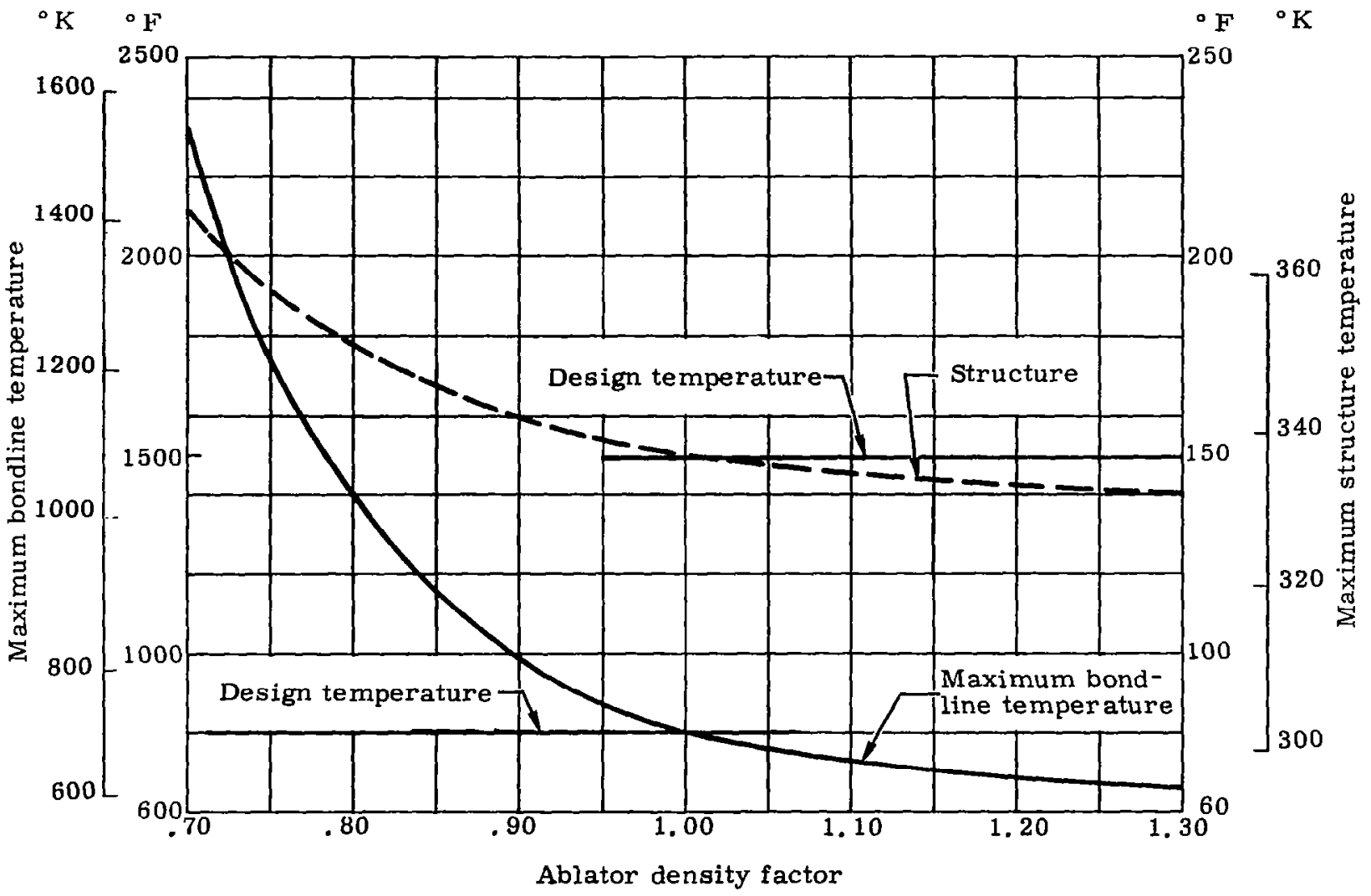

FIGURE 125a. EFFECT OF VARIATION IN ABLATOR DENSITY ON HEAT SHIELD TEMPERATURES (LDNP ABLATOR, OVERSHOOT RE - ENTRY, LEADING EDGE PANEL, $\left.\dot{q}_{L} / \dot{q}_{s t a g}=0.70\right)$

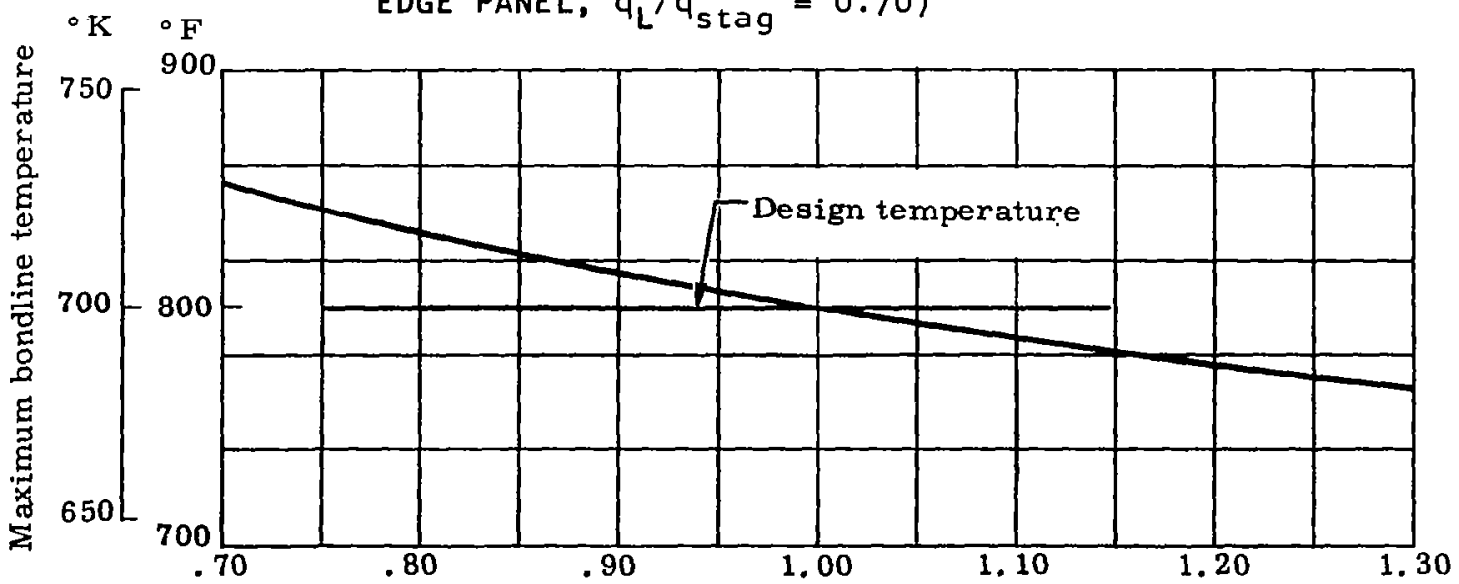

Ablator density factor

FIGURE 125b. EFFECT OF VARIATION IN ABLATOR DENSITY ON HEAT SHIELD TEMPERATURES (LDNP ABLATOR, OVERSHOOT RE-ENTRY, CROWN PANEL, $\dot{q}_{L} / \dot{q}_{\text {stag }}=0.04$ ) 


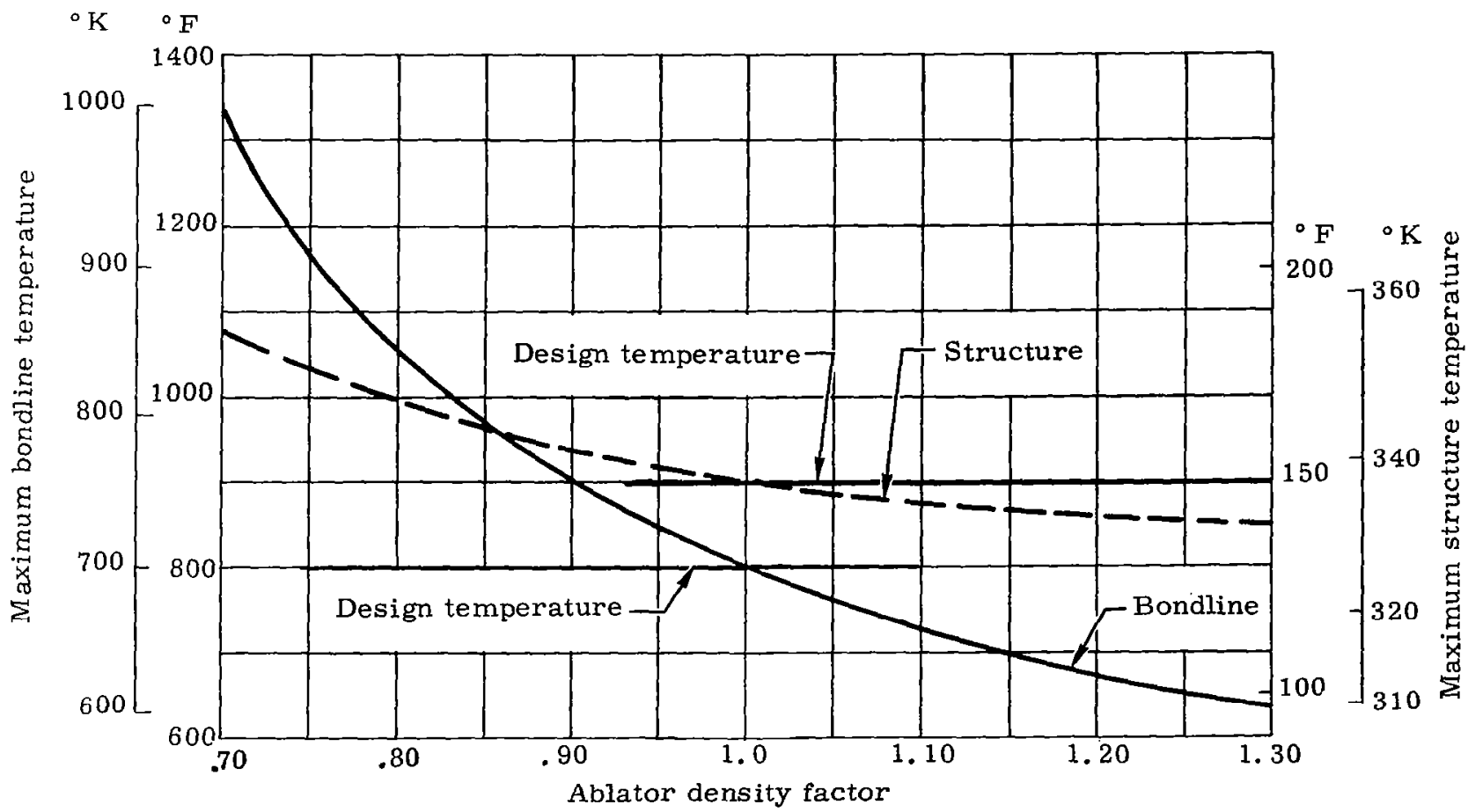

FIGURE 126a. EFFECT OF VARIATION IN ABLATOR DENSITY ON HEAT SHIELD TEMPERATURES (NASA 602 ABLATOR, OVERSHOOT RE-ENTRY, LEADING EDGE PANEL, $\left.\dot{q}_{L} / \dot{q}_{\text {Stag }}=0.70\right)$

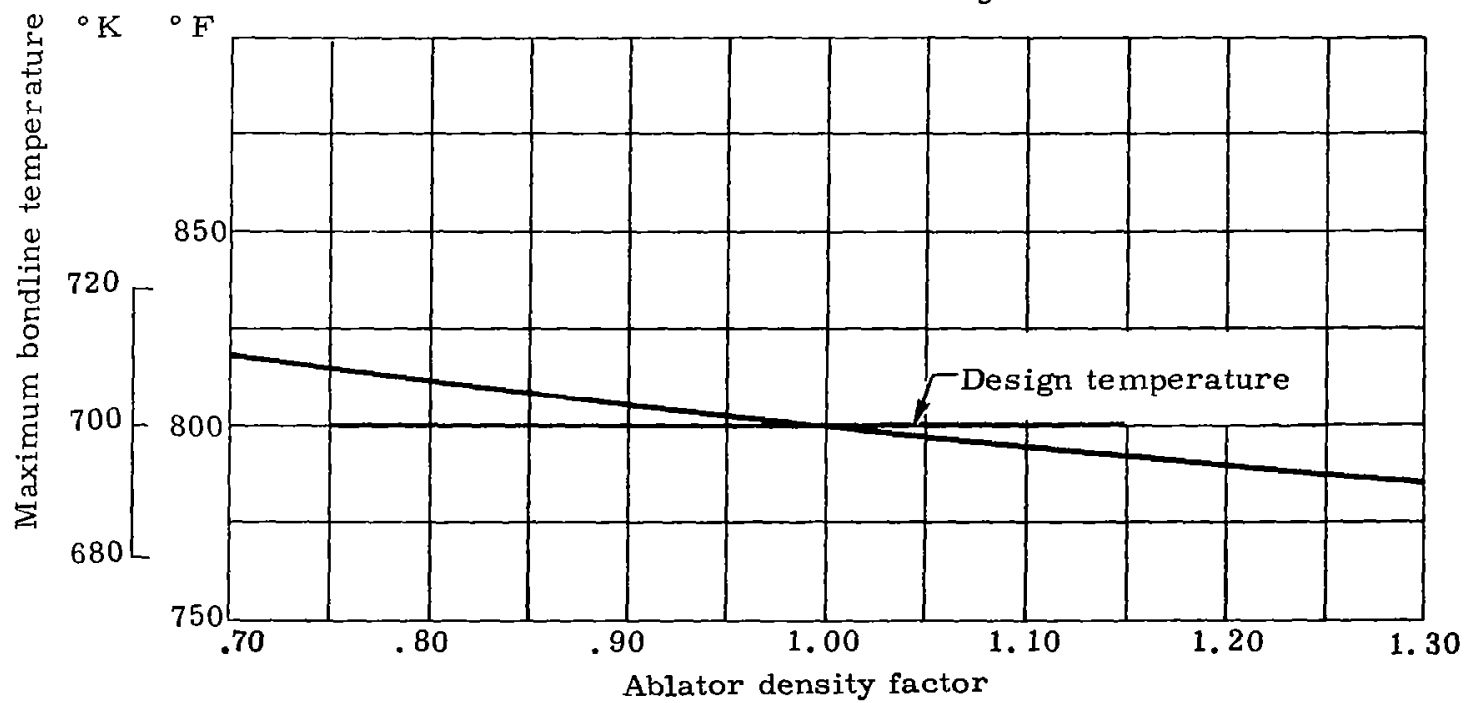

FIGURE 126b. EFFECT OF VARIATION IN ABLATOR DENSITY ON HEAT SHIELD TEMPERATURES (NASA 602 ABLATOR, OVERSHOOT RE-ENTRY, CROWN PANEL, $\dot{q}_{L} / \dot{q}_{\text {stag }}=0.04$ ) 


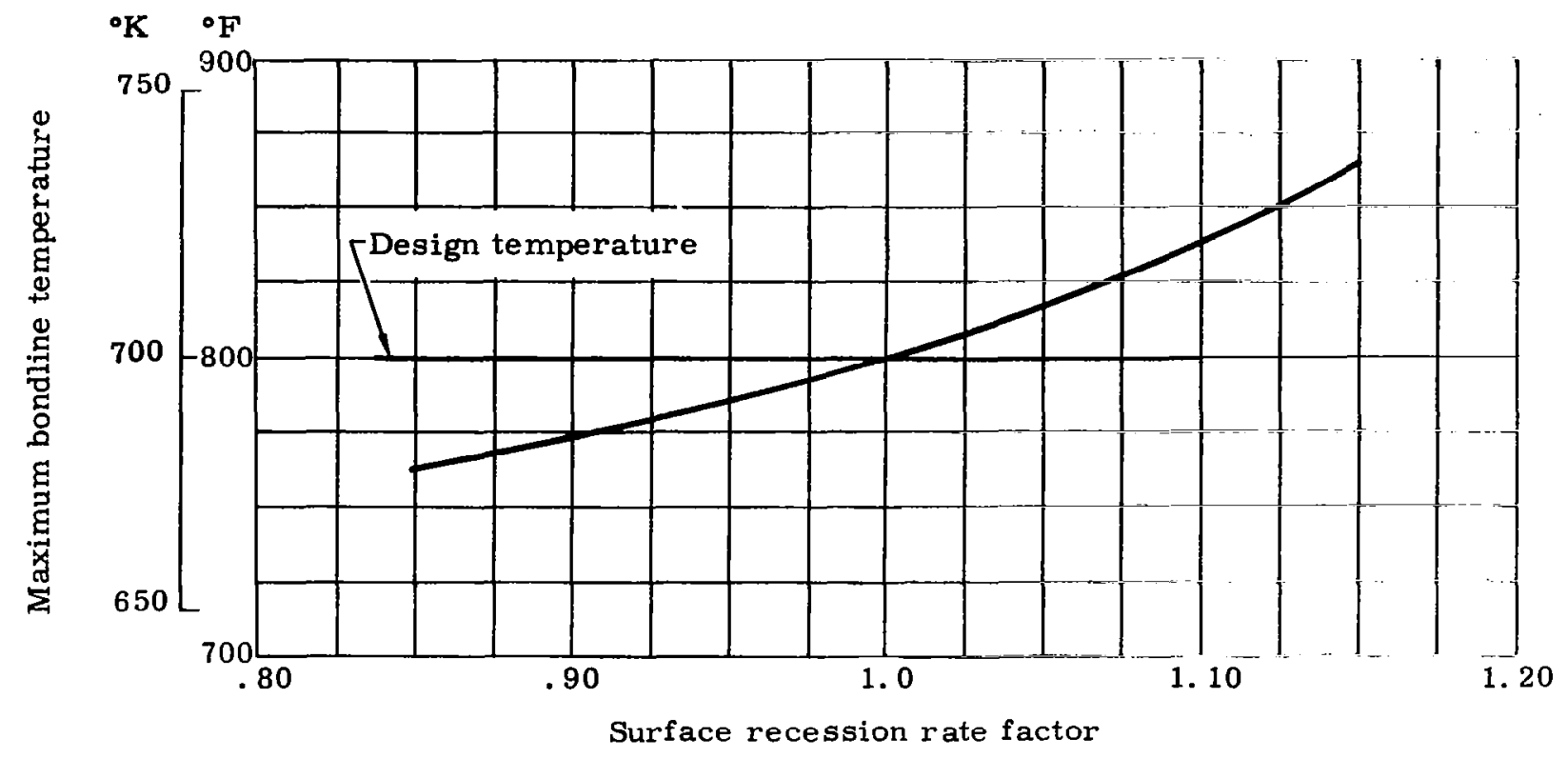

FIGURE 127a. EFFECT OF VARIATION IN CHAR SURFACE RECESSION RATES ON HEAT SHIELD TEMPERATURES (LDNP ABLATOR, OVERSHOOT RE-ENTRY, LEADING EDGE PANEL, $\dot{q}_{L} / \dot{q}_{\text {stag }}=0.70$ )

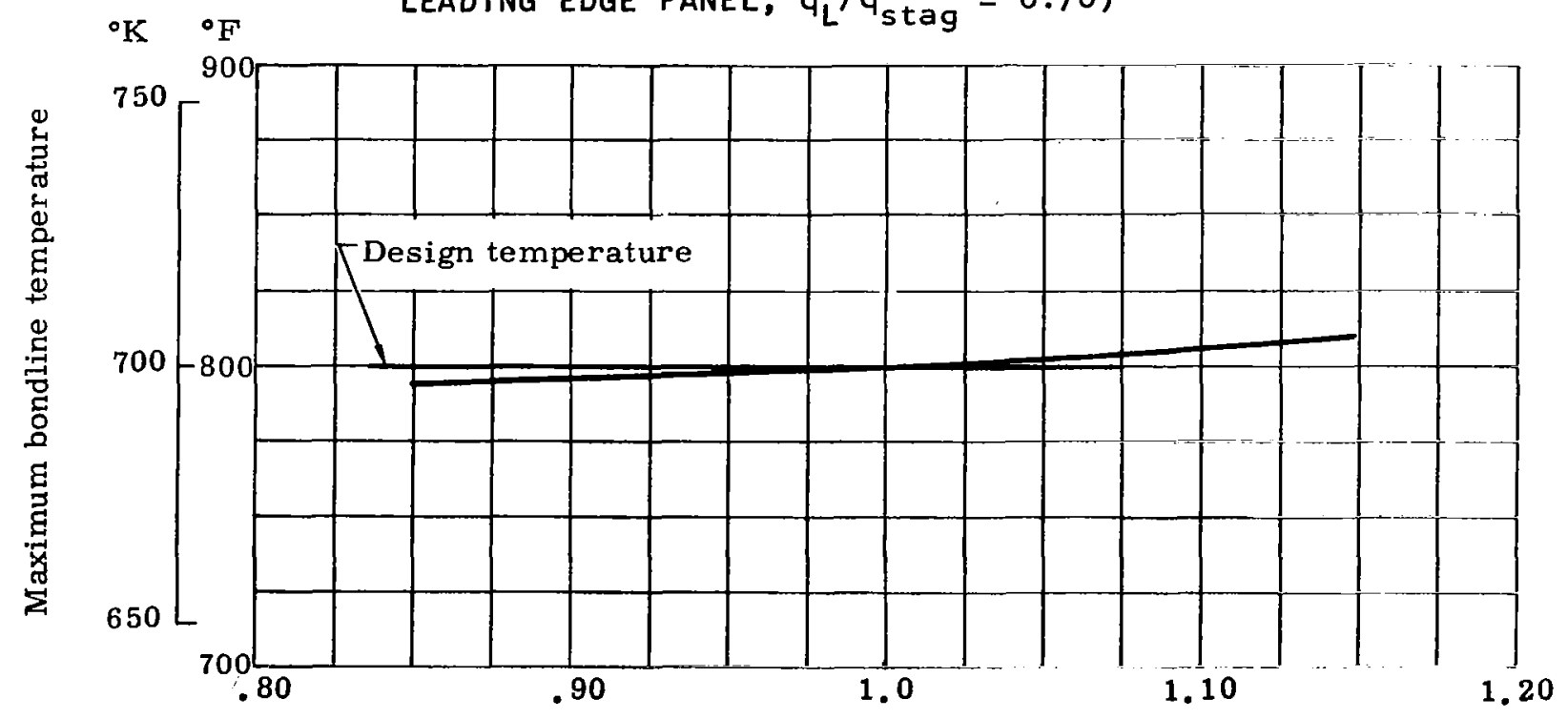

Surface recession rate factor

FIGURE 127b. EFFECT OF VARIATION IN CHAR SURFACE RECESSION RATES ON HEAT SHIELD TEMPERATURES (LDNP ABLATOR, OVERSHOOT RE-ENTRY, $\dot{\mathrm{q}}_{\mathrm{L}} / \dot{\mathrm{q}}_{\text {stag }}=0.20$ ) 


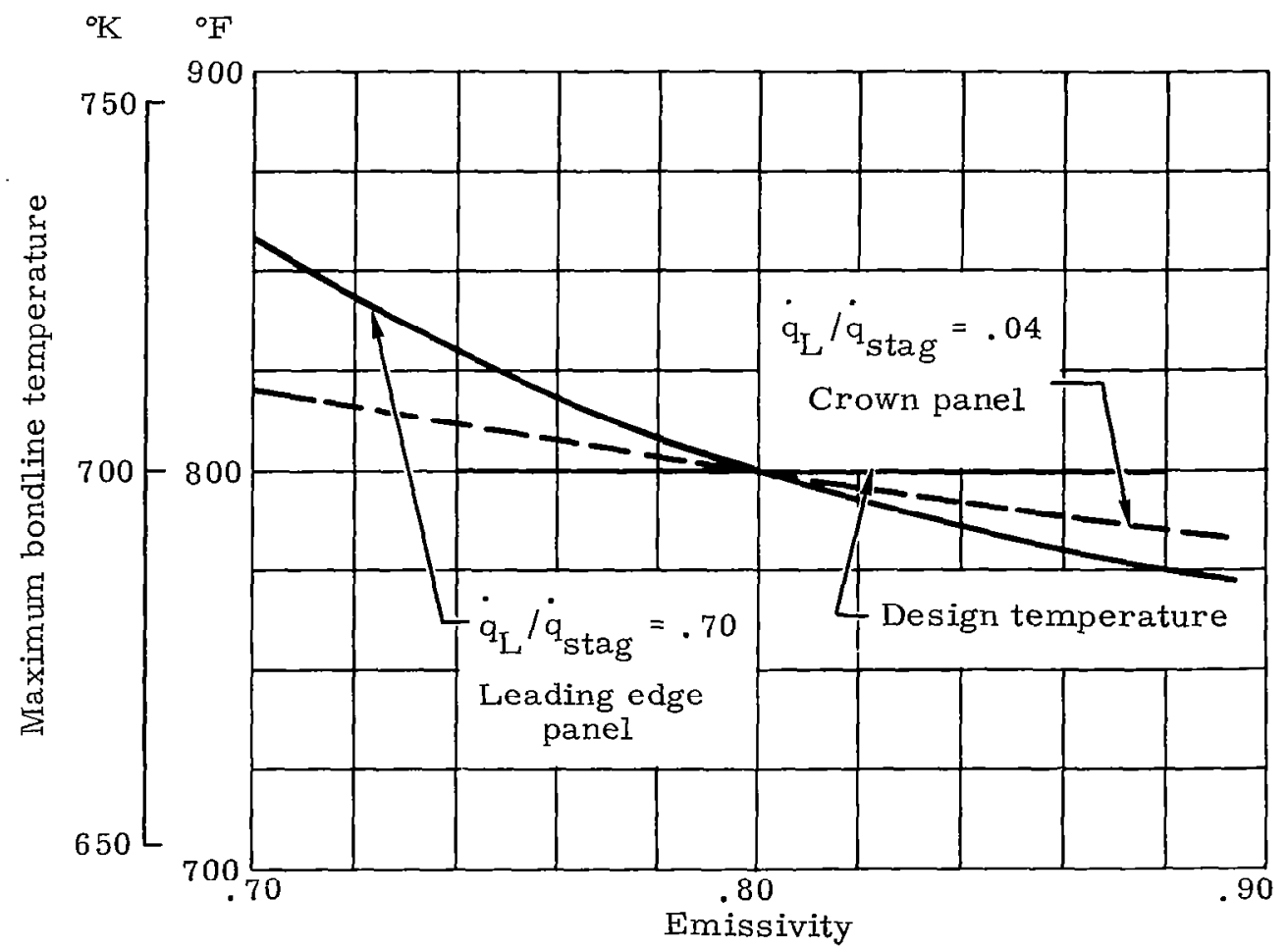

FIGURE 128a. EFFECT OF VARIATION OF CHAR EMISSIVITY ON HEAT SHIELD TEMPERATURES (LENP ABLATOR, OVERSHOOT RE-ENTRY)

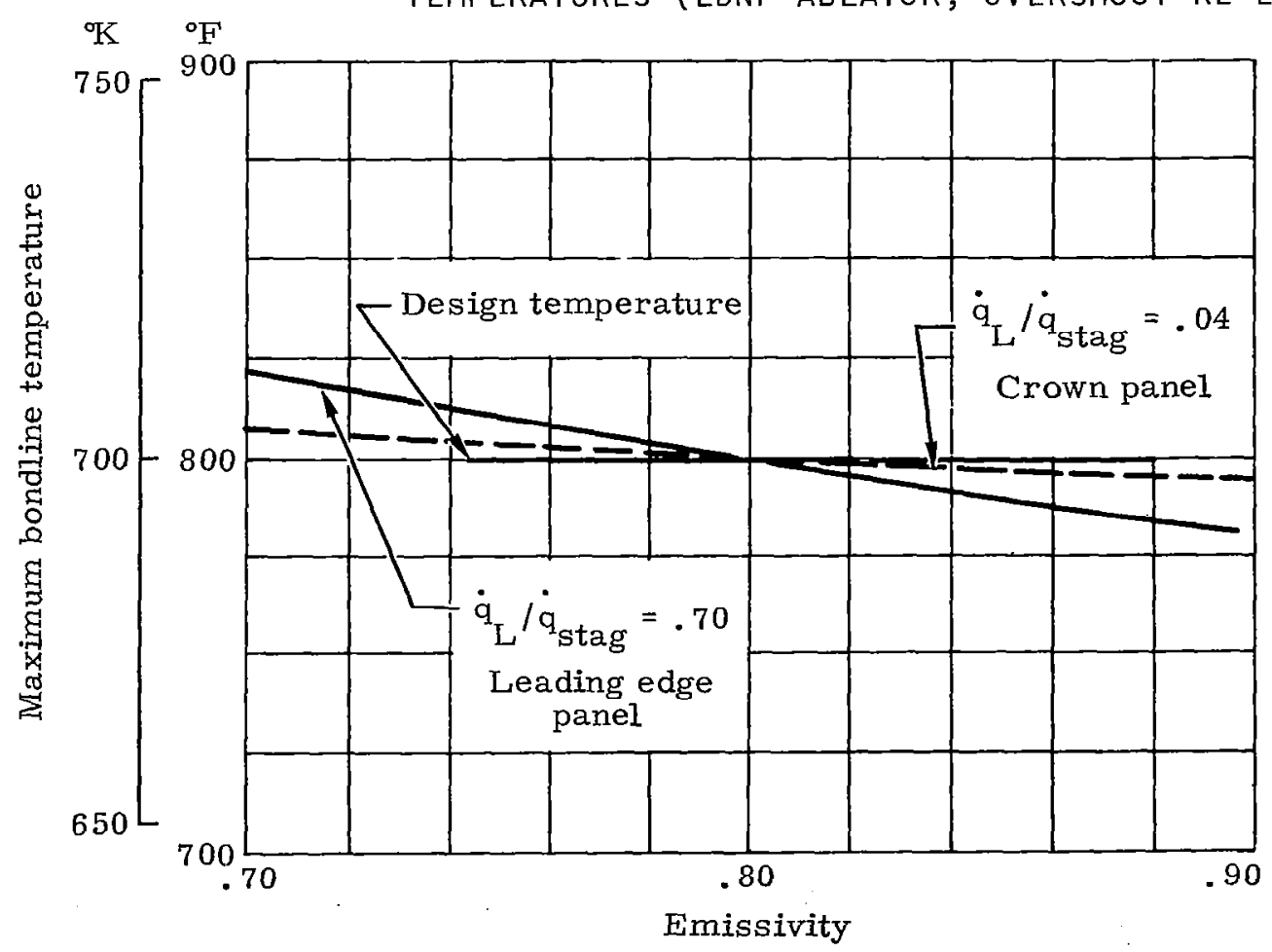

FIGURE 128b. EFFECT OF VARIATION OF CHAR EMISSIVITY ON HEAT SHIELD TEMPERATURES (NASA 602 ABLATOR, OVERSHOOT. RE-ENTRY) 


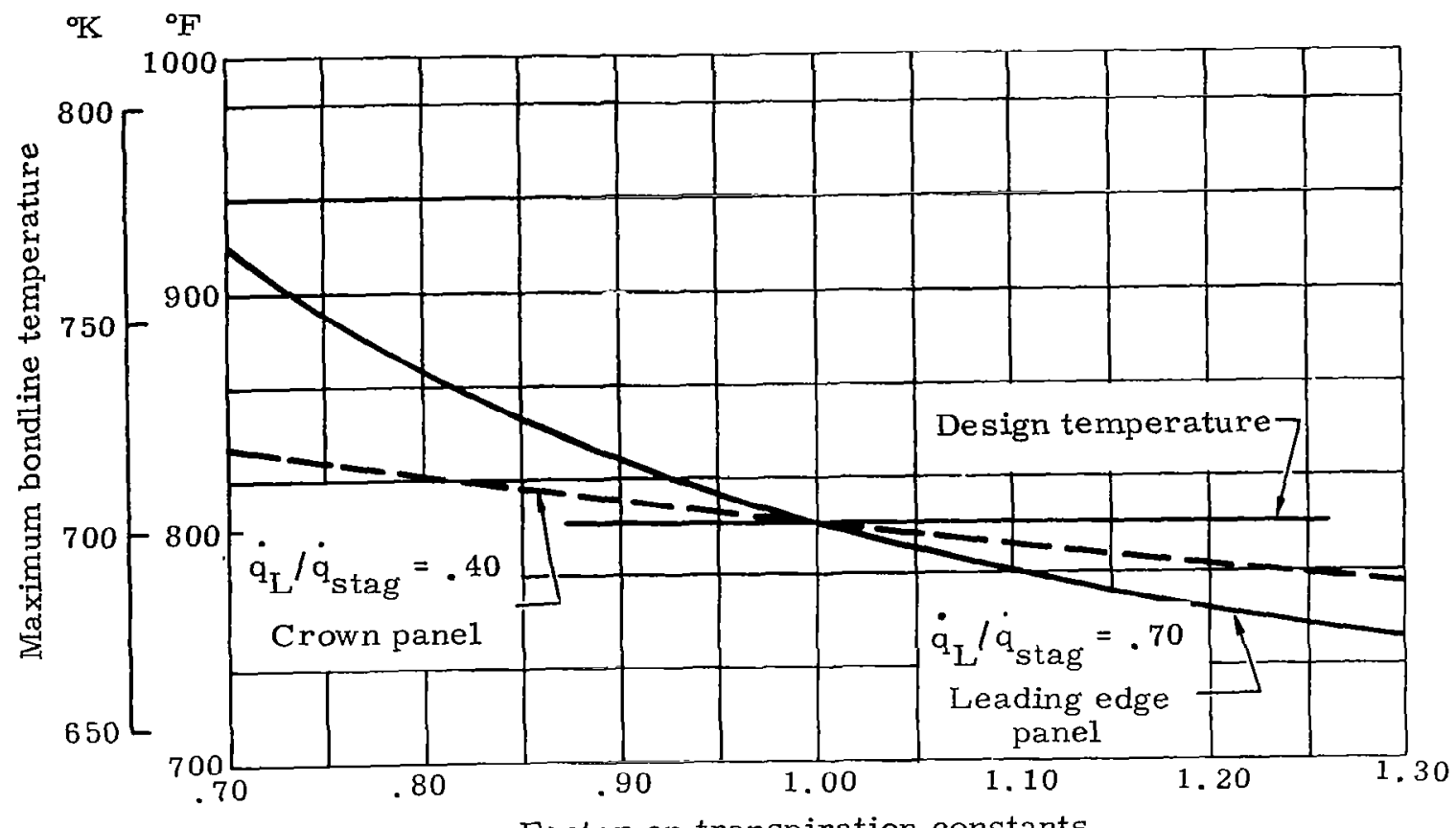

Factor on transpiration constants

FIGURE 129a. EFFECT OF VARIATION OF TRANSPIRATION FACTOR ON HEAT
SHIELD TEMPERATURES (LDNP ABLATOR, OVERSHOOT RE-ENTRY)

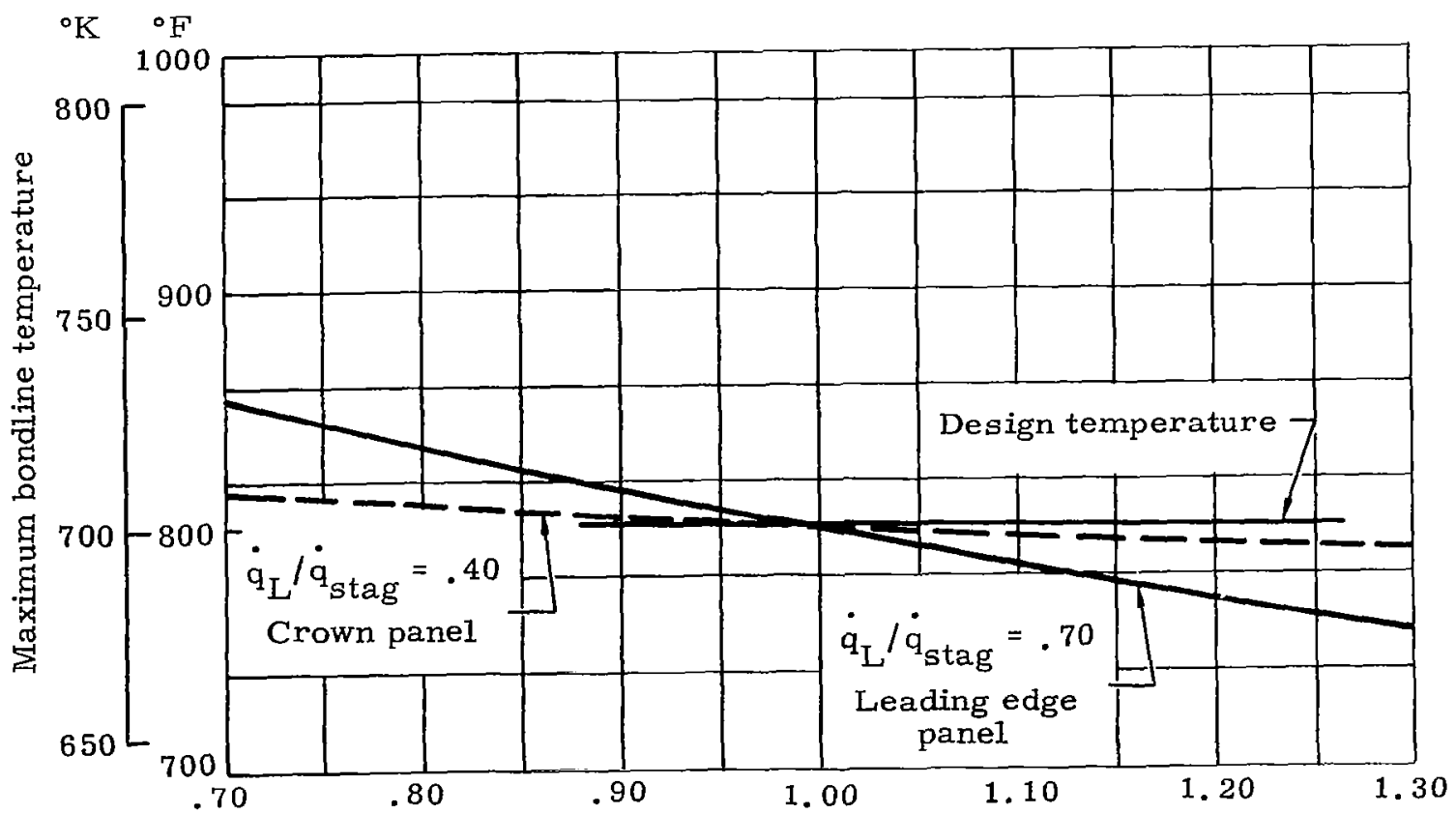

Factor on transpiration constants.

FIGURE 129b. EFFECT OF VARIATION OF TRANSPIRATION FACTOR ON HEAT SHIELD TEMPERATURES (NASA 602 ABLATOR, OVERSHOOT RE-ENTRY) 